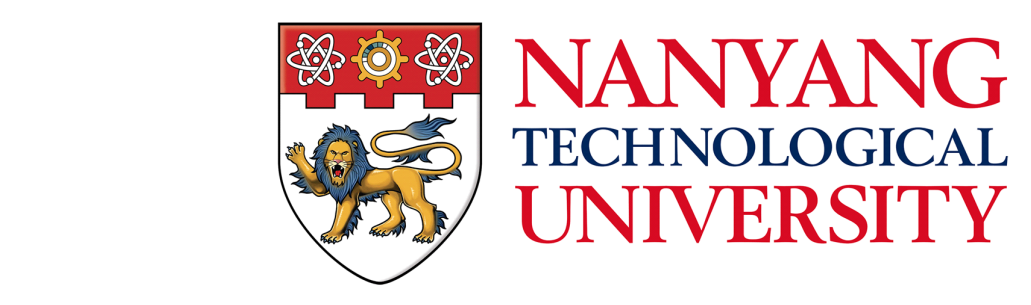

\title{
Computability Theory and Algebra
}

\author{
Wu Huishan
}

School of Physical and Mathematical Sciences

A thesis submitted to the Nanyang Technological University in partial fulfilment of the requirement for the degree of Doctor of Philosophy 



\section{Acknowledgments}

I would like to thank my supervisor, Prof. Wu Guohua, for teaching me computability theory and guiding my research in the past few years. His knowledge, suggestions, and discussions inspire me a lot, and I appreciate him for sharing me with his ideas. His style of teaching, writing of papers and presentations influence me a lot. I am grateful to him for all his efforts for guiding me to be a teacher and to be a researcher. I also want to thank him for supporting my PhD study by his research fund from MOE of Singapore.

I would like to thank Prof. Ng Keng Meng, for teaching me a course on firstorder logic. I also want to thank my peers and friends, Wang Shaoyi, Ru Junren, Yu Hongyuan, Yuan Bowen for many inspiring discussion and various seminars on logic and computability theory.

Last, but not the least, I want to thank my parents for their love and support. 


\section{Contents}

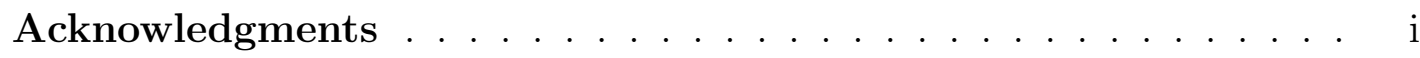

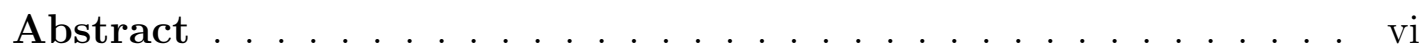

1 Introduction 1

1.1 Basics of computability theory . . . . . . . . . . . . . . . 1

1.2 Part I: Bounded-jump operator and the high/low hierarchy . . . . . 8

1.3 Part II: Degrees of orders on torsion-free abelian groups . . . . . . . . 11

1.4 Part III: Reverse mathematics, abelian groups and modules . . . . . . 17

\section{Bounded-jump operator and the high/low hierarchy 26}

2 A bounded-low c.e. set which is low, but not superlow 27

2.1 Requirements and strategies . . . . . . . . . . . . 28

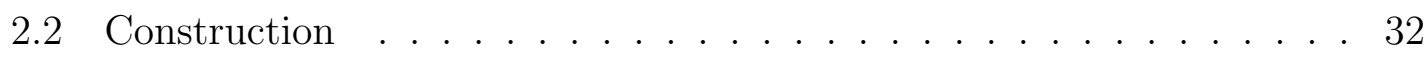

2.3 Verification . . . . . . . . . . . . . . . . 33

2.4 A bounded-low set with Turing degree $\mathbf{0}^{\prime} \ldots \ldots \ldots \ldots$

3 A bounded-high set which is high, but not superhigh 37

3.1 Pseudo-jump inversion via bounded-high sets . . . . . . . . . . 38

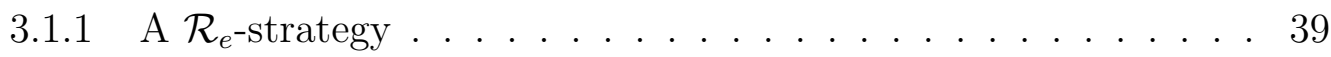

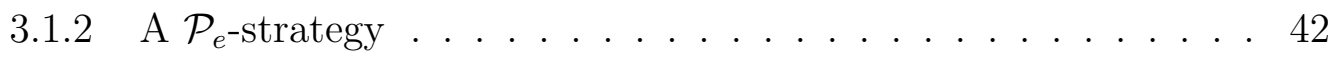

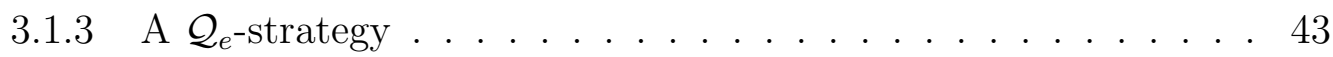

3.1.4 Define the computable function $g_{r} \ldots \ldots \ldots \ldots \ldots$

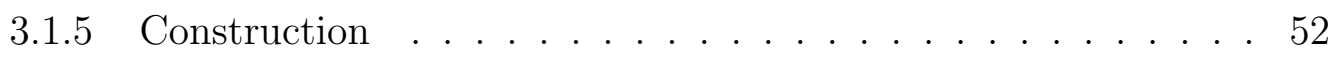

3.1 .6 Verification . . . . . . . . . . . . . 57

3.2 Pseudo-jump inversion via bounded-low c.e. sets . . . . . . . . . 60 
4 Group-order-computable degrees and PA degrees $\quad 64$

4.1 Requirements . . . . . . . . . . . . . . . . . 65

4.2 Background . . . . . . . . . . . . . . . . . 66

4.3 The $\mathcal{P}$-strategy $\ldots \ldots \ldots \ldots$. . . . . . . . . . . . . . . 67

4.4 The $\mathcal{Q}$-strategy . . . . . . . . . . . . . . . . . . 70

4.5 The $\mathcal{R}$-strategy . . . . . . . . . . . . . . . . . . . . . 73

4.5.1 Background on PA-complete sets . . . . . . . . . 73

4.5.2 The $\mathcal{R}^{\prime}$-strategy . . . . . . . . . . . . . . . . . . 75

4.6 Construction and verification . . . . . . . . . . . . 82

4.6 .1 Construction . . . . . . . . . . . . . 82

4.6.2 $\mathscr{G}$ is a computable group possessing computable orders . . . . 82

4.6.3 A-computable orders on $\mathscr{G}$ which are not computable . . . . . 84

4.6.4 Orders on $\mathscr{G}$ which are computable from PA-complete sets . . 90

5 Group orders and c.e. degrees $\quad 91$

5.1 Requirements and strategies . . . . . . . . . . . . . . . . 91

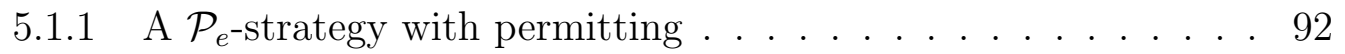

5.1 .2 The $\mathcal{Q}_{0}$-strategy . . . . . . . . . . . . . 93

5.1 .3 The $\mathcal{Q}_{1}$-strategy . . . . . . . . . . . . . . 97

5.1.4 The $\mathcal{Q}$-strategy . . . . . . . . . . . . . . . . . 99

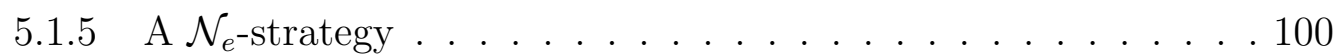

5.2 Construction . . . . . . . . . . . . . . . . . . 107

5.3 Verification . . . . . . . . . . . . . . . . . . 109

III Reverse mathematics, abelian groups and modules 113

6 Reverse mathematics and divisible abelian groups $\quad 114$

6.1 Divisible subgroups . . . . . . . . . . . . . . . . . . . . . . 114

6.2 Two decomposition theorems . . . . . . . . . . . . . . 118 
7 Reverse mathematics and modules

7.1 Projective modules . . . . . . . . . . . . . . . . . . . . . . . . . . . 124

7.1.1 Direct sums of projective modules . . . . . . . . . . . 124

7.1.2 Free modules and projective modules over $\Sigma_{1}^{0}$-PIDs . . . . . 127

7.2 Injective modules . . . . . . . . . . . . . . . . . . 129

7.2.1 Baer's criterion for injective modules . . . . . . . . . . 129

7.2.2 Divisible modules and injective modules over $\Sigma_{1}^{0}$-PIDs . . . . . 132

References

135 


\section{Abstract}

This thesis mainly focuses on classical computability theory and effective aspects of algebra. In particular, we will work on bounded low/high sets, degrees of orders on torsion-free abelian groups, and reverse mathematics of several classic results in modules.

In Part I, we will study bounded-low sets and bounded-high sets in terms of the high/low hierarchy. Anderson and Csima proposed in [1] the notion of boundedjump on wtt-degrees, and proved an analogue of Shoenfield's jump inversion theorem. In [2], Anderson, Csima and Lange compared the bounded jump with Turing jump and proved the existence of high bounded-low sets and low bounded-high sets. Observing that superlow sets are all bounded-low, Anderson, Csima and Lange asked in [2] whether there exist bounded-low c.e. sets which are low but not superlow, and whether there exist superhigh sets which are not bounded-high. In Part I, we will provide positive answers to these questions. We will also prove that there are bounded-high sets which are high but not superhigh, and that there are bounded-low c.e. sets which are high but not superhigh.

In Part II, we study Turing degrees of orders on computable torsion-free abelian groups with infinite rank. Recently, Kach, Lange and Solomon [3] built a noncomputable c.e. set $C$ and a computable torsion-free abelian group $\mathscr{G}$ with computable orders such that every $C$-computable order on the constructed group is computable. Motivated by this result, we call a degree a group-order-computable if there is a computable torsion-free abelian group $\mathscr{G}$ with infinite rank which admits computable orders such that every a-computable order on $\mathscr{G}$ is computable; in this case, say a is group-order-computable via $\mathscr{G}$. Following this definition, we call a degree a weakly group-order-computable if there is a computable torsion-free abelian group $\mathscr{G}$ with 
infinite rank such that every a-computable group order on $\mathscr{G}$ is computable. We will prove that a Turing degree $\mathbf{a}$ is group-order-computable iff $\mathbf{a}$ is weakly grouporder-computable iff $\mathbf{a}$ is not a PA degree. In particular, all c.e. degrees except $\mathbf{0}^{\prime}$ are group-order-computable.

The objective to study group-order-computable degrees is indeed to explore degrees of orders on computable torsion-free abelian groups, because for a nonzero degree $\mathbf{a}$, if it is group-order-computable via $\mathscr{G}$, then $\mathscr{G}$ has no orders of degree a. We show that for any nonzero c.e. degree a, there is a computable torsionfree abelian group $\mathscr{G}$ and a nonzero c.e. degree $\mathbf{c}<\mathbf{a}$ such that $\mathscr{G}$ has orders of degree $\geq \mathbf{a}$ and $\mathbf{c}$ is group-order-computable via $\mathscr{G}$, which means that $\mathscr{G}$ has no incomputable orders of degree $\leq \mathbf{c}$.

In Part III, we study the reverse mathematics of classic results of divisible abelian groups and modules. We will prove that over $\mathrm{RCA}_{0}$, the decomposition theorem of divisible abelian groups and the decomposition theorem of torsion abelian groups are both equivalent to $\mathrm{ACA}_{0}$. We then consider projective modules and injective modules over $\Sigma_{1}^{0}$-principal ideal domains $\left(\Sigma_{1}^{0}\right.$-PIDs). We will also show that $\mathrm{ACA}_{0}$ proves the following theorems:

- every projective module over a $\Sigma_{1}^{0}$-PID is free;

- every submodule of a projective module over a $\Sigma_{1}^{0}$-PID is projective;

- every divisible module over a $\Sigma_{1}^{0}$-PID is injective;

- every quotient of an injective module over a $\Sigma_{1}^{0}$-PID is injective. 


\section{Chapter 1}

\section{Introduction}

\subsection{Basics of computability theory}

We first give a brief introduction of basic concepts and theorems in computability theory. Say that a function $f$ with domain $X \subseteq \mathbb{N}$ is partial computable if there is a Turing program $P$ such that for $x \in X, P$ running on input $x$ stops after finitely many steps, with output $f(x)$; and for $x \notin X, P$ running on input $x$ never stops. A subset of natural numbers is computably enumerable (c.e. for short) if it is the domain of a partial computable function. A subset $A$ of natural numbers is computable if both $A$ and its complement are computably enumerable.

Turing programs can be effectively listed, i.e. via their Gödel numbers, as $\left\{P_{e}\right.$ : $e \in \mathbb{N}\}$. Let $\varphi_{e}$ be the partial computable function computed by the $e$-th Turing program $P_{e}$, and $W_{e}$ be the domain of $\varphi_{e}$. Then $\left\{\varphi_{e}: e \in \mathbb{N}\right\}$ and $\left\{W_{e}: e \in \mathbb{N}\right\}$ are effective enumerations of partial computable functions and c.e. sets respectively. Similarly, we have an effective listing $\left\{\varphi_{e}^{k}: e \in \mathbb{N}\right\}$ of all $k$-ary partial computable functions with $k \geq 2$.

Lemma 1.1 (Padding Lemma) For each partial computable function $\varphi_{e}$, we can effectively find an index $e^{\prime}>$ e such that $\varphi_{e^{\prime}}=\varphi_{e}$.

Theorem 1.1 (Parameter Theorem) For a partial computable binary function, say $g$, there is an injective computable function s such that for all $x, y, \varphi_{s(x)}(y)=g(x, y)$.

Theorem 1.2 (Kleene's Recursion Theorem) For any total computable function $f: \mathbb{N} \rightarrow \mathbb{N}$, there is an $e_{0}$ such that $W_{e_{0}}=W_{f\left(e_{0}\right)}$. 
Theorem 1.3 (Recursion Theorem with parameters, Kleene) For any total computable function $f: \mathbb{N}^{2} \rightarrow \mathbb{N}$, there is a computable function $g: \mathbb{N} \rightarrow \mathbb{N}$ such that for any $n, W_{g(n)}=W_{f(n, g(n))}$.

Similar to Turing programs, all Turing programs with oracles can be effectively listed, and we have a standard enumeration $\left\{\Phi_{e}: e \in \mathbb{N}\right\}$ of partial computable Turing functionals. For any set $X$ and number $x$, we write $\Phi_{e}^{X}(x) \downarrow=y$ if $y$ is the output of the program $\Phi_{e}$ with oracle $X$ and input $x$. The use of this computation $\Phi_{e}^{X}(x)$, denoted by $\phi_{e}^{X}(x)$, is defined as the greatest number $z$ such that the question $z \in X$ or not is queried.

Theorem 1.4 (Parameter Theorem for functionals) For any partial computable binary functional, say $\Theta$, there is an injective computable function s such that for any oracle $Y$, and for all $x, y, \Phi_{s(x)}^{Y}(y) \simeq \Theta^{Y}(x, y)$.

Theorem 1.5 (Recursion Theorem for functionals) For any total computable function $f: \mathbb{N}^{2} \rightarrow \mathbb{N}$, there is a computable function $g: \mathbb{N} \rightarrow \mathbb{N}$ such that for any oracle $Y$ and number $n, \Phi_{g(n)}^{Y}=\Phi_{f(n, g(n))}^{Y}$.

Say that a set $A$ is Turing reducible to $B$, written as $A \leq_{T} B$, if there is a Turing program with oracle $B$ that outputs membership of $A$, and that $A$ is Turing equivalent to $B$, written as $A \equiv_{T} B$, if $A \leq_{T} B$ and $B \leq_{T} A$. $\equiv_{T}$ is an equivalence relation over subsets of $\mathbb{N}$, and we call the equivalence class of $A$ the Turing degree of $A$, denoted by $\operatorname{deg}(A)$. That is, $\operatorname{deg}(A)=\left\{B \subseteq \mathbb{N}: B \equiv_{T} A\right\}$. We also use a to denote the Turing degree of $A$. $\leq_{T}$ gives rise to a natural order $\leq$ among Turing degrees, i.e., $\mathbf{a} \leq \mathbf{b}$ if there are sets $A \in \mathbf{a}$ and $B \in \mathbf{b}$ such that $A \leq_{T} B$. 0 denotes the Turing degree of computable sets. If a degree a contains c.e. sets, then we call this degree a c.e. degree.

In computability theory, we also consider strong reducibilities, like one-one reducibility $\left(\leq_{1}\right)$, many-one reducibility $\left(\leq_{m}\right)$, truth-table reducibility $\left(\leq_{t t}\right)$ and weaktruth-table reducibility $\left(\leq_{w t t}\right)$. Under thse strong reducibilities, we can define corresponding degrees: 
- many-one degree ( $m$-degree, for short) of $A$ is defined as $\left\{X \subseteq \mathbb{N}: X \equiv_{m}\right.$ $A\}$. Here, $X$ is many-one reducible to $Y$, denoted by $X \leq_{m} Y$, if there is a computable function $f$ such that for all $x, x \in X$ iff $f(x) \in Y$. Moreover, if $f$ is one-to-one, $X$ is said to be one-one reducible to $Y$, denoted by $X \leq_{1} Y$.

- truth-table degree (tt-degree, for short) of $A$ is defined as $\left\{X \subseteq \mathbb{N}: X \equiv_{t t} A\right\}$. Let $\left\{\sigma_{n}: n \in \mathbb{N}\right\}$ be an effective list of all truth tables, i.e., propositional formals obtained from atomic formals $n \in X$ with $n \in \mathbb{N}$. $X$ is truth-table reducible to $Y$, denoted by $X \leq_{t t} Y$, if there is a computable function $f$ such that for all $x, x \in X \Leftrightarrow Y \vDash \sigma_{f(x)}$.

- weak-truth-table degree (wtt-degree, for short) of $A$ is defined as $\{X \subseteq \mathbb{N}$ : $\left.X \equiv_{w t t} A\right\}$. Here, $X$ is weak-truth-table reducible to $Y$, denoted by $X \leq_{w t t} Y$, if there is an oracle Turing program, say $\Phi_{e}$, such that $X=\Phi_{e}^{Y}$ with use $\phi_{e}^{Y}$ recursively bounded (i.e., there is a computable function $f$ such that $f(x) \geq$ $\phi_{e}^{Y}(x)$ for all $\left.x\right)$. Weak-truth-table reducibility is also called bounded-Turing reducibility.

Turing's halting problem is one of the most fundamental results in computability theory and has played a great role in the further development of this area.

Theorem 1.6 (Halting problem) $K=\left\{e \in \mathbb{N}: \varphi_{e}(e) \downarrow\right\}$ is computably enumerable, but not computable.

It is known that all c.e. sets are one-one reducible to $K$ and Shoenfield's limit lemma shows that all sets Turing reducible to $K$ have effective approximations.

The definition of Turing jump operator is based on a relativization of the Halting problem. For a given set $A$, define $A^{\prime}$ as the set $\left\{e \in \mathbb{N}: \Phi_{e}^{A}(e) \downarrow\right\}$, and we call $A^{\prime}$ the Turing jump of $A$. The Jump Theorem says that $A \leq_{T} B$ iff $A^{\prime} \leq_{1} B^{\prime}$, so $A \equiv_{T} B$ implies $A^{\prime} \equiv_{T} B^{\prime}$, and hence the jump operator defined above is well-defined. If a is the degree of $A$, the degree of $A^{\prime}$ is called the jump of $\mathbf{a}$, and is denoted by $\mathbf{a}^{\prime}$. The degree of Halting problem is just the Turing jump of $\mathbf{0}$, and denoted by $\mathbf{0}^{\prime}$. Obviously, if $\mathbf{a}$ is a c.e. degree, then $\mathbf{a} \leq \mathbf{0}^{\prime}$. Friedberg's jump inversion theorem says that the Turing jump, as a mapping on Turing degrees, is surjective over $\mathbf{0}^{\prime}$. 
Let $A^{(0)}=A$ and $A^{(1)}=A^{\prime}$, the first jump of $A$. We then define by induction the $n$-th jump of $A, A^{(n)}$, as the Turing jump of $A^{(n-1)}$. Let $\varnothing$ denote the empty set. Below is the well-known high/low hierarchy in computability theory.

Definition $1.1 A$ set $A \leq_{T} \varnothing^{\prime}$ is called lown if $A^{(n)} \equiv_{T} \varnothing^{(n)}$, and high $h_{n}$ if $A^{(n)} \equiv_{T}$ $\varnothing^{(n+1)}$.

Definition 1.2 A degree $\mathbf{c} \leq \mathbf{0}^{\prime}$ is lown if $\mathbf{c}^{(n)}=\mathbf{0}^{(n)}$, and high if $^{(n)}=\mathbf{0}^{(n+1)}$.

low , high $_{1}$ are also called low, high respectively. So $A \leq_{T} \varnothing^{\prime}$ is low if $A^{\prime} \leq_{T} \varnothing^{\prime}$, and high if $A^{\prime} \geq_{T} \varnothing^{\prime \prime}$. Mohrherr [4] named superlow sets and superhigh sets via the tt-reducibility as: $A \leq_{T} \varnothing^{\prime}$ is superlow if $A^{\prime} \leq_{t t} \varnothing^{\prime}$, and superhigh if $A^{\prime} \geq_{t t} \varnothing^{\prime \prime}$.

Another hierarchy which is popular in computability theory is the Ershov hierarchy, or the difference hierarchy.

Definition 1.3 For $n \in \mathbb{N}$, a set $A$ is called n-c.e., if there is a computable function $f(x, s)$ such that for all $x$,

- $f(x, 0)=0$,

- $A(x)=\lim _{s \rightarrow \infty} f(x, s)$,

- $|\{s \in \mathbb{N}: f(x, s) \neq f(x, s+1)\}| \leq n$.

A degree is $n$-c.e. with $n \geq 1$ if it contains a $n$-c.e. set, and properly $n$-c.e. with $n \geq 2$ if it is $n$-c.e. but not $(n-1)$-c.e. 1-c.e. sets (or degrees) are just c.e. sets (or degrees), and 2-c.e. sets (or degrees) are often called d.c.e. sets (or degrees). Cooper constructed a properly d.c.e. degree in 1971 in his PhD thesis [5], and his constructions can be modified to show the existence of properly $n$-c.e. degrees for $n \geq 2$.

The set of all degrees is denoted by $\mathcal{D}$, while the set of all c.e. degrees is denoted by $\mathcal{E}$. The following Figure 1.1 (from Cooper [6]) provides some basic information of these two hierarchies.

Definition 1.4 $A$ set $A$ is called $\omega$-c.e., if there is a computable function $f(x, s)$ and a computable function $g$ such that 


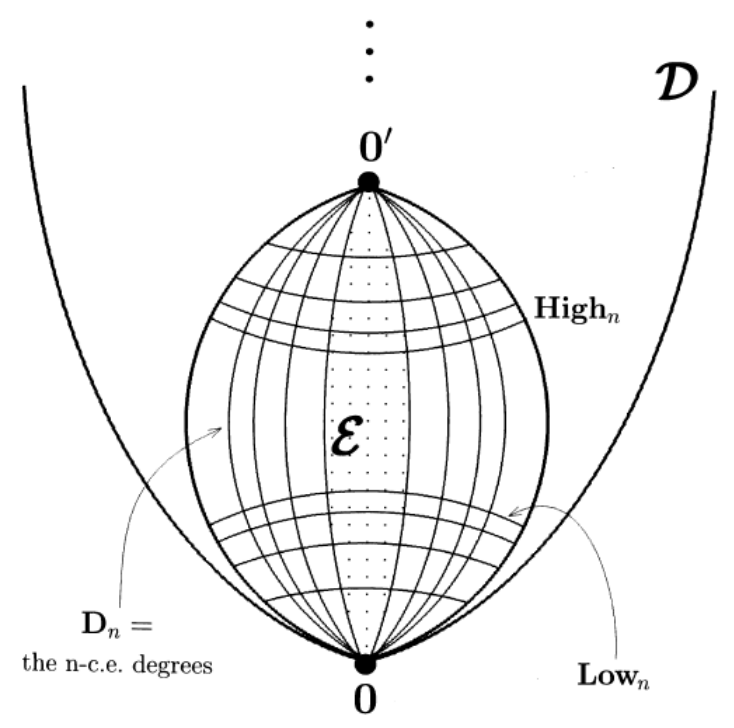

Figure 1.1: High/low hierarchy and Ershov hierarchy

- for all $x, A(x)=\lim _{s} f(x, s)$,

- for all $x,|\{s \in \mathbb{N}: f(x, s) \neq f(x, s+1)\}| \leq g(x)$.

The following relations between $\omega$-c.e. sets and strong reducibilities are wellknown:

Proposition 1.1 A set $A$ is $\omega$-c.e. iff $A \leq_{t t} \varnothing^{\prime}$ iff $A \leq_{w t t} \varnothing^{\prime}$.

The study of the structure of c.e. degrees stems from a question of Post, now known as the Post's problem:

Question 1.1 (Post (1944)) Does there exist c.e. degrees different from $\mathbf{0}$ and $\mathbf{0}^{\prime}$ ?

In order to solve his problem, Post himself developed many important concepts like simple sets, hypersimple sets and hyperhypersimple sets, with the attempt of making the complements of the constructed c.e. sets not c.e., by making them sparser and sparser. These concepts are closely related to strong reducibilities, in the sense that even though these sets can be Turing complete, simple sets can never be $m$-complete, and hypersimple sets can never be $t t$-complete.

Post's problem was eventually solved by Friedberg in 1957 and Muchnik in 1956, independently, where they introduced the injury method for the first time. 
Theorem 1.7 (Friedberg (1957), Muchnik (1956)) There exist c.e. sets $A$ and $B$ such that $A \not_{T} B$ and $B \not_{T} A$.

A solution for Post's problem, along Post's original idea of making the complements sparse, was found in the 1970s, after Ershov's work on $\eta$-maximal sets.

Friedberg and Muchnik's construction has a feature that the injuries from higher priority requirements are recursively bounded. In 1963, Sacks proved that any nonzero c.e. degree splits and this construction has an inherited feature that the injuries from higher priority requirements are finite, but cannot be bounded by any computable function.

Theorem 1.8 (Sacks Splitting Theorem (1963)) Let B be a noncomputable c.e. set. For any c.e. set $A$, there are low c.e. sets $C$ and $D$ such that

(1) $C \cap D=\varnothing, A=C \cup D$;

(2) $B \not_{T} C$ and $B \varliminf_{T} D$.

Theorem 1.8 says that any nonzero c.e. degree splits above $\mathbf{0}$. Robinson generalized Sacks splitting theorem to the splitting of a given c.e. degree above a low c.e. degree, which indicated that low c.e. degrees resemble degree $\mathbf{0 .}$

Theorem 1.9 (Robinson Splitting Theorem (1971) [7]) Let L be a low c.e. set. For any c.e. set $A>_{T} L$, there are c.e. sets $C$ and $D$ such that

(1) $C \cap D=\varnothing, A=C \cup D$;

(2) $A \not_{T} C \oplus L$ and $A \not \mathbb{K}_{T} D \oplus L$.

In 1964, Sacks proved that the c.e. degrees are dense, and the method used in the proof is an infinite injury argument. That is, the injuries from higher priority requirements can be infinite.

Theorem 1.10 (Sacks Density Theorem (1964)) For any two c.e. degrees $\mathbf{a}<\mathbf{b}$, there is a c.e. degree $\mathbf{c}$ such that $\mathbf{c} \in(\mathbf{a}, \mathbf{b})$. 
The same style of construction was used by Sacks to prove the existence of incomplete high c.e. degrees. In 1966, Lachlan introduced another kind of infinite injury arguments in his proof of the existence of minimal pairs. Here, a pair of nonzero c.e. degrees $\mathbf{a}$ and $\mathbf{b}$ form a minimal pair, denoted as $\mathbf{a} \cap \mathbf{b}=\mathbf{0}$, if $\mathbf{0}$ the infimum of $\mathbf{a}$ and $\mathbf{b}$, i.e., is the only c.e. degree below both $\mathbf{a}$ and $\mathbf{b}$.

Theorem 1.11 (Lachlan (1966), Yates (1966)) There are c.e. degrees forming a minimal pair.

Cooper proved in 1974 that any high c.e. degree bounds a minimal pair, and in this sense, high c.e. degrees resemble $\mathbf{0}^{\prime}$.

Theorem 1.12 (Cooper (1974) [8]) Any high c.e. degree bounds a minimal pair of c.e. degrees.

Miller proved in 1981 in his thesis [9] that any high c.e. degree bounds a minimal pair of high c.e. degrees. This work was published by Slaman and Shore in their paper [10], where a new construction was provided.

Definition 1.5 A c.e. degree $\mathbf{c}$ is cappable if it is zero or a half of a minimal pair, that is, there is a c.e. degree $\mathbf{a}>\mathbf{0}$ such that $\mathbf{c} \cap \mathbf{a}=\mathbf{0}$; otherwise, $\mathbf{c}$ is noncappable.

It is easy to prove that every nonzero c.e. degree bounds a nonzero cappable degree, which needs a nonuniform proof. The dual of cappable degrees is called cuppable degrees.

Definition 1.6 (1) A c.e. degree $\mathbf{c}$ is cuppable if there is a c.e. degree $\mathbf{a}<\mathbf{0}^{\prime}$ such that $\mathbf{c} \cup \mathbf{a}=\mathbf{0}^{\prime}$; otherwise, $\mathbf{c}$ is called noncuppable, i.e., for any c.e. degree $\mathbf{a}<\mathbf{0}^{\prime}, \mathbf{c} \cup \mathbf{a}<\mathbf{0}^{\prime}$.

(2) A c.e. degree $\mathbf{c}$ is called low-cuppable if there is a low c.e. $\mathbf{a}$ with $\mathbf{c} \cup \mathbf{a}=\mathbf{0}^{\prime}$.

Theorem 1.13 (Harrington (1976)) Any high c.e. degree bounds a high noncuppable degree. 
Ambos-Spies, Jockusch, Shore and Soare proved in [11] that cappable degrees form an ideal and noncappable degrees form a strong filter, providing a dichotomy of c.e. degrees.

Theorem 1.14 (Ambos-Spies, Jockusch, Shore and Soare [11]) A c.e. degree is noncappable iff it is low-cuppable (i.e., cups to $\mathbf{0}^{\prime}$ via a low c.e. degree).

Lachlan proved in 1975 that Sacks splitting theorem and Sacks density theorem cannot be combined, where he developed a new technique, now known as $\mathbf{0}^{\prime \prime \prime}$-arguments, a quite complicated construction of degrees.

Theorem 1.15 (Lachlan Nonsplitting Theorem (1975)) There are two c.e. degrees $\mathbf{a}<\mathbf{b}$ such that for any two c.e. degrees $\mathbf{c}, \mathbf{d}$, if $\mathbf{b}=\mathbf{c} \cup \mathbf{d}$, then $\mathbf{b} \leq \mathbf{a} \cup \mathbf{c}$ or $\mathbf{b} \leq \mathbf{a} \cup \mathbf{d}$

Harrington later proved in his paper Understanding Lachlan's monster paper that Lachlan's nonsplitting theorem can be strengthened as follows:

Theorem 1.16 (Harrington Nonsplitting Theorem (1980)) There exists a c.e. degree $\mathbf{a}<\mathbf{0}^{\prime}$ such that $\mathbf{0}^{\prime}$ cannot split above $\mathbf{a}$.

\subsection{Part I: Bounded-jump operator and the high/low hierarchy}

The notion of bounded-jump operator ${ }^{\dagger}$ was proposed by Anderson and Csima in paper [1], where they tried to find an appropriate jump operator on $w t t$-degrees ${ }^{1}$. For a set $A$, the bounded-jump of $A$ is defined as the set

$$
A^{\dagger}=\left\{e \in \mathbb{N}: \exists i \leq e\left[\varphi_{i}(e) \downarrow \& \Phi_{e}^{A\left\lceil\varphi_{i}(e)\right.}(e) \downarrow\right]\right\},
$$

where $A \uparrow_{z}:=\{x \in A: x \leq z\}$. Note that $A^{\dagger} \leq_{T} A \oplus \varnothing^{\prime}$. In [1], Anderson and Csima pointed out this bounded-jump operator ${ }^{\dagger}$ for $w t t$-reducibility behaves like the Turing jump ' for Turing reducibility, i.e., (1) $\varnothing^{\dagger}$ and $\varnothing^{\prime}$ are 1-equivalent, (2) for any set $A, A<_{w t t} A^{\dagger}$, and (3) for any sets $A, B$, if $A \leq_{w t t} B$, then $A^{\dagger} \leq_{w t t} B^{\dagger}$.

\footnotetext{
${ }^{1}$ In paper [1], Anderson and Csima used ${ }^{b}$ for the bounded-jump operator ${ }^{\dagger}$.
} 
(2) implies that $A^{\dagger} \equiv_{T} A$ when $A \geq_{T} \varnothing^{\prime}$. In the same paper, Anderson and Csima proved an analogue of Shoenfield's jump inversion theorem for this bounded-jump operator ${ }^{\dagger}$.

Say that a set $A$ is bounded-low if $A^{\dagger} \leq_{w t t} \varnothing^{\dagger}$, and bounded-high if $A \leq_{w t t} \varnothing^{\dagger}$ and $\varnothing^{\dagger \dagger} \leq{ }_{w t t} A^{\dagger}$. There are several immediate consequences: bounded-low sets and bounded-high sets are all $\omega$-c.e.; bounded-low sets are closed downwards under $w t t$-reducibility; and bounded-high sets are closed upwards under $w t t$-reducibility on $\omega$-c.e. sets. Furthermore, bounded-low sets cannot be bounded-high because $A^{\dagger} \leq_{w t t} \varnothing^{\dagger}$ implies $A^{\dagger}<_{w t t} A^{\dagger \dagger} \leq_{w t t} \varnothing^{\dagger \dagger}$.

It is easy to see that $A$ is bounded-low if and only if $A^{\dagger}$ is $\omega$-c.e. Thus, all superlow sets are bounded-low, because for all superlow sets $A, A^{\dagger} \leq_{1} A^{\prime} \leq_{t t} \varnothing^{\prime} \equiv_{1} \varnothing^{\dagger}$. Also note that $\varnothing^{\prime} \equiv_{1} \varnothing^{\dagger}$ is bounded-high, because $\varnothing^{\dagger \dagger} \leq_{w t t}\left(\varnothing^{\prime}\right)^{\dagger}$.

Proposition 1.2 (Bickford and Mills (1982) [12]) There are two superlow c.e. sets $A, B$ such that $A \oplus B \equiv_{T} \varnothing^{\prime}$.

The proposition above shows that there are two bounded-low sets whose join is bounded-high, thus not bounded-low. One natural question is:

Question 1.2 (1) Whether bounded-low sets are closed downwards under Turing reducibility?

(2) Similarly, whether bounded-high sets are closed upwards under Turing reducibility on $\omega$-c.e. sets?

For a superlow $A$, if $B \leq_{T} A$, then $B^{\prime} \leq_{1} A^{\prime} \leq_{t t} \varnothing^{\prime}$. Thus $B$ is also superlow. Superlow sets are closed downwards under Turing reducibility. Similarly, superhigh sets are closed upwards under Turing reducibility.

In Chapter 2, we will give negative answer to Question 1.2 by constructing a bounded low c.e. set which Turing computes $\varnothing^{\prime}$. This tells us that the greatest c.e. Turing degree $\mathbf{0}^{\prime}$ contains both bounded-low c.e. sets and bounded-high c.e. sets, and one easy consequence is:

Proposition 1.3 (1) There are bounded-low c.e. sets which are superhigh. 
(2) There are bounded-high c.e. sets which are superhigh.

Recently, in [2], Anderson, Csima and Lange initiated the study of comparing the bounded-jump and the Turing jump, where they constructed high bounded-low sets, and low bounded-high sets, showing that there is no direct relation between these two jumps. Motivated by the fact that superlowness implies bounded-lowness, they asked the following related questions:

Question 1.3 (Anderson, Csima and Lange [2])

(1) Does there exist a bounded-low set which is low but not superlow?

(2) Does there exist a bounded-high set which is high but not superhigh?

(3) Does there exist a superhigh set which is not bounded-high?

Question 1.3.(3) has positive answer because of the existence of bounded-low c.e. sets which are superhigh.

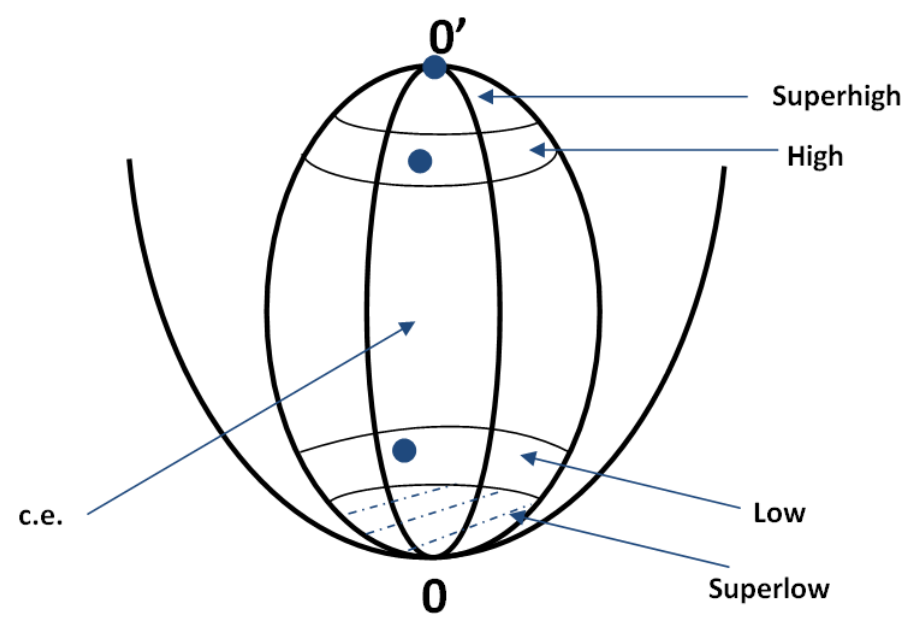

Figure 1.2: Turing degrees of bounded-low sets

We will also provide positive answers to the other two questions. In chapter 2 , we will construct a bounded-low c.e. set which is low but not superlow directly. In chapter 3 , we show the existence of bounded-high sets which are high but not superhigh by proving a pseudo-jump inversion theorem for bounded-high sets; we will then prove a pseudo-jump inversion theorem for bounded-low c.e. sets, and this implies the existence of bounded-low c.e. sets which are high but not superhigh. 


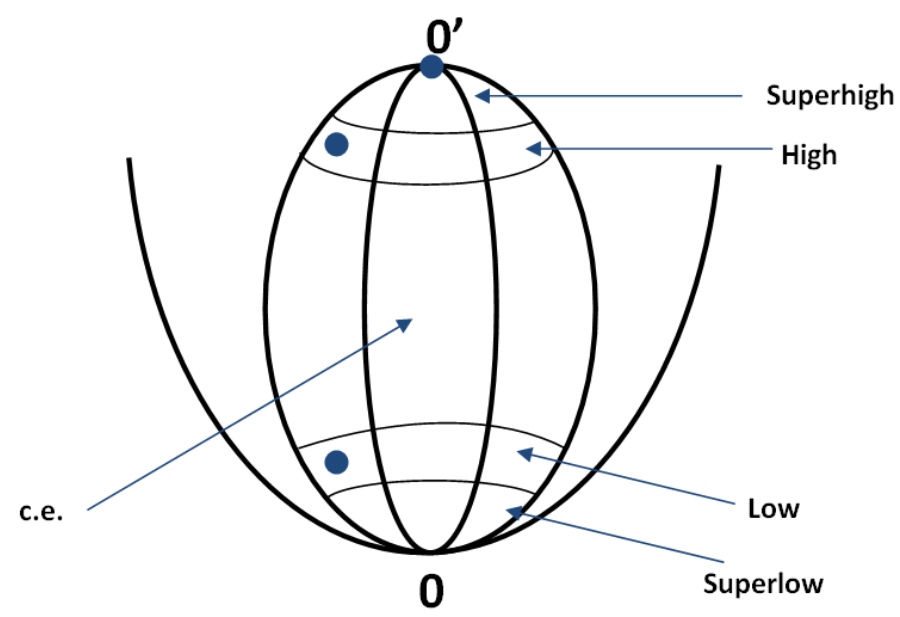

Figure 1.3: Turing degrees of bounded-high sets

Finally, we point out here that all bounded-high sets we constructed are $\omega$-c.e., and that $\varnothing^{\prime}$ is the only bounded-high c.e. set we know. It seems not easy to construct bounded-high c.e. sets, the questions related to constructions of bounded-high c.e. sets are still open as far as we know. For example, the following two questions still open.

Question 1.4 (Anderson and Csima [1]) Whether the analogue of Sacks's jump inversion is true or not? That is, for any set $A$ with $\varnothing^{\dagger} \leq_{w t t} A \leq_{w t t} \varnothing^{\dagger \dagger}$, does there exist a c.e. set $C \leq_{w t t} \varnothing^{\dagger}$ such that $C^{\dagger} \equiv_{w t t} A$ ?

Question 1.5 (Anderson, Csima and Lange [2]) Does there exist a low c.e. set which is bounded-high?

\subsection{Part II: Degrees of orders on torsion-free abelian groups}

An abelian group $\mathscr{G}=\left(G,+\mathscr{G}, 0_{\mathscr{G}}\right)$ is orderable if there is a linear order $\leq_{\mathscr{G}}$ on $G$ such that for all $x, y, z$ in $G, x \leq_{\mathscr{G}} y$ implies $x+\mathscr{G} z \leq_{\mathscr{G}} y+\mathscr{G} z$; say $\leq_{\mathscr{G}}$ is a group order (or simply order) on $\mathscr{G}$. It is known that an abelian group is orderable iff it is torsion-free (Levi's theorem). So we will restrict ourselves to torsion-free abelian groups.

From an order $\leq_{\mathscr{G}}$ on $\mathscr{G}$, one can form the set $P_{\leq \mathscr{G}}:=\left\{g \in G: g \geq_{\mathscr{G}} 0_{\mathscr{G}}\right\}$ of all positive elements under $\leq_{\mathscr{G}} . P_{\leq_{\mathscr{G}}}$ is a positive cone of $\leq_{\mathscr{G}}$. On the other hand, if 
$X \subseteq G$ satisfies the following conditions: (1) $X$ is a semigroup, (2) $X \cap-X=\left\{0_{\mathscr{G}}\right\}$, (3) $X \cup-X=G$, where $-X=\{-g: g \in X\}$, then we can define an order $\leq_{X}$ by

$$
x \leq_{X} y \Leftrightarrow y-x \in X
$$

and $X$ is the positive cone of the order $\leq_{X}$. Indeed, this entails a one-to-one correspondence between orders and positive cones on $\mathscr{G}$.

An abelian group $\mathscr{G}=\left(G,+\mathscr{G}, 0_{\mathscr{G}}\right)$ is computable if its domain $G$ and addition $+\mathscr{G}$ are both computable. Note that under this definition, the inverse function on $G$ is computable. If $\mathscr{G}$ is a computable torsion-free abelian group, we will use $\mathbb{X}(\mathscr{G})$ to denote the set of positive cones of $\mathscr{G}$, and we will study Turing degrees of members of $\mathbb{X}(\mathscr{G})$. Note that for such a group $\mathscr{G}, P_{\leq_{\mathscr{G}}}$ and $\leq_{\mathscr{G}}$ are Turing equivalent.

Definition 1.7 A class $\mathcal{C}$ of sets is called a $\Pi_{1}^{0}$ class if it can be represented as the set of all infinite paths of some computable binary tree.

For a computable torsion-free abelian group $\mathscr{G}$, the positive cones of $\mathscr{G}$ are defined via $\Pi_{1}^{0}$ conditions, and one can show that $\mathbb{X}(\mathscr{G})$ can be represented as the set of all infinite paths of a computable binary tree, which means that $\mathbb{X}(\mathscr{G})$ is a $\Pi_{1}^{0}$ class.

We are mainly interested in the degrees of members of $\mathbb{X}(\mathscr{G})$, i.e., the degrees of orders on $\mathscr{G}$. Classical results on degrees of members of $\Pi_{1}^{0}$ classes are due to Jockusch and Soare [13] in 1972, where the low basis theorem and the hyperimmunefree basis theorem are provided. These two basis theorems imply that every computable torsion-free abelian group has orders of low degree, and of hyperimmune-free degree, respectively.

Definition 1.8 For a torsion-free abelian group $\left(G,+, 0_{G}\right), g_{0}, \cdots g_{n} \in G$ are linearly independent if for any $a_{0}, \cdots a_{n} \in \mathbb{Z}$,

$$
a_{0} g_{0}+\cdots+a_{n} g_{n}=0_{G} \rightarrow a_{0}=\cdots=a_{n}=0 \text {. }
$$

An infinite subset $B$ of $G$ is linearly independent if any finite subset of $B$ is linearly independent. A maximal linearly independent set is called a basis of $G$. 
For $1 \leq k \leq \infty$, let $\mathbb{Q}^{k}$ denote the direct sum of $k$ many copies of additive group $\mathbb{Q}$. In particular, $\mathbb{Q}^{\infty}=\underset{i \in \mathbb{N}}{\oplus} \mathbb{Q}$, its elements are just finite $\mathbb{Q}$-linear sums over a computable basis $\left\{e_{i}: i \in \mathbb{N}\right\}$, where $e_{i}=(\cdots, 1,0, \cdots)$ is the sequence whose $i$-th column is 1 and all other columns are 0 .

The rank of $G, \operatorname{rank}(G)$, is defined as the size of a basis which is the least number $k \leq \infty$ such that $G$ embeds into $\mathbb{Q}^{k}$. Here are some easy examples:

- $\operatorname{rank}(\mathbb{Z})=\operatorname{rank}(\mathbb{Q})=1$.

- $\operatorname{rank}(\mathbb{Z} \oplus \mathbb{Z})=\operatorname{rank}(\mathbb{Q} \oplus \mathbb{Q})=2$.

- Let $G$ be the subgroup of $\mathbb{Q} \oplus \mathbb{Q}$ generated by $\left\{(1,0),(0,1),\left(\frac{1}{2}, \frac{1}{2}\right)\right\}$. Elements of $G$ are of the form $m(1,0)+n(0,1)+k\left(\frac{1}{2}, \frac{1}{2}\right)$ with $m, n, k \in \mathbb{Z}$. Then $\operatorname{rank}(G)=$ 2 .

We use $\mathcal{O}(\mathscr{G})$ to denote the set of all degrees of orders on a computable torsionfree abelian group $\mathscr{G}$. Solomon [14] proved the following results about $\mathcal{O}(\mathscr{G})$ :

(1) if $\operatorname{rank}(\mathscr{G})=1$, then $\mathcal{O}(\mathscr{G})$ contains exactly one degree $\mathbf{0}$;

(2) if $2 \leq \operatorname{rank}(\mathscr{G})<\infty$, then $\mathcal{O}(\mathscr{G})$ contains all degrees;

(3) if $\operatorname{rank}(\mathscr{G})=\infty$, then $\mathcal{O}(\mathscr{G})$ contains all degrees $\geq \mathbf{0}^{\prime}$.

For a computable torsion-free abelian group $\mathscr{G}$ and a $\Pi_{1}^{0}$ class $\mathcal{C}$, we say $\mathbb{X}(\mathscr{G})$ degree represents $\mathcal{C}$ if

$$
\mathcal{O}(\mathscr{G})=\{\operatorname{deg}(C): C \in \mathcal{C}\}
$$

where $\operatorname{deg}(C)$ is the Turing degree of $C$. Jockusch and Soare in [13] constructed an infinite $\Pi_{1}^{0}$ class, say $\mathcal{S}$, in which any two distinct elements are Turing incomparable. Then for any computable torsion-free abelian group $\mathscr{G}, \mathcal{O}(\mathscr{G}) \neq\{\operatorname{deg}(C): C \in \mathcal{S}\}$, because either $\mathcal{O}(\mathscr{G})=\{\mathbf{0}\}$ or $\mathcal{O}(\mathscr{G}) \supseteq\left\{\mathbf{d}: \mathbf{d} \geq \mathbf{0}^{\prime}\right\}$.

It turns out that the $\Pi_{1}^{0}$ class of positive cones on computable torsion-free abelian groups cannot degree represent all $\Pi_{1}^{0}$ classes. However, an earlier result of Metakides and Nerode [15] in 1979 showed that any $\Pi_{1}^{0}$ class can be degree represented by the $\Pi_{1}^{0}$ class of positive cones on some computably formally real field in a much stronger manner. 
For a computable torsion-free abelian group $\mathscr{G}$ with finite rank, we know that $\mathcal{O}(\mathscr{G})$ is either $\{\mathbf{0}\}$ or $\mathcal{D}$, the set of all degrees. However, for a computable torsionfree abelian group $\mathscr{G}$ with infinite rank, although $\mathcal{O}(\mathscr{G}) \supseteq\left\{\mathbf{d}: \mathbf{d} \geq \mathbf{0}^{\prime}\right\}$, we don’t know what the set $\mathcal{O}(\mathscr{G})$ exactly is. Here are two related results:

- (Solomon [14]) If $B$ is a basis of $\mathscr{G}$, then $\{\mathbf{d}: \mathbf{d} \geq \operatorname{deg}(B)\} \subseteq \mathcal{O}(\mathscr{G})$.

- (Kach, Lange, Solomon and Turetsky [3]) $\mathcal{O}(\mathscr{G})$ must contain infinitely many low degrees as well as infinitely many hyperimmune-free degrees.

In [3], Kach, Lange and Solomon considered the question whether $\mathcal{O}(\mathscr{G})$ is upwards closed for a computable torsion-free abelian group $\mathscr{G}$ with infinite rank, and provided a negative answer to it.

Theorem 1.17 (Kach, Lange and Solomon [3]) There is a computable torsion-free abelian group $\mathscr{G}$ with infinite rank, and a noncomputable c.e. set $C$ such that $\mathscr{G}$ has exactly two computable orders and every $C$-computable order on $\mathscr{G}$ is computable.

$\mathscr{G}$ in Theorem 1.17 has no orders in $\operatorname{deg}(C)$, but has orders in $\mathbf{0}$. By $\operatorname{deg}(C)>\mathbf{0}$, $\mathcal{O}(\mathscr{G})$ is not upwards closed.

Definition 1.9 A set $C$ is called group-order-computable via $\mathscr{G}$ if $\mathscr{G}$ is a computable torsion-free abelian group with infinite rank which admits computable orders, and every $C$-computable order on $\mathscr{G}$ is computable. $C$ is group-order-computable if it is group-order-computable via some $\mathscr{G}$.

Definition 1.10 A Turing degree $\mathbf{c}$ is called group-order-computable if it contains a set $C$ which is group-order-computable.

$\mathbf{0}$ is obviously group-order-computable, and group-order-computable degrees are downwards closed. On the one hand, Kach, Lange and Solomon's theorem above implies the existence of nonzero c.e. group-order-computable degrees, and hence can be low. In his thesis [16], C. J. Martin showed the existence of high c.e. degrees which are group-order-computable. On the other hand, any $\mathbf{a} \geq \mathbf{0}^{\prime}$ cannot be grouporder-computable because every computable torsion-free abelian group with infinite rank has an order of degree $\mathbf{a}$.

Our main objective in chapter 4 is to investigate the characterization of grouporder-computable degrees. 
Definition 1.11 $A$ set $C$ is said to be weakly group-order-computable via a computable torsion-free abelian group $\mathscr{G}$ with infinite rank if every $C$-computable order on $\mathscr{G}$ is computable. $C$ is weakly group-order-computable if $C$ is weakly group-ordercomputable via some $\mathscr{G}$.

A Turing degree is weakly group-order-computable if it contains a weakly grouporder-computable set. Clearly, group-order-computable degrees are weakly grouporder-computable. We will show that these two families of degrees, and also nonPA-degrees, coincide.

A set is called PA-complete if it computes a complete extension of Peano Arithmetic. An equivalent definition of PA-complete sets is that:

- a set $A$ is PA-complete iff it computes a $\{0,1\}$-valued diagonally nonrecursive function,

where a function $f$ is diagonally nonrecursive if $\forall x\left[\varphi_{x}(x) \downarrow \rightarrow f(x) \neq \varphi_{x}(x)\right]$. A degree is PA if it contains a PA-complete set. The notion of PA-degrees plays a vital role in the study of randomness. For example, Stephan [17] proved that a PA degree $\mathbf{a}$ is Martin-Löf random iff $\mathbf{a} \geq \mathbf{0}^{\prime}$.

The following facts for PA-degrees are well-known:

Proposition 1.4 (Scott and Solovay [18]) Let a be a Turing degree. The following are equivalent:

(1) a computes a complete extension of Peano Arithmetic.

(2) a computes a consistent extension of Peano Arithmetic.

(3) Each $\Pi_{1}^{0}$ class contains an a-computable element.

We first characterize weakly group-order-computable degrees in terms of PAdegrees.

Proposition 1.5 Let a be a Turing degree. The following are equivalent.

(1) a is a PA degree. 
(2) a is not weakly group-order-computable.

Proof: $(1) \Rightarrow(2)$. Let a be a PA degree, and $\mathscr{G}$ be a computable torsion-free abelian group with infinite rank (not necessarily possessing computable orders). We only need to show that $\mathscr{G}$ has an a-computable order which is not computable.

Martin proved in $[16]$ that $\mathbb{X}(\mathscr{G})$ has a $\Pi_{1}^{0}$ subclass, say $\mathcal{C}(\mathscr{G})$, containing no computable orders. As a computes a positive cone in $\mathcal{C}(\mathscr{G}), \mathscr{G}$ has an a-computable order which is not computable.

$(2) \Rightarrow(1)$. We first mention a result of Hatzikiriakou and Simpson [19] in reverse mathematics: the statement "every countable torsion-free abelian group is orderable" is equivalent to $\mathrm{WKL}_{0}$ over $\mathrm{RCA}_{0}$. This result has a consequence that there is a computable torsion-free abelian group, say $\mathscr{G}_{0}$, with a property that all orders of it are of PA degree. So $\mathscr{G}_{0}$ has no computable orders, hence the rank of $\mathscr{G}_{0}$ is infinite.

By definition, a degree $\mathbf{a}$ is not weakly group-order-computable, if any computable torsion-free abelian group with infinite rank has an a-computable order which is not computable. In particular, $\mathscr{G}_{0}$ has an a-computable order, and hence a computes an order on $\mathscr{G}_{0}$, which is of PA degree. This implies that a itself is a PA degree.

By Proposition 1.5, all PA-degrees are not group-order-computable. We will construct in chapter 4 a computable torsion-free abelian group $\mathscr{G}$ with infinite rank such that for any set $A, \mathscr{G}$ admits exactly two $A$-computable orders iff $A$ is not PA-complete.

As computable sets are not PA-complete, $\mathscr{G}$ above admits exactly two computable orders. Then for any set $A$ which is not PA-complete, every $A$-computable order on $\mathscr{G}$ is computable; for any set $A$ which is PA-complete, $A$ computes a noncomputable order on $\mathscr{G}$. This shows that for any degree a, if it is now not group-order-computable, then it is a PA degree. Hence, a degree is PA iff it is not weakly group-order-computable iff it is not group-order-computable.

At the end of [3], by applying the low basis theorem of $\Pi_{1}^{0}$ classes infinitely many times, Kach, Lange, Solomon and Turetsky showed that every computable torsionfree abelian group of infinite rank has orders of infinitely many low degrees. We would like to know whether $\mathscr{G}$ in Theorem 1.17 has orders of other degrees: 
Question 1.6 Given a nonzero c.e. degree $\mathbf{a}$, can we build a group $\mathscr{G}$ such that: (1) it has exactly two computable orders, (2) $\mathscr{G}$ has an order of degree $\mathbf{a}$, and (3) there is a noncomputable c.e. $C$ such that every $C$-computable group order on $\mathscr{G}$ is computable?

We will give a positive answer to Question 1.6 in chapter 5. Our results show that for any nonzero c.e. degree $\mathbf{a}$, there is a nonzero c.e. degree $\mathbf{c}<\mathbf{a}$, and a computable torsion-free abelian group $\mathscr{G}$ such that $\mathscr{G}$ has orders of degree $\geq$ a but has no incomputable orders of degree $\leq \mathbf{c}$.

\subsection{Part III: Reverse mathematics, abelian groups and modules}

In this part, we study reverse mathematics of some classic theorems in module theory. We first briefly introduce common subsystems of second order arithmetic studied in reverse mathematics. $L_{2}$, the language of second order arithmetic, contains two-sorted variables, namely, number variables and set variables. The full system $Z_{2}$ of second order arithmetic contains basic axioms of ordered semiring of natural numbers, induction axioms of sets, i.e., for any set $X$,

$$
(0 \in X \wedge(n \in X \rightarrow n+1 \in X)) \rightarrow \forall n(n \in X)
$$

and comprehension axioms of formulas $\varphi(n)$ in $L_{2}$, i.e.,

$$
\exists X \forall n(n \in X \leftrightarrow \varphi(n))
$$

where $X$ does not occur freely in $\varphi$.

Many theorems in non-set-theoretic mathematics are classified by five big subsystems of $Z_{2}$, i.e., $\mathrm{RCA}_{0}, \mathrm{WKL}_{0}, \mathrm{ACA}_{0}, \mathrm{ATR}_{0}$ and $\Pi_{1}^{1}-\mathrm{CA}_{0}$. Simpson's book [20] provides a comprehensive introduction of these five subsystems. We introduce $\mathrm{RCA}_{0}$ and $\mathrm{ACA}_{0}$ in detail here, as we will mainly work with these two subsystems.

$\mathrm{RCA}_{0}$ contains basic axioms of ordered semiring of natural numbers, $\Sigma_{1}^{0}$-induction and $\Delta_{1}^{0}$-comprehension. $\Sigma_{1}^{0}$-induction is the axiom of the following form with $\varphi$ restricted to $\Sigma_{1}^{0}$ formulas:

$$
(\varphi(0) \wedge(\varphi(n) \rightarrow \varphi(n+1))) \rightarrow \forall n \varphi(n)
$$


$\Delta_{1}^{0}$-comprehension is the axiom of the following form with $\varphi$ restricted to $\Delta_{1}^{0}$ formulas:

$$
\exists X \forall n(n \in X \leftrightarrow \varphi(n)),
$$

where $X$ does not occur freely in $\varphi$.

$\mathrm{ACA}_{0}$ contains basic axioms of ordered semiring of natural numbers, induction axioms of sets, and $\Sigma_{k}^{0}$-comprehension for all $k$. $\Sigma_{k}^{0}$-comprehension axioms say

$$
\exists X \forall n(n \in X \leftrightarrow \varphi(n)),
$$

where $\varphi$ is a $\Sigma_{k}^{0}$-formula and $X$ does not occur freely in $\varphi(n)$.

The following theorem is widely used in reverse mathematics when proving a theorem in ordinary mathematics implies $\mathrm{ACA}_{0}$.

Theorem 1.18 The following are equivalent over $\mathrm{RCA}_{0}$.

(1) $\mathrm{ACA}_{0}$;

(2) Let $f: \mathbb{N} \rightarrow \mathbb{N}$ be a one-to-one function, then the range of $f$ exists.

We now define basic concepts of module theory using the language $L_{2}$ of second order arithmetic.

Definition $1.12\left(\mathrm{RCA}_{0}\right)$ Let $R$ be a commutative ring with identity $1_{R}$, a module $M$ over $R$ is an abelian group together with a scalar operation $\circ$ from $R \times M$ to $M$, satisfying for all $m, m_{1}, m_{2} \in M$ and $r, r_{1}, r_{2} \in R$,

M1. $\left(r_{1}+r_{2}\right) \circ m=r_{1} \circ m+r_{2} \circ m ;$

M2. $\left(r_{1} r_{2}\right) \circ m=r_{1} \circ\left(r_{2} \circ m\right)$;

M3. $1_{R} \circ m=m$;

M4. $r \circ\left(m_{1}+m_{2}\right)=r \circ m_{1}+r \circ m_{2}$.

For convenience, we write $r m$ for $r \circ m$.

Definition $1.13\left(\mathrm{RCA}_{0}\right)$ Let $M_{1}$ and $M_{2}$ be two $R$-modules. $A$ map $\varphi: M_{1} \rightarrow M_{2}$ is a $R$-module homomorphism if for any $x, y \in M_{1}$ and $r \in R, \varphi(x+y)=\varphi(x)+\varphi(y)$, $\varphi(r x)=r \varphi(x)$. 
Definition $1.14\left(\mathrm{RCA}_{0}\right)$ Let $\varphi: A \rightarrow B$ and $\psi: B \rightarrow C$ be two $R$-module homomorphisms. The sequence $A \stackrel{\varphi}{\rightarrow} B \stackrel{\psi}{\rightarrow} C$ is exact at $B$ if $\operatorname{ker}(\psi)=i m(\varphi)$. Moreover, if $\varphi$ is a monomorphism and $\psi$ is a surjective homomorphism, then the sequence

$$
0 \rightarrow A \stackrel{\varphi}{\rightarrow} B \stackrel{\psi}{\rightarrow} C \rightarrow 0
$$

is also exact at $A, C$, and often called a short exact sequence.

We only consider countable modules over a commutative ring with identity and mainly deal with infinite modules whose domain are subsets of $\mathbb{N}$. Within $\mathrm{RCA}_{0}$, the set $\mathbb{N}<\mathbb{N}$ of all codes of finite sequences of natural numbers exists (see [20] for more details). The symbol $\left\langle x_{0}, x_{1}, \cdots, x_{n}\right\rangle$ denotes the unique code of a sequence of natural numbers of length $n+1$, we often identify a sequence with its code.

For a sequence $\sigma, \operatorname{lh}(\sigma)$ denotes the length of $\sigma$. We use $\lambda$ to denote the empty sequence, and $l h(\lambda)=0$. For a nonempty sequence $\sigma, \operatorname{lh}(\sigma) \geq 1$, and we often write $\sigma=\langle\sigma(0), \sigma(1), \cdots, \sigma(\operatorname{lh}(\sigma)-1)\rangle$. For two sequences $\sigma, \tau$, the concatenation $\sigma^{\curvearrowright} \tau$ of them is just the sequence $\langle\sigma(0), \sigma(1), \cdots, \sigma(\operatorname{lh}(\sigma)-1), \tau(0), \tau(1), \cdots, \tau(\operatorname{lh}(\tau)-1)\rangle$.

Definition $1.15\left(\mathrm{RCA}_{0}\right)$ For $0 \leq i \leq n$, let $\left(M_{i},+_{M i}, \circ_{M_{i}}, 0_{M_{i}}\right)$ be a $R$-module. The direct sum $\bigoplus_{0 \leq i \leq n} M_{i}$ of modules $M_{i}(0 \leq i \leq n)$ is a $R$-module containing finite sequences of length $n+1$ of the form $\left\langle m_{0}, m_{1}, \cdots, m_{n}\right\rangle$ with each $m_{i} \in M_{i}$, together with componentwise addition

$$
\left\langle m_{0}, \cdots, m_{n}\right\rangle+\left\langle m_{0}^{\prime}, \cdots, m_{n}^{\prime}\right\rangle=\left\langle m_{0}+{ }_{M_{0}} m_{0}^{\prime}, \cdots, m_{n}+{ }_{M_{n}} m_{n}^{\prime}\right\rangle
$$

for abelian groups and componentwise scalar multiplication

$$
r\left\langle m_{0}, \cdots, m_{n}\right\rangle=\left\langle r \circ_{M_{0}} m_{0}, \cdots, r \circ_{M_{n}} m_{n}\right\rangle
$$

for modules. The zero of $\bigoplus_{0 \leq i \leq n} M_{i}$ is $\left\langle 0_{M_{0}}, 0_{M_{1}}, \cdots, 0_{M_{n}}\right\rangle$. If $M_{i}=M$ for all $0 \leq i \leq$ $n$, we often use $M^{n+1}$ to denote $\bigoplus_{0 \leq i \leq n} M$.

In general, as in Solomon [21], we can define the direct sum of countably many $R$ modules as a module whose nonzero elements are finite sequences with last column nonzero, and zero element is just the empty sequence. 
Definition $1.16\left(\mathrm{RCA}_{0}\right)$ Let $M_{i}(i \in \mathbb{N})$ be R-modules. The direct sum $\bigoplus_{i \in \mathbb{N}} M_{i}$ is a R-module containing finite sequences of the form $\left\langle m_{0}, m_{1}, \cdots, m_{n}\right\rangle$ with $n \in \mathbb{N}$, $m_{i} \in M_{i}$ for $0 \leq i \leq n, m_{n} \neq 0_{M_{n}}$, and the empty sequence, together with componentwise addition and componentwise scalar multiplication. Empty sequence is the zero element of $\bigoplus_{i \in \mathbb{N}} M_{i}$. We simply write $\bigoplus_{i \in \mathbb{N}} M$ (or $M^{\infty}$ ) for $\bigoplus_{i \in \mathbb{N}} M_{i}$ when $M_{i}=M$ for all $i \in \mathbb{N}$.

Definition $1.17\left(\mathrm{RCA}_{0}\right) A$ short exact sequence $0 \rightarrow A \stackrel{\mu}{\rightarrow} B \stackrel{\varepsilon}{\rightarrow} C \rightarrow 0$ splits if there exists a homomorphism $\sigma: C \rightarrow B$ such that $\varepsilon \sigma$ is the identity homomorphism on $C . \sigma$ is called a splitting for this short exact sequence.

Lemma $1.2\left(\mathrm{RCA}_{0}\right)$ If the short exact sequence $0 \rightarrow A \stackrel{\mu}{\rightarrow} B \stackrel{\varepsilon}{\rightarrow} C \rightarrow 0$ splits with a splitting $\sigma: C \rightarrow B$, then $B \cong A \oplus C$. Moreover, $B=\mu(A) \oplus \sigma(C)$.

Proof: Define a map $\psi: A \oplus C \rightarrow B ;\langle a, c\rangle \mapsto \mu(a)+\sigma(c)$. We now show that $\psi$ is an isomorphism between $A \oplus C$ and $B$.

For any $a, a^{\prime} \in A, c, c^{\prime} \in C$, and $r \in R$,

$$
\begin{aligned}
\psi\left(\langle a, c\rangle+\left\langle a^{\prime}, c^{\prime}\right\rangle\right) & =\psi\left(\left\langle a+a^{\prime}, c+c^{\prime}\right\rangle\right) \\
& =\mu\left(a+a^{\prime}\right)+\sigma\left(c+c^{\prime}\right) \\
& =\mu(a)+\mu\left(a^{\prime}\right)+\sigma(c)+\sigma\left(c^{\prime}\right) \\
& =\psi(\langle a, c\rangle)+\psi\left(\left\langle a^{\prime}, c^{\prime}\right\rangle\right)
\end{aligned}
$$

and

$$
\psi(r\langle a, c\rangle)=\psi(\langle r a, r c\rangle)=\mu(r a)+\sigma(r c)=r \mu(a)+r \sigma(c)=r \psi(\langle a, c\rangle) .
$$

So $\psi$ is a $R$-module homomorphism.

$\psi$ is injective. First, $\psi(\langle a, c\rangle)=\mu(a)+\sigma(c)=0$ implies $0=\varepsilon(\mu(a)+\sigma(c))=$ $0+\varepsilon \sigma(c)=c$. Then $\mu(a)=0$, and this implies $a=0$. So $\langle a, c\rangle=0$.

$\psi$ is surjective. For any $b \in B, \varepsilon(b) \in C$ and $\psi(\langle 0, \varepsilon(b)\rangle)=\sigma \varepsilon(b), \varepsilon \sigma \varepsilon(b)=\varepsilon(b)$. So $\sigma \varepsilon(b)-b \in \operatorname{ker}(\varepsilon)=i m(\mu)$. Then there exists an $a \in A$ such that $\mu(a)=\sigma \varepsilon(b)-b$, and then $b=\mu(a)+\sigma \varepsilon(b)=\psi(\langle a, \varepsilon(b)\rangle)$. This also implies that $B=\mu(A)+\sigma(C)$.

Now we show $B=\mu(A) \oplus \sigma(C)$. It is enough to prove $\mu(A) \cap \sigma(C)=\{0\}$. Let $b \in B$. Assume $b=\mu(a)=\sigma(c)$ for some $a \in A$ and $c \in C$. Then $\psi(\langle a,-c\rangle)=$ 
$\mu(a)-\sigma(c)=0$. Similarly, $0=\varepsilon(\mu(a)-\sigma(c))=0-\varepsilon \sigma(c)=-c$. Then $c=0$, and thus $b=0$.

In Chapter 6, we first consider reverse mathematics of divisible abelian groups.

Definition $1.18\left(\mathrm{RCA}_{0}\right)$ Let $G$ be an abelian group, and $g \in G$.

(1) $g$ is torsion if there is a natural number $n \geq 1$ such that $n g=0_{G}$.

(2) The order $o(g)$ of an element $g \in G$ is the least natural number $n \geq 1$ such that $n g=0_{G}$ if $g$ is torsion, and $\infty$ otherwise.

An abelian group $G$ is torsion if any element of $G$ has a finite order, and torsionfree if any nonzero element of $G$ has an infinite order. Notions like torsion groups, torsion-free groups are all definable within $\mathrm{RCA}_{0}$.

Definition $1.19\left(\mathrm{RCA}_{0}\right)$ An abelian group $G$ is divisible if for every $g \in G$ and for every nonzero $n \in \mathbb{N}$, there is a $g^{\prime} \in G$ such that $g=n g^{\prime}$.

For a prime number $p, \mathbb{Z}\left(p^{\infty}\right)=\left\{\frac{n}{p^{m}}: m, n \in \mathbb{Z}\right\} / \mathbb{Z}$ exists in $\mathrm{RCA}_{0}$.

We will prove that over $\mathrm{RCA}_{0}$, the following are equivalent:

(1) $\mathrm{ACA}_{0}$

(2) Every divisible subgroup of an abelian group is a direct summand.

(3) Every torsion-free abelian group has the largest divisible subgroup.

(4) Every divisible abelian group is isomorphic to a direct sum of the form

$\bigoplus_{n \in I}\left(\mathbb{Z}\left(p_{n}^{\infty}\right)\right)^{l_{n}} \oplus \bigoplus_{n \in J} \mathbb{Q}$ for some $I, J \subseteq \mathbb{N}$, and if $I \neq \varnothing$, for each $n \in I$, $1 \leq l_{n} \leq \infty$.

For an $R$-module $M$ and a subset $A$ of $M$, the set

$$
\langle A\rangle=\left\{r_{0} a_{0}+\cdots+r_{n} a_{n}: n \in \mathbb{N}, 0 \leq i \leq n, r_{i} \in R, a_{i} \in A\right\}
$$

of $R$-linear combinations of elements of $A$ forms a submodule of $M$. If $M=\langle A\rangle$, we say $A$ generates $M$ or $A$ is a set of generators of $M$.

A finite set $\left\{m_{0}, m_{1}, \cdots, m_{n}\right\}$ of $M$ is linearly independent if for any $r_{i} \in R, 0 \leq$ $i \leq n, r_{0} m_{0}+\cdots+r_{n} m_{n}=0$ implies $r_{i}=0$ for all $0 \leq i \leq n$. An infinite subset $A$ of $M$ is linearly independent if any finite subset of $A$ is linearly independent. A $R$-module $M$ has a basis $B$ if $B$ is linearly independent and $B$ generates $M$. 
Definition $1.20\left(\mathrm{RCA}_{0}\right)$ A $R$-module is free if it has a basis.

Proposition $1.6\left(\mathrm{RCA}_{0}\right)$ Let $F$ be a free $R$-module with an infinite basis, say $B=$ $\left\{b_{i}: i \in \mathbb{N}\right\}$. Then $F$ is isomorphic to $\bigoplus_{i \in \mathbb{N}} R$.

Proof: Let $c_{i}=\left\langle 0, \cdots, 0,1_{R}\right\rangle \in \bigoplus_{i \in \mathbb{N}} R$ of length $i+1$. That is, the $j$-th column is the zero element of $R$ for $j \leq i-1$ and the $i$-th column is the identity of $R$. Then $\left\{c_{i}: i \in \mathbb{N}\right\}$ is a basis of $\bigoplus_{i \in \mathbb{N}} R$. Define $\varphi: F \rightarrow \bigoplus_{i \in \mathbb{N}} R$ by $\varphi\left(\sum_{0 \leq i \leq n} r_{i} b_{i}\right)=\sum_{0 \leq i \leq n} r_{i} c_{i}$ for any $n \in \mathbb{N}$ and $r_{i} \in R$ with $0 \leq i \leq n$.

Proposition $1.7\left(\mathrm{RCA}_{0}\right)$ Let $M$ be a $R$-module. Then $M$ is isomorphic to a quotient of the free module $F=\bigoplus_{i \in \mathbb{N}} R$.

Proof: List $M$ as $m_{0}, m_{1}, \cdots$. Define a map $\varphi: F \rightarrow M$ as follows: first, for all $i$, $\varphi\left(c_{i}\right)=m_{i}$, where $c_{i}=\left\langle 0, \cdots, 0,1_{R}\right\rangle$ of length $i+1$; second, extend $\varphi$ linearly to all elements of $F$. That is, $\varphi\left(\sum_{0 \leq i \leq n} r_{i} c_{i}\right)=\sum_{0 \leq i \leq n} r_{i} m_{i}$. As $\varphi$ is surjective, $M \cong F / k e r(\varphi)$, where $\operatorname{ker}(\varphi)=\{x \in F: \varphi(x)=0\}$.

Proposition $1.8\left(\mathrm{RCA}_{0}\right)$ Let $F=\bigoplus_{i \in \mathbb{N}} R$. For every surjective homomorphism $\varepsilon: B \rightarrow C$ of $R$-modules and every homomorphism $\varphi: F \rightarrow C$, there exists a homomorphism $\psi: F \rightarrow B$ such that $\varphi=\varepsilon \psi$.

Proof: Let $c_{0}, c_{1}, \cdots$ be a basis of $F$. For each $\varphi\left(c_{i}\right) \in C$, find an element $d_{i}$ of $B$ such that $\varepsilon\left(d_{i}\right)=\varphi\left(c_{i}\right)$. Define for all $i \in \mathbb{N}, \psi\left(c_{i}\right)=d_{i}$.

Definition $1.21\left(\mathrm{RCA}_{0}\right)$ Let $P$ be a $R$-module. $P$ is called projective if for every surjective homomorphism $\varepsilon: B \rightarrow C$ of $R$-modules and every homomorphism $\varphi$ : $P \rightarrow C$, there exists a homomorphism $\psi: P \rightarrow B$ such that $\varphi=\varepsilon \psi$.

The following proposition is an immediate consequence of Proposition 1.8.

Proposition 1.9 (Yamazaki [22])( $\left.\mathrm{RCA}_{0}\right)$ A free $R$-module is projective.

Theorem 1.19 (Yamazaki [22]) Over $\mathrm{RCA}_{0}$, a $R$-module is projective iff it is isomorphic to a direct summand of a free $R$-module. 
A free $R$-module is projective, but the converse fails in general. However, classically, for a principal ideal domain $R$, projective $R$-modules are always free.

Definition $1.22\left(\mathrm{RCA}_{0}\right) A$ commutative ring $R$ with identity $1_{R}$ is an integral domain if it has no zero divisors, that is, for any $a, b \in R-\{0\}, a b \neq 0$.

Downey, Lempp and Mileti in [23] showed that there is a computable integral domain, say $S$, which is not a field such that $S$ has no computable non-trivial proper ideals which are finitely generated. In particular, $S$ has no computable non-trivial proper ideals which are principal. Hence, within $\mathrm{RCA}_{0}$, one cannot show that any integral domain which is not a field has a principal non-trivial proper ideal. Indeed, Downey, Lempp, and Mileti [23] proved that the statement that "every commutative ring which is not a field has a finitely generated nontrivial proper ideal" is equivalent to $\mathrm{ACA}_{0}$ over $\mathrm{RCA}_{0}$. This result of Downey, Lempp, and Mileti also implies that "every commutative ring which is not a field has a principal non-trivial proper ideal" is equivalent to $\mathrm{ACA}_{0}$ over $\mathrm{RCA}_{0}$, refer to Sato's thesis [24] (Theorem 6.11).

In reverse mathematics, to prove basic properties on principal ideal domains (PIDs) even within weak systems like $\mathrm{RCA}_{0}$, we had better to define a PID as an integral ideal domain whose $\Sigma_{1}^{0}$-ideals are generated by one element. Such PIDs are known as $\Sigma_{1}^{0}$-PIDs, refer to Simpson [25] and Sato [24] for more details.

\section{Definition $1.23\left(\mathrm{RCA}_{0}\right)$}

(1) Let $R$ be a commutative ring with $1_{R}$. A sequence $\left\langle r_{n}: n \in \mathbb{N}\right\rangle \subseteq R$ is a $\Sigma_{1}^{0}$-ideal if for any $i, j \in \mathbb{N}$, there is a $k \in \mathbb{N}$ such that $r_{k}=r_{i}+r_{j}$ and for any $i \in \mathbb{N}$ and $s \in R$, there is a $j \in \mathbb{N}$ such that $r_{j}=r_{i} s$.

(2) An integral domain $R$ is a $\Sigma_{1}^{0}$-PID, if for any $\Sigma_{1}^{0}$-ideal $I$ of $R$, there is an element $r \in I$ such that $I=(r)$, the ideal generated by $r$.

We will prove in Section 7.1 some results related to projective modules within $\mathrm{ACA}_{0}$. In particular, we will show that $\mathrm{ACA}_{0}$ proves:

(1) every projective module over a $\Sigma_{1}^{0}$-PID is free;

(2) every submodule of a projective module over a $\Sigma_{1}^{0}$-PID is projective. 
As a dual to projective modules, we have the following definition for injective modules.

Definition $1.24\left(\mathrm{RCA}_{0}\right)$ Let $I$ be a $R$-module. $I$ is injective if for any monomorphism $\mu: A \rightarrow B$ and any homomorphism $\varphi: A \rightarrow I$, there exists a homomorphism $\psi: B \rightarrow I$ such that $\varphi=\psi \mu$.

The following classical lemma for injective modules is known as Baer's criterion.

Lemma 1.3 (Baer's Criterion) A module $I$ over a commutative ring $R$ with $1_{R}$ is injective iff for every ideal $J$ of $R$ and every $R$-module homomorphism $f: J \rightarrow I$, there is a homomorphism $g: R \rightarrow I$ such that $g \uparrow_{J}=f$.

Proposition $1.10\left(\mathrm{RCA}_{0}\right)$ A module $I$ over a commutative ring $R$ with $1_{R}$ is injective implies for every ideal $J$ of $R$ and every $R$-module homomorphism $f: J \rightarrow I$, there is a homomorphism $g: R \rightarrow I$ such that $g \uparrow_{J}=f$.

Theorem 1.20 (Yamazaki [22]) The following are equivalent over $\mathrm{RCA}_{0}$.

(1) $\mathrm{ACA}_{0}$

(2) If for every ideal $J$ of $R$ and every $R$-module homomorphism $f: J \rightarrow I$, there is a homomorphism $g: R \rightarrow I$ such that $g \uparrow_{J}=f$, then $I$ is injective.

Definition $1.25\left(\mathrm{RCA}_{0}\right)$ Let $R$ be an integral domain. $A$-module $D$ is divisible if for every $d \in D$ and nonzero $r \in R$, there is a $c \in D$ such that $d=r c$.

Classically, a module over a principal ideal domain is injective iff it is divisible.

Proposition $1.11\left(\mathrm{RCA}_{0}\right)$ An injective module $I$ over an integral domain $R$ is divisible.

Proof: For any nonzero $r \in R$ and $d \in I$, let $\mu: R \rightarrow R ; 1_{R} \mapsto r$ and $\varphi: R \rightarrow$ $I ; 1_{R} \mapsto d$. As $\mu\left(r^{\prime}\right)=r^{\prime} r=0$ implies $r^{\prime}=0, \mu$ is a monomorphism. As $I$ is an injective $R$-module, there is a homomorphism $\psi: R \rightarrow I$ such that $\varphi=\psi \mu$. Now $d=\varphi\left(1_{R}\right)=\psi \mu\left(1_{R}\right)=\psi(r)=r \psi\left(1_{R}\right)$ with $\psi\left(1_{R}\right) \in I$.

The following theorem about reverse mathematics of divisible abelian groups is due to Friedman, Simpson and Smith [26] and can be found in Simpson's book [20]. 
Theorem 1.21 [26] The following are equivalent over $\mathrm{RCA}_{0}$.

(1) $\mathrm{ACA}_{0}$

(2) Every divisible abelian group is injective.

In Section 7.2, we will extend Theorem 1.21 to modules, and show that the statement that "every divisible module over a $\Sigma_{1}^{0}$-PID is injective" is equivalent to $\mathrm{ACA}_{0}$ over $\mathrm{RCA}_{0}$. We will also show that $\mathrm{ACA}_{0}$ proves the statement that "every quotient of an injective module over a $\Sigma_{1}^{0}$-PID is injective". 


\section{Part I}

\section{Bounded-jump operator and the high/low hierarchy}




\section{Chapter 2}

\section{A bounded-low c.e. set which is low, but not superlow}

In this chapter, we will answer two questions raised by Anderson, Csima and Lange in [2] by proving: (1) there is a bounded-low c.e. set which is low, but not superlow; (2) $\mathbf{0}^{\prime}$ contains a bounded-low c.e. set.

Anderson, Csima and Lange considered in [2] the interaction between the boundedjump operator and the Turing jump, and constructed a high bounded-low set. They asked whether bounded-lowness and superlowness agree with each other on low c.e. sets. We will give a negative answer to this question by showing the existence of bounded-low c.e. sets which are low, but not superlow.

Theorem 2.1 There are low c.e. sets which are bounded-low but not superlow.

After seeing that superlow sets are always bounded-low, Anderson, Csima and Lange [2] asked whether any superhigh set is also bounded-high. Our second result in this chapter will provide a negative answer to this question. That is, we show the existence of bounded-low c.e. sets which are of degree $\mathbf{0}^{\prime}$. Thus, such bounded-low c.e. sets are superhigh.

Theorem $2.20^{\prime}$ contains a bounded-low c.e. set.

We will give a proof of Theorem 2.1 first, and then prove Theorem 2.2 in Section 2.4 .

To prove Theorem 2.1, we will construct a low c.e. set $A$ such that $A^{\dagger}$ is $\omega$-c.e. (so $A$ is bounded-low), and $A^{\prime}$ is not $\omega$-c.e. (so $A$ is not superlow). One approach 
of constructing a set low, but not superlow, is to apply Sacks splitting theorem, to split $\varnothing^{\prime}$ into two low c.e. sets $A$ and $B$. $A$ and $B$ cannot both be superlow, as if $A$ is superlow, then $\varnothing^{\prime}$ is $w t t$-reducible to $B$ [by a theorem of Bickford and Mills in [12]]. In same paper [12], Bickford and Mills also pointed out that there are superlow c.e. sets $A$ and $B$ such that $A \oplus B$ computes $\varnothing^{\prime}$. Most of the results in [12] can be found in Nies' book [27].

One feature of Sacks splitting is that there is no effective bound on the number of injuries from a strategy with higher priority, and hence we cannot have a recursive bound on the number of changes of $A^{\prime}(e)$ in advance. It also turns out to be an obstacle for us to obtain a bounded-low c.e. set which is low, but not superlow, by using Sacks splitting. Instead, we will construct a low, but not superlow, c.e. set directly.

\subsection{Requirements and strategies}

We will construct a c.e. set $A$ meeting the following requirements:

$\mathcal{L}_{e}$ : If there are infinitely many stages $s$ such that $\Phi_{e}^{A}(e)[s] \downarrow$, then $\Phi_{e}^{A}(e) \downarrow$.

$\mathcal{R}_{e}$ : The number of changes of $A^{\dagger}$ at $e$ is bounded by $2(e+1)^{2}$.

$\mathcal{P}_{\langle i, j\rangle}:$ If $\varphi_{i}$ and $\varphi_{j}^{2}$ are both total, then there is some $x$ such that either

$$
A^{\prime}(x) \neq \lim _{t \rightarrow \infty} \varphi_{j}^{2}(x, t)
$$

or

$$
\left|\left\{t \in \mathbb{N}: \varphi_{j}^{2}(x, t) \neq \varphi_{j}^{2}(x, t+1)\right\}\right| \geq \varphi_{i}(x) .
$$

Here, $\left\{\varphi_{i}: i \in \mathbb{N}\right\}$ and $\left\{\varphi_{j}^{2}: j \in \mathbb{N}\right\}$ are standard enumerations of all partial computable functions in one variable and two variables respectively, $\left\{\Phi_{e}: e \in \mathbb{N}\right\}$ is a standard enumeration of all partial computable functionals, and $\langle\cdot, \cdot\rangle$ is an effective bijection between $\mathbb{N}^{2}$ and $\mathbb{N}$.

We assign the priority of requirements in our finite injury construction as

$$
\mathcal{L}_{0} \prec \mathcal{R}_{0} \prec \mathcal{P}_{0} \prec \mathcal{L}_{1} \prec \mathcal{R}_{1} \prec \mathcal{P}_{1} \prec \cdots \prec \mathcal{L}_{e} \prec \mathcal{R}_{e} \prec \mathcal{P}_{e} \prec \cdots
$$


If all the $\mathcal{L}$-requirements are satisfied, then $A$ is low. If all the $\mathcal{R}$-requirements are satisfied, then the whole construction will ensure that $A^{\dagger}$ is $\omega$-c.e., which ensures that $A$ is bounded-low. If all the $\mathcal{P}$-requirements are satisfied, then $A^{\prime}$ is not $\omega$-c.e., and hence, $A$ is not superlow.

The strategy of satisfying one $\mathcal{L}_{e}$ is standard, i.e., we will set restraints to preserve the desired computations we have seen and this strategy can only be injured by $\mathcal{P}$ strategies with higher priority, which will happen at most finitely many times. Thus, after a stage large enough, after which no $\mathcal{P}$-strategies with higher priority act, if the strategy sets restraints to preserve computations, these computations will be preserved forever, and $\mathcal{L}_{e}$ is satisfied.

The strategy for satisfying one $\mathcal{R}_{e}$ is also to set restraints to preserve computations. At each stage $s$, when we see that $\varphi_{i}(e)$ converges, where $i \leq e$, we set a restraint to protect $A\left\lceil\varphi_{i}(e)\right.$. Note that $\mathcal{P}$-strategy with higher priority can injure $\mathcal{R}_{e}$ by enumerating small numbers $x$ into $A$. But as $\varphi_{i}(e)$ will be fixed, once $\mathcal{P}$ enumerates $x$ into $A, \mathcal{P}$ selects a new number, $x^{\prime}$ say, bigger than $\varphi_{i}(e)$, and the further enumeration of $x^{\prime}$ will not injure $\mathcal{R}_{e}$ [as this enumeration will not affect the computation $\Phi_{e}^{A\left\lceil_{\varphi_{i}(e)}\right.}(e)$ if $\Phi_{e}^{A\left\lceil_{\varphi_{i}(e)}\right.}(e)$ converges]. Note that there are at most $e+1$ many such restraints, and each $\mathcal{P}$-strategy with higher priority can enumerate numbers less than $\varphi_{i}(e)$ at most once, the total number of such enumerations is at most $(e+1)^{2}$. As one such an enumeration entails at most two changes of $A^{\dagger}(e)$, the number of changes of $A^{\dagger}(e)$ is bounded by $2(e+1)^{2}$.

We now describe how to satisfy a $\mathcal{P}_{\langle i, j\rangle}$-requirement. As in [27] for the construction of nonsuperlow sets, we will apply the recursion theorem for the construction. That is, our construction will be uniform in parameters, $r$ say, and in the $r$-th construction, we will build a partial computable functional $\Gamma_{r}$, a c.e. set of axioms $\langle\sigma, n, m\rangle$, where if $\langle\sigma, n, m\rangle$ is enumerated into $\Gamma_{r}$ at stage $s, \Gamma_{r}^{A}(n)[s]$ is defined as $m$ with use $A_{s}\left\lceil_{\gamma_{r}^{A}(n)[s]}=\sigma\right.$. From $\Gamma_{r}$, if $\Gamma_{r}=W_{r}$, we can have a computable function $p_{r}$ such that

$$
\forall X \forall x\left[\Gamma_{r}^{X}(x) \simeq \Phi_{p_{r}(x)}^{X}\left(p_{r}(x)\right)\right] .
$$

This will ensure that $\Gamma_{r}^{A}(x) \downarrow \Longleftrightarrow p_{r}(x) \in A^{\prime}$. Hence, by controlling $\Gamma_{r}^{A}$, we can defeat $\varphi_{j}^{2}$ being an approximation of $A^{\prime}$ with number of changes bounded by $\varphi_{i}$. 
Since the construction is uniform in $r$, there is a computable function $g$ such that $\Gamma_{r}=W_{g(r)}$. By recursion theorem, there is a $r_{0}$ such that $W_{g\left(r_{0}\right)}=W_{r_{0}}$, i.e., $\Gamma_{r_{0}}=W_{r_{0}}$. In the following, assume that we are in the $r_{0}$-th construction, just fix the computable function $p_{r_{0}}$ in advance.

A $\mathcal{P}_{\langle i, j\rangle}$-strategy proceeds as follows:

(i) Choose a fresh witness $x$, let $z=p_{r_{0}}(x)$.

(ii) Wait for $\varphi_{i}(z) \downarrow$ at some stage $s$.

(If we wait at (2) forever, then $\varphi_{i}(z) \uparrow$, and $\varphi_{i}$ is partial and $\mathcal{P}_{\langle i, j\rangle}$ is satisfied.)

(iii) Set $N=\varphi_{i}(z)+1$, and run the following modules $C(n)$ and $D(n)$ for $n \leq$ $N$. We start with $C(0)$ in which case $\varphi_{j}^{2}(z, 0) \downarrow=1$ or $D(0)$ in which case $\varphi_{j}^{2}(z, 0) \downarrow=0$, and let $\ell(-1)=0$. We will end when reaching $C(N)$ or $D(N)$, because this shows that $\varphi_{i}(z)$ cannot bound the number of changes of $\varphi_{j}^{2}(z, q)$, showing that $\mathcal{P}_{\langle i, j\rangle}$ is satisfied.

$C(n)$ : There is a $q_{1}>\ell(n-1)$ such that $\varphi_{j}^{2}$ converges (at stage $s$ ) to 1 on all $(z, q)$ with $\ell(n-1)<q \leq q_{1}$, and for those $q_{2}$ with $q_{1}<q_{2} \leq s$, if $\varphi_{j}^{2}\left(z, q_{2}\right)$ converges at stage $s$, then $\varphi_{j}^{2}\left(z, q_{2}\right)=1$.

If such a $q_{1}$ does not exist, then do nothing.

- If this is because of the fact that $\varphi_{j}^{2}(z, \ell(n-1)+1)$ never converges, then $\varphi_{j}^{2}$ is partial and $\mathcal{P}_{\langle i, j\rangle}$ is satisfied.

- If it is because of the existence of $q_{2}$ with $\varphi_{j}^{2}\left(z, q_{2}\right)=0$, then wait for a bigger stage at which $\varphi_{j}^{2}(z, q)$ converges for all $q \leq q_{2}$, leave $C(n)$ with no actions and switch to $D(n+1)$ with $\ell(n)=q_{2}$.

(Again, if $\varphi_{j}^{2}(z, q)$ never converges for some $q<q_{2}$, then $\varphi_{j}^{2}$ is partial and $\mathcal{P}_{\langle i, j\rangle}$ is satisfied.)

That is, at stage $s$, we guess that $\varphi_{j}^{2}(z, q)$ has limit 1 , and we should not keep such a guess if we see $\varphi_{j}^{2}(z, q)$ converges to 0 after $\ell(n-1)$.

Action for $C(n)$ : If $\Gamma_{r_{0}}^{A}(x)$ has definition at stage $s-1$, then enumerate $\gamma_{r_{0}}(x)$ into $A$, to undefine $\Gamma_{r_{0}}^{A}(x)$. Otherwise, do nothing. 
- Wait for a stage $t>s$ such that $\Phi_{z}^{A}(z)[t]$ diverges.

(For $r_{0}$-th construction, such a stage $t$ exists, because otherwise, we would have $\Gamma_{r_{0}}^{A}(x)$ diverges, but $\Phi_{z}^{A}(z)$ converges.)

- Wait for a bigger stage at which we see that $\varphi_{j}^{2}\left(z, q_{3}\right)$ converges to 0 , where $q_{3} \geq q_{1}$, and $\varphi_{j}^{2}(z, q)$ converges for all $q \leq q_{3}$. Let $\ell(n)=q_{3}$ and switch to $D(n+1)$.

(Again, if $\varphi_{j}^{2}(z, q)$ never converges for some $q<q_{3}$, then $\varphi_{j}^{2}$ is partial and $\mathcal{P}_{\langle i, j\rangle}$ is satisfied. Or, if $\varphi_{j}^{2}(z, q)$ converges to 1 for all $q \geq q_{1}$, then $\lim _{x \rightarrow \infty} \varphi_{j}^{2}(z, q)=1$, while $z \notin A^{\prime}$, meaning that $\lim _{x \rightarrow \infty} \varphi_{j}^{2}(z, q)$ is not the characteristic function of $A^{\prime}$, and $\mathcal{P}_{\langle i, j\rangle}$ is satisfied.)

$D(n)$ : There is a $q_{1}>\ell(n-1)$ such that $\varphi_{j}^{2}$ converges (at stage $s$ ) to 0 on all $(z, q)$ with $\ell(n-1)<q \leq q_{1}$, and for those $q_{2}$ with $q_{1}<q_{2} \leq s$, if $\varphi_{j}^{2}\left(z, q_{2}\right)$ converges at stage $s$, then $\varphi_{j}^{2}\left(z, q_{2}\right)=0$.

If there is no such a $q_{1}$, then do nothing.

- If this is because of the fact that $\varphi_{j}^{2}(z, \ell(n-1)+1)$ never converges, then $\varphi_{j}^{2}$ is partial and $\mathcal{P}_{\langle i, j\rangle}$ is satisfied.

- If it is because of the existence of $q_{2}$ with $\varphi_{j}^{2}\left(z, q_{2}\right)=1$, then wait for a bigger stage at which $\varphi_{j}^{2}(z, q)$ converges for all $q \leq q_{2}$, leave $D(n)$ with no actions and switch to $C(n+1)$ with $\ell(n)=q_{2}$. (Again, if $\varphi_{j}^{2}(z, q)$ never converges for some $q<q_{2}$, then $\varphi_{j}^{2}$ is partial and $\mathcal{P}_{\langle i, j\rangle}$ is satisfied.)

That is, at stage $s$, we guess that $\varphi_{j}^{2}(z, q)$ has limit 0, and we should not keep such a guess if we see $\varphi_{j}^{2}(z, q)$ converges to 1 after $\ell(n-1)$.

Action for $D(n)$ : If $\Gamma_{r_{0}}^{A}(x)$ has no definition at stage $s-1$, define $\Gamma_{r_{0}}^{A}(x)$ with use $\gamma_{r_{0}}(x)$ big.

- Wait for a stage $t>s$ such that $\Phi_{z}^{A}(z)[t]$ converges.

(For $r_{0}$-th construction, such a stage $t$ exists, because otherwise, we would have $\Gamma_{r_{0}}^{A}(x)$ converges, but $\Phi_{z}^{A}(z)$ diverges.) 
- Wait for a bigger stage at which we see that $\varphi_{j}^{2}\left(z, q_{3}\right)$ converges to 1 , where $q_{3} \geq q_{1}$, and $\varphi_{j}^{2}(z, q)$ converges for all $q \leq q_{3}$. Let $\ell(n)=q_{3}$ and switch to $C(n+1)$.

(Again, if $\varphi_{j}^{2}(z, q)$ never converges for some $q<q_{3}$, then $\varphi_{j}^{2}$ is partial and $\mathcal{P}_{\langle i, j\rangle}$ is satisfied. Or, if $\varphi_{j}^{2}(z, q)$ converges to 0 for all $q \geq q_{1}$, then $\lim _{x \rightarrow \infty} \varphi_{j}^{2}(z, q)=0$, while $z \in A^{\prime}$, meaning that $\lim _{x \rightarrow \infty} \varphi_{j}^{2}(z, q)$ is not the characteristic function of $A^{\prime}$, and $\mathcal{P}_{\langle i, j\rangle}$ is satisfied.)

Thus, a $\mathcal{P}_{\langle i, j\rangle}$-strategy either waits at some $C(n)$ or $D(n)$ for some $n<N$, or reaches $C(N)$ or $D(N)$, both of which will show that $\mathcal{P}_{\langle i, j\rangle}$ is satisfied, as explained above. Thus, in the construction, a $\mathcal{P}_{\langle i, j\rangle}$-strategy can injure those strategies with lower priority at most finitely often.

\subsection{Construction}

\section{Construction of $A$.}

Stage 0: Let $A_{0}=\varnothing$, and initialize all strategies.

Stage $s>0$ : Find a requirement with the highest priority, $Q$ say, among

$$
\mathcal{L}_{0} \prec \mathcal{R}_{0} \prec \mathcal{P}_{0} \prec \cdots \mathcal{L}_{s} \prec \mathcal{R}_{s} \prec \mathcal{P}_{s}
$$

that requires attention at stage $s$, and act accordingly.

Say that $\mathcal{L}_{e}$ requires attention at stage $s$ if $\Phi_{e}^{A}(e)[s-1] \uparrow$ and $\Phi_{e}^{A}(e)[s] \downarrow$. Say $\mathcal{R}_{e}$ requires attention at stage $s$ if there is a $n \leq e \leq s$ such that $\varphi_{n}(e) \downarrow$ at stage $s$ and $\mathcal{R}_{e}$ has not set $A$-restraint $\varphi_{n}(e)$ yet.

For both cases, we act as follows:

Action: Set $A$-restraint as $s$, and initialize all strategies with lower priority.

Say that $\mathcal{P}_{\langle i, j\rangle}$ requires attention at stage $s$ if one of the following applies:

(i) $\mathcal{P}_{\langle i, j\rangle}$ has no witness currently.

Action: Pick a fresh number $x$ as a witness for $\mathcal{P}_{\langle i, j\rangle}$. 
(ii) $x$ is selected, $p_{r_{0}}(x)$ converges to $z, \varphi_{i}(z)[s] \downarrow$ and $N$ is not defined yet.

Action: Definite $N$ as $\varphi_{i}(z)+1$.

(iii) $N$ is defined, $\mathcal{P}_{\langle i, j\rangle}$ is not in $C(n)$ or $D(n)$ for any $n \leq N$, and $\varphi_{j}^{2}(z, 0)[s]$ converges.

Action: Let $\mathcal{P}_{\langle i, j\rangle}$ start by running $C(0)$, if $\varphi_{j}^{2}(z, 0)[s] \downarrow=1$, or $D(0)$, if $\varphi_{j}^{2}(z, 0)[s] \downarrow=0$. Here we let $\ell(-1)=0$.

(iv) $\mathcal{P}_{\langle i, j\rangle}$ switches from $C(n)$ to $D(n+1)$, where $n+1<N$.

Action: If $\Gamma^{A}(x)$ has no definition at stage $s-1$, then define $\Gamma^{A}(x)[s]=0$ with use $\gamma(x)[s]$ larger than all numbers used before.

(v) $\mathcal{P}_{\langle i, j\rangle}$ switches from $D(n)$ to $C(n+1)$, where $n+1<N$.

Action: If $\Gamma^{A}(x)$ has definition at stage $s-1$, then enumerate $\gamma(x)$ into $A$, undefining $\Gamma^{A}(x)$.

(vi) $\mathcal{P}_{\langle i, j\rangle}$ switches from $C(N-1)$ to $D(N)$ or from $D(N-1)$ to $C(N)$.

Action: Declare that $\varphi_{i}(z)$ cannot bound the number of changes of $\varphi_{j}^{2}(z, q)$, and that $\mathcal{P}_{\langle i, j\rangle}$ is satisfied.

If $\mathcal{P}_{\langle i, j\rangle}$ acts as above, then all strategies with lower priority are initialized.

This ends the construction of $A$ at stage $s$.

\subsection{Verification}

Lemma 2.1 For each requirement, $Q$ say,

(1) $Q$ can be initialized at most finitely often;

(2) $Q$ acts at most finitely often and is satisfied;

(3) The restraint set by $Q$ is finite, and $Q$ can initialize all strategies with lower priority finitely often. 
Proof: We will show that for each $e$, (1)-(3) are true for all $\mathcal{L}_{e}, \mathcal{R}_{e}, \mathcal{P}_{\langle i, j\rangle}$, where $\langle i, j\rangle=e$. We prove it by induction.

When $e=0, \mathcal{L}_{0}$ can never be initialized, due to its highest priority. (1) holds. $\mathcal{L}_{0}$ acts and sets a restraint only when $\Phi_{0}^{A}(0)$ converges, and once we set such a restraint, $\Phi_{0}^{A}(0)$ converges at any further stage, and (2) holds. In the construction, $\mathcal{L}_{0}$ sets a restraint only when $\Phi_{0}^{A}(0)$ converges, and after it acts, it will never act again. (3) holds.

Note that $\mathcal{R}_{0}$ can be initialized by $\mathcal{L}_{0}$ at most once. (1) holds for $\mathcal{R}_{0}$. Note that $\mathcal{R}_{0}$ acts only when $\varphi_{0}(0)$ converges, and after this, if $\Phi_{0}^{A\left\lceil\varphi_{0}(0)\right.}(0)$ converges, then it will converge forever, as no $\mathcal{P}$ can enumerate numbers less than $\varphi_{0}(0)$ into $A$. So if $\Phi_{0}^{A\left\lceil\varphi_{0}(0)\right.}(0)$ converges again, then it converges forever, which means that $\mathcal{R}_{0}$ is satisfied. (2) holds. The restraint set by $\mathcal{R}_{0}$ is $\varphi_{0}(0)$, if it converges, and after it acts, it will never act again. (3) holds.

For $\mathcal{P}_{\langle 0,0\rangle}$, it can be initialized by $\mathcal{L}_{0}$ and $\mathcal{R}_{0}$, so $\mathcal{P}_{\langle 0,0\rangle}$ can be initialized by $\mathcal{L}_{0}$ and $\mathcal{R}_{0}$ at most three times. (1) holds. Let $s_{0}$ be the least stage after which $\mathcal{P}_{\langle 0,0\rangle}$ can not be initialized again, and suppose that $\mathcal{P}_{\langle 0,0\rangle}$ selects a witness $x$ as a big number at stage $s_{1}>s_{0}$. Then $x$ cannot be canceled later. Then after stage $s_{1}$, $\mathcal{P}_{\langle 0,0\rangle}$ acts only when

(i) it selects $N$; or

(ii) it starts module $C(0)$ or $D(0)$; or

(iii) it switches from $C(n-1)$ to $D(n)$, or from $D(n-1)$ to $C(n)$; or

(iv) it reaches $C(N)$ or $D(N)$,

which can happen at most $N+2$ many times. If it reaches $C(N)$ or $D(N)$, then $\varphi_{0}(z)$ cannot be a bound of the number of changes of $\varphi_{0}^{2}(z, q)$, and $\mathcal{P}_{\langle 0,0\rangle}$ is satisfied. If it never selects $N$, then $\varphi_{0}(z)$ never converges (recall that $p_{r_{0}}$ is assumed to be total, by the relativized $s_{n}^{m}$-theorem). Here $z=p_{r_{0}}(x)$. If it never starts module $C(0)$ or $D(0)$, then $\varphi_{0}^{2}(z, 0)$ diverges. If it stays at some module $C(n)$ or $D(n)$ forever, then either $\varphi_{0}^{2}(z, q)$ diverges for some $q$, or we will have $\lim _{q \rightarrow \infty} \varphi_{0}^{2}(z, q) \neq A^{\prime}(z)$. $\mathcal{P}_{\langle 0,0\rangle}$ is satisfied for all cases. (2) holds for $\mathcal{P}_{\langle 0,0\rangle}$. Thus, after a stage $s_{3}$ large enough, $\mathcal{P}_{\langle 0,0\rangle}$ 
will have no actions, and hence the restraint set by $\mathcal{P}_{\langle 0,0\rangle}$ is finite, and it will not initialize strategies with lower priority. (3) holds.

For $e>0$, we assume that the lemma is true for $e^{\prime}<e$. The proof that the lemma is true for $e$ is very similar to the proof for the basic case, i.e. $e=0$, and we will assume that after a stage $s$ big enough, no strategies $\mathcal{L}_{e^{\prime}}, \mathcal{R}_{e^{\prime}}$, and $\mathcal{P}_{e^{\prime}}$ can act. Then we can show that after stage $s, \mathcal{L}_{e}, \mathcal{R}_{e}$, and $\mathcal{P}_{e}$ will behave exactly like $\mathcal{L}_{0}, \mathcal{R}_{0}$, and $\mathcal{P}_{0}$ above, and hence the lemma is true for $e$.

This completes the proof of Lemma 2.1.

By Lemma 2.1, $A$ is low but not superlow. Lemma 2.2 below shows that $A$ is bounded-low.

Lemma 2.2 $A^{\dagger}$ is w-c.e., and hence $A$ is bounded-low.

Proof: To show that $A^{\dagger}$ is $\omega$-c.e., we need to show that there is a computable function bounding the changes of $e$ in $A^{\dagger}$ for all $e$.

Fix $e$, and $n \leq e$. Then in the construction, whenever we see $\varphi_{n}(e)$ converges, at stage $s$ say, $\mathcal{R}_{e}$ acts and initializes all strategies with lower priority. Thus, no strategy with lower priority can put a number less that $\varphi_{n}(e)$ into $A$ later. So if

$\Phi_{e}^{A\left\lceil\varphi_{n}(e)\right.}$ converges later ( $e$ enters $A^{\dagger}$ ), this computation can only be changed by $\mathcal{P}_{e^{\prime-}}$ strategies (where $e^{\prime}<e$, which have higher priority) by enumerating $\gamma_{r_{0}}^{A}(y)[t]$ into $A$. After this, if $\gamma_{r_{0}}^{A}(y)$ is defined later, then it will be defined as a number bigger than $\varphi_{n}(e)$. Thus, because of this, $\Phi_{e}^{A\left\lceil\varphi_{n}(e)\right.}$ is injured at most $e+1$ many times, then $A^{\dagger}(e)$ can change at most $2(e+1)$ many times, for this particular $n$. Thus, in total, $A^{\dagger}(e)$ can change at most $2(e+1)^{2}$ many times, and $A^{\dagger}$ is $\omega$-c.e.

This completes the proof of Theorem 2.1.

\subsection{A bounded-low set with Turing degree $0^{\prime}$}

In this section, we give a sketchy proof of Theorem 2.2. That is, we will construct a bounded-low c.e. set $A$ Turing computing $\varnothing^{\prime}$. We have seen how to construct a bounded-low c.e. set in the proof of Theorem 2.1, so to prove Theorem 2.2, we only need to show how to make $A$ Turing complete. We will construct a partial computable functional $\Gamma$ such that $\varnothing^{\prime}=\Gamma^{A}$. Of course, the construction of $\Gamma$ will be consistent with the $\mathcal{R}$-strategies of making $A$ bounded-low. 
For the construction of $\Gamma$, we will make sure that for each $e, \Gamma^{A}(e)$ is defined and computes $\varnothing^{\prime}(e)$ correctly. The idea of constructing $\Gamma$ is quite standard. That is, in the construction, at stage $s$, we find the least $x$ with $\Gamma^{A}(x)$ not defined and define $\Gamma^{A}(x)[s]=\varnothing^{\prime}(x)[s]$ with use $\gamma(x)$ big, like $s$. If later, at stage $t>s, x$ enters $\varnothing^{\prime}$, then at this stage, we enumerate $\gamma(x)$ into $A$, undefining $\Gamma^{A}(x)$. In general, the construction of $\Gamma$ satisfies the following rules:

(i) For any $x_{1}, x_{2}$ with $x_{1}<x_{2}$, if $\Gamma^{A}\left(x_{2}\right)$ has definition at stage $s$, then $\Gamma^{A}\left(x_{1}\right)$ also has definition at this stage, with uses $\gamma\left(x_{1}\right)<\gamma\left(x_{2}\right)$.

(ii) For any $x_{1}, x_{2}$ with $x_{1}<x_{2}$, if $\Gamma^{A}\left(x_{1}\right)$ is undefined at stage $s$, then $\Gamma^{A}\left(x_{2}\right)$ is also undefined at this stage, due to a number less than $\gamma\left(x_{1}\right)$ enters $A$.

(iii) For any $x$, if $\Gamma^{A}(x)$ has definition at stage $s_{1}$ and $s_{2}$, then $\gamma(x)\left[s_{1}\right] \leq \gamma(x)\left[s_{2}\right]$, and if $\gamma(x)\left[s_{1}\right]<\gamma(x)\left[s_{2}\right], \Gamma^{A}(x)$ must have been undefined between these two stages.

(iv) For any $x, \Gamma^{A}(x)$ can be undefined at most finitely often during the construction.

(v) For any $x, \Gamma^{A}(x)=\varnothing^{\prime}(x)$.

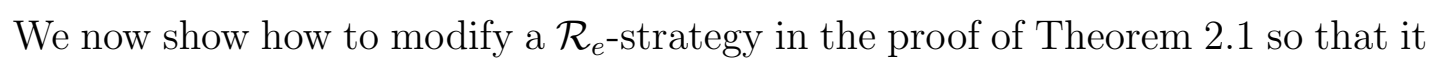
is consistent with the rules above. Recall that the $\mathcal{R}_{e}$-strategy can act finitely many times, and each time when it acts, because of $\varphi_{i}(e) \downarrow$ for some $i \leq e$, it initializes all strategies with lower priority. To make it consistent with the construction of $\Gamma$, it needs to have an additional action: enumerate $\gamma(e)[s]$ into $A$. This enumeration undefines $\Gamma^{A}(e)$, so when it is defined again, the use will be defined as a big number, which is bigger than the value $\varphi_{i}(e)$. Thus, for a fixed $e$, the membership of $e$ in

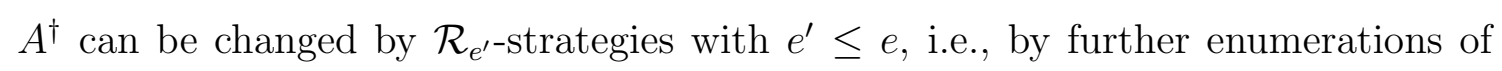
the $\gamma$-uses, or by rectifying $\Gamma^{A}(x)=1$ when some $x<e$ enumerating into $\varnothing^{\prime}$. Then $A^{\dagger}(e)$ changes at most $2(e+1)^{2}$ many times, which guarantees that $A^{\dagger}$ is $\omega$-c.e.

The construction and verification parts are standard finite injury arguments. 


\section{Chapter 3}

\section{A bounded-high set which is high, but not superhigh}

Note that $\varnothing^{\prime}$ is a bounded-high set which is also superhigh. In [2], Anderson, Csima and Lange further asked whether there are bounded-high sets which are high but not superhigh. Similarly, based on the existence of superhigh bounded-low sets, we can also ask whether there are high but not superhigh bounded-low sets. In sections 3.1 and 3.2, we will provide two pseudo-jump inversion theorems for bounded-high sets and bounded-low sets, which will provide positive answers to these questions.

Let $W_{e}$ be a c.e. operator, the corresponding pseudo-jump operator $V_{e}$ is defined as $V_{e}^{A}=A \oplus W_{e}^{A}$ for any set $A$. One way to obtain high, but not superhigh, c.e. sets is through the pseudo-jump inversion theorem of Jockusch and Shore [28] which says: for any c.e. operator $W_{e}$, there is a c.e. set $C$ such that $V_{e}^{C} \equiv_{T} \varnothing^{\prime}$. This idea was first used by Mohrherr in her paper [4].

The procedure to obtain high but not superhigh c.e. sets are:

(1) Through the construction of low but not superlow c.e. sets, one can obtain a c.e. operator $W$ such that for any set $A, A \oplus W^{A}$ is low over $A$, but not superlow over $A$, i.e., $\left(A \oplus W^{A}\right)^{\prime} \leq_{T} A^{\prime}$, but $\left(A \oplus W^{A}\right)^{\prime} \underline{\leq}_{t t} A^{\prime}$.

(2) By applying the pseudo-jump inversion theorem to this operator $W$, there exists a c.e. set $C$ such that $C \oplus W^{C} \equiv_{T} \varnothing^{\prime}$.

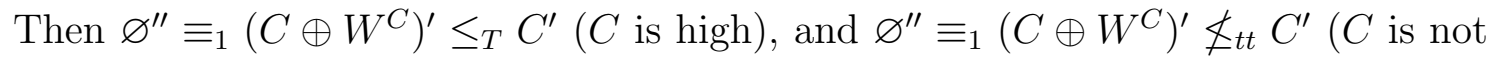
superhigh). 
In this chapter, we will provide two strengthened versions of Jockusch and Shore's pseudo-jump inversion theorem. That is, $C$ in Jockusch and Shore's theorem can be bounded-high (section 3.1) or bounded-low (section 3.2).

Theorem 3.1 (1) For any c.e. operator $W$, there is a bounded-high set $C$ such that $C \oplus W^{C} \equiv_{T} \varnothing^{\prime}$.

(2) For any c.e. operator $W$, there is a bounded-low c.e. set $C$ such that $C \oplus$ $W^{C} \equiv_{T} \varnothing^{\prime}$.

The following two results follow Theorem 3.1 immediately:

Corollary 3.1 (1) There are bounded-high sets which are high but not superhigh.

(2) There are bounded-low c.e. sets which are high but not superhigh.

Proof: $\quad$ Let $W$ be the c.e. operator such that for any set $A, A \oplus W^{A}$ is low but not superlow over $A$, then any $C$ satisfying $C \oplus W^{C} \equiv_{T} \varnothing^{\prime}$ is high but not superhigh. By Theorem 3.1, there are a bounded-high set $C_{1}$ and a bounded-low c.e. set $C_{2}$ such that $C_{1} \oplus W^{C_{1}} \equiv_{T} \varnothing^{\prime}$ and $C_{2} \oplus W^{C_{2}} \equiv_{T} \varnothing^{\prime}$. Thus $C_{1}$ is a bounded-high set which is high but not superhigh, while $C_{2}$ is a bounded-low c.e. set which is high but not superhigh.

We will prove Theorem 3.1.(1) in Section 3.1, and then prove Theorem 3.1.(2) in Section 3.2.

For a c.e. operator $W$, let $\Psi$ be the corresponding partial computable functional. That is, for any oracle $A, e \in W^{A}[s] \Leftrightarrow \Psi^{A}(e)[s] \downarrow$. We view the use $\psi^{A}(e)[s]$ of $\Psi^{A}(e)[s]$ as the use for $e \in W^{A}[s]$. That is, if $A \uparrow_{\psi^{A}(e)[s]}$ does not change later, $e$ will stay in $W^{A}$. $\Psi$-uses satisfy the following usual use rules: if $\Psi^{A}(e)[s] \downarrow$ and $\Psi^{A}\left(e^{\prime}\right)[s] \downarrow$ with $e<e^{\prime}$, then $\psi^{A}(e)[s]<\psi^{A}\left(e^{\prime}\right)[s]$; if $\Psi^{A}(e)[s] \downarrow$, then $\psi^{A}(e)[s] \leq s$; and if $\Psi^{A}(e)[s] \uparrow$, then $\psi^{A}(e)[s]=0$.

\subsection{Pseudo-jump inversion via bounded-high sets}

We will construct a $\omega$-c.e. set $C$ (so $C \leq_{w t t} \varnothing^{\dagger}$ ) such that $C \oplus W^{C} \equiv_{T} \varnothing^{\prime}$ and $\varnothing^{\dagger \dagger} \leq_{w t t} C^{\dagger}$. $C$ will meet the following requirements: 
$\mathcal{P}_{e}: \varnothing^{\prime}(e)=\Gamma^{C \oplus W^{C}}(e)$, where $\Gamma$ is a computable functional built by us.

$\mathcal{Q}_{e}: \varnothing^{\dagger \dagger}(e)=\left.\Delta^{C^{\dagger}}\right|_{g(e)}(e)$, where $\Delta$ is a computable functional built by us and $g$ is a computable function such that the use $\delta^{C^{\dagger}}(e) \leq g(e)$ for all $e$.

$\mathcal{R}_{e}$ : If there are infinitely many stages $s$ with $e \in W^{C}[s]$, then $e \in W^{C}$.

If all the $\mathcal{R}$-requirements are satisfied, then $W^{C}$ is $\Delta_{2}^{0}$. By construction, $C$ will be $\omega$-c.e., and hence $C \oplus W^{C} \leq_{T} \varnothing^{\prime}$. If all the $\mathcal{P}$-requirements are satisfied, then $\varnothing^{\prime} \leq_{T} C \oplus W^{C}$. Thus, $\varnothing^{\prime} \equiv_{T} C \oplus W^{C}$. If all the $\mathcal{Q}$-requirements are satisfied, then $\varnothing^{\dagger \dagger} \leq{ }_{w t t} C^{\dagger}$, and $C$ will be bounded-high.

We will apply the bounded-high strategy developed by Anderson, Csima and Lange in [2], and combine it with Jockusch and Shore's pseudo-jump inversion theorem. Our construction is a finite injury argument, where the priority of requirements is

$$
\mathcal{R}_{0} \prec \mathcal{P}_{0} \prec \mathcal{Q}_{0} \prec \mathcal{R}_{1} \prec \mathcal{P}_{1} \prec \mathcal{Q}_{1} \prec \cdots \mathcal{R}_{e} \prec \mathcal{P}_{e} \prec \mathcal{Q}_{e} \prec \cdots
$$

\subsubsection{A $\mathcal{R}_{e^{- \text {strategy }}}$}

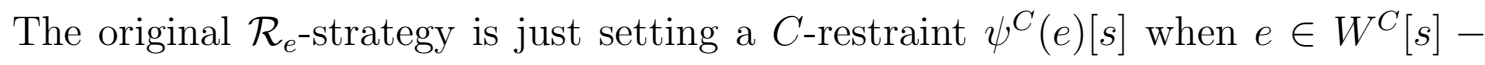
$W^{C}[s-1]$ (i.e., $\Psi^{C}(e)[s] \downarrow$ and $\left.\Psi^{C}(e)[s-1] \uparrow\right)$.

We first define $C$-restraint

$$
r(e, s)=\max \left\{\psi^{C}(e)\left[s^{\prime}\right]: s^{\prime} \leq s \wedge \Psi^{C}(e)\left[s^{\prime}\right] \downarrow\right\}
$$

Then $r(e, s) \leq r(e, s+1)$. Suppose that $e \in W^{C}[s]-W^{C}[s-1]$. e will be removed out of $W^{C}[t]$ at some least stage $t>s$ only if

$$
C_{t} \uparrow_{\psi^{C}(e)[s]} \neq C_{s} \uparrow_{\psi^{C}(e)[s]}
$$

So if $e \in W^{C}[s]$ and $C_{s} \uparrow_{r(e, s)}=C_{t} \uparrow_{r(e, s)}$ for all $t \geq s$, then $e \in W^{C}[t]$ for all stages $t \geq s$, and thus $e \in W^{C}$.

We say that $\mathcal{R}_{e}$ is injured at stage $s$ if either some $\mathcal{P}_{i}$ enumerates the $\Gamma$-use $\gamma^{C \oplus W^{C}}(i)[s] \leq r(e, s)$ into $C$ when $i \in \varnothing^{\prime}[s]-\varnothing^{\prime}[s-1]$; or some $\mathcal{Q}_{i}$ enumerates its coding indicator $c \leq r(e, s)$ into $C$ or extracts its coding indicator $c \leq r(e, s)$ out of $C$ when redefining $\Delta^{C^{\dagger} \uparrow_{g(i)}}(i)[s]=\varnothing^{\dagger \dagger}[s](i)$ (the definition of coding indicators will be clarified in the following basic $\mathcal{Q}$-strategy). 
During the construction, we do not allow positive requirements with lower priority, namely $\mathcal{P}_{j}, \mathcal{Q}_{j}$ with $j \geq e$, to enumerate elements $\leq r(e, s)$ into $C$ or to extract elements $\leq r(e, s)$ out of $C$, at any stage $s$ (this will be clear in Case 1 for $\mathcal{P}_{j}$ and Case 2 for $\mathcal{Q}_{j}$ ), and hence these requirements cannot injure $\mathcal{R}_{e}$ later.

Assume that $e \in W^{C}[s]-W^{C}[s-1]$ with $C$-restraint $r(e, s)$. Since we have appointed a $C$-restraint $r(e, s-1)$, we only need to consider the case $r(e, s)>$ $r(e, s-1)$. So we assume that at stage $s, r(e, s)>r(e, s-1)$.

Case 1. At stage $s$ with $r(e, s)>r(e, s-1)$, for $\mathcal{P}_{j}$ with $j \geq e$, we will show that $\Gamma^{C \oplus W^{C}}(e)[s] \uparrow$. That is, for all stages $s^{\prime}<s$ at which $\Gamma^{C \oplus W^{C}}(e)\left[s^{\prime}\right] \downarrow$, either

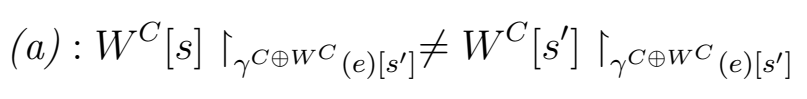

or

$$
\text { (b): } \quad C_{s} \uparrow_{\gamma C \oplus W^{C}(e)\left[s^{\prime}\right]} \neq C_{s^{\prime}} \uparrow_{\gamma}^{C \oplus W^{C}(e)\left[s^{\prime}\right]}
$$

holds.

Indeed, for stages $s^{\prime}<s$, if $\Gamma^{C \oplus W^{C}}(e)\left[s^{\prime}\right] \downarrow$ with $\Psi^{C}(e)\left[s^{\prime}\right] \uparrow$, then $e \notin W^{C}\left[s^{\prime}\right]$. As $e \in W^{C}[s]$,

$$
W^{C}[s] \uparrow_{e} \neq W^{C}\left[s^{\prime}\right] \Upsilon_{e}
$$

(recall that the symbol $X \uparrow_{z}$ denotes the set $\{x \in X: x \leq z\}$ ). As we will choose $\gamma^{C \oplus W^{C}}(e)\left[s^{\prime}\right]>e,(a)$ holds.

If $\Gamma^{C \oplus W^{C}}(e)\left[s^{\prime}\right] \downarrow$ with $\Psi^{C}(e)\left[s^{\prime}\right] \downarrow$. By the assumption that $e \in W^{C}[s]-W^{C}[s-1]$ with new computation $\Psi^{C}(e)[s] \downarrow$, we have

$$
C_{s} \Upsilon_{\psi^{C}(e)\left[s^{\prime}\right]} \neq C_{s^{\prime}} \Upsilon_{\psi^{C}(e)\left[s^{\prime}\right]} .
$$

Claim $\gamma^{C \oplus W^{C}}(e)\left[s^{\prime}\right]>\psi^{C}(e)\left[s^{\prime}\right]$, then $(b)$ holds.

We now prove

$$
\gamma^{C \oplus W^{C}}(e)\left[s^{\prime}\right]>\psi^{C}(e)\left[s^{\prime}\right]
$$

for all $s^{\prime}<s$ with $\Gamma^{C \oplus W^{C}}(e)\left[s^{\prime}\right] \downarrow$ and $\Psi^{C}(e)\left[s^{\prime}\right] \downarrow$. Suppose that the computation $\Gamma^{C \oplus W^{C}}(e)\left[s^{\prime}\right]$ is first defined at stage $t^{\prime} \leq s^{\prime}$ and returns back at stage $s^{\prime}$. This means that

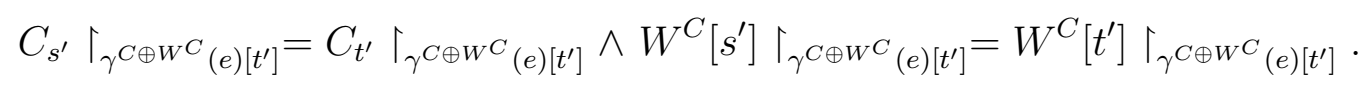

There are two cases: 
(1) $\Psi^{C}(e)\left[t^{\prime}\right] \downarrow$. As $\gamma^{C \oplus W^{C}}(e)\left[t^{\prime}\right]$ is defined to be bigger than all used numbers,

$$
\gamma^{C \oplus W^{C}}(e)\left[t^{\prime}\right]>\psi^{C}(e)\left[t^{\prime}\right]
$$

Then $\Psi^{C}(e)\left[t^{\prime}\right]$ also comes back at stage $s^{\prime}$, we have

$$
\gamma^{C \oplus W^{C}}(e)\left[s^{\prime}\right]=\gamma^{C \oplus W^{C}}(e)\left[t^{\prime}\right]>\psi^{C}(e)\left[t^{\prime}\right]=\psi^{C}(e)\left[s^{\prime}\right]
$$

(2) $\Psi^{C}(e)\left[t^{\prime}\right] \uparrow$. Then $e \notin W^{C}\left[t^{\prime}\right]$, but by assumption, $e \in W^{C}\left[s^{\prime}\right]$. As $\gamma^{C \oplus W^{C}}(e)\left[t^{\prime}\right]>$ $e$

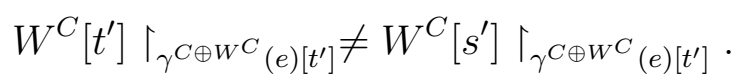

This contradicts with the assumption that the old computation $\Gamma^{C \oplus W^{C}}(e)\left[t^{\prime}\right] \downarrow$ returns back at stage $s^{\prime}$, so this case is indeed impossible.

In construction, at each stage $t$, if $\Gamma^{C \oplus W^{C}}(e)[t] \downarrow$ and $\Gamma^{C \oplus W^{C}}(j)[t] \downarrow$ with $e<j$, we will make the corresponding uses satisfying $\gamma^{C \oplus W^{C}}(e)[t]<\gamma^{C \oplus W^{C}}(j)[t]$. So when $\Gamma^{C \oplus W^{C}}(e)[s]$ is undefined, then all $\Gamma^{C \oplus W^{C}}(j)$ with $j>e$ are also undefined at stage $s$.

Hence, when the $C$-restraint $r(e, s)$ moves to a higher level, all the previous $\Gamma^{C \oplus W^{C}}(j)$-computations with $j \geq e$ are undefined, we will define new computations $\Gamma^{C \oplus W^{C}}(j)$ with use $>r(e, s)$. Then the enumerations of such uses into $C$ can not injure $\mathcal{R}_{e}$ as long as $r(e, s)$ does not increase later.

Case 2. At stage $s$ with $r(e, s)>r(e, s-1)$, for $\mathcal{Q}_{j}$ with $j \geq e$, we first consider $\mathcal{Q}_{e}$. Say that $\mathcal{Q}_{e}$ is injured at stage $s$ if there is an $i \leq e$ such that the $C$-restraint $r(i, s)$ on $\mathcal{R}_{i}$ is larger than or equal to the coding indicator of $\mathcal{Q}_{e}$.

The strategy of $\mathcal{Q}_{e}$ to deal with this injury from $\mathcal{R}_{i}$ is still to undefine previ-

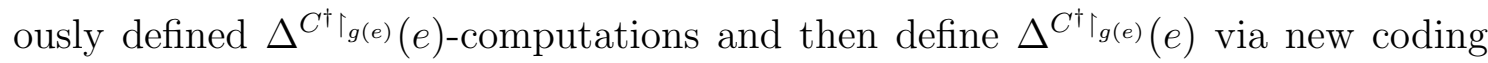
indicators $>r(i, s)$. Then $\mathcal{Q}_{e}$ will not injure $\mathcal{R}_{i}$ as long as $r(i, s)$ does not increase later.

To undefine $\Delta^{C^{\dagger} g_{(e)}}(e)$-computations, we need to change $C^{\dagger}$ below $g(e)$. In order to enumerate elements into or extract elements out of $C^{\dagger}$, we will build a partial computable function $q$ and a partial computable functional $\Theta$, and assume that the recursion theorem provides us the graph of $q$ and the c.e. relation of $\Theta$. 
Then by controlling $q$ and $\Theta$, we can move elements (i.e., indices of $\Theta$ obtained by $s_{n}^{m}$-theorem) into or out of $C^{\dagger}$ (this will be much clear in the following basic $\mathcal{Q}_{e}$-strategy).

Hence, during the construction, $\mathcal{R}_{e}$ can only be injured by positive requirements with higher priority. Each $\mathcal{P}_{i}(i<e)$ can injure $\mathcal{R}_{e}$ at most once, and each $\mathcal{Q}_{i}(i<e)$ can injure $\mathcal{R}_{e}$ finitely often by enumerating coding indicators. So $\mathcal{R}_{e}$ can be injured finitely often, and thus $\lim _{s} r(e, s)<\infty$.

\subsubsection{A $\mathcal{P}_{e^{- \text {strategy }}}$}

We will build a functional $\Gamma$ such that $\varnothing^{\prime}=\Gamma^{C \oplus W^{C}}$. At each stage $s$, when $e \in$ $\varnothing^{\prime}[s+1]-\varnothing^{\prime}[s]$ and $\Gamma^{C \oplus W^{C}}(e)[s] \downarrow$, we will enumerate the use $\gamma^{C \oplus W^{C}}(e)[s]$ into $C$ to undefine $\Gamma^{C \oplus W^{C}}(e)$, and then redefine $\Gamma^{C \oplus W^{C}}(e)=1$ with big use.

When the $C$-restraints $r(i, s+1)$ on $\mathcal{R}_{i}$ with $i \leq e$ move up, i.e. $r(i, s+1)>$

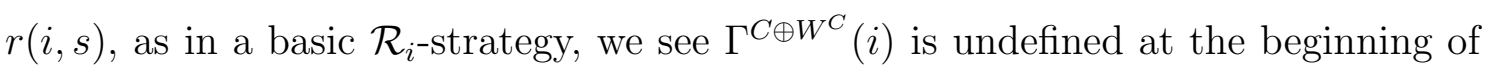
stage $s+1$. Thus, $\Gamma^{C \oplus W^{C}}(j) \uparrow$ for all $j \geq i$, and such $\Gamma^{C \oplus W^{C}}(j)$ will be defined later with new use $>r(i, s+1)$.

During the construction, we will define at most one $\Gamma^{C \oplus W^{C}}(y)$ at each stage. At the last step of stage $s+1$, we find the least $y$ with $\Gamma^{C \oplus W^{C}}(y) \uparrow$ currently.

If $\Gamma^{C \oplus W^{C}}(y)$ is never defined before, or there is a largest stage $w<s+1$ at which $\Gamma^{C \oplus W^{C}}(y)[w] \downarrow$ and also at least one of the following three conditions holds:

(1) $y \in \varnothing^{\prime}[w+1]-\varnothing^{\prime}[w]$;

(2) $y \in W^{C}[t+1]-W^{C}[t]$ with $r(y, t+1)>r(y, t)$ for some $t \in[w, s]$;

(3) $\gamma^{C \oplus W^{C}}(y)[w] \leq \gamma^{C \oplus W^{C}}(y-1)[s]$.

Then define $\Gamma^{C \oplus W^{C}}(y)[s+1]=\varnothing^{\prime}[s+1](y)$ with use $\gamma^{C \oplus W^{C}}(y)[s+1]$ bigger than all numbers used before.

Otherwise, there is a largest stage $w<s+1$ at which $\Gamma^{C \oplus W^{C}}(y)[w] \downarrow$ and none of three conditions above holds, just define $\Gamma^{C \oplus W^{C}}(y)[s+1]=\varnothing^{\prime}[s+1](y)$ with use $\gamma^{C \oplus W^{C}}(y)[s+1]=\gamma^{C \oplus W^{C}}(y)[w]$.

We now show that $\lim _{s} \gamma^{C \oplus W^{C}}(e)[s]<\infty$. Assume that $\lim _{s} \gamma^{C \oplus W^{C}}(e-1)[s]<\infty$, and that $\lim _{s} r(i, s)<\infty$ for all $i \leq e$. We can find a stage $s_{0}$ such that for all $i \leq e$, 
- $\lim _{s} r(i, s)=r\left(i, s_{0}\right)$,

- $i \in \varnothing^{\prime} \Leftrightarrow i \in \varnothing^{\prime}\left[s_{0}\right]$.

Let $s_{1} \geq s_{0}+1$ be the least stage such that $\Gamma^{C \oplus W^{C}}(e)\left[s_{1}\right] \downarrow$. Then after stage $s_{1}$, $\gamma^{C \oplus W^{C}}(e)[s]$ will not move up, so $\lim _{s} \gamma^{C \oplus W^{C}}(e)[s]=\gamma^{C \oplus W^{C}}(e)\left[s_{1}\right]$.

\subsubsection{A $\mathcal{Q}_{e^{- \text {strategy }}}$}

Fix the canonical notation for $\omega^{2}$. That is, for each $\beta=\omega \cdot i+j$, we view $\beta$ coded as $\langle i, j\rangle$, and the corresponding order on the set of all ordered pairs of natural numbers is just the lexicographic order, i.e., $\langle i, j\rangle<\langle k, l\rangle$ iff $i<k$ or $i=k$ with $j<l$. Anderson and Csima proved in [1] that $\varnothing^{\dagger \dagger}$ is $\omega^{2}$-c.e. under this notation, i.e., there is a partial computable function $\chi: \omega \times \omega^{2} \rightarrow\{0,1\}$ such that

- for each $e$, there is a least ordinal $\beta_{e}<\omega^{2}$ such that $\chi\left(e, \beta_{e}\right) \downarrow=\varnothing^{\dagger \dagger}(e)$.

Then

- for each $e$, there is a first stage $s_{e}$ and a least ordinal $\beta_{e, s_{e}}=\omega \cdot i_{s_{e}}+j_{s_{e}}$ such that $\chi\left(e, \beta_{e, s_{e}}\right)\left[s_{e}\right] \downarrow$;

- for each $e$ and stage $s \geq s_{e}$, there is a least ordinal $\beta_{e, s}=\omega \cdot i_{e, s}+j_{e, s}$ such that $\chi\left(e, \beta_{e, s}\right)[s] \downarrow$.

Now $\beta_{e}=\lim _{s} \beta_{e, s}$, and $\varnothing^{\dagger \dagger}(e)=\chi\left(e, \beta_{e}\right)=\lim _{s} \chi\left(e, \beta_{e, s}\right)$. As $\chi$ is partial computable, $i_{s_{e}}$ and $j_{s_{e}}$ are computable functions on $e ; i_{e, s}$ and $j_{e, s}$ are computable functions on $e, s$.

For convenience, we can also choose $\chi$ such that

- for each $e$ and stage $s \geq s_{e}$, if $\beta_{e, s} \neq \beta_{e, s+1}$, then $\chi\left(e, \beta_{e, s+1}\right) \neq \chi\left(e, \beta_{e, s}\right)$.

In this case, $\beta_{e, s+1}<\beta_{e, s}$, that is, either $i_{e, s+1}<i_{e, s}$, or $i_{e, s+1}=i_{e, s}$ with $j_{e, s+1}<j_{e, s}$. 
Fix a partial computable function $\chi: \omega \times \omega^{2} \rightarrow\{0,1\}$ which satisfies conditions above. At stage $s \geq s_{e}$, we view $\varnothing^{\dagger \dagger}[s](e)=\chi\left(e, \beta_{e, s}\right)$.

Consider the requirement $\mathcal{Q}_{e}: \varnothing^{\dagger \dagger}(e)=\Delta^{C^{\dagger}} g_{g(e)}(e)$. At stage $s^{\prime} \geq s_{e}$, we define $\Delta^{C^{\dagger} g_{(e)}}(e)\left[s^{\prime}\right]=\chi\left(e, \beta_{e, s^{\prime}}\right)$. If there is a least stage $s>s^{\prime}$ at which $\beta_{e, s}<\beta_{e, s-1}$ or $\mathcal{Q}_{e}$ is injured by $\mathcal{R}$-strategies with higher priority (i.e., the current coding indicator of $\mathcal{Q}_{e}$ is less than or equal to the $C$-restraint on $\mathcal{R}$ ), then we need to rectify functional

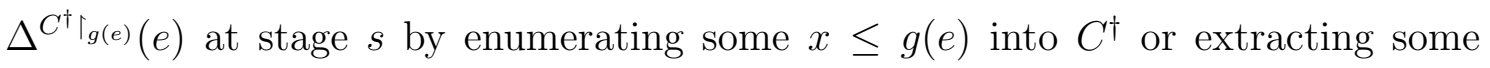
$y \leq g(e)$ out of $C^{\dagger}$.

In order to enumerate elements into or extract elements out of $C^{\dagger}$, we will build additional partial computable functions and partial computable functionals. By using the recursion theorem, we can change $C^{\dagger}$ on indices of such partial computable functionals. The construction indeed relies on parameters, say $r$. In the $r$-th construction, we will build an auxiliary partial computable function $q_{r}$, an auxiliary partial computable functional $\Theta_{r}$, and also an auxiliary c.e. set $Q_{r}$, where $Q_{r}$ encodes the graph of $q_{r}$ as well as the c.e. relation of $\Theta_{r}$.

Assume that we are in the $r$-th construction. To encode $\varnothing^{\dagger \dagger}(e)$ into $\Delta^{C^{\dagger}}(e)$, we will reserve three types of markers of $\mathcal{Q}_{e}$, namely injury markers, location markers and coding markers [such markers will be defined in Case 1 and Case 2 below]. We build the functional $\Delta$ such that $\Delta^{C^{\dagger}}(e)$ only depends on the membership of such markers in $C^{\dagger}$. Let $U_{e, r}$ be the set of all injury markers, location markers and coding markers defined during the $r$-th construction. Then the use function $\delta^{C^{\dagger}}(e)=\max U_{e, r}$. We will determine a strictly increasing computable function $g_{r}$ (in section 3.1.4) such that $g_{r}(e) \geq \max U_{e, r}$. Then $\varnothing^{\dagger \dagger}(e)=\Delta^{\left.C^{\dagger}\right|_{g_{r}(e)}}(e)$.

Case 1. We first consider the situation when $\mathcal{Q}_{e}$ is injured from negative requirements with higher priority.

Injury markers. When the $C$-restraint $r(i, s)$ on $\mathcal{R}_{i}(i \leq e)$ increases to a new level at stage $s$ with $r(i, s) \geq c$, the coding indicator of $\mathcal{Q}_{e}$ [the definition of coding indicators will be given in the following Case 2.1], we will enumerate an injury marker $<g_{r}(e)$ into $C^{\dagger}$ and never remove it out. This enumeration injures all old convergence $\Delta^{\left.C^{\dagger}\right|_{r}(e)}(e)$-computations, and $\Delta^{C^{\dagger} \eta_{g_{r}(e)}}(e)$ will arrive at new computations. In this case, just cancel existed coding indicators and coding markers of $\mathcal{Q}_{j}$ with $j \geq e$. 
In order to define injury markers, we first choose a fresh witness, $x$ say. Now $q_{r}(x)$ and $\Theta_{r}^{Y}(x)$ (for any oracle $Y$ ) are undefined currently. Define $q_{r}(x)=0$ and $\Theta_{r}^{Y}(x)=0$ for all oracles $Y$ at stage $s$, and then enumerate $\langle\langle x, 0\rangle,\langle\lambda, x, 0\rangle\rangle$ into $Q_{r, s}$ (note we also enumerate other elements of the form $\langle\langle y, c\rangle,\langle\sigma, y, 0\rangle\rangle$ with $\sigma$ a binary string into $Q_{r}$ when defining location markers and coding markers), where $\langle\cdot, \cdot\rangle: \mathbb{N}^{2} \rightarrow \mathbb{N}$ and $\langle\cdot, \cdot, \cdot\rangle: \mathbb{N}^{3} \rightarrow \mathbb{N}$ are fixed effective one-to-one functions; $\lambda$ stands for the empty string [we indeed fix an effective coding of $2^{<\omega}$ (the set of binary strings) into $\mathbb{N}$ and view a binary string $\sigma$ in $\langle\sigma, y, 0\rangle$ as its code].

We now provide more details for the use of recursion theorem.

The application of recursion theorem.

First, by $s_{n}^{m}$-theorem, there are computable functions $f_{1}$ and $f_{2}$ such that for each $x, W_{f_{1}(x)}=\left\{z: \exists y\left[\langle z, y\rangle \in W_{x}\right]\right\}$, and $W_{f_{2}(x)}=\left\{y: \exists z\left[\langle z, y\rangle \in W_{x}\right]\right\}$, where $W_{x}$ is the $x$-th c.e. set of natural numbers.

In the $r$-th construction, if $Q_{r}=W_{r}$, then

$$
W_{f_{1}(r)}=\left\{z: \exists y\left[\langle z, y\rangle \in Q_{r}\right]\right\}
$$

and

$$
W_{f_{2}(r)}=\left\{y: \exists z\left[\langle z, y\rangle \in Q_{r}\right]\right\} .
$$

So the graph of $q_{r}$ is $W_{f_{1}(r)}$, and the c.e. relation of $\Theta_{r}$ is just $W_{f_{2}(r)}$. Then again, we can use $s_{n}^{m}$-theorem as well as the relativized $s_{n}^{m}$-theorem to obtain the injective computable functions $k_{r}$ and $h_{r}$ such that for $n, m$ and $Y$,

$$
\varphi_{k_{r}(n)}(m)=q_{r}(n), \quad \Phi_{h_{r}(n)}^{Y}(m) \simeq \Theta_{r}^{Y}(n) .
$$

Moreover, by Padding Lemma, we can further choose $k_{r}$ and $h_{r}$ such that $k_{r}(n)<$ $h_{r}(n)$ for all $n$. Such $k_{r}$ and $h_{r}$ will be used to define injury markers, location markers as well as coding markers.

However, the assumption that $Q_{r}=W_{r}$ may be incorrect. Then our guess on $q_{r}$ and $\Theta_{r}$ are wrong, and hence $k_{r}$ and $h_{r}$ are also incorrect. Thus, the $r$-th construction does not work in general.

Observe that the c.e. sets $Q_{r}(r \in \mathbb{N})$ are uniform in $r$, then $Q_{r}=W_{f(r)}$ for some computable function $f$. By recursion theorem, there is at least one $r_{0}$ (indeed 
infinitely many) such that $Q_{r_{0}}=W_{f\left(r_{0}\right)}=W_{r_{0}}$. Then in the $r_{0}$-th construction, our guess on $q_{r_{0}}$ and $\Theta_{r_{0}}$ are both correct, and thus, the injective functions $k_{r_{0}}$ and $h_{r_{0}}$ really satisfy for all $n, m$ and $Y$,

$$
\varphi_{k_{r_{0}}(n)}(m)=q_{r_{0}}(n), \quad \Phi_{h_{r_{0}}(n)}^{Y}(m) \simeq \Theta_{r_{0}}^{Y}(n)
$$

Then $\mathcal{Q}_{e}$ can be satisfied via the $r_{0}$-th construction.

This ends the description of applying recursion theorem.

Now return back to the fresh witness $x$ above which devotes to define injury markers. At stage $s$, after defining $q_{r}(x)=0$ and $\Theta_{r}^{Y}(x)=0$ for all oracles $Y$, we view $\Phi_{h_{r}(x)}^{\varnothing}\left(h_{r}(x)\right) \downarrow=\Theta_{r}^{\varnothing}\left(h_{r}(x)\right) \downarrow=0$, and for all $m, \varphi_{k_{r}(x)}(m) \downarrow=q_{r}(x) \downarrow=0$. Then

$$
\Phi_{h_{r}(x)}^{C \Gamma_{\varphi_{k_{r}(x)}}\left(h_{r}(x)\right)}\left(h_{r}(x)\right) \downarrow \wedge k_{r}(x)<h_{r}(x) .
$$

This means that $h_{r}(x)$ enumerates into $C^{\dagger}$ forever. Call $h_{r}(x)$ an injury marker of $\mathcal{Q}_{e}$.

As we will determine an increasing computable function $g_{r}$ with $g_{r}(e)$ bigger than all injury markers of $\mathcal{Q}_{e}, \Delta^{C^{\dagger} g_{r(j)}}(j) \uparrow$ for all $j \geq e$. We can omit such $\mathcal{Q}_{j}$ 's coding indicators and coding markers, and $\mathcal{Q}_{j}$ will obtain new coding indicators larger than the $C$-restraint $r(i, s)$.

Case 2. We now consider the situation when $\beta_{e, s}<\beta_{e, s-1}$. There are two subcases.

Case 2.1. If the approximation of $\varnothing^{\dagger \dagger}(e)$ changes at stage $s$ via $i_{e, s}<i_{e, s-1}$, then we will undefine all old definitions and then define $\Delta^{C^{\dagger} \rho_{g_{r}}(e)}(e)$ via new computations.

Location markers. To undefine old computations, we will enumerate a new number $<g_{r}(e)$ into $C^{\dagger}$ and never remove it out. Such a number is called a location marker at level $i_{e, s}$, which indicates that approximations of $\varnothing^{\dagger \dagger}(e)$ move to new level $i_{e, s}$ and that $\Delta^{C^{\dagger} \rho_{r}(e)}(e)$ will be defined at that new level.

To define location markers, we choose a big number, say $y$, and then define $q_{r}(y)=0, \Theta_{r}^{Y}(y)=0$ for all oracles $Y$, and enumerate $\langle\langle y, 0\rangle,\langle\lambda, y, 0\rangle\rangle$ into $Q_{r, s}$. Then we view

$$
\Phi_{h_{r}(y)}^{C \varphi_{\varphi_{k_{r}(y)}\left(h_{r}(y)\right)}}\left(h_{r}(y)\right) \downarrow \wedge k_{r}(y)<h_{r}(y) ;
$$


by $\varphi_{k_{r}(y)}\left(h_{r}(y)\right)=0, h_{r}(y) \in C^{\dagger}[t]$ for all $t \geq s$. Say that $h_{r}(y)$ is a location marker at level $i_{e, s}$. Now $\Delta^{C^{\dagger} g_{r}(e)}(e)$ is undefined, because we will define $g_{r}(e)$ bigger than all location markers of $\mathcal{Q}_{e}$.

Coding markers and coding indicators. After obtaining location markers above, we immediately define coding markers by picking a new number, say $c$, and then define a coding marker via $c$ as follows. Again, choose a number $z$ larger than all used numbers. Define

$$
q_{r}(z)=c
$$

and

$$
\Theta_{r}^{Y}(z):= \begin{cases}0, & \text { if } c \notin Y \\ \uparrow, & \text { otherwise. }\end{cases}
$$

We enumerate all elements of the form $\langle\langle z, c\rangle,\langle\sigma, z, 0\rangle\rangle$ with $\sigma \in 2^{<\omega},|\sigma|=c+1$ and $\sigma(c)=0$ into $Q_{r, s}$, where $|\sigma|$ is the length of $\sigma$.

We think for all $m$,

$$
\varphi_{k_{r}(z)}(m) \downarrow=q_{r}(z) \downarrow=c, \quad \Phi_{h_{r}(z)}^{Y}(m) \simeq \Theta_{r}^{Y}(z) .
$$

So for all stages $t \geq s$,

$$
\Phi_{h_{r}(z)}^{C \Gamma_{c}}\left(h_{r}(z)\right)[t] \downarrow \Leftrightarrow \Theta_{r}^{C \Gamma_{c}}(z)[t] \downarrow \Leftrightarrow c \notin C_{t} .
$$

Then we view $h_{r}(z) \in C^{\dagger}[t] \Leftrightarrow c \notin C_{t}$. Such a $h_{r}(z)$ is called a coding marker at level $i_{e, s}$, and such a witness $c$ is called the coding indicator of $h_{r}(z)$.

We will define $\Delta^{C^{\dagger} g_{r}(e)}(e)$ via the coding marker $h_{r}(z)$ and the coding indicator $c$ at level $i_{e, s}$. If $\chi\left(e, \beta_{e, s}\right)=1$, then keep $c \notin C_{s}$ and define

$$
\Delta^{\left.C^{\dagger}\right|_{r}(e)}(e)[s]=C^{\dagger}[s]\left(h_{r}(z)\right)=1 .
$$

Otherwise, $\chi\left(e, \beta_{e, s}\right)=0$, to undefine $\Theta_{r}^{C_{s}}(z)$, just enumerate the coding indicator $c$ into $C_{s}$. Then $\Phi_{h_{r}(z)}^{C_{s}}(m) \uparrow$ for all $m$; in particular, $\Phi_{h_{r}(z)}^{C_{s}}\left(h_{r}(z)\right) \uparrow$, and thus $h_{r}(z) \notin C^{\dagger}[s]$. Define

$$
\Delta^{C^{\dagger} \wp_{r}(e)}(e)[s]=C^{\dagger}[s]\left(h_{r}(z)\right)=0 .
$$


Case 2.2. If $i_{e, s}=i_{e, s-1}$, but the approximation $\chi\left(e, \beta_{e, s}\right)$ of $\varnothing^{\dagger \dagger}(e)$ changes at stage $s$ via $j_{e, s}<j_{e, s-1}$. As the approximation stays in the previous level, we have defined the coding indicator $c$ and the coding marker $h_{r}(z)$ at level $i_{e, s}$, and defined

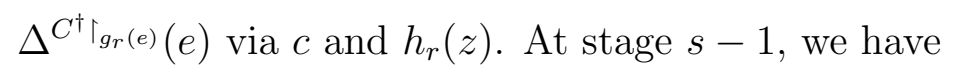

$$
\Delta^{\left.C^{\dagger}\right|_{r}(e)}(e)[s-1]=\chi\left(e, \beta_{e, s-1}\right)=C^{\dagger}[s-1]\left(h_{r}(z)\right)=1-C_{s-1}(c)
$$

If $\chi\left(e, \beta_{e, s}\right)=0$, then $\chi\left(e, \beta_{e, s-1}\right)=1$, so $c \notin C_{s-1}$. Just enumerate $c$ into $C_{s}$. If our construction is the $r_{0}$-th construction provided by recursion theorem, this enumeration undefines $\Phi_{h_{r_{0}}(z)}^{C_{s}}\left(h_{r_{0}}(z)\right)$, so $h_{r_{0}}(z) \notin C^{\dagger}[s]$. We can define

$$
\Delta^{C^{\dagger} \rho_{r_{0}}(e)}(e)[s]=0=\chi\left(e, \beta_{e, s}\right)=C^{\dagger}[s]\left(h_{r_{0}}(z)\right)=1-C_{s}(c) .
$$

If $\chi\left(e, \beta_{e, s}\right)=1$, then $\chi\left(e, \beta_{e, s-1}\right)=0$, so $c \in C_{s-1}$. Now extract $c$ out of $C$. Similarly, if we are in the $r_{0}$-th construction, then $\Phi_{h_{r_{0}}(z)}^{C \Gamma_{c}}\left(h_{r_{0}}(z)\right)[s] \downarrow$ with $\varphi_{k_{r_{0}}(z)}\left(h_{r_{0}}(z)\right)=c$ and $k_{r_{0}}(z)<h_{r_{0}}(z)$, and hence $h_{r_{0}}(z) \in C^{\dagger}[s]$. We can define

$$
\Delta^{C^{\dagger} g_{r_{0}}(e)}(e)[s]=1=\chi\left(e, \beta_{e, s}\right)=C^{\dagger}[s]\left(h_{r_{0}}(z)\right)=1-C_{s}(c) .
$$

\subsubsection{Define the computable function $g_{r}$}

We now specify a computable function $g_{r}$ in the $r$-th construction such that for all $e, g_{r}(e)$ is bigger than $\max U_{e, r}$, where $U_{e, r}$ is the set of injury markers, location markers and coding markers of $\mathcal{Q}_{e}$ defined in the $r$-th construction.

We first determine a recursive upper bound for the size $\left|U_{e, r}\right|$ of $U_{e, r}$. For injury markers, we define them only if some $\mathcal{R}_{i}(i \leq e)$ has its $C$-restraint $r(i, s)$ increase. So we also determine a number $\mu(i)$ which exceeds the number of times $r(i, s)$ increases.

As $\mathcal{R}_{0}$ has the highest priority, when 0 is enumerated into $W^{C}[s]$ for first time, we set $C$-restraint $r(0, s)=\psi^{C}(0)[s]$ and preserve it forever. Thus, $r(0, s)$ increases at most once, and let $\mu(0)=1$.

Now consider $\left|U_{0, r}\right|$. $\quad \mathcal{Q}_{0}$ is injured at most once when $r(0, s)$ increases, so it has at most one injury marker. For location markers, we define them when the approximation of $\varnothing^{\dagger \dagger}(0)$ decreases to a new level. Recall that $s_{0}$ is the first stage at which there is a least ordinal $\beta_{0, s_{0}}=\omega \cdot i_{s_{0}}+j_{s_{0}}$ such that $\chi\left(0, \beta_{0, s_{0}}\right)\left[s_{0}\right] \downarrow$, so there are at most $i_{s_{0}}+1$ many levels for $\mathcal{Q}_{0}$. Thus, the number of location markers of 
$\mathcal{Q}_{0}$ is at most $i_{s_{0}}$. For coding markers, we change them when $\mathcal{Q}_{0}$ is injured or the approximation of $\varnothing^{\dagger \dagger}(0)$ arrives at a new level, then the number of coding markers of $\mathcal{Q}_{0}$ (= the number of coding indicators of $\mathcal{Q}_{0}$ ) is at most $\mu(0)+i_{s_{0}}+1$. Hence,

$$
\left|U_{0, r}\right| \leq \mu(0)+i_{s_{0}}+\left(\mu(0)+i_{s_{0}}+1\right)
$$

In order to find an upper bound for general $\left|U_{e, r}\right|$, we also determine a number $\nu(e)$ which bounds the number of coding markers of $\mathcal{Q}_{e}$. Set $\nu(0)=\mu(0)+i_{s_{0}}+1$. Assume that we have already obtained $\mu\left(e^{\prime}\right)$ and $\nu\left(e^{\prime}\right)$ for all $e^{\prime}<e$.

Now determine $\mu(e)$ which bounds the number of times $C$-restraints $r(e, s)$ on $\mathcal{R}_{e}$ increases. $\mathcal{R}_{e}$ is injured at stage $s$ if some $\mathcal{P}_{i}(i<e)$ enumerates $\Gamma$-uses $\leq r(e, s)$ into $C$, or some $\mathcal{Q}_{i}(i<e)$ enumerates coding indicators $\leq r(e, s)$ into $C$ or removes coding indicators $\leq r(e, s)$ out of $C$. Note that each $\mathcal{P}_{i}(i<e)$ will enumerate at most one use into $C$. $\mathcal{R}_{e}$ will be injured by $\mathcal{P}$-requirements at most $2^{e}$ many times. For a sequence of fixed coding indicators, say $c_{i}$, of $\mathcal{Q}_{i}$ with $i<e$, the number of configurations of $C$ at this sequence is $2^{e}$, and there are at most $\nu(i)$ many coding indicators of $\mathcal{Q}_{i}(i<e)$. $\mathcal{R}_{e}$ will be injured by $\mathcal{Q}$-requirements for at most $2^{e} \prod_{0 \leq i<e} \nu(i)$ many times.

In total, $\mathcal{R}_{e}$ will be injured by positive requirements at most $2^{2 e} \prod_{0 \leq i<e} \nu(i)$ many times. Moreover, $r(e, s)$ can move up before or after each injury, hence, $r(e, s)$ will increase at most $2^{2 e+1} \prod_{0 \leq i<e} \nu(i)$ times. Just set $\mu(e)=2^{2 e+1} \prod_{0 \leq i<e} \nu(i)$.

Now we can define $\nu(e)$ which bounds the number of coding markers of $\mathcal{Q}_{e}$. We define coding markers for $\mathcal{Q}_{e}$ when it is injured by $\mathcal{R}_{i}(i \leq e)$ in which case $r(i, s)$ increases, or the approximation of $\varnothing^{\dagger \dagger}(e)$ arrives at a new level. Recall that $s_{e}$ is the first stage at which there is a least ordinal $\beta_{e, s_{e}}=\omega \cdot i_{s_{e}}+j_{s_{e}}$ such that $\chi\left(e, \beta_{e, s_{e}}\right)\left[s_{e}\right] \downarrow$. $\mathcal{Q}_{e}$ has at most $i_{s_{e}}+1$ many levels. Then we can set $\nu(e)=i_{s_{e}}+1+\sum_{0 \leq i \leq e} \mu(i)$.

Now we are ready to determine a recursive upper bound for $\left|U_{e, r}\right|$. $\mathcal{Q}_{e}$ has at most $i_{s_{e}}$ many location markers; and $\mathcal{Q}_{e}$ is injured by $\mathcal{R}_{i}$ with $i \leq e$ at most $\sum_{0 \leq i \leq e} \mu(i)$ many times, so it has at most $\sum_{0 \leq i \leq e} \mu(i)$ many injury markers; and $\mathcal{Q}_{e}$ has at most $\nu(e)$ many coding markers. Thus in total,

$$
\left|U_{e, r}\right| \leq i_{s_{e}}+\sum_{0 \leq i \leq e} \mu(i)+\nu(e) \leq 2 \nu(e)
$$


This ends the description of bounding the size of $U_{e, r}$.

Our initial target is to bound $\max U_{e, r}$, the use of $\Delta^{C^{\dagger}}(e)$. In order to recursively bound $\max U_{e, r}$, during the $r$-th construction, we will choose $\Gamma$-uses for $\mathcal{P}$ requirements and the witnesses at which we define $q_{r}$ and $\Theta_{r}$ for $\mathcal{Q}$-requirements in a neat way. Fix an effective injection $\langle\rangle:, \mathbb{N} \times \mathbb{N} \rightarrow \mathbb{N}$ such that for all $i, j \in \mathbb{N}$, $\langle i, j\rangle$ is strictly bigger than both $i$ and $j$, and if $i<k$ and $j<l$, then $\langle i, j\rangle$ is strictly less than $\langle k, l\rangle$. Let $\langle i, \mathbb{N}\rangle:=\{\langle i, j\rangle: j \in \mathbb{N}\}$.

All $\Gamma(e)$-uses for $\mathcal{P}_{e}$ are chosen from $\langle 3 e, \mathbb{N}\rangle . \Gamma(e)$-uses will be moved up only if some $i \leq e$ enumerates into $\varnothing^{\prime}$, or the $C$-restraints on some $\mathcal{R}_{i}$ with $i \leq e$ increases.

The witnesses for defining injury markers, location markers, and coding markers of $\mathcal{Q}_{e}$ are chosen from $\langle 3 e+1, \mathbb{N}\rangle$, and there are at most $2 \nu(e)$ many such witnesses. Then during the construction, we choose such witnesses of $\mathcal{Q}_{0}$ from

$$
\{\langle 1, x\rangle: x \leq 2 \nu(0)\}
$$

one by one in increasing order, and choose such witnesses of $\mathcal{Q}_{e}$ from

$$
\left\{\langle 3 e+1, x\rangle: e+2 \sum_{0 \leq i \leq e-1} \nu(i) \leq x \leq e+2 \sum_{0 \leq i \leq e} \nu(i)\right\}
$$

one by one in increasing order.

The coding indicators for coding markers of $\mathcal{Q}_{e}$ are chosen from $\langle 3 e+2, \mathbb{N}\rangle$, and there are at most $\nu(e)$ many such coding indicators. During the construction, when the $C$-restraint on some $\mathcal{R}_{i}(i \leq e)$ exceeds the coding indicators of $\mathcal{Q}_{e}$ or the approximations of $\varnothing^{\dagger \dagger}(e)$ decrease into a new level, we omit old coding indicators of $\mathcal{Q}_{e}$, and appoint new coding indicators form $\langle 3 e+2, \mathbb{N}\rangle$.

During the $r$-th construction:

(1) When defining injury markers or location markers for $\mathcal{Q}_{e}$, we first pick a least unused witness, say $\langle 3 e+1, y\rangle$, with $y$ satisfying:

$$
\begin{aligned}
& -e+2 \sum_{0 \leq i \leq e-1} \nu(i) \leq y \leq e+2 \sum_{0 \leq i \leq e} \nu(i), \\
& -q_{r}(\langle 3 e+1, y\rangle) \uparrow,
\end{aligned}
$$




$$
-\Theta_{r}^{Y}(\langle 3 e+1, y\rangle) \uparrow
$$

By $\left|U_{e, r}\right| \leq 2 \nu(e)$, during the $r$-th construction, we will define $q_{r}$ and $\Theta_{r}^{Y}$ on no more than $2 \nu(e)$ many arguments inside $\langle 3 e+1, \mathbb{N}\rangle$, so such a $y$ exists. We then define

$$
q_{r}(\langle 3 e+1, y\rangle)=0
$$

and

$$
\Theta_{r}^{Y}(\langle 3 e+1, y\rangle)=0
$$

for all oracles $Y$, and view

$$
\Phi_{h_{r}(\langle 3 e+1, y\rangle)}^{\varnothing}\left(h_{r}(\langle 3 e+1, y\rangle)\right) \downarrow=0 \wedge \varphi_{q_{r}(\langle 3 e+1, y\rangle) \downarrow=0}
$$

with $q_{r}(\langle 3 e+1, y\rangle)<h_{r}(\langle 3 e+1, y\rangle)$. That is, the injury marker or the location marker $h_{r}(\langle 3 e+1, y\rangle)$ is enumerated into $C^{\dagger}$ forever.

(2) When defining coding markers of $\mathcal{Q}_{e}$, we first choose a new coding indicator, say $\langle 3 e+2, x\rangle$, with $x$ bigger than all numbers used before, and then pick a least unused witness, say $\langle 3 e+1, z\rangle$, with $z$ satisfying:

$$
\begin{aligned}
& -e+2 \sum_{0 \leq i \leq e-1} \nu(i) \leq z \leq e+2 \sum_{0 \leq i \leq e} \nu(i), \\
& -q_{r}(\langle 3 e+1, z\rangle) \uparrow, \\
& -\Theta_{r}^{Y}(\langle 3 e+1, z\rangle) \uparrow .
\end{aligned}
$$

Similarly, such a $z$ exists. Then we define

$$
q_{r}(\langle 3 e+1, z\rangle)=\langle 3 e+2, x\rangle,
$$

and for all oracles $Y$, define

$$
\Theta_{r}^{Y}(\langle 3 e+1, z\rangle) \downarrow \Leftrightarrow\langle 3 e+2, x\rangle \notin Y .
$$

We view the coding marker $h_{r}(\langle 3 e+1, z\rangle)$ is enumerated into $C^{\dagger}$ iff its coding indicator $\langle 3 e+2, x\rangle$ is outside $C$. 
Hence, all markers of $\mathcal{Q}_{e}$ are of the form $h_{r}(\langle 3 e+1, y\rangle)$ for some

$$
e+2 \sum_{0 \leq i \leq e-1} \nu(i) \leq y \leq e+2 \sum_{0 \leq i \leq e} \nu(i)
$$

As $h_{r}$ is a computable function, we can define

$$
g_{r}(0)=\sum_{0 \leq y \leq 2 \nu(0)} h_{r}(\langle 1, y\rangle)
$$

For $e>0$, define $g_{r}(e)$ by induction as

$$
g_{r}(e)=1+g_{r}(e-1)+\sum_{e+2} \sum_{0 \leq i \leq e-1} \nu h_{r}(\langle 3 e+1, y\rangle)
$$

Then $g_{r}$ is a strictly increasing computable function such that for all $e, \max U_{e, r}<$ $g_{r}(e)$.

In the $r$-th construction, fix such a $g_{r}$ first, and for each $e$, we try to ensure $\varnothing^{\dagger \dagger}(e)=\Delta^{C^{\dagger} g_{r}(e)}(e)$ with the actual use $\delta^{C^{\dagger}}(e)=\max U_{e, r}$.

In the following formal construction and verification, assume that we are in the $r_{0}$-th construction, which is provided by recursion theorem. Then the injective computable functions $k_{r_{0}}, h_{r_{0}}, g_{r_{0}}$ are all correct, we denote them respectively as $k, h, g$ for simplicity.

\subsubsection{Construction}

Recall that $s_{e}$ is the first stage at which there is an ordinal $\alpha<\omega^{2}$ such that $\chi(e, \alpha)\left[s_{e}\right] \downarrow$. Assume $s_{e} \geq e$, and let $\beta_{e, s_{e}}=\omega \cdot i_{s_{e}}+j_{s_{e}}$ be the least such an $\alpha$. At stage $s \geq s_{e}$, let $\beta_{e, s}=\omega \cdot i_{e, s}+j_{e, s}$ be the least ordinal such that $\chi\left(e, \beta_{e, s}\right)[s] \downarrow$, and we assume $i_{e, s}, j_{e, s} \leq s$.

Assume that there is at most one element $\leq s$ enumerating into $\varnothing^{\prime}$ at each stage $s$.

\section{Construction of $C$.}

Let $C_{0}:=\varnothing$, and all functionals are totally undefined at stage 0 . 
Stage $s+1:$

(1) Coding $\varnothing^{\prime}$. If there is an $i \leq s+1$ such that $i \in \varnothing^{\prime}[s+1]-\varnothing^{\prime}[s]$ with $\Gamma^{C \oplus W^{C}}(i)[s] \downarrow$, then put $\gamma^{C \oplus W^{C}}(i)[s]$ into $C$. Thus, $\Gamma^{C \oplus W^{C}}(i)$ is undefined.

(2) Coding $\varnothing^{\dagger \dagger}$. Say $\mathcal{Q}_{e}$ requires attention at stage $s+1$ if $s+1 \geq s_{e}$ and one of the following conditions holds.

(Q0) $\mathcal{Q}_{e}$ has no coding indicators at the end of stage $s$.

(Q1) (Q0) fails and $\mathcal{Q}_{e}$ has an uncanceled coding indicator $c$, and some $\mathcal{R}_{i}$ with $i \leq e$ has its $C$-restraint $r(i, s) \geq c$.

(Q2) (Q0) and (Q1) both fail, and $\beta_{e, s+1}<\beta_{e, s}$ with $s+1>s_{e}$, in this case $\chi\left(e, \beta_{e, s+1}\right) \neq \chi\left(e, \beta_{e, s}\right)$.

Find all $\mathcal{Q}_{e}(e \leq s+1)$ which require attention at stage $s+1$, and consider them in order of priority.

Now assume that we arrive at $\mathcal{Q}_{e}$ which requires attention.

(2.1) When (Q0) holds.

- If there are no location markers at level $i_{e, s+1}$, first define a location marker at level $i_{e, s+1}$ as in $(\star)$ of (2.3.1).

- Define coding indicator and coding marker as in $(\star \star)$ of (2.3.1).

- Define computation $\Delta^{C^{\dagger} \Gamma_{g(e)}}(e)$ via the new coding indicator and coding marker as in $(\star \star \star)$ of $(2.3 .1)$.

Check whether there is a requirement $\mathcal{Q}_{j}(e<j \leq s+1)$ which requires attention at stage $s+1$. If there is such a $\mathcal{Q}_{j}$, find the highest priority one, act correspondingly. Otherwise, go to step (3). 
(2.2) When (Q1) holds.

First define an injury marker to undefine all old convergence computations of $\Delta^{C^{\dagger} g_{(e)}}(e)$ as follows:

- pick the least unused number $z$ in

$$
\left\{\langle 3 e+1, y\rangle: e+2 \sum_{0 \leq i \leq e-1} \nu(i) \leq y \leq e+2 \sum_{0 \leq i \leq e} \nu(i)\right\}
$$

- define $q_{r_{0}}(z)=0$ and $\Theta_{r_{0}}^{Y}(z)=0$ for all oracles $Y$.

By recursion theorem, for all $m, \varphi_{k(z)}(m) \downarrow=0, \Phi_{h(z)}^{\varnothing}(m) \downarrow=0$, we also choose $k(z)<h(z)$. So $h(z) \in C^{\dagger}[t]$ for all $t \geq s+1$. $h(z)$ is the desired injury marker.

As we choose $g$ such that $h(z)<g(e), \Delta^{\left.C^{\dagger}\right|_{g(e)}}(e) \uparrow$, thus all $\Delta^{\left.C^{\dagger}\right|_{g(j)}}(j) \uparrow$ with $j \geq e$. Say $\mathcal{Q}_{j}(j \geq e)$ is injured at stage $s+1$. Cancel all existed coding indicators and coding markers of $\mathcal{Q}_{j}(j \geq e)$, go to step (3).

(2.3) When (Q2) holds, $\beta_{e, s+1}<\beta_{e, s}$. There are two subcases (2.3.1) and (2.3.2).

(2.3.1) $i_{e, s+1}<i_{e, s}$, the approximation of $\varnothing^{\dagger \dagger}(e)$ now moves to a new level. Cancel the coding indicator and coding marker at level $i_{e, s}$. Define a location marker at level $i_{e, s+1}$ to undefine all old definitions of $\Delta^{C^{\dagger}} g_{g(e)}(e)$, and then define a coding marker to encode $\varnothing^{\dagger \dagger}[s+1](e)$, which is $\chi\left(e, \beta_{e, s+1}\right)$.

( ) Define the location marker. Pick the least unused number $z$ in

$$
\left\{\langle 3 e+1, y\rangle: e+2 \sum_{0 \leq i \leq e-1} \nu(i) \leq y \leq e+2 \sum_{0 \leq i \leq e} \nu(i)\right\}
$$

define $q_{r_{0}}(z)=0$ and $\Theta_{r_{0}}^{Y}(z)=0$ for all oracles $Y$. By recursion theorem, $h(z) \in C^{\dagger}[t]$ for all $t \geq s+1$, and $h(z)$ is the desired location marker. 
( $\star$ ) Define the coding indicator and the coding marker. First choose a coding indicator $c \in\langle 3 e+2, \mathbb{N}\rangle$ which is larger than all numbers used so far, and then pick the least unused number $x$ in

$$
\left\{\langle 3 e+1, y\rangle: e+2 \sum_{0 \leq i \leq e-1} \nu(i) \leq y \leq e+2 \sum_{0 \leq i \leq e} \nu(i)\right\} .
$$

Define $q_{r_{0}}(x)=c$ and $\Theta_{r_{0}}^{Y}(x) \downarrow \Leftrightarrow c \notin Y$ for all oracles $Y$. By recursion theorem, $h(x) \in C^{\dagger}[t]$ iff $c \notin C_{t}$ for all $t \geq s+1$. $h(x)$ is the desired coding marker with the coding indicator $c$.

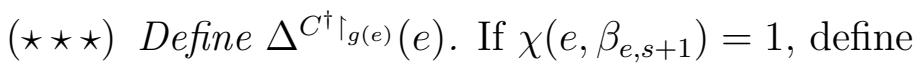

$$
\Delta^{\left.C^{\dagger}\right|_{(e)}}(e)[s+1]=C^{\dagger}[s+1](h(x))=1-C_{s+1}(c)=1 .
$$

Otherwise, $\chi\left(e, \beta_{e, s+1}\right)=0$. First enumerate the coding indicator $c$ into $C_{s+1}$, so $C^{\dagger}[s+1](h(x))=0$, and then define

$$
\Delta^{\left.C^{\dagger}\right|_{g(e)}}(e)[s+1]=C^{\dagger}[s+1](h(x))=1-C_{s+1}(c)=0 .
$$

(2.3.2) $i_{e, s+1}=i_{e, s}$ and $j_{e, s+1}<j_{e, s}$, the approximation of $\varnothing^{\dagger \dagger}(e)$ changes at same level. Let $c$ be the uncanceled coding indicator at level $i_{e, s}$, and $h(x)$ be the corresponding coding marker.

If $\chi\left(e, \beta_{e, s+1}\right)=1$, then $\chi\left(e, \beta_{e, s}\right)=0$. As

$$
\Delta^{C^{\dagger}{ }_{g(e)}}(e)[s]=C^{\dagger}[s](h(x))=1-C_{s}(c)=0,
$$

$c \in C_{s}$. Now remove $c$ out of $C$, then

$$
\Delta^{\left.C^{\dagger}\right|_{g(e)}}(e)[s+1]=C^{\dagger}[s+1](h(x))=1-C_{s+1}(c)=1 .
$$

If $\chi\left(e, \beta_{e, s+1}\right)=0$, then $\chi\left(e, \beta_{e, s}\right)=1$, and

$$
\Delta^{C^{\dagger} g_{g(e)}}(e)[s]=C^{\dagger}[s](h(x))=1-C_{s}(c)=1,
$$

So $c \notin C_{s}$. Now enumerate $c$ into $C$. Then

$$
\Delta^{\left.C^{\dagger}\right|_{g(e)}}(e)[s+1]=C^{\dagger}[s+1](h(x))=1-C_{s+1}(c)=0 .
$$


Check whether there is a requirement $\mathcal{Q}_{j}(e<j \leq s+1)$ which requires attention at stage $s+1$. If there is such a $\mathcal{Q}_{j}$, find the highest priority one, act correspondingly. Otherwise, go to step (3).

(3) Defining $\Gamma^{C \oplus W^{C}}$. Now we obtain $C_{s+1}$. Calculate $C$-restraints

$$
r(i, s+1)=\max \left\{\psi^{C}(i)[t]: t \leq s+1 \wedge \Psi^{C}(i)[t] \downarrow\right\}
$$

on each $\mathcal{R}_{i}$ with $i \leq s+1$.

- If there is a $r(i, s+1)$ such that $r(i, s+1)>r(i, s)$, then

$$
i \in W^{C}[s+1]-W^{C}[s]
$$

and $\Psi^{C}(i)[s+1]$ is defined with a new computation. If $\Gamma^{C \oplus W^{C}}(i)[s] \downarrow$,

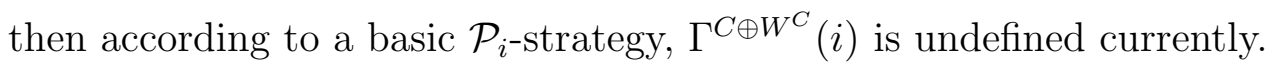

When we define $\Gamma^{C \oplus W^{C}}(i)$ at stage $t \geq s+1$, its use $\gamma^{C \oplus W^{C}}(i)[t]$ will be of the form $\langle 3 e, x\rangle$ with $x$ larger than all numbers used so far, especially, $\gamma^{C \oplus W^{C}}(i)[t]>r(i, s+1)$.

Find the least $y$ with $\Gamma^{C \oplus W^{C}}(y) \uparrow$ currently.

If $\Gamma^{C \oplus W^{C}}(y)$ is never defined before,

- define $\Gamma^{C \oplus W^{C}}(y)[s+1]=\varnothing^{\prime}[s+1](y)$ with use $\langle 3 e, x+1\rangle$, where $x$ is the least number larger than all numbers used so far.

Otherwise, let $w<s+1$ be the largest stage at which $\Gamma^{C \oplus W^{C}}(y)[w] \downarrow$.

- If $y \in \varnothing^{\prime}[w+1]-\varnothing^{\prime}[w]$, or

$\exists t \in[w, s]$ such that $y \in W^{C}[t+1]-W^{C}[t]$ with $r(y, t+1)>r(y, t)$, or $\gamma^{C \oplus W^{C}}(y)[w] \leq \gamma^{C \oplus W^{C}}(y-1)[s]$,

then define $\Gamma^{C \oplus W^{C}}(y)[s+1]=\varnothing^{\prime}[s+1](y)$ with use $\langle 3 e, x+1\rangle$, where $x$ is the least number larger than all numbers used so far. 
- Otherwise, $\gamma^{C \oplus W^{C}}(y)[w]>\gamma^{C \oplus W^{C}}(y-1)[s]$, just define $\Gamma^{C \oplus W^{C}}(y)[s+1]=$ $\varnothing^{\prime}[s+1](y)$ with use $\gamma^{C \oplus W^{C}}(y)[w]$.

This ends the construction of $C$ at stage $s+1$.

\subsubsection{Verification}

Here, we verify the $r_{0}$-th construction which is provided by the recursion theorem. Recall that we have already defined computable function $g(e)$, which is $g_{r_{0}}(e)$, in a basic $\mathcal{Q}_{e}$-strategy with $g(e) \geq \delta^{C^{\dagger}}(e)=\max U_{e, r_{0}}$, where $U_{e, r_{0}}$ is the set of injury markers, location markers and coding markers of $\mathcal{Q}_{e}$ defined in the $r_{0}$-th construction.

Lemma 3.1 For each requirement, $Q$ say,

(1) $Q$ is injured at most finitely often;

(2) $Q$ acts at most finitely often and is satisfied;

(3) Q injures all lower priority strategies finitely often.

Proof: We will prove the lemma by induction on $e$ that all $\mathcal{R}_{e}, \mathcal{P}_{e}$, and $\mathcal{Q}_{e}$ are true.

For $e=0$. $\mathcal{R}_{0}$ has the highest priority, it is not injured. (1) holds. If $0 \in$ $W^{C}[s]-W^{C}[s-1]$ at some first stage $s$, then $r(0, s)=\psi^{C}(0)[s]$ and the computation $\Psi^{C}(0)[s]$ is never destroyed later [indeed, after stage $s$, all $\mathcal{P}_{j}$ and $\mathcal{Q}_{j}$ with $j \geq 0$ can only deal with witnesses larger than $r(0, s)]$. Then $0 \in W^{C}[t]$ for all $t \geq s$. So $\mathcal{R}_{0}$ is satisfied, (2) holds. $\mathcal{R}_{0}$ acts at most once by setting $C$-restraints, thus initializes lower priority strategies at most once. (3) holds.

For $\mathcal{P}_{0} . \quad \mathcal{P}_{0}$ is injured by $\mathcal{R}_{0}$ at most once when $0 \in W^{C}[s]-W^{C}[s-1]$ at some first stage $s$, (1) holds. In this case, $\Gamma^{C \oplus W^{C}}(0) \uparrow$, we will define it with a big use later. Let $w_{0}$ be the least stage such that $\mathcal{P}_{0}$ is not injured after stage $w_{0}$. After stage $w_{0}, \mathcal{P}_{0}$ acts at most once if $0 \in \varnothing^{\prime}[t]-\varnothing^{\prime}[t-1]$ at some stage $t$ with $\Gamma^{C \oplus W^{C}}(0)[t-1] \downarrow$. In this case, $\gamma^{C \oplus W^{C}}(0)[t-1]$ is enumerated into $C_{t}$, and we will define $\Gamma^{C \oplus W^{C}}(0)=1$ with a large use later. So $\gamma^{C \oplus W^{C}}(0)$ increases at most twice 
after its first definition, there is a stage $w_{1} \geq w_{0}$ such that $\Gamma^{C \oplus W^{C}}(0)\left[w_{1}\right]$ is defined and is never undefined later. (2) holds. $\mathcal{P}_{0}$ never acts after stage $w_{1}$. (3) holds.

For $\mathcal{Q}_{0}$. Recall that we use $s_{0}$ to denote the first stage at which there is an ordinal $\alpha<\omega^{2}$ such that $\chi(0, \alpha)\left[s_{0}\right] \downarrow$, and let $\beta_{0, s_{0}}=\omega \cdot i_{s_{0}}+j_{s_{0}}$ be the least such ordinals. We will appoint a coding indicator $c$ at level $i_{s_{0}}$, and then define corresponding coding marker $h(z)$. Define

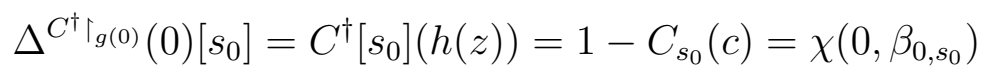

for first time.

When $0 \in W^{C}[s]-W^{C}[s-1]$ at some first stage $s \geq s_{0}$ with $r(0, s)$ bigger than coding indicators of $\mathcal{Q}_{0}, \mathcal{Q}_{0}$ is injured. We will enumerate an injury marker into

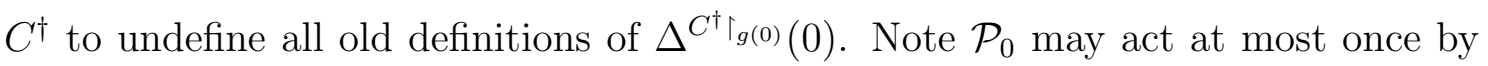
enumerating $\gamma^{C \oplus W^{C}}(0)$ into $C$, but this enumeration has no essential influences to the definition of $\Delta^{\left.C^{\dagger}\right|_{(0)}}(0)$ by the choice of $\Gamma$-uses and coding indicators. So $\mathcal{Q}_{0}$ is injured at most once by $\mathcal{R}_{0}$. (1) holds.

Let $t_{0} \geq s_{0}$ be the least stage such that $\mathcal{Q}_{0}$ is not initialized after stage $t_{0}$, and let $\beta_{0, t}=\omega \cdot i_{0, t}+j_{0, t}$ be the least ordinal such that $\chi\left(0, \beta_{0, t}\right)[t] \downarrow$ at stage $t \geq s_{0}$.

After stage $t_{0}$, if $\beta_{0, s}<\beta_{0, s-1}$ via $i_{0, s}<i_{0, s-1}$, then all the previous definitions of $\Delta^{C^{\dagger}{ }_{g(0)}(0)}$ are injured at stage $s$, because we enumerate a location marker at level $i_{0, s}$ into $C^{\dagger}$. Now assume that after stage $t_{1} \geq t_{0}, i_{0, t}$ never decreases, i.e., $i_{0, t}=i_{0, t_{1}}$ for all $t \geq t_{1}$ (such a $t_{1}$ exists since $\lim _{s} \beta_{0, s}$ exists).

Let $c^{\prime}$ be the coding indicator at level $i_{0, t_{1}}$ and $h\left(z^{\prime}\right)$ be the corresponding coding marker. We define $h\left(z^{\prime}\right)$ such that $h\left(z^{\prime}\right) \in C^{\dagger}[s] \Leftrightarrow c^{\prime} \notin C_{s}$ for all stage $s \geq t_{1}$. At stage $t_{1}$, we maintain

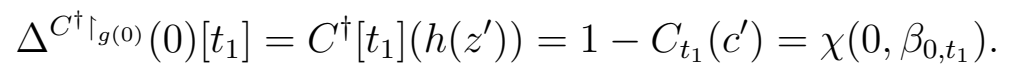

When $j_{0, t}<j_{0, t-1}$ at some stage $t>t_{1}$, we will enumerate $c^{\prime}$ into $C_{t}$ if $\chi\left(0, \beta_{0, t-1}\right)=$ 1 , and extract $c^{\prime}$ out of $C_{t-1}$ if $\chi\left(0, \beta_{0, t-1}\right)=0$, keeping

$$
\Delta^{C^{\dagger}{ }_{g(0)}(0)[t]}=C^{\dagger}[t]\left(h\left(z^{\prime}\right)\right)=1-C_{t}\left(c^{\prime}\right)=\chi\left(0, \beta_{0, t}\right) .
$$

Let $t_{2} \geq t_{1}$ be the least stage such that $j_{0, t}=j_{0, t_{2}}$ for all $t \geq t_{2}$ (again, such a $t_{2}$ exists since $\lim _{s} \beta_{0, s}=\beta_{0}$; in this case, $\left.\beta_{0}=\omega \cdot i_{0, t_{1}}+j_{0, t_{2}}\right)$. Then for all $t \geq t_{2}$, 
$\beta_{0, t}=\beta_{0, t_{2}}$ and $\varnothing^{\dagger \dagger}(0)=\chi\left(0, \beta_{0, t}\right)$. So $\mathcal{Q}_{0}$ never acts after stage $t_{2}$. Hence,

$$
\Delta^{C^{\dagger} I_{(0)}}(0)=C^{\dagger}\left[t_{2}\right]\left(h\left(z^{\prime}\right)\right)=1-C_{t_{2}}\left(c^{\prime}\right)=\chi\left(0, \beta_{0, t_{2}}\right)=\varnothing^{\dagger \dagger}(0) .
$$

We have showed that $\mathcal{Q}_{0}$ is satisfied after finitely many actions, and thus injures lower priority strategies finitely often. (2) and (3) hold.

Recall that $s_{l}$ is the first stage at which there is an ordinal $\alpha<\omega^{2}$ such that $\chi(l, \alpha)\left[s_{l}\right] \downarrow$. Let $\beta_{l, s}=\omega \cdot i_{l, s}+j_{l, s}$ be the least ordinal such that $\chi\left(l, \beta_{l, s}\right)[s] \downarrow$ at stage $s \geq s_{l}$.

Assume that the lemma is true for $\mathcal{R}_{i}, \mathcal{P}_{i}$ and $\mathcal{Q}_{i}$ with $i<e$. Let $v_{e}>\max \left\{s_{i}\right.$ : $i \leq e\}$ be the least stage such that for $i<e$,

- $\lim _{s} r(i, s)=r\left(i, v_{e}\right)$,

- $\Gamma^{C \oplus W^{C}}(i)\left[v_{e}\right]$ is defined and is never undefined after stage $v_{e}$,

- $\Delta^{C^{\dagger} g_{g(i)}}(i)\left[v_{e}\right]$ is defined with value $\chi\left(i, \beta_{i, v_{e}}\right)$ and is never changed later.

Then no $\mathcal{R}_{i}$ with $i<e$ has $C$-restraints $r(i, s)$ increase after stage $v_{e}, \lim _{s} \gamma^{C \oplus W^{C}}(i)[s]=$ $\gamma^{C \oplus W^{C}}(i)\left[v_{e}\right]$ for all $i<e$, and $\lim _{s} \beta_{i, s}=\beta_{i, v_{e}}$ for all $i<e$.

For $\mathcal{R}_{e}$. As no $\Gamma$-uses or coding indicators of positive requirements are enumerated into or removed out of $C$ after stage $v_{e}, \mathcal{R}_{e}$ is not injured after stage $v_{e}$ holds. After stage $v_{e}$, there are two cases.

(i) $r(e, s)$ never increases after stage $v_{e}$. Then $\lim _{s} r(e, s)=r\left(e, v_{e}\right), \mathcal{R}_{e}$ is satisfied and never acts after stage $v_{e}$.

(ii) There is a least stage $t>v_{e}$ at which $e \in W^{C}[t]-W^{C}[t-1]$ with new computation. Then $r(e, t)>r\left(e, s^{\prime}\right)$ for all $s^{\prime} \leq t-1$. During the construction, no positive requirements with lower priority will enumerate numbers $\leq r(e, t)$ into $C$ or extract numbers $\leq r(e, t)$ from $C$ after stage $t$, so $\Psi^{C}(0)[t]$ is never injured after stage $t$. Hence, $e \in W^{C}\left[s^{\prime}\right]$ for all $s^{\prime} \geq t$. $\mathcal{R}_{e}$ acts at most once after stage $v_{e}$ and is satisfied. 
Hence, (2) and (3) hold.

Similar to $\mathcal{P}_{0}$ and $\mathcal{Q}_{0}$ below $\mathcal{R}_{0}$. After stage $v_{e}$, for $\mathcal{P}_{e}$ and $\mathcal{Q}_{e}$ below $\mathcal{R}_{e}, \mathcal{P}_{e}$ is injured at most once, and acts at most once by enumerating $\gamma^{C \oplus W^{C}}(e)$ into $C$ when $e$ goes into $\varnothing^{\prime} ; \mathcal{Q}_{e}$ is injured at most once, and will be satisfied after finitely many actions. So the lemma holds for $\mathcal{P}_{e}$ and $\mathcal{Q}_{e}$.

Lemma 3.2 For all $x,\left|\left\{s: C_{s}(x) \neq C_{s+1}(x)\right\}\right| \leq x+1$. Thus $C$ is $\omega$-c.e.

Proof: We can effectively check whether $x$ is a $\Gamma$-use or a coding indicator or not. If no, $C_{s}(x)=0$ for all stages $s$.

Assume that $x$ is a $\Gamma(i)$-use for some $i$. It enumerates into $C$ at most once when $i$ goes into $\varnothing^{\prime}$, so $\left|\left\{s: C_{s}(x) \neq C_{s+1}(x)\right\}\right| \leq 1$.

Assume that $x$ is a coding indicator of some $\mathcal{Q}_{e}$ appointed at stage $s$. Consider the least ordinal $\beta_{e, s}=\omega \cdot i_{e, s}+j_{e, s}$ such that $\chi\left(e, \beta_{e, s}\right)[s] \downarrow$. Then $x>j_{e, s}$, and $x$ may be enumerated into $C_{s}$. At stage $t>s, C_{t}(x) \neq C_{t-1}(x)$ only if $j_{e, t}<j_{e, t-1} \leq j_{e, s}$. So $\left|\left\{s: C_{s}(x) \neq C_{s+1}(x)\right\}\right| \leq 1+j_{e, s} \leq x$.

By Lemma 3.1, $\varnothing^{\dagger \dagger} \leq_{w t t} C^{\dagger}, \varnothing^{\prime} \leq_{T} C \oplus W^{C}$, and $W^{C} \leq_{T} \varnothing^{\prime}$. By Lemma 3.2, $C$ is $\omega$-c.e. So $C$ is a bounded-high set with $C \oplus W^{C} \equiv_{T} \varnothing^{\prime}$.

This completes the proof of Theorem 3.1.(1).

\subsection{Pseudo-jump inversion via bounded-low c.e. sets}

To prove Theorem 3.1.(2), for the c.e. operator $W$, we need to build a bounded-low c.e. set $C$ (i.e., $C^{\dagger}$ is $\omega$-c.e.) such that $C \oplus W^{C} \equiv_{T} \varnothing^{\prime}$. As $C$ will be c.e., $C^{\dagger}$ has the following natural computable approximations:

$$
f_{C^{\dagger}}(x, s):= \begin{cases}1, & \text { if } \exists n \leq x\left[\varphi_{n}(x)[s] \downarrow \wedge \Phi_{x}^{C \mid \varphi_{n}(x)}(x)[s] \downarrow\right] ; \\ 0, & \text { otherwise. }\end{cases}
$$

We only need to add negative requirements of the form

$\mathcal{N}_{e}:\left|\left\{s: f_{C^{\dagger}}(e, s) \neq f_{C^{\dagger}}(e, s+1)\right\}\right| \leq 2(e+1)^{2}+1$ 
to the standard pseudo-jump inversion theorem. Then $C$ will be bounded-low.

We will build a functional $\Gamma$ such that $\Gamma^{C \oplus W^{C}}=\varnothing^{\prime}$. If $e$ enumerates into $\varnothing^{\prime}$, then rectify $\Gamma^{C \oplus W^{C}}(e)=1$ by enumerating $\gamma^{C \oplus W^{C}}(e)$ into $C$. To make $W^{C} \leq_{T} \varnothing^{\prime}$ (i.e., $W^{C}$ is $\left.\Delta_{2}^{0}\right)$, as usual, we will preserve $e \in W^{C}$ by setting a $C$-restraint $\psi^{C}(e)$ if $\Psi^{C}(e) \downarrow$.

When defining $\Gamma^{C \oplus W^{C}}$, the $\Gamma$-uses satisfy the following rules.

(i) For each $e$, and stage $s$, if $\Gamma^{C \oplus W^{C}}(e)$ is newly defined at stage $s$, its use $\gamma^{C \oplus W^{C}}(e)[s]$ is defined to be larger than all numbers used before, especially, $\gamma^{C \oplus W^{C}}(e)[s]>e$.

(ii) For each $e$, and stage $s$, if $\Gamma^{C \oplus W^{C}}(e)[s] \downarrow$, then $\Gamma^{C \oplus W^{C}}(e)[t]$ is undefined at some stage $t>s$ only if

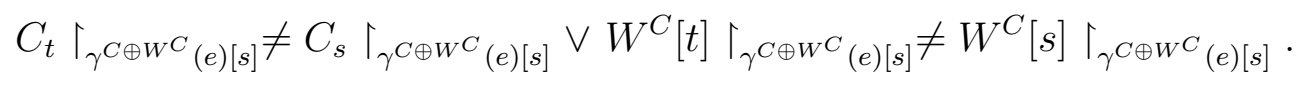

(iii) For $e<e^{\prime}$ and stage $s$, if $\Gamma^{C \oplus W^{C}}(e)[s]$ and $\Gamma^{C \oplus W^{C}}\left(e^{\prime}\right)[s]$ are both defined, then

$$
\gamma^{C \oplus W^{C}}(e)[s]<\gamma^{C \oplus W^{C}}\left(e^{\prime}\right)[s] .
$$

(iv) For each $e$, there is a stage $s_{e}$ such that for all $s \geq s_{e}, \Gamma^{C \oplus W^{C}}(e)[s] \downarrow=\varnothing^{\prime}[s](e)$ with use $\gamma^{C \oplus W^{C}}(e)[s]=\gamma^{C \oplus W^{C}}(e)\left[s_{e}\right]$.

This ensures that $\varnothing^{\prime}=\Gamma^{C \oplus W^{C}}$, and thus $\varnothing^{\prime} \leq_{T} C \oplus W^{C}$.

As in the standard pseudo-jump inversion theorem, the definition of $\Gamma^{C \oplus W^{C}}(e)$ is compatible with preserving $W^{C}(e)$.

Now consider the bounded-low requirement $\mathcal{N}_{e}$. In order to preserve $C^{\dagger}(e)$, at each stage $s$, we need to preserve a $C$-restraint

$$
r^{\mathcal{N}}(e, s)=\max \left\{\varphi_{n}(e)[s]: n \leq e \wedge \varphi_{n}(e)[s] \downarrow\right\}
$$

If $\Gamma^{C \oplus W^{C}}(e)[s] \downarrow$ with $\gamma^{C \oplus W^{C}}(e)[s] \leq r^{\mathcal{N}}(e, s), \mathcal{N}_{e}$ will act to enumerate $\gamma^{C \oplus W^{C}}(e)[s]$ into $C$, thus making $\Gamma^{C \oplus W^{C}}(e)$ undefined; in this case, $\Gamma^{C \oplus W^{C}}(e)$ will be redefined 
with a new use $>r^{\mathcal{N}}(e, s)$. Since $r^{\mathcal{N}}(e, s)$ can move up at most $e+1$ times, $\mathcal{N}_{e}$ will enumerate at most $e+1$ many such uses.

Now computations of the form $\Phi_{e}^{C \Gamma_{\varphi_{n}(e)}}(e)[s] \downarrow$ (of course, $\varphi_{n}(e)[s] \downarrow$ ) for some $n \leq$ $e$ are injured at stage $s+1$ only if there is some $i \leq e$ such that $\gamma^{C \oplus W^{C}}(i)[s] \leq \varphi_{n}(e)$ is enumerated into $C_{s+1}$. Now $\Gamma^{C \oplus W^{C}}(i) \uparrow$ currently, it will be defined with new use $>\varphi_{n}(e)$. So the enumeration of $\gamma^{C \oplus W^{C}}(i)$ later will not injure a new computation of the form $\Phi_{e}^{C \varphi_{\varphi_{n}(e)}}(e)$, and the enumeration of $\gamma^{C \oplus W^{C}}(i)$-uses can injure $\Phi_{e}^{C \varphi_{\varphi_{n}(e)}}(e)$ at most once for fixed $i$ and $n$. Thus, approximations for $C^{\dagger}(e)$ are injured at most $(e+1)^{2}$ many times. Note that each such an injury entails at most two changes of $f_{C^{\dagger}}(e, s)$. So

$$
\left|\left\{s: f_{C^{\dagger}}(e, s) \neq f_{C^{\dagger}}(e, s+1)\right\}\right| \leq 2(e+1)^{2}+1 \text {. }
$$

Hence, $C^{\dagger}$ is $\omega$-c.e., i.e., $C$ is bounded-low.

This completes the description of basic strategies and possible interactions between them. We omit the remaining construction and verification, which are finite injury arguments.

This completes the proof of Theorem 3.1.(2). 


\section{Part II}

\section{Degrees of orders on torsion-free abelian groups}




\section{Chapter 4}

\section{Group-order-computable degrees and PA degrees}

In Proposition 1.5, we have proved that PA degrees and degrees which are not weakly group-order-computable coincide. Then all PA degrees are not group-ordercomputable. In this chapter, we will show that the converse is also true, that is, degrees which are not PA are group-order-computable.

Theorem 4.1 There is a computable torsion-free abelian group $\mathscr{G}$ with infinite rank such that for any set $A, \mathscr{G}$ admits exactly two A-computable orders iff $A$ is not PAcomplete.

For $\mathscr{G}$ above, as any computable set is not PA-complete, $\mathscr{G}$ admits exactly two computable orders. Then for a set $A$ which is not PA-complete, every $A$-computable order on $\mathscr{G}$ is computable; for a set $A$ which is PA-complete, $A$ computes a noncomputable order on $\mathscr{G}$.

Let $\mathbf{a}$ be a degree which is not group-order-computable. Then for the group $\mathscr{G}$ in Theorem 4.1, a computes a noncomputable order on $\mathscr{G}$. Thus, a is a PA degree.

Now we obtain the following characterizations of PA degrees.

Proposition 4.1 Let a be a Turing degree. The following are equivalent.

(1) a is a PA degree.

(2) a is not weakly group-order-computable.

(3) a is not group-order-computable. 
Note that $\mathbf{0}^{\prime}$ is the only c.e. degree which is PA. An immediate corollary of Theorem 4.1 is:

Corollary 4.1 There is a computable torsion-free abelian group $\mathscr{G}$ with infinite rank such that

(1) $\mathscr{G}$ has exactly two computable orders;

(2) for any c.e. degree $\mathbf{a}<\mathbf{0}^{\prime}$, every $\mathbf{a}$-computable order on $\mathscr{G}$ is computable.

The following sections are devoted to prove Theorem 4.1.

\subsection{Requirements}

To prove Theorem 4.1, we will build a computable group $\mathscr{G}$ and a computable order $\leq \mathscr{G}$ on $\mathscr{G}$ to meet the following requirements.

$\mathcal{P}: \mathscr{G} \cong \mathbb{Q}^{\infty}$

$\mathcal{Q}: \leq_{\mathscr{G}}$ and $\leq_{\mathscr{G}}^{*}$ are exactly two computable orders on $\mathscr{G}$.

$\mathcal{R}$ : For any set $A$,

(1) if $\leq^{A}$ is an $A$-computable order on $\mathscr{G}$, then either $\leq^{A}=\leq_{\mathscr{G}}$, or $\leq^{A}=\leq_{\mathscr{G}}^{*}$, or $A$ is PA-complete;

(2) if $A$ is PA-complete, then there is an $A$-computable order on $\mathscr{G}$ which is not computable.

Here, $\mathbb{Q}^{\infty}$ is the direct sum of infinitely many copies of $\mathbb{Q} . \leq_{\mathscr{G}}^{*}$ denotes the reversal order of $\leq_{\mathscr{G}}$, that is, for $x, y \in \mathscr{G}, x \leq_{\mathscr{G}}^{*} y \Leftrightarrow y \leq_{\mathscr{G}} x$.

If three requirements above are satisfied, then $\mathscr{G}$ is a computable group with exactly two computable orders. Moreover, for any set $A$, if $A$ is not PA-complete, then every $A$-computable order on $\mathscr{G}$ is computable, so there are exactly two $A$ computable orders on $\mathscr{G}$; if $A$ is PA-complete, then there are $A$-computable orders which are not computable, so there are more than two $A$-computable orders on $\mathscr{G}$. 


\subsection{Background}

We now review a few concepts on binary strings which are needed for constructing $\mathscr{G}$. Let $2^{<\omega}$ be the set of binary strings. For each binary string $\sigma$, the symbol $|\sigma|$ denotes the length of $\sigma$. For the empty string $\lambda,|\lambda|=0$. For a nonempty string $\sigma,|\sigma| \geq 1$; in this case, write $\sigma:=\sigma(0) \cdots \sigma(|\sigma|-1)$ and for $1 \leq i \leq|\sigma|$, let $\sigma \uparrow_{i}:=\sigma(0) \cdots \sigma(i-1)$. Set $\sigma \uparrow_{0}:=\lambda$.

$2^{<\omega}$ has a natural lexicographical order $\leq_{l e x}$ defined as follows: $0<_{l e x} 1$, and for $\sigma, \tau \in 2^{<\omega}, \sigma \leq_{l e x} \tau$ if either there is an $i<\min (|\sigma|,|\tau|)$ with $\sigma \uparrow_{i}=\tau \uparrow_{i}$ and $\sigma(i)<_{\text {lex }} \tau(i)$; or $\sigma \subseteq \tau$. Another well-known order, the length-lexicographical order $\leq_{l e n-l e x}$, on $2^{<\omega}$ is defined like this: for $\sigma, \tau \in 2^{<\omega}$,

$$
\sigma \leq_{\text {len-lex }} \tau \Leftrightarrow|\sigma|<|\tau| \vee\left(|\sigma|=|\tau| \wedge \sigma \leq_{\text {lex }} \tau\right)
$$

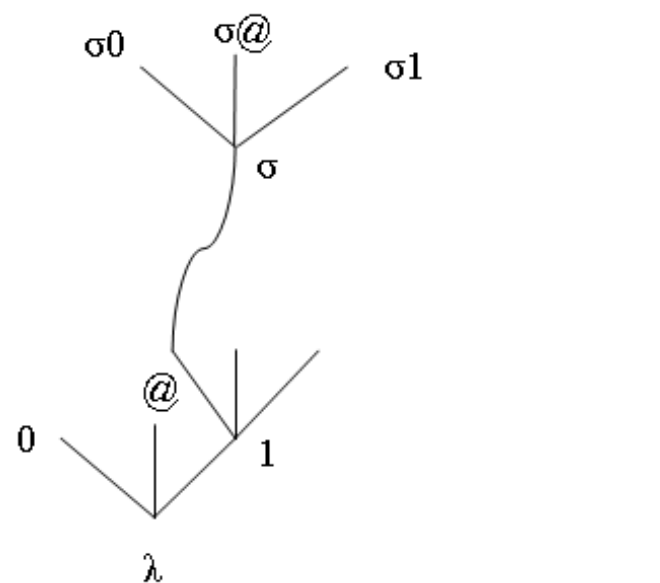

Figure 4.1: $2^{<\omega} \cup 2^{<\omega} @$

Let $2^{<\omega} @$ be the set of symbols $\sigma @$ with $\sigma$ a binary string. Define a lexicographical order $\leq_{l e x}$ on $2^{<\omega} \cup 2^{<\omega} @$ based on $0<_{l e x} @<_{\text {lex }} 1$. In particular, for each $\sigma \in 2^{<\omega}, \sigma 0<_{\text {lex }} \sigma @<_{\text {lex }} \sigma 1$. We can view $2^{<\omega} \cup 2^{<\omega} @$ as a ternary tree, where each node $\sigma \in 2^{<\omega}$ has three immediate extensions, namely $\sigma 0<_{\text {lex }} \sigma @<_{\text {lex }} \sigma 1$, while each $\sigma @$ with $\sigma \in 2^{<\omega}$ has no extensions. The ternary tree is roughly pictured in Figure 4.1.

Now define a length-lexicographical order, say $\leq_{l e n-l e x}$, on $2^{<\omega @}$ based on the lexicographical order on $2^{<\omega} @$. That is, for $\sigma @$ and $\tau @$ in $2^{<\omega} @$,

$$
\sigma @ \leq_{\text {len-lex }} \tau @ \Leftrightarrow|\sigma|<|\tau| \vee\left(|\sigma|=|\tau| \wedge \sigma @ \leq_{\text {lex }} \tau @\right) .
$$




\subsection{The $\mathcal{P}$-strategy}

We will build a computable group $\mathscr{G}$ stage by stage such that $\mathscr{G}$ is isomorphic to $\mathbb{Q}^{\infty}$ via a $\Delta_{2}^{0}$-isomorphism. During the construction, we maintain a finite set $B_{s}$ at each stage $s$ which serves as an approximation of an infinite basis $B$ of $\mathscr{G}$. Then

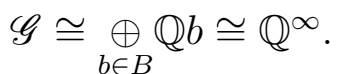

Now we start to define $\mathscr{G}$. For each $\sigma @ \in 2^{<\omega} @$, we introduce a base-element $q_{\sigma} @$ for $\mathscr{G} . q_{\sigma} @$ will be enumerated into the constructed group at stage $|\sigma| \cdot q_{\sigma} @$ has two totally different fates.

(1) If the $\mathcal{R}$-strategy adds a dependence relation of the form $q_{\sigma @}=r q_{\tau} @$ for some

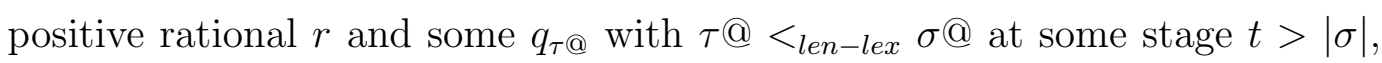
then $q_{\sigma} @$ is said to be non-active at stage $t$. In this case, $q_{\sigma} @ B_{t^{\prime}}$ for all $t^{\prime} \geq t$

(2) If no dependence relations of the form $q_{\sigma @}=r q_{\tau} @$ are added during stages in $[|\sigma|, t], q_{\sigma} @$ is said to be active at stage $t$. In this case, $q_{\sigma @} \in B_{t}$.

Hence, either $q_{\sigma} @$ is active forever in which case $q_{\sigma @} \in B$, or $q_{\sigma} @$ becomes non-active at some stage $>|\sigma|$ in which case $q_{\sigma} \notin B$.

Let $\mathscr{H}$ be the computable additive group $\underset{\sigma \in 2^{<\omega}}{\oplus} \mathbb{Q} q_{\sigma} @$. Elements of $\mathscr{H}$ are just finite $\mathbb{Q}$-linear sums of elements in $\left\{q_{\sigma} @ \sigma \in 2^{<\omega}\right\}$. The constructed group $\mathscr{G}$ is indeed a quotient group of $\mathscr{H}$.

Fix a computable enumeration $\mathscr{H}_{s}$ of $\mathscr{H}$ such that at most one element is enumerated into $\mathscr{H}_{s}$ at stage $s$, and for each nonzero $x_{0} q_{\sigma_{0} @}+\cdots+x_{m} q_{\sigma_{m}} @$ in $\mathscr{H}_{s}$, it satisfies $x_{m} \neq 0$, and for each $i \leq m,\left|\sigma_{i}\right| \leq s$. Assume that $\mathscr{H}_{0}=\left\{0_{\mathscr{H}}\right\}$.

Construction of $\mathscr{G}=\left(G,+, 0_{\mathscr{G}}\right)$.

At stage 0 .

$G_{0}=\left\{0_{\mathscr{G}}, q_{@}\right\}$, where $0_{\mathscr{G}}$ is the zero element of $\mathscr{G}$ and $q_{@}$ is the active baseelement at stage $0 . B_{0}=\left\{q_{@}\right\}$.

At the end of stage $s, G_{s}$ is the set containing all elements of $\mathscr{G}$ enumerated by stage $s . B_{s}$ is the set of active base-elements at stage $s$. Each nonzero $g \in G_{s}$ can 
be expressed as a unique $\mathbb{Q}$-linear sum of the form

$$
r_{0} q_{\sigma_{0} @}+\cdots+r_{n} q_{\sigma_{n} @}
$$

of active base-elements at stage $s$ with $r_{n} \neq 0$ and $\sigma_{0} @<_{\text {lex }} \sigma_{1} @ \cdots<_{l e x} \sigma_{n} @$ (this restriction is clear in next paragraph about computable orders). By convention, the unique $\mathbb{Q}$-linear sum of $0_{\mathscr{G}}$ is the empty sum.

We require $\sigma_{0} @<_{l e x} \sigma_{1} @ \cdots<_{l e x} \sigma_{n} @$ in the expression $g=r_{0} q_{\sigma_{0}} @ \cdots+r_{n} q_{\sigma_{n}} @$ because we build the computable order $\leq_{\mathscr{G}}$ such that the sign of $g$ under $\leq_{\mathscr{G}}$ is just the sign of the nonzero $r_{m}$ with $m$ least if such a $r_{m}$ exists, and 0 otherwise. That is, $\leq_{\mathscr{G}}$ is indeed a kind of lexicographical order (the construction of $\leq_{\mathscr{G}}$ is clear in the following $\mathcal{Q}$-strategy).

At stage $s+1$.

Define domain $G_{s+1}$. There are two cases depending on whether the $\mathcal{R}$-strategy adds dependence relations.

Case 1. If no dependence relations are added among active base-elements in $B_{s}$ by the $\mathcal{R}$-strategy at stage $s+1$, then each base-element in $B_{s}$ is still active at stage $s+1$. For each $\sigma$ with length $s+1$, add a new base-element $q_{\sigma} @$ into $B_{s+1}$. That is,

$$
B_{s+1}=B_{s} \cup\left\{q_{\sigma} @|\sigma|=s+1\right\} .
$$

If $\mathscr{H}_{s+1}=\mathscr{H}_{s}$, let $G_{s+1}=G_{s} \cup B_{s+1}$. If there is an element, $x_{0} q_{\sigma_{0} @}+\cdots+x_{m} q_{\sigma_{m} @}$ say, in $\mathscr{H}_{s+1}-\mathscr{H}_{s}$. Note that $x_{m} \neq 0$, and for each $i \leq m,\left|\sigma_{i}\right| \leq s+1$.

(1) If there are $q_{\sigma_{i} @ \mathrm{~S}}$ such that $q_{\sigma_{i} @}$ is non-active at stage $s+1$, just update the $\mathbb{Q}$-linear sum by replacing such $q_{\sigma_{i} @ \text { S }}$ by active base-elements at stage $s+1$ with corresponding scaling.

(2) Otherwise, each $q_{\sigma_{i}} @$ in the $\mathbb{Q}$-linear sum is active at stage $s+1$.

In both cases, the $\mathbb{Q}$-linear sum has a new expression, say $a_{0} q_{\tau_{0} @}+\cdots+a_{n} q_{\tau_{n} @}$, as a $\mathbb{Q}$-linear sum of elements in $B_{s+1}$. If all $a_{i}$ with $i \leq n$ are 0 , then the $\mathbb{Q}$-linear sum is indeed the zero element of $\mathscr{G}$, just let $G_{s+1}=G_{s} \cup B_{s+1}$. Otherwise, find the largest $m \leq n$ with $a_{m} \neq 0$, there are two cases: 
(1) If $a_{0} q_{\tau_{0} @}+\cdots+a_{m} q_{\tau_{m}} @$ is not an expression of elements enumerated into $\mathscr{G}$ before, then let $G_{s+1}=G_{s} \cup B_{s+1} \cup\left\{a_{0} q_{\tau_{0} @}+\cdots+a_{m} q_{\tau_{m} @}\right\}$.

(2) Otherwise, let $G_{s+1}=G_{s} \cup B_{s+1}$.

Case 2. If there are two active base-elements, say $q_{\sigma @}, q_{\tau} @$ in $B_{s}$, such that the pair $(\sigma @, \tau @)$ is enumerated into link at stage $s+1$, i.e., the relation $\operatorname{link}(\sigma @, \tau @)$ holds at stage $s+1$ [here, link is a c.e. equivalence relation on $2^{<\omega} @$ which will be defined in the basic $\mathcal{R}$-strategy].

We will add dependence relations of the form $q_{\eta @}=r q_{\rho @ \text { with } r>0, \rho @<_{\text {len-lex }}}$ $\eta @$, i.e., $|\rho @|<|\eta @|$ or $|\rho @|=|\eta @|$ with $\rho @<_{\text {lex }} \eta @$ (the method for adding dependence relations will be provided in the following $\mathcal{R}$-strategy). In this case, $q_{\eta} @$ is non-active at stage $s+1$. Once such a dependence relation is added, we update the expression of elements in $G_{s}$. The added relations ensure that for any $g, h \in G_{s}$, if the expression of $g, h$ at stage $s$ is different, then the new expression of $g, h$ at stage $s+1$ is still different.

Now add new active base-elements $q_{\sigma} @$ with $|\sigma|=s+1$ at stage $s+1$. Let $B_{s+1}$ be the set of active base-elements at stage $s+1$ (the concert definition of $B_{s+1}$ will be clear in the following $\mathcal{R}$-strategy).

If there is a new $x$ enumerating into $\mathscr{H}_{s+1}$, first update $x$ as a $\mathbb{Q}$-linear sum of active base-elements at stage $s+1$, and then add this $\mathbb{Q}$-linear sum into $G_{s+1}$ as in case 1.

This ends the construction of $G_{s+1}$.

Define the partial addition $+_{s+1}$ on $G_{s+1}$. Note that each element of $G_{s+1}$ has a unique $\mathbb{Q}$-linear sum of active base-elements in $B_{s+1}$. For $x, y, z \in G_{s+1}$, if the addition of the unique $\mathbb{Q}$-linear sum of $x$ and $y$ is the same as that of $z$, then let $x+{ }_{s+1} y=z$.

If we already defined $x+{ }_{s} y=z$ at stage $s$, then $x+_{s+1} y=z$. Indeed, after substituting one dependence relation of the form $q_{\eta @}=r q_{\rho}$ into the old expression of $x, y, z$, we obtain the new expression of them as a unique $\mathbb{Q}$-linear sum of current active base-elements respectively. It is a direct checking that the new expression 
of them also satisfies the condition that the addition of the new expression of $x, y$ equals that of $z$.

This ends the construction of $+_{s+1}$.

\subsection{The $\mathcal{Q}$-strategy}

Due to the one-to-one correspondence between orders and positive cones on computable groups, when building an order on a group, it is enough to specify the set of positive elements or alternatively to specify the sign of elements in the group. We build $\leq_{\mathscr{G}}$ by specifying the sign of elements in $\mathscr{G}$ under $\leq_{\mathscr{G}}$. That is, for each $g \in \mathscr{G}$, the sign of $g$ is

$$
\operatorname{sign}_{\leq_{\mathscr{G}}}(g):= \begin{cases}+1, & \text { if } g>\mathscr{G} 0_{\mathscr{G}} \\ 0, & \text { if } g=0_{\mathscr{G}} \\ -1, & \text { if } g<\mathscr{G} 0_{\mathscr{G}}\end{cases}
$$

Then the positive cone of $\leq_{\mathscr{G}}$ is just $\left\{g \in G: \operatorname{sign}_{\leq_{\mathscr{G}}}(g)=+1\right\} \cup\left\{0_{\mathscr{G}}\right\}$.

At each stage $s$, we approximate $\leq_{\mathscr{G}}$ through a type of lexicographical order on $G_{s}$ in the following sense. The sign of $0_{\mathscr{G}}$ is always 0 . Now assign the sign of nonzero elements in $G_{s}$. First, each nonzero element $g \in G_{s}$ has a unique $\mathbb{Q}$-linear sum of active base-elements of the form

$$
r_{0} q_{\sigma_{0} @}+r_{1} q_{\sigma_{1} @}+\cdots+r_{n} q_{\sigma_{n} @}
$$

with $r_{n} \neq 0$ and $\sigma_{0} @<_{\text {lex }} \sigma_{1} @ \cdots<_{\text {lex }} \sigma_{n} @$. Second, for each such a nonzero $g$, find the least $m$ with $r_{m} \neq 0$ in the expression, and then define the sign of $g$ to be the usual sign of $r_{m}$ as rational numbers (i.e., for $r \in \mathbb{Q}$, if $r>0$, it has sign +1 ; if $r=0$, it has sign 0 ; if $r<0$, it has sign -1 ). During the construction, we maintain the sign of elements unchanged.

Construction of $\operatorname{sign}_{\leq \mathscr{G}}$.

At stage 0 .

$G_{0}=\left\{0_{\mathscr{G}}, q_{@}\right\}$. Let the sign of $0_{\mathscr{G}}$ be 0 and the sign of $q_{@}$ be +1 .

At the end of stage $s$, we have already appointed the sign of elements in $G_{s}$. Now define the sign of elements in $G_{s+1}$. 
At stage $s+1$.

Case 1. If no dependence relations are added via the $\mathcal{R}$-strategy, then the set of active base-elements at stage $s+1$ is $B_{s} \cup\left\{q_{\sigma @}:|\sigma|=s+1\right\}$. The sign of elements in $G_{s}$ is unchanged at stage $s+1$.

For each new added active base-element $q_{\sigma @}$ with $|\sigma|=s+1$, let $\operatorname{sign}_{\leq_{\mathscr{G}}}\left(q_{\sigma @}\right)=$ +1 . If there is one more added element, $x$ say, in $G_{s+1}$. Then $x$ is of the form $a_{0} q_{\sigma_{0} @}+a_{1} q_{\sigma_{1} @}+\cdots+a_{m} q_{\sigma_{m} @}$ with $a_{m} \neq 0$ and $\sigma_{0} @<_{l e x} \sigma_{1} @ \cdots<_{l e x} \sigma_{n} @$ as a unique $\mathbb{Q}$-linear sum of active base-elements at stage $s+1$. Find the least $i \leq m$ such that $a_{i} \neq 0$, and then let $\operatorname{sign}_{\leq \mathscr{G}}(x)$ be +1 if $a_{i}$ is positive, and -1 otherwise.

Case 2. If there are two active base-elements, say $q_{\sigma @}, q_{\tau} @$ in $B_{s}$, such that the pair $(\sigma @, \tau @)$ is enumerated into link at stage $s+1$, i.e., the relation $\operatorname{link}(\sigma @, \tau @)$ holds at stage $s+1$, we will add dependence relations to satisfy the $\mathcal{R}$-strategy. To make $\operatorname{sign}_{\leq \mathscr{G}}$ computable, we will ensure that such dependence relations added at stage $s+1$ will not change the sign of elements in $G_{s}$.

Without loss of generality, let $\sigma @<_{\text {lex }} \tau @$. In order to preserve signs of elements in $G_{s}$, the $\mathcal{R}$-strategy first finds all $q_{\eta @} \in B_{s}$ such that

(1) $\sigma @ \leq_{\text {lex }} \eta @ \leq_{\text {lex }} \tau @$;

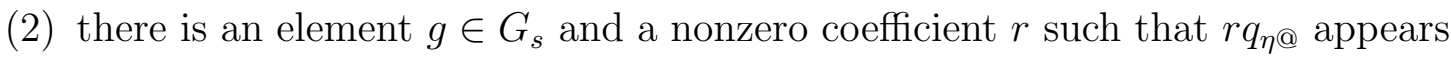
in the unique expression of $g$ as a $\mathbb{Q}$-linear sum of elements in $B_{s}$.

Now list all such $\eta @_{\mathrm{s}}$ under the lexicographical order as

$$
\sigma @=\xi_{0} @<_{l e x} \xi_{1} @ \cdots<_{l e x} \xi_{n-1} @<_{l e x} \xi_{n} @=\tau @ .
$$

By the definition of $\operatorname{link}, \operatorname{link}(\sigma @, \tau @)$ holds implies for all $i, j \leq n, \operatorname{link}\left(\xi_{i} @, \xi_{j} @\right)$ holds.

In the following $\mathcal{R}$-strategy, we will add dependence relations of the form

$$
q_{\eta @}=r q_{\rho @}
$$

with proper $r>0$ and $\rho @<_{\text {len-lex }} \eta @$, i.e., $|\rho|<|\eta|$ or $\left(|\rho|=|\eta| \wedge \rho @<_{\text {lex }} \eta @\right)$.

We start with $q_{\xi_{0} @}$ and $q_{\xi_{1} @}$. Now each $g \in G_{s}$ has an expression of the form

$$
g=\cdots+r_{\xi_{0}} q_{\xi_{0} @}+r_{\xi_{1}} q_{\xi_{1} @}+\cdots .
$$


Let

$$
\delta_{0} @:= \begin{cases}\xi_{0} @, & \text { if } \xi_{0} @<_{l e n-l e x} \xi_{1} @ \\ \xi_{1} @, & \text { if } \xi_{1} @<_{l e n-l e x} \xi_{0} @\end{cases}
$$

and $\gamma_{0} @ \in\left\{\xi_{0} @, \xi_{1} @\right\}-\left\{\delta_{0} @\right\}$. We first add relations of the form

$$
q_{\gamma_{0} @}=c_{0} q_{\delta_{0} @}
$$

with proper $c_{0}>0$ such that the sign of elements in $G_{s}$ does not change after substituting this relation.

When $\xi_{0} @<_{\text {len-lex }} \xi_{1} @$, we add $q_{\xi_{1} @}=c_{0} q_{\xi_{0} @}$. Then $g$ has a new expression

$$
\cdots+\left(r_{\xi_{0}}+r_{\xi_{1}} c_{0}\right) q_{\xi_{0} @}+0 q_{\xi_{1} @}+\cdots .
$$

(1) If $r_{\xi_{0}}=0$, then the sign of $r_{\xi_{1}}$ equals the sign of $r_{\xi_{1}} c_{0}$.

(2) If $r_{\xi_{0}} \neq 0$, then we will choose $c_{0}$ such that the sign of $r_{\xi_{0}}$ equals the sign of $r_{\xi_{0}}+r_{\xi_{1}} c_{0}$.

Then the sign of $g$ computed according to the new expression is the same as before.

When $\xi_{1} @<_{\text {len-lex }} \xi_{0} @$, we add $q_{\xi_{0} @}=c_{0} q_{\xi_{1}} @$. Now $g$ has a new expression

$$
\cdots+0 q_{\xi_{0} @}+\left(r_{\xi_{0}} c_{0}+r_{\xi_{1}}\right) q_{\xi_{1} @}+\cdots .
$$

(1) If $r_{\xi_{0}}=0$, then the expression of $g$ does not change.

(2) If $r_{\xi_{0}} \neq 0$, then we will choose $c_{0}$ such that the sign of $r_{\xi_{0}}$ equals the sign of $r_{\xi_{0}} c_{0}+r_{\xi_{1}}$.

So the sign of $g$ does not change after substituting the relation.

Now each element in $G_{s}$ has a unique expression as a $\mathbb{Q}$-linear sum of elements

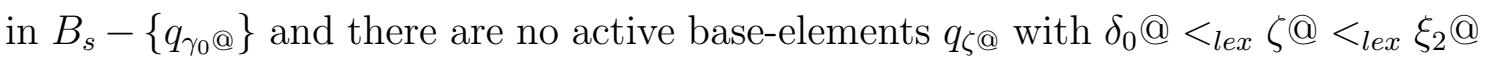
in the expression.

We then deal with $q_{\delta_{0} @}$ and $q_{\xi_{2} @}$ in a similar manner as dealing with $q_{\xi_{0} @}$ and

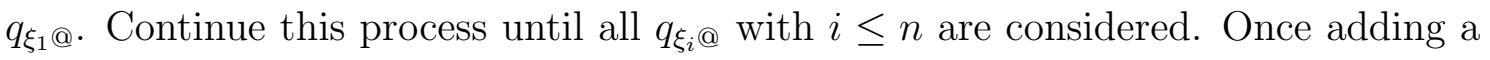
dependence relation, we preserve the sign of elements in $G_{s}$. So the whole process does not change the sign of elements in $G_{s}$. 
In general, for $1 \leq i \leq n-1$, define by induction that $\delta_{i} @$ is the lengthlexicographically smaller element in $\left\{\delta_{i-1} @, \xi_{i+1} @\right\}$ and that $\gamma_{i} @$ is the other element in $\left\{\delta_{i-1} @, \xi_{i+1} @\right\}$.

Now the set of active base-elements is

$$
B_{s+1}=\left(B_{s}-\left\{q_{\gamma_{i} @}: 0 \leq i \leq n-1\right\}\right) \cup\left\{q_{\sigma @}:|\sigma|=s+1\right\} .
$$

The sign of each new active base-element $q_{\sigma} @$ is +1 , and the sign of the new added element (if such an element exists) is determined as in case 1.

\subsection{The $\mathcal{R}$-strategy}

The $\mathcal{R}$-requirement says that for any set $A$,

(1) if the constructed group $\mathscr{G}$ has an $A$-computable order, say $\leq^{A}$, with $\leq^{A} \neq \leq \mathscr{G}$ and $\leq^{A} \neq \leq_{\mathscr{G}}^{*}$, then $A$ is PA-complete;

(2) if $A$ is PA-complete, then $\mathscr{G}$ has an $A$-computable order which is neither $\leq_{\mathscr{G}}$ nor $\leq_{\mathscr{G}}^{*}$.

\subsubsection{Background on PA-complete sets}

We will use an equivalent definition for a set being PA-complete that $A$ is PAcomplete iff it computes a $\{0,1\}$-valued diagonally nonrecursive function, i.e., a $\{0,1\}$-valued function $f$ such that for all $x$, if $\varphi_{x}(x) \downarrow$, then $f(x) \neq \varphi_{x}(x)$.

For a binary tree $T$, an infinite binary string $f$ is an infinite path on $T$ if for all $n$, its prefix $f(0) f(1) \cdots f(n)$, denoted by $f \uparrow_{n+1}$, is in $T$; set $f \uparrow_{0}:=\lambda$, the empty string. We view $f$ as a $\{0,1\}$-valued function (i.e., the characteristic function of $\{n \in \mathbb{N}: f(n)=1\})$. We use $[T]$ to denote the set of infinite paths of $T$.

One can build a co-c.e. binary tree $T$ (i.e., the complement of $T$ is c.e.) such that $[T]$ is just the set of $\{0,1\}$-valued diagonally nonrecursive functions. Then for a set $A, A$ is PA-complete iff it computes an infinite path of $T$. Now based on this $T$, to satisfy $\mathcal{R}$, we only need to meet the following $\mathcal{R}^{\prime}$ :

$\mathcal{R}^{\prime}$ : For any set $A$, if $\leq^{A}$ is an $A$-computable order on $\mathscr{G}$ which is neither $\leq \mathscr{G}$ nor $\leq_{\mathscr{G}}^{*}$, then $A$ computes an infinite path on $T$. If $f \in[T]$, then $f$ computes an order on $\mathscr{G}$ which is not computable. 
We now construct the co-c.e. tree $T$ by stages.

The construction of $T$.

At stage 0. $T_{0}=2^{<\omega}$, the full binary tree. $F_{0}=\varnothing$, the complement of $T_{0}$.

At the end of stage $s$, we already obtained approximations $T_{s}$ and $F_{s}$ such that $T_{s} \cup F_{s}=2^{<\omega}$.

At stage $s+1$. For each $\sigma \in T_{s}$ of length $\leq s+1$, if there is a $x<|\sigma|$ such that $\varphi_{x, s+1}(x)$ is defined with value $\sigma(x)$, then enumerate $\sigma$ into $F_{s+1}$. Let $T_{s+1}=$ $2^{<\omega}-F_{s+1}$.

This ends the construction of $T$ at stage $s+1$.

Clearly, $T=\bigcap_{s \in \mathbb{N}} T_{s}$ is a co-c.e. set. For any binary string $\sigma, \sigma \in T$ iff it satisfies $\forall x<|\sigma|\left[\varphi_{x}(x) \downarrow \rightarrow \varphi_{x}(x) \neq \sigma(x)\right]$, then any initial segment of $\sigma$ is also in $T$. So $T$ is a tree. Furthermore, $f \in[T]$ iff $f$ is a $\{0,1\}$-valued diagonally nonrecursive function.

By using infinite paths on $T$, we will define an equivalence relation on $2^{<\omega} @$ (recall that $2^{<\omega} @=\left\{\sigma @: \sigma \in 2^{<\omega}\right\}$ ). The lexicographical order $\leq_{\text {lex }}$ on $2^{<\omega} \cup 2^{<\omega} @$ is already defined in section 4.2 , where for any binary string $\sigma, \sigma 0<_{\text {lex }} \sigma @<_{\text {lex }} \sigma 1$.

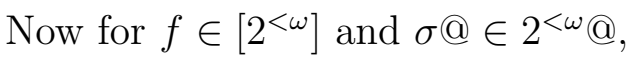

- say that $f$ is lexicographically less than $\sigma @$, denoted by $f<_{\text {lex }} \sigma @$, if there is an $i<|\sigma|+1$ such that $f \uparrow_{i}=\sigma @ \Upsilon_{i}$ and $f(i)<_{\text {lex }} \sigma @(i)$; and $\sigma @$ is lexicographically less than $f$, denoted by $\sigma @<_{l e x} f$, otherwise.

Hence, if $f<_{l e x} \sigma @$, then for all $n, f \uparrow_{n}<_{l e x} \sigma @$; otherwise, there is a $m$ such that for all $n \geq m, \sigma @<_{\text {lex }} f \uparrow_{n}$.

Definition 4.1 Let $T$ be the co-c.e. tree, where $[T]$ is the set of $\{0,1\}$-valued diagonally nonrecursive functions. For $\sigma @, \tau @ \in 2^{<\omega} @, \operatorname{link}(\sigma @, \tau @)$ holds if there is no $f \in[T]$ such that $f$ is lexicographically between $\sigma @$ and $\tau @$.

We use link to denote the set of pairs $(\sigma @, \tau @)$ such that $\operatorname{link}(\sigma @, \tau @)$ holds. One can directly check that link is an equivalence relation on $2^{<\omega} @$.

Proposition 4.2 link is a c.e. equivalence relation. 
Proof: Fix a computable approximation $T_{s}$ of $T$ (as in the construction of $T$ above) such that $T_{s+1} \subseteq T_{s}$ and $T=\bigcap_{s \in \mathbb{N}} T_{s}$.

For $\sigma @, \tau @ \in 2^{<\omega} @$, assume that $\sigma @<_{\text {lex }} \tau @$. link $(\sigma @, \tau @)$ holds iff $\left\{\eta \in 2^{<\omega}: \sigma @<_{\text {lex }} \eta<_{\text {lex }} \tau @\right\} \cap T$ is finite iff

$$
\exists n \exists s \forall \rho \in 2^{<\omega}\left[\left(|\rho|=n \wedge \sigma @<_{\text {lex }} \rho<_{\text {lex }} \tau @\right) \rightarrow \rho \notin T_{s}\right] .
$$

So link is $\Sigma_{1}^{0}$.

\subsubsection{The $\mathcal{R}^{\prime}$-strategy}

The $\mathcal{R}^{\prime}$-requirement says that:

(1) for any set $A$, if $\leq^{A}$ is an $A$-computable order on $\mathscr{G}$ which is neither $\leq_{\mathscr{G}}$ nor $\leq_{\mathscr{G}}^{*}$, then $A$ computes an infinite path on $T$;

(2) if $f \in[T]$, then $f$ computes an order on $\mathscr{G}$ which is not computable.

In order to satisfy (1) of the $\mathcal{R}^{\prime}$-requirement, we will initiate an $A$-effective process for searching nodes in $2^{<\omega} @$ which approximates an infinite path on $T$ lexicographically form below and above. Our method is to add dependence relations of the form $q_{\eta @}=r q_{\rho @}$, where $r>0, \operatorname{link}(\eta @, \rho @)$ holds, and $\rho @<_{\text {len-lex }} \eta @$.

In this section, we will develop the method for adding dependence relations. We will show that this method works (i.e., the $\mathcal{R}^{\prime}$-requirement is satisfied) in sections 4.6.3 and 4.6.4.

Since the length-lexicographical order on $2^{<\omega} @$ is a well ordering, for each $\tau @ \in$

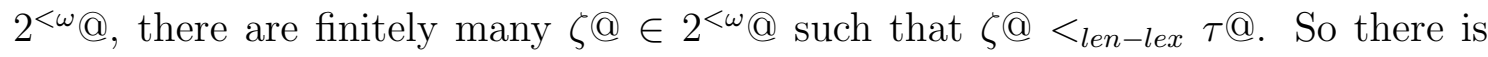
a least $\zeta @ \leq_{\text {len-lex }} \tau @$ such that $\zeta @$ and $\tau @$ are linked, where the least is taking

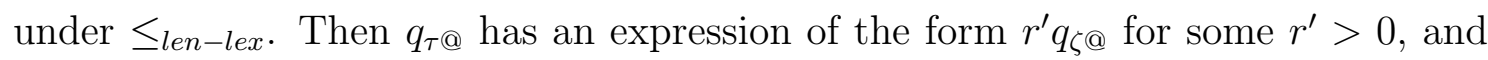
then no new expressions are assigned to $q_{\tau} @$. Since an element, $x$ say, is enumerated into $G_{s}$ for first time is always of the form

$$
x_{0} q_{\sigma_{0} @}+\cdots+x_{m} q_{\sigma_{m}} @
$$

of a $\mathbb{Q}$-linear sum of active base-elements at stage $s$, there is a large enough stage after which no more expressions are assigned to all $q_{\sigma_{i} @}$ with $0 \leq i \leq m$. Then $x$ will arrive at a final expression as a $\mathbb{Q}$-linear sum of active base-elements. 
When adding $q_{\eta @}=r q_{\rho @}$ at stage $s+1$, we need to ensure the following two conditions:

(1) the assignment of $\mathbb{Q}$-linear sums to elements in $G_{s}$ remains one-to-one (i.e., the dependence relation is compatible with the $\mathcal{P}$-strategy of constructing $\mathscr{G}$ );

(2) the sign of elements in $G_{s}$ does not change (i.e., the dependence relation is compatible with the $\mathcal{Q}$-strategy of constructing $\left.\leq_{\mathscr{G}}\right)$.

\section{Adding dependence relations.}

Since link is a c.e. equivalence relation on $2^{<\omega} @$, just fix a computable enumeration $l_{i n k}$ of link such that at each stage $s$ there is at most one pair of the form $(\sigma @, \tau @)$ with $|\sigma|,|\tau|<s$ enumerating into link .

At stage $0 . \operatorname{link}_{0}=\varnothing . q_{@}$ is the only active base-element at stage 0 .

Assume that we have already obtained $B_{s}$, the set of active base-elements at stage $s$. Each element in $B_{s}$ is of the form $q_{\tau} @$ with $|\tau| \leq s$. Each $g \in G_{s}$ has a unique $\mathbb{Q}$-linear sum of elements in $B_{s}$. By convention, the unique $\mathbb{Q}$-linear sum for $0_{\mathscr{G}}$ is just the empty sum.

At stage $s+1$.

Case 1. If $l i n k_{s}=l i n k_{s+1}$, or $(\sigma @, \tau @) \in l i n k_{s+1}-l i n k_{s}$, where at least one of $q_{\sigma} @$ and $q_{\tau} @$ is non-active at stage $s$, then no dependence relations are added at stage $s+1$. Just let $B_{s+1}=B_{s} \cup\left\{q_{\sigma @}:|\sigma|=s+1\right\}$.

Case 2. Suppose that there is a pair $(\sigma @, \tau @)$ of active base-elements at stage $s$ enumerating into $l i n k_{s+1}$. We will add dependence relations between $q_{\sigma} @$ and $q_{\tau} @$. Without loss of generality, we assume that $\sigma @<_{\text {lex }} \tau @$.

First, find all $q_{\eta @} \in B_{s}$ such that

(1) $\sigma @ \leq_{\text {lex }} \eta @ \leq_{\text {lex }} \tau @$;

(2) there is an element $g \in G_{s}$ and a nonzero coefficient $r$ such that $r q_{\eta @}$ appears in the unique expression of $g$ as a $\mathbb{Q}$-linear sum of elements in $B_{s}$. 
Second, list all such $\eta @_{\mathrm{s}}$ under the lexicographical order as follows:

$$
\sigma @=\xi_{0} @<_{l e x} \xi_{1} @ \cdots<_{l e x} \xi_{n-1} @<_{l e x} \xi_{n} @=\tau @ .
$$

Since $\operatorname{link}(\sigma @, \tau @)$ holds, for all $i, j \leq n, \operatorname{link}\left(\xi_{i} @, \xi_{j} @\right)$ holds.

We start to add dependence relations between $q_{\xi_{0} @}$ and $q_{\xi_{1}} @$. Now each element

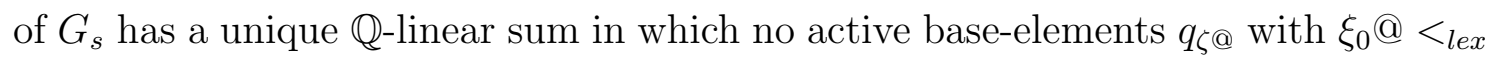
$\zeta @<_{\text {lex }} \xi_{1} @$ appears. That is, each $g \in G_{s}$ has a unique expression of the form $g=\cdots+r_{\xi_{0}} q_{\xi_{0}} @+r_{\xi_{1}} q_{\xi_{1} @}+\cdots$.

Let $\delta_{0} @$ be the length-lexicographically less element between $\xi_{0} @$ and $\xi_{1} @$, and $\gamma_{0} @$ be the other element. In module $\mathrm{D}(0)$, we will add a relation of the form $q_{\gamma_{0} @}=c_{0} q_{\delta_{0}} @$ with proper $c_{0}>0$ such that after substituting this relation, the new expressions of elements in $G_{s}$ as $\mathbb{Q}$-linear sums of elements in $B_{s}-\left\{q_{\gamma_{0}} @\right.$ remain one-to-one and the signs of elements in $G_{s}$ computed according to new expressions remain unchanged.

Module $\mathrm{D}(0)$.

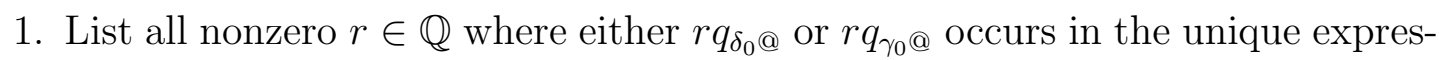
sion of an element in $G_{s}$ as a $\mathbb{Q}$-linear combination of active base-elements at stage $s$ as follows:

$$
r_{1}, r_{2}, \cdots, r_{l}
$$

2. Set $r_{0}:=0$. Choose a large enough $k \in \mathbb{N}^{+}$such that for all $i, i^{\prime}, j, j^{\prime} \leq l$ with $r_{j}-r_{j^{\prime}} \neq 0$,

$$
k>\frac{\left|r_{i}-r_{i^{\prime}}\right|}{\left|r_{j}-r_{j^{\prime}}\right|}
$$

2.1. If $\delta_{0} @<_{\text {lex }} \gamma_{0} @$, each element, say $g \in G_{s}$, has an expression of the form

$$
g=\cdots+r_{\delta_{0}} q_{\delta_{0} @}+r_{\gamma_{0}} q_{\gamma_{0} @}+\cdots .
$$

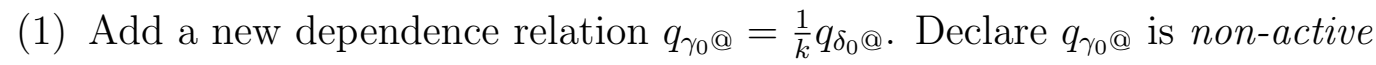
at stage $s+1$. 
(2) We now show that adding $q_{\gamma_{0} @}=\frac{1}{k} q_{\delta_{0} @}$ does not change the sign of elements in $G_{s}$.

By substituting $q_{\gamma_{0} @}=\frac{1}{k} q_{\delta_{0}} @ g$ has a new expression

$$
g=\cdots+\left(r_{\delta_{0}}+\frac{r_{\gamma_{0}}}{k}\right) q_{\delta_{0} @}+0 q_{\gamma_{0} @}+\cdots .
$$

When $r_{\delta_{0}}=0$. Since $k$ is positive, the sign of $r_{\gamma_{0}}$ equals the sign of $\frac{r_{\gamma_{0}}}{k}$.

When $r_{\delta_{0}} \neq 0$. We show that the sign of $r_{\delta_{0}}$ equals the sign of $r_{\delta_{0}}+\frac{r_{\gamma_{0}}}{k}$.

(i) $r_{\delta_{0}}>0$. Since $k>0$, if $r_{\gamma_{0}} \geq 0$, then $r_{\delta_{0}}+\frac{r_{\gamma_{0}}}{k}>0$. If $r_{\gamma_{0}}<0$, by the choice of $k, k>\frac{-r_{\gamma_{0}}}{r_{\delta_{0}}}$, that is, $r_{\delta_{0}}+\frac{r_{\gamma_{0}}}{k}>0$.

(ii) $r_{\delta_{0}}<0$. Since $k>0$, if $r_{\gamma_{0}} \leq 0$, then $r_{\delta_{0}}+\frac{r_{\gamma_{0}}}{k}<0$. If $r_{\gamma_{0}}>0$, since $k>\frac{r_{\gamma_{0}}}{-r_{\delta_{0}}}, r_{\delta_{0}}+\frac{r_{\gamma_{0}}}{k}<0$.

By definition, the current sign of nonzero $g \in G_{s}$ is the sign of the first nonzero rational coefficient in this new expression as a $\mathbb{Q}$-linear sum of current active base-elements. Hence, after adding the dependence relation $q_{\gamma_{0} @}=\frac{1}{k} q_{\delta_{0} @}$, the current sign of $g \in G_{s}$ is the same as before.

(3) We now show that adding $q_{\gamma_{0} @}=\frac{1}{k} q_{\delta_{0} @}$ ensures that the assignment of $\mathbb{Q}$-linear sums to elements in $G_{s}$ remains one-to-one.

For $x, x^{\prime} \in G_{s}$, suppose that at stage $s, x=\cdots+a_{\delta_{0}} q_{\delta_{0} @}+a_{\gamma_{0}} q_{\gamma_{0} @}+\cdots$, and $x^{\prime}=\cdots+a_{\delta_{0}}^{\prime} q_{\delta_{0} @}+a_{\gamma_{0}}^{\prime} q_{\gamma_{0} @}+\cdots$. If the new expression of $x$ and $x^{\prime}$ is the same, then the coefficient of active base-elements at stage $s$ other than $q_{\delta_{0}} @ q_{\gamma_{0} @ \text { is the same and }}$

$$
a_{\delta_{0}}+\frac{a_{\gamma_{0}}}{k}=a_{\delta_{0}}^{\prime}+\frac{a_{\gamma_{0}}^{\prime}}{k}
$$

i.e., $\left(a_{\delta_{0}}-a_{\delta_{0}}^{\prime}\right) k=\left(a_{\gamma_{0}}^{\prime}-a_{\gamma_{0}}\right)$.

(i) If $a_{\delta_{0}}=a_{\delta_{0}}^{\prime}$, then $a_{\gamma_{0}}^{\prime}=a_{\gamma_{0}}$. In this case, the old expression of $x, x^{\prime}$ at stage $s$ is the same. 
(ii) If $a_{\delta_{0}} \neq a_{\delta_{0}}^{\prime}$, then $k=\frac{a_{\gamma_{0}}^{\prime}-a_{\gamma_{0}}}{a_{\delta_{0}}-a_{\delta_{0}}^{\prime}}$. In this case, the old expression of $x, x^{\prime}$ at stage $s$ is different. However, by the choice of $k, k>\frac{\left|a_{\gamma_{0}}^{\prime}-a_{\gamma_{0}}\right|}{\mid a_{\delta_{0}}-a_{\delta_{0}}^{\prime}} \cdot$. So this case is impossible.

Hence, if $x, x^{\prime} \in G_{s}$ have different expression as $\mathbb{Q}$-linear sums of active base-elements at stage $s$, then the new expression of them as $\mathbb{Q}$-linear sums of current active base-elements is still different.

2.2. If $\gamma_{0} @<_{\text {lex }} \delta_{0} @$, each element, say $g \in G_{s}$, has an expression of the form

$$
g=\cdots+r_{\gamma_{0}} q_{\gamma_{0} @}+r_{\delta_{0}} q_{\delta_{0} @}+\cdots .
$$

(1) Add a new dependence relation $q_{\gamma_{0} @}=k q_{\delta_{0} @}$. Declare $q_{\gamma_{0} @ \text { is non-active }}$ at stage $s+1$.

(2) We first show that adding $q_{\gamma_{0} @}=k q_{\delta_{0} @}$ does not change the sign of elements in $G_{s}$.

By substituting $q_{\gamma_{0} @}=k q_{\delta_{0}} @ g$ has a new expression

$$
g=\cdots+0 q_{\gamma_{0} @}+\left(r_{\delta_{0}}+r_{\gamma_{0}} k\right) q_{\delta_{0} @}+\cdots .
$$

If $r_{\gamma_{0}}=0$, then the expression of $g$ does not change.

Suppose $r_{\gamma_{0}} \neq 0$. There are two cases:

(i) $r_{\gamma_{0}}>0$. If $r_{\delta_{0}} \geq 0$, then $r_{\delta_{0}}+r_{\gamma_{0}} k>0$. If $r_{\delta_{0}}<0$, since $k>\frac{-r_{\delta_{0}}}{r_{\gamma_{0}}}$, $r_{\delta_{0}}+r_{\gamma_{0}} k>0$

(ii) $r_{\gamma_{0}}<0$. If $r_{\delta_{0}} \leq 0$, then $r_{\delta_{0}}+r_{\gamma_{0}} k<0$. If $r_{\delta_{0}}>0$, since $k>\frac{r_{\delta_{0}}}{-r_{\gamma_{0}}}$, $r_{\delta_{0}}+r_{\gamma_{0}} k<0$.

The current sign of $g$ is calculated as the sign of the first nonzero rational coefficient in this new expression of $g$ if such a nonzero coefficient exists. Hence, the current sign of $g \in G_{s}$ is the same as before. 
(3) We now show that adding $q_{\gamma_{0} @}=k q_{\delta_{0} @}$ ensures that the assignment of $\mathbb{Q}$-linear sums to elements in $G_{s}$ remains one-to-one.

For $x, x^{\prime} \in G_{s}$ with different expression $x=\cdots+a_{\gamma_{0}} q_{\gamma_{0} @}+a_{\delta_{0}} q_{\delta_{0} @}+\cdots$ and $x^{\prime}=\cdots+a_{\gamma_{0}}^{\prime} q_{\gamma_{0} @}+a_{\delta_{0}}^{\prime} q_{\delta_{0} @}+\cdots$. Suppose that the new expression of $x$ and $x^{\prime}$ by substituting $q_{\gamma_{0} @}=k q_{\delta_{0} @}$ is the same.

Then the coefficient of active base-elements at stage $s$ other than $q_{\gamma_{0} @,} q_{\delta_{0} @}$ is the same and

$$
a_{\delta_{0}}+a_{\gamma_{0}} k=a_{\delta_{0}}^{\prime}+a_{\gamma_{0}}^{\prime} k
$$

i.e., $\left(a_{\delta_{0}}-a_{\delta_{0}}^{\prime}\right)=\left(a_{\gamma_{0}}^{\prime}-a_{\gamma_{0}}\right) k$.

(i) If $a_{\delta_{0}}=a_{\delta_{0}}^{\prime}$, then $a_{\gamma_{0}}^{\prime}=a_{\gamma_{0}}$. In this case, the old expression of $x, x^{\prime}$ at stage $s$ is the same, contracting with the assumption on $x, x^{\prime}$.

(ii) If $a_{\delta_{0}} \neq a_{\delta_{0}}^{\prime}$, then $k=\frac{a_{\delta_{0}}-a_{\delta_{0}}^{\prime}}{a_{\gamma_{0}}^{\prime}-a_{\gamma_{0}}}$. However, by the choice of $k, k>$ $\frac{\left|a_{\delta_{0}}-a_{\delta_{0}}^{\prime}\right|}{\left|a_{\gamma_{0}}^{\prime}-a_{\gamma_{0}}\right|}$. So this case is impossible.

Hence, the new expression of $x, x^{\prime} \in G_{s}$ as $\mathbb{Q}$-linear sums of elements in $B_{s}-\left\{q_{\gamma_{0}} @\right.$ is different.

3. If $n>1$, run module $\mathrm{D}(1)$. Otherwise, $n=1$ and $\xi_{1}=\tau$, stop adding dependence relations.

This completes the description of module $\mathrm{D}(0)$.

At the end of module $\mathrm{D}(0)$, each element in $G_{s}$ has a unique $\mathbb{Q}$-linear sum of elements in $B_{s}-\left\{q_{\gamma_{0} @}\right\}$. Now there is no current active base-elements of the form $q_{\zeta} @$ with $\delta_{0} @<_{\text {lex }} \zeta @<_{\text {lex }} \xi_{2} @$.

In general, when $n \geq i+1 \geq 2$, we will start a new module $\mathrm{D}(i)$. Assume that we have already defined $\delta_{i-1} @$ and $\gamma_{i-1} @$, and added dependence relations $q_{\gamma_{j}} @ c_{j} q_{\delta_{j} @}$ for all $j \leq i-1$. Now each element in $G_{s}$ has a unique $\mathbb{Q}$-linear sum of elements in $B_{s}-\left\{q_{\gamma_{j} @}: j \leq i-1\right\}$, and there are no current active base-elements $q_{\zeta @ \text { with }}$ $\delta_{i-1} @<_{\text {lex }} \zeta @<_{\text {lex }} \xi_{i+1} @$. 
Let $\delta_{i} @$ be the length-lexicographically smaller element in $\left\{\delta_{i-1} @, \xi_{i+1} @\right\}$ and $\gamma_{i} @$ be the other element. We will add a dependence relation of the form $q_{\gamma_{i} @}=c_{i} q_{\delta_{i}} @$ in module $\mathrm{D}(i)$.

Module $\mathrm{D}(i)$ with $1 \leq i \leq n-1$.

1. List all nonzero rational numbers which are coefficients of $q_{\delta_{i} @}$ or $q_{\gamma_{i} @}$ in the unique expression of an element in $G_{s}$ as a $\mathbb{Q}$-linear sum of elements in $B_{s}-$ $\left\{q_{\gamma_{j} @: ~} \leq i-1\right\}$ as follows:

$$
r_{1}, r_{2}, \cdots, r_{l}
$$

2. Set $r_{0}:=0$. Choose a large enough $k \in \mathbb{N}^{+}$such that for all $i, i^{\prime}, j, j^{\prime} \leq l$ with $r_{j}-r_{j^{\prime}} \neq 0$

$$
k>\frac{\left|r_{i}-r_{i^{\prime}}\right|}{\left|r_{j}-r_{j^{\prime}}\right|} .
$$

If $\delta_{i} @<_{l e x} \gamma_{i} @$, add a new dependence relation $q_{\gamma_{i} @}=\frac{1}{k} q_{\delta_{i} @}$. If $\gamma_{i} @<_{l e x} \delta_{i} @$, add a new dependence relation $q_{\gamma_{i} @}=k q_{\delta_{i} @}$. Declare $q_{\gamma_{i} @}$ is non-active at stage $s+1$.

By substituting the added dependence relation in the expression of $g \in G_{s}$ as a $\mathbb{Q}$-linear sum of elements in $B_{s}-\left\{q_{\gamma_{j} @}: j \leq i-1\right\}$, we obtain a new expression of $g$ as a $\mathbb{Q}$-linear sum of elements in $B_{s}-\left\{q_{\gamma_{j} @}: j \leq i\right\}$. One can show as in module $\mathrm{D}(0)$ that the sign of $g$ determined by this new expression is unchanged and that the new expression of elements in $G_{s}$ remains one-to-one.

3. When $n=i+1, \xi_{i+1}=\tau$, stop adding dependence relations. Otherwise, $n>i+1$, continue to run module $D(i+1)$.

This completes the description of module $\mathrm{D}(i)$.

Suppose that we have added dependence relations $q_{\gamma_{i} @}=c_{i} q_{\delta_{i} @}$ in module $\mathrm{D}(i)$ for all $i \leq n-1$. By a direct checking, one can show that $q_{\gamma_{i} @}=a_{i} q_{\delta_{n-1} @ \text { for some }}$ $a_{i}>0$. Finally, the set of active base-elements at stage $s+1$ is

$$
B_{s+1}=\left(B_{s}-\left\{q_{\gamma_{i} @}: 0 \leq i \leq n-1\right\}\right) \cup\left\{q_{\sigma} @|\sigma|=s+1\right\} .
$$


This ends adding dependence relations.

We will show in the following sections 4.6 .3 and 4.6.4 that for any set $A, \mathscr{G}$ admits exactly two $A$-computable orders iff $A$ is not PA-complete.

\subsection{Construction and verification}

\subsubsection{Construction}

Fix an effective enumeration $l i n k_{s}$ of $\operatorname{link}$ (a c.e. equivalence relation defined on $\left.2^{<\omega} @\right)$ such that at most one pair is enumerated into $l_{i n k}$ at each stage $s$. Assume that $|\sigma|<s$ and $|\tau|<s$ when $(\sigma @, \tau @) \in \operatorname{link}_{s}$.

At stage $0 . \operatorname{lin}_{0}=\varnothing$. Let $G_{0}=\left\{0_{\mathscr{G}}, q_{@}\right\}$, where $0_{\mathscr{G}}$ is the zero of the constructed group (its unique $\mathbb{Q}$-linear sum is just the empty sum), $q_{@}$ is the active base-element at stage 0 . Set $\operatorname{sign}_{\leq \mathscr{G}}\left(0_{\mathscr{G}}\right)=0$ and $\operatorname{sign}_{\leq \mathscr{G}}\left(q_{@}\right)=+1$.

At stage $s+1$.

Step 1. If a new pair of active base-elements at stage $s$ is enumerated into $\operatorname{link}_{s+1}$, then add dependence relations according to the basic $\mathcal{R}$-strategy, goto step 2. Otherwise, no dependence relations are added at stage $s+1$, goto step 3 .

Step 2. Build $G_{s+1}$ and $\operatorname{sign}_{\leq \mathscr{G}}$ on $G_{s+1}$ respectively according to the basic $\mathcal{P}$-strategy and the basic $\mathcal{Q}$-strategy with dependence relations added.

Step 3. Build $G_{s+1}$ and $s i g n_{\leq \mathscr{g}}$ on $G_{s+1}$ respectively according to the basic $\mathcal{P}$-strategy and the basic $\mathcal{Q}$-strategy with no dependence relations added.

This ends the stage $s+1$ of the construction.

In section 4.6.2, we will show that $\mathcal{P}$ and $\mathcal{Q}$ are both satisfied. In sections 4.6.3 and 4.6.4, we will show that $\mathcal{R}$ is satisfied.

\subsection{2 $\mathscr{G}$ is a computable group possessing computable orders}

Fix an effective enumeration $\mathscr{H}_{s}$ of $\mathscr{H}:=\bigoplus_{\sigma \in 2^{<\omega}} \mathbb{Q} q_{\sigma} @$ such that for all $s, \mid \mathscr{H}_{s+1}-$ $\mathscr{H}_{s} \mid \leq 1$. During the stage $s$ of the construction, we enumerate $q_{\sigma} @$ with $|\sigma|=s$ into $G_{s}$, and obtain active base-elements $B_{s}$. We may enumerate one more element, 
say $x$, into $G_{s}$; in this case, there is an element $y$ in $\mathscr{H}_{s}$ such that $x$ is a unique $\mathbb{Q}$-linear sum of elements in $B_{s}$ obtained by replacing non-active base-elements in $y$ by active ones with corresponding scaling, and no elements enumerated into $G$ so far has an expression the same as $x$.

For each $g \in G_{s}$, it can be expressed as a unique $\mathbb{Q}$-linear combination of active base-elements at stage $s$. Now we show that the expressions of $g$ only change finitely many times after stage $s$. Then each element in $G$ has a unique $\mathbb{Q}$-linear combination of active base-elements at end.

We only need to show that each original base-element, say $q_{\sigma @}$, has finitely many expressions (all such expressions are elements of $\mathscr{H}$, they represent the same element of $\mathscr{G}) . q_{\sigma} @$ is first enumerated into $G$ as an active base-element at stage $|\sigma|$, it has a new expression later only if there is a $\tau @<_{\text {len-lex }} \sigma @ \operatorname{such}$ that $\operatorname{link}(\sigma @, \tau @)$ holds (in this case, $q_{\sigma @}$ will be non-active forever). Then $q_{\sigma @}=a q_{\tau @}$ for some $a>0$.

Since $\leq_{\text {len-lex }}$ is a well ordering on $2^{<\omega} @$, there is a length-lexicographically least element, say $\rho @$, in $2^{<\omega} @$ such that $\operatorname{link}(\sigma @, \rho @)$ holds. Then $q_{\sigma} @$ has a last expression of the form $b q_{\rho @}$ for some $b>0$. In this case, $q_{\rho @}$ is active forever.

For each $g \in G_{s}$, we also define the sign $\operatorname{sign}_{\leq \mathscr{G}}(g)$ such that if $g$ has a new expression later, then the sign computed according to this new expression is the same as before. Now for each element, $g$ say, in $\mathscr{G}$, it may have several expressions in terms of elements in $\mathscr{H}$; however, $\operatorname{sign}_{\leq \mathscr{G}}(g)$ is just the sign computed according to the first expression of $g$.

$\mathscr{G}$ is computable. For two original elements, say $g, h$, in $\mathscr{H}$, one can effectively check whether they represent the same element in $\mathscr{G}$ (i.e. $g$ equals $h$ in $\mathscr{G}$ ) as follows: (1) find the stage $t$ such that $g-h$ is first enumerated into $\mathscr{H}$; (2) at stage $t$, we will consider $g-h$ and update it as a $\mathbb{Q}$-linear sum, say $x$, of elements in $B_{t} ;(3)$ $g-h=0_{\mathscr{G}}$ iff all coefficients in $x$ are 0 . Hence, this equality relation on $\mathscr{H}$ is computable. $\mathscr{G}$ can be viewed as the quotient group of $\mathscr{H}$ modulo this equality relation. Thus, $\mathscr{G}$ is computable.

$\leq_{\mathscr{G}}$ is computable. Now $\operatorname{sign}_{\leq \mathscr{G}}(g)$ is a computable function on $\mathscr{G}$. Moreover, $P:=\left\{g \in \mathscr{G}: \operatorname{sign}_{\leq \mathscr{G}}(g)=+1\right\} \cup\{0 \mathscr{G}\}$ is a computable set which is closed under addition, containing exactly one of $g$ and $-g$ when $g \neq 0 \mathscr{G}$. That is, $P$ is a positive cone on $\mathscr{G}$. So $P$ determines a computable order, namely $\leq \mathscr{G}$, on $\mathscr{G}$. 
$\mathscr{G}$ is $\Delta_{2}^{0}$-isomorphic to $\mathbb{Q}^{\infty}$. Since there are infinitely many infinite paths on $T$, the so defined link-relation on $2^{<\omega} @$ has infinitely many equivalence classes, that is, $B:=\left\{q_{\sigma @}: \sigma \in 2^{<\omega}, q_{\sigma @}\right.$ is active $\}$ is infinite. Moreover, $B$ forms a $\Pi_{1}^{0}$-basis for $\mathscr{G}$, and $\mathscr{G}$ is isomorphic to $\underset{b \in B}{\oplus} \mathbb{Q} b$ via a $\Delta_{2}^{0}$-isomorphism. Hence, $\mathscr{G}$ is $\Delta_{2}^{0}$-isomorphic to $\mathbb{Q}^{\infty}$.

\subsubsection{A-computable orders on $\mathscr{G}$ which are not computable}

Suppose that $\leq^{A}$ is an $A$-computable order on $\mathscr{G}$ such that $\leq^{A} \neq \leq \mathscr{G}$ and $\leq^{A} \neq \leq_{\mathscr{G}}^{*}$. We will show that $A$ computes an infinite path on the co-c.e. binary tree $T$ which is PA-complete. Then $A$ is also PA-complete.

Note that $\mathscr{G}$ is just $\mathscr{H}$ modulo the so defined computable equality relation, denoted by $\sim$. For any order on $\mathscr{G}$ with corresponding sign function, say $\varphi$, we can indeed extend $\varphi$ to $\mathscr{H}$ by setting $\varphi(h)=\varphi(g)$ where $g \in \mathscr{G}$ such that $g \sim h$. In particular, we will talk about the sign of elements in $\mathscr{H}$ under $\leq^{A}$ or $\leq_{\mathscr{G}}$ through this way.

We start by comparing $\leq^{A}$ with $\leq_{\mathscr{G}}$ on $\left\{q_{\sigma @}: \sigma \in 2^{<\omega}\right\}$, the set of original base-elements. The sign of each $q_{\sigma @}$ under $\leq_{\mathscr{G}}$ is +1 . For the sign determined by $\leq{ }^{A}$ or simply called $A$-sign, either there are two base-elements, say $q_{\sigma @}$ and $q_{\tau @}$, having different $A$-sign; or all base-elements have the same $A$-sign.

Case 1. Suppose that there are $q_{\sigma} @$ and $q_{\tau} @$ such that their $A$-sign are different. Since each base-element is nonzero in $\mathscr{G}$, its $A$-sign is not 0 . Suppose that the $A$-sign of $q_{\sigma} @$ is -1 , and that the $A$-sign of $q_{\tau} @$ is +1 .

Claim. link $(\sigma @, \tau @)$ fails.

Proof. Suppose otherwise. We will add a dependence relation of the form $q_{\sigma @}=$ $r q_{\tau} @$ with $r>0, \tau @<_{\text {len-lex }} \sigma @$ or of the form $q_{\tau @}=r^{\prime} q_{\sigma @}$ with $r^{\prime}>0, \sigma @<_{\text {len-lex }}$ $\tau @$. In both cases, $q_{\tau @}$ and $q_{\sigma} @$ have the same $A$-sign.

This ends the proof.

Recall that $\sigma @$ and $\tau @$ are not linked means that there is at least one infinite path on $T$ such that it is lexicographically between $\sigma @$ and $\tau @$.

With the help of $\leq^{A}$, we will initiate an $A$-effective process which approximates lexicographically form below and above an infinite path of $T$. Without loss of generality, assume that $\tau @<_{\text {lex }} \sigma @$. 
Let $\xi_{0} @=\tau @, \zeta_{0} @=\sigma @$. By assumption, $q_{\xi_{0} @}$ and $q_{\zeta_{0} @ \text { have } A \text {-sign }+1 \text { and }}$ -1 respectively. Let $\delta_{0}$ be the longest common initial segment of $\xi_{0}, \zeta_{0}$, denoted by $\xi_{0} \cap \zeta_{0}$. Then any infinite binary string lexicographically between $\xi_{0} @$ and $\zeta_{0} @$ extends $\delta_{0}$. Start module $\mathrm{C}(0)$ below, where one can determine a strictly extension $\delta_{1}$ of $\delta_{0}$ such that $\delta_{1}$ is a prefix of the desired infinite path (we are searching for) on $T$.

In general module $\mathrm{C}(i)$ with $i \geq 0$, we define $\delta_{i+1} \supset \delta_{i}$ such that $\delta_{i+1}$ can be extended into an infinite path on $T$. $\mathrm{C}(i)$ proceeds as follows.

Module $\mathrm{C}(i)$ with $i \geq 0$.

1. Pick the length-lexicographically least element $\eta \in 2^{<\omega}$ with $\xi_{i} @<_{l e x} \eta @<_{\text {lex }}$ $\zeta_{i} @$, denoted by $\eta_{i}$.

2. If $q_{\eta_{i}} @$ has $A$-sign -1, then update $\xi_{i+1} @=\xi_{i} @, \zeta_{i+1} @=\eta_{i} @$.

If $q_{\eta_{i}} @$ has $A$-sign +1 , then update $\xi_{i+1} @=\eta_{i} @, \zeta_{i+1} @=\zeta_{i} @$.

In both cases, $q_{\xi_{i+1} @}$ and $q_{\zeta_{i+1} @}$ have different $A$-sign. So there is an infinite path on $T$ which is lexicographically between $\xi_{i+1} @$ and $\zeta_{i+1} @$.

3. Now define $\delta_{i+1} \supset \delta_{i}$ such that there is an infinite binary string on $T$ extending $\delta_{i+1}$. There are three cases.

3.1. If $\xi_{i} @<_{\text {lex }} \delta_{i} @<_{\text {lex }} \zeta_{i} @$, then $\delta_{i}$ is the length-lexicographically least element $\eta$ in $2^{<\omega}$ such that $\xi_{i} @<_{\text {lex }} \eta @<_{\text {lex }} \zeta_{i} @$, i.e., $\eta_{i}=\delta_{i}$.

(i) If $\zeta_{i+1}=\eta_{i}$, then any infinite binary string lexicographically between $\xi_{i+1} @$ and $\zeta_{i+1} @$ extends $\delta_{i} 0$. Just set $\delta_{i+1}=\delta_{i} 0$.

(ii) If $\xi_{i+1}=\eta_{i}$, then any infinite binary string lexicographically between $\xi_{i+1} @$ and $\zeta_{i+1} @$ extends $\delta_{i} 1$. Just set $\delta_{i+1}=\delta_{i} 1$.

3.2. If $\delta_{i} @ \leq_{l e x} \xi_{i} @$, then $\delta_{i} 1 \subseteq \eta_{i}$. In this case, any infinite binary string lexicographically between $\xi_{i+1} @$ and $\zeta_{i+1} @$ extends $\delta_{i} 1$. So set $\delta_{i+1}=\delta_{i} 1$. 
3.3. If $\zeta_{i} @ \leq_{\text {lex }} \delta_{i} @$, then $\delta_{i} 0 \subseteq \eta_{i}$. In this case, any infinite binary string lexicographically between $\xi_{i+1} @$ and $\zeta_{i+1} @$ extends $\delta_{i} 0$. So set $\delta_{i+1}=\delta_{i} 0$.

As $\operatorname{link}\left(\xi_{i+1} @, \zeta_{i+1} @\right)$ fails, there is an infinite path, say $f$, on $T$ which is lexicographically between $\xi_{i+1} @$ and $\zeta_{i+1} @$. Since any infinite binary string lexicographically between $\xi_{i+1} @$ and $\zeta_{i+1} @$ extends $\delta_{i+1}$, $f$ extends $\delta_{i+1}$.

4. Start module $\mathrm{C}(i+1)$.

This completes module $\mathrm{C}(i)$.

We obtain a strictly increasing sequence $\left\{\delta_{i}: i \in \mathbb{N}\right\}$ of binary strings such that each $\delta_{i}$ can be extended into an infinite path on $T$. Let $g=\cup_{i \in \mathbb{N}} \delta_{i}$, i.e., $g(n)=\delta_{n+1}(n)$ (note that $\left|\delta_{n+1}\right| \geq n+1$ ). Then $g$ is the desired infinite path on $T$ which is computable from $\leq^{A}$, thus $g$ is $A$-computable. Since $g$ is PA-complete, $A$ is also PA-complete.

Case 2. Suppose that each original base-element has the same $A$-sign under $\leq^{A}$. Suppose that this $A$-sign is +1 , we will show that $\leq^{A}$ can compute an infinite path of $T$ [otherwise, we can deal with the reversal order of $\leq^{A}$, and then show that this reversal order can compute an infinite path of $T$ ].

Now for each $q_{\sigma @}$, its $A$-sign is the same as $\operatorname{sign}_{\leq \mathscr{G}}\left(q_{\sigma}\right)$. Since $\leq^{A} \neq \leq \mathscr{G}$, there is an element, say $g \in G$, such that $\operatorname{sign}_{\leq \mathscr{G}}(g)=+1$, but the $A$-sign of $g$ is -1 . $g$ has a final $\mathbb{Q}$-linear sum of active base-elements, denoted by $g=a_{0} q_{\sigma_{0} @}+\cdots+a_{n} q_{\sigma_{n}} @$ with $a_{i} \neq 0$ for each $i=0, \cdots, n$ and $\sigma_{0} @<_{l e x} \sigma_{1} @ \cdots<_{l e x} \sigma_{n} @$.

As $\operatorname{sign}_{\leq \mathscr{G}}(g)=+1, a_{0}>0$. We may assume $a_{0}=1$ [otherwise we deal with $\frac{1}{a_{0}} g$ ]. Now write $g$ as

$$
g=\left[q_{\sigma_{0} @}+\left(a_{1}-1\right) q_{\sigma_{1} @}\right]+\cdots+\left[q_{\sigma_{m-1} @}+\left(a_{m}-1\right) q_{\sigma_{m} @}\right]+\cdots+q_{\sigma_{n} @} .
$$

For each $1 \leq m \leq n, \operatorname{sign}_{\leq \mathscr{G}}\left(q_{\sigma_{m-1} @}+\left(a_{m}-1\right) q_{\sigma_{m} @}\right)=+1$, and $\operatorname{sign}_{\leq \mathscr{G}}\left(q_{\sigma_{n} @}\right)=+1$. Note that $q_{\sigma_{n}} @$ has $A$-sign +1 . Since the $A$-sign of $g$ is -1 , there is some $m \leq n$ such that $q_{\sigma_{m-1}} @+\left(a_{m}-1\right) q_{\sigma_{m}} @$ has $A$-sign -1. In this case, $a_{m}-1<0$; otherwise, $a_{m}-1 \geq 0$, then $q_{\sigma_{m-1}} @+\left(a_{m}-1\right) q_{\sigma_{m}} @$ has $A$-sign +1 , a contradiction. 
Hence, there are $\sigma @, \tau @$ with $\sigma @<_{\text {lex }} \tau @$ and $r>0$ such that $q_{\sigma @}-r q_{\tau} @$ has sign +1 under $\leq_{\mathscr{G}}$ and -1 under $\leq{ }^{A}$ respectively.

Claim. For such $\sigma @, \tau @, \operatorname{link}(\sigma @, \tau @)$ fails.

Proof. Suppose otherwise. There are two cases:

If $\tau @<_{\text {len-lex }} \sigma @$, then a dependence relation of the form $q_{\sigma @}=a q_{\tau} @$ with $a>0$ will be added such that $0 q_{\sigma} @+(a-r) q_{\tau} @$ has sign +1 under $\leq \mathscr{G}$. So $a-r>0$.

In this case, since the $A$-sign of $q_{\tau} @$ is +1 , the $A$-sign of $0 q_{\sigma @}+(a-r) q_{\tau @}$ is +1 . As $q_{\sigma @}-r q_{\tau @}=0 q_{\sigma @}+(a-r) q_{\tau @ \text { in }} \mathscr{G}$, if $\leq^{A}$ is an order on $\mathscr{G}$, by our convention, the $A$-sign of $q_{\sigma @}-r q_{\tau} @$ and $0 q_{\sigma} @(a-r) q_{\tau} @$ is the same, contradicting the assumption that $q_{\sigma @}-r q_{\tau} @$ has $A$-sign -1 .

If $\sigma @<_{\text {len-lex }} \tau @$, then we will add a dependence relation of the form $q_{\tau @}=b q_{\sigma @}$ with $b>0$ such that $\operatorname{sign}_{\leq \mathscr{G}}\left((1-r b) q_{\sigma @}+0 q_{\tau} @\right)=+1$. So $1-r b>0$. Then $(1-r b) q_{\sigma @}+0 q_{\tau} @$ has $A$-sign +1 , a contradiction.

This ends the proof.

Similar to case 1 , we will initiate an $A$-effective process which approximates an infinite path on $T$ lexicographically form below and above.

Find $(\sigma @, \tau @)$ with $\sigma @<_{l e x} \tau @$ and $r>0$ such that $q_{\sigma @}-r q_{\tau} @$ has sign +1 under $\leq_{\mathscr{G}}$ and sign -1 under $\leq^{A}$. Let $\xi_{0} @=\sigma @, \zeta_{0} @=\tau @$ and $r_{0}=r$. Note that any infinite binary string lexicographically between $\xi_{0} @$ and $\zeta_{0} @$ extends $\delta_{0}:=\xi_{0} \cap \zeta_{0}$.

First start module $\widehat{\mathrm{C}}(0)$, where we will determine the successor, say $j$, of $\delta_{0}$ such that there is an infinite path on $T$ which extends $\delta_{0} j$.

In general, for $i \geq 1$, suppose that we have already obtained $\xi_{i} @<_{\text {lex }} \zeta_{i} @$, $r_{i}>0$, and $\delta_{i}$ in module $\widehat{\mathrm{C}}(i-1)$, where $\operatorname{sign}_{\leq_{\mathscr{G}}}\left(q_{\xi_{i} @}-r_{i} q_{\zeta_{i} @}\right)=+1$, the $A$-sign of $q_{\xi_{i} @}-r_{i} q_{\zeta_{i} @}$ is -1 , and there is an infinite binary string on $T$ which extends $\delta_{i}$. Then we will start module $\widehat{\mathrm{C}}(i)$ to search for longer approximation $\delta_{i+1}$ of an infinite path on $T$. We point out here that for $i \geq 1, \delta_{i}$ may not be $\xi_{i} \cap \zeta_{i}$, but the point is that any infinite binary string lexicographically between $\xi_{i} @$ and $\zeta_{i} @$ extends $\delta_{i}$.

Module $\widehat{\mathrm{C}}(i)$ with $i \geq 0$.

1. Pick the length-lexicographically least element $\eta \in 2^{<\omega}$ such that $\xi_{i} @<_{\text {lex }}$ $\eta @<_{\text {lex }} \zeta_{i} @$, denoted by $\eta_{i}$. 
2. Search until a positive $r^{\prime}$ is found such that $q_{\xi_{i} @}-r^{\prime} q_{\eta_{i} @}$ and $q_{\eta_{i} @}-\frac{r_{i}}{r^{\prime}} q_{\zeta_{i} @}$ have the same sign +1 under $\leq \mathscr{G}$; and either $q_{\xi_{i} @}-r^{\prime} q_{\eta_{i} @}$ or $q_{\eta_{i} @}-\frac{r_{i}}{r^{\prime}} q_{\zeta_{i} @ \text { has sign }}$ -1 under $\leq^{A}$.

We now show that such a $r^{\prime}$ exists. Indeed, since $\operatorname{link}\left(\xi_{i} @, \zeta_{i} @\right)$ fails, there is an infinite binary string, say $f$, on $T$ which is lexicographically between $\xi_{i} @$ and $\zeta_{i} @$. There are three cases:

2.1. If $\xi_{i} @<_{\text {lex }} f<_{\text {lex }} \eta_{i} @\left[\operatorname{so} \operatorname{link}\left(\xi_{i} @, \eta_{i} @\right)\right.$ fails], and $\operatorname{link}\left(\eta_{i} @, \zeta_{i} @\right)$ holds.

(i) $\eta_{i} @<_{\text {len-lex }} \zeta_{i} @$. Then $q_{\zeta_{i} @}=a q_{\eta_{i} @ \text { for some } a>0 \text {, and }}$

$$
q_{\eta_{i} @}-\frac{r_{i}}{r^{\prime}} q_{\zeta_{i} @}=\left(1-\frac{r_{i} a}{r^{\prime}}\right) q_{\eta_{i} @}+0 q_{\zeta_{i} @} .
$$

One can choose a positive $r^{\prime}$ such that $1-\frac{r_{i} a}{r^{\prime}}>0$.

(ii) $\zeta_{i} @<_{\text {len-lex }} \eta_{i} @$. Then $q_{\eta_{i} @}=b q_{\zeta_{i} @ \text { for some } b>0 \text {, and }}$

$$
q_{\eta_{i} @}-\frac{r_{i}}{r^{\prime}} q_{\zeta_{i} @}=0 q_{\eta_{i}} @+\left(b-\frac{r_{i}}{r^{\prime}}\right) q_{\zeta_{i} @} .
$$

One can choose a positive $r^{\prime}$ such that $b-\frac{r_{i}}{r^{\prime}}>0$.

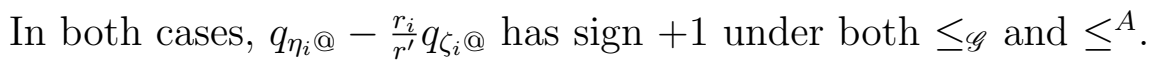

Since $\operatorname{link}\left(\xi_{i} @, \eta_{i} @\right)$ fails (no relation will be added between $q_{\xi_{i} @}$ and

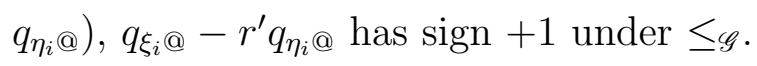

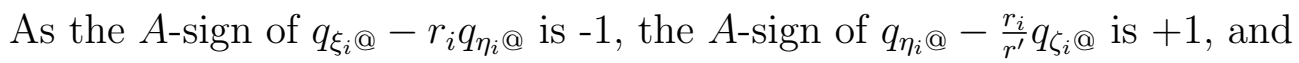

$$
q_{\xi_{i} @}-r_{i} q_{\eta_{i} @}=\left(q_{\xi_{i} @}-r^{\prime} q_{\eta_{i} @}\right)+r^{\prime}\left(q_{\eta_{i} @}-\frac{r_{i}}{r^{\prime}} q_{\zeta_{i} @}\right),
$$

the $A$-sign of $q_{\xi_{i} @}-r^{\prime} q_{\eta_{i} @}$ is -1 .

2.2. If $\eta_{i} @<_{\text {lex }} f<_{\text {lex }} \zeta_{i} @\left[\operatorname{so} \operatorname{link}\left(\eta_{i} @, \zeta_{i} @\right)\right.$ fails], and $\operatorname{link}\left(\xi_{i} @, \eta_{i} @\right)$ holds.

(i) $\xi_{i} @<_{\text {len-lex }} \eta_{i} @$. Then $q_{\eta_{i} @}=a q_{\xi_{i}} @$ for some $a>0$, and

$$
q_{\xi_{i} @}-r^{\prime} q_{\eta_{i} @}=\left(1-r^{\prime} a\right) q_{\xi_{i} @}+0 q_{\eta_{i} @} .
$$

One can choose a positive $r^{\prime}$ such that $1-r^{\prime} a>0$. 
(ii) $\eta_{i} @<_{\text {len-lex }} \xi_{i} @$. Then $q_{\xi_{i} @}=b q_{\eta_{i} @ \text { for some } b>0 \text {, and }}$

$$
q_{\xi_{i} @}-r^{\prime} q_{\eta_{i} @}=0 q_{\xi_{i} @}+\left(b-r^{\prime}\right) q_{\eta_{i} @} .
$$

One can choose a positive $r^{\prime}$ such that $b-r^{\prime}>0$.

Similarly, one can show that $q_{\xi_{i} @}-r^{\prime} q_{\eta_{i} @ \text { and }} q_{\eta_{i} @}-\frac{r_{i}}{r^{\prime}} q_{\zeta_{i} @ \text { have sign }+1}$ under $\leq \mathscr{G}$; and that $q_{\xi_{i} @}-r^{\prime} q_{\eta_{i} @ \text { and }} q_{\eta_{i} @}-\frac{r_{i}}{r^{\prime}} q_{\zeta_{i} @}$ have $A$-sign +1 and -1 respectively.

2.3. If $\operatorname{link}\left(\xi_{i} @, \eta_{i} @\right)$ and $\operatorname{link}\left(\eta_{i} @, \zeta_{i} @\right)$ both fail. Clearly, $q_{\xi_{i} @}-q_{\eta_{i} @}$ and $q_{\eta_{i} @}-r_{i} q_{\zeta_{i} @}$ have sign +1 under $\leq \mathscr{G}$. Since the $A$-sign of $q_{\xi_{i} @}-r_{i} q_{\zeta_{i} @}$ is -1 , and

$$
q_{\xi_{i} @}-r_{i} q_{\zeta_{i} @}=\left(q_{\xi_{i} @}-q_{\eta_{i} @}\right)+\left(q_{\eta_{i} @}-r_{i} q_{\zeta_{i} @}\right),
$$

at least one of $q_{\xi_{i} @}-q_{\eta_{i} @}$ and $q_{\eta_{i} @}-r_{i} q_{\zeta_{i} @}$ has $A$-sign -1. In this case, just pick $r^{\prime}=1$.

3. If the $A$-sign of $q_{\xi_{i} @}-r^{\prime} q_{\eta_{i} @}$ is -1, update $\xi_{i+1} @=\xi_{i} @, \zeta_{i+1} @=\eta_{i} @, r_{i+1}=r^{\prime}$. If the $A$-sign of $q_{\eta_{i}} @-\frac{r_{i}}{r^{\prime}} q_{\zeta_{i}}$ is -1 , update $\xi_{i+1} @=\eta_{i} @, \zeta_{i+1} @=\zeta_{i} @, r_{i+1}=\frac{r_{i}}{r^{\prime}}$. In both cases, $\operatorname{sign}_{\leq \mathscr{G}}\left(q_{\xi_{i+1} @}-r_{i+1} q_{\zeta_{i+1} @}\right)=+1$, but the A-sign of $q_{\xi_{i+1} @}-$ $r_{i+1} q_{\zeta_{i+1} @}$ is -1. Hence, $\operatorname{link}\left(\xi_{i+1} @, \zeta_{i+1} @\right)$ fails. There is an infinite path on $T$ which is lexicographically between $\xi_{i+1} @$ and $\zeta_{i+1} @$.

4. Define $\delta_{i+1} \supset \delta_{i}$ such that there is an infinite path on $T$ extending $\delta_{i+1}$ as in the step 3 of module $\mathrm{C}(i)$ in case 1 .

5. Start module $\widehat{\mathrm{C}}(i+1)$.

This completes module $\widehat{\mathrm{C}}(i)$.

After running all modules $\widehat{\mathrm{C}}(i)$ with $i \geq 0$, we arrive at an increasing sequence $\left\{\delta_{i}: i \in \mathbb{N}\right\}$ such that there is an infinite path on $T$ extending each $\delta_{i}$. Let $g=\cup_{i \in \mathbb{N}} \delta_{i}$. Then $g$ is an $A$-computable path on $T$, thus, $A$ is PA-complete.

In sum, we have showed that every order on $\mathscr{G}$ is either computable or computable form a PA-complete set. Hence, if $A$ is not PA-complete, then every $A$-computable on $\mathscr{G}$ is computable. 


\subsubsection{Orders on $\mathscr{G}$ which are computable from PA-complete sets}

Recall that the set of infinite paths of the co-c.e. tree $T$ is just the set of $\{0,1\}$ valued diagonally nonrecursive functions. Let $A$ be any PA-complete set. Then $A$ computes an infinite path on $T$. In order to prove that there is an $A$-computable order on $\mathscr{G}$ which is not computable, we only need to show that any infinite path on $T$ computes a noncomputable order on $\mathscr{G}$.

Let $f \in[T]$. Now build a $f$-sign on $G$ which determines a $f$-computable order, $\leq^{f}$ say, on $\mathscr{G}$. For each original base-element $q_{\sigma @}$,

(1) if $\sigma @<_{\text {lex }} f$, then set the $f$-sign of $q_{\sigma} @$ to be +1 ;

(2) if $f<_{\text {lex }} \sigma @$, then set the $f$-sign of $q_{\sigma @}$ to be -1 .

We then build the $f$-sign of $\leq^{f}$ by a similar algorithm as in building the sign of $\leq \mathscr{G}$.

For instance, assume that $x \in G_{s}$ has a unique $\mathbb{Q}$-linear sum of active baseelements at stage $s$ as follows:

$$
x=a_{0} q_{\sigma_{0} @}+\cdots+a_{i} q_{\sigma_{i} @}+b_{0} q_{\tau_{0} @}+\cdots+b_{j} q_{\tau_{j} @},
$$

where $b_{j} \neq 0, \sigma_{0} @<_{\text {lex }} \cdots<_{\text {lex }} \sigma_{i} @<_{\text {lex }} f<_{\text {lex }} \tau_{0} @<_{\text {lex }} \cdots<_{\text {lex }} \tau_{j} @$.

(1) If there is a $k \leq i$ such that $a_{k} \neq 0$, then find such a least $k$, and set the $f$-sign of $x$ to be the sign of $a_{k}$.

(2) Otherwise, let $k^{\prime} \leq j$ be the least number with $b_{k^{\prime}} \neq 0$, and set the $f$-sign of $x$ to be the sign of $-b_{k^{\prime}}$.

Furthermore, if the expression of $x$ changes later because some base-elements become non-active, then the algorithm preserves the $f$-sign of $x$. This ensures that this sign function is well-defined on $\mathscr{G}$.

Clearly, the sign function is $f$-computable, so the corresponding order $\leq^{f}$ is $f$ computable. Since there are $\sigma @, \tau @ \in 2^{<\omega} @$ such that $\sigma @<_{l e x} f<_{l e x} \tau @, q_{\sigma} @$ and $q_{\tau} @$ have different sign under $\leq^{f}$. Then $\leq^{f}$ is neither $\leq_{\mathscr{G}}$ nor the reversal of $\leq_{\mathscr{G}}$, that is, $\leq^{f}$ is not computable.

We have constructed a computable group $\mathscr{G}$ such that for any set $A, \mathscr{G}$ admits exactly two $A$-computable orders iff $A$ is not PA-complete.

This completes the proof of Theorem 4.1. 


\section{Chapter 5}

\section{Group orders and c.e. degrees}

In this chapter, we will show that for any nonzero c.e. degree $\mathbf{a}$, there is a nonzero c.e. degree $\mathbf{c}<\mathbf{a}$ and a computable group $\mathscr{G}$ such that $\mathscr{G}$ has orders of degree $\geq \mathbf{a}$ and $\mathbf{c}$ is group-order-computable via $\mathscr{G}$, which implies that $\mathscr{G}$ has no incomputable orders of degree $\leq \mathbf{c}$.

Theorem 5.1 For every noncomputable c.e. set A, there is a noncomputable c.e. set $C \leq_{T} A$ and a computable torsion-free abelian group $\mathscr{G}$ with infinite rank such that

(1) $\mathscr{G}$ has exactly two computable orders;

(2) $\mathscr{G}$ has an A-computable basis;

(3) every $C$-computable order on $\mathscr{G}$ is computable.

For a computable torsion-free abelian group $\mathscr{A}$ with infinite rank, if $\mathscr{A}$ has a basis $B$, then the set $\mathcal{O}(\mathscr{A})$ of degrees of orders on $\mathscr{A}$ contains $\{\mathbf{d}: \mathbf{d} \geq \operatorname{deg}(B)\}$. Hence, our method is to control the degree of the constructed basis $B:=\left\{b_{i}: i \in \mathbb{N}\right\}$ of $\mathscr{G}$. If $\operatorname{deg}(B) \leq \mathbf{a}$, then the constructed $\mathscr{G}$ also satisfies

$$
\{\mathbf{d}: \mathbf{d} \geq \mathbf{a}\} \subseteq\{\mathbf{d}: \mathbf{d} \geq \operatorname{deg}(B)\} \subseteq \mathcal{O}(\mathscr{G}) .
$$

\subsection{Requirements and strategies}

Let $\left\{\mathscr{H}_{i}: i \in \mathbb{N}\right\}$ be a uniformly computable sequence of subgroups with rank one, and let $\mathscr{H}=\bigoplus_{i \in \mathbb{N}} \mathscr{H}_{i}$ be the direct sum of $\mathscr{H}_{i}(i \in \mathbb{N})$. As in [3], we will build a computable group $\mathscr{G}$ isomorphic to $\mathscr{H}$ which admits computable orders. 
To prove Theorem 5.1, we will build a computable group $\mathscr{G}$, a computable group order $\leq_{\mathscr{G}}$ on $\mathscr{G}$, and a c.e. set $C \leq_{T} A$ to satisfy the following requirements.

$\mathcal{P}_{e}: W_{e} \neq \bar{C}$.

$\mathcal{Q}: \mathscr{G}$ has an $A$-computable basis $B=\left\{b_{i}: i \in \mathbb{N}\right\}$

$\mathcal{Q}_{0}: \mathscr{G} \cong \mathscr{H}$

$\mathcal{Q}_{1}: \leq_{\mathscr{G}}$ and $\leq_{\mathscr{G}}^{*}$ are exactly two computable orders on $\mathscr{G}$.

$\mathcal{N}_{e}:$ If $\leq_{e}^{C}$ is a $C$-computable group order on $\mathscr{G}$, then either $\leq_{e}^{C}=\leq_{\mathscr{G}}$ or $\leq_{e}^{C}=\leq_{\mathscr{G}}^{*}$.

Where $\left\{W_{e}: e \in \mathbb{N}\right\}$ is an enumeration of c.e. subsets of natural numbers, and $\left\{\Phi_{e}: e \in \mathbb{N}\right\}^{1}$ is an enumeration of partial computable functionals defined on $\mathscr{G} \times \mathscr{G}$, $\bar{C}$ is the complement of $C$. The symbol $\leq_{e}^{C}$ stands for the $C$-computable binary relation on $\mathscr{G}$ computed by $\Phi_{e}^{C}$; specifically, we view for any $x, y \in \mathscr{G}, x \leq_{e}^{C} y$ iff $\Phi_{e}^{C}(x, y) \downarrow=1$. If $\Phi_{e}^{C}(x, y) \downarrow$, then let $\phi_{e}^{C}(x, y)$ denote the use for the computation $\Phi_{e}^{C}(x, y)$.

To ensure $C \leq_{T} A$, we will add an $A$-permitting to the standard $\mathcal{P}_{e}$-strategy. The $\mathcal{Q}$-strategy ensures that $\mathscr{G}$ has an $A$-computable basis. The $\mathcal{Q}_{0}$-strategy and the $\mathcal{Q}_{1}$-strategy are similar to corresponding strategies in Theorem 1.17. We will develop new $\mathcal{N}$-strategies.

\subsubsection{A $\mathcal{P}_{e}$-strategy with permitting}

$\mathcal{P}_{e}$ is a standard Friedberg-Muchnik strategy with permitting. So we use cycles, we start with cycle 0 and cycle $k$ with $k \geq 0$ proceeds as follows (assume $y_{-1}=0$ ).

(1) Choose a big witness $y_{k}>y_{k-1}$.

(2) Wait for $y_{k}$ enumerating into $W_{e}$ at some stage, say $s_{k}$.

(3) Open cycle $k+1$ and simultaneously, wait for $A_{s_{k}} \Upsilon_{y_{k}} \neq A_{s} \Upsilon_{y_{k}}$ at some stage $s \geq s_{k}$.

\footnotetext{
${ }^{1}$ Here, we explain $\Phi_{e}$ in more detail. We first fix an effective numbering, say $\iota$, of $\mathscr{G}$ (i.e., $\iota$ is a computably bijective mapping from $\mathbb{N}$ to $\mathscr{G})$. Let $\left\{\Theta_{e}: e \in \mathbb{N}\right\}$ be an enumeration of partial computable functionals defined on $\mathbb{N} \times \mathbb{N}$. Then for any $X \subseteq \mathbb{N}$, and $x, y \in \mathscr{G}, \Phi_{e}^{X}(x, y) \simeq$ $\Theta_{e}^{X}\left(\iota^{-1}(x), \iota^{-1}(y)\right)$.
} 
(4) Put $y_{k}$ into $C_{s}$, and close all cycles.

Suppose that the $\mathcal{P}_{e^{-}}$strategy opens infinitely many cycles. Then each cycle $k$ is waiting at (3) forever, that is, we find witnesses $y_{k} \in W_{e}$ and stages $s_{k}$ such that $A_{s_{k}} \Upsilon_{y_{k}}=A \uparrow_{y_{k}}$. Then we can compute $A$ as follows: let $x \in \mathbb{N}$, to decide whether it is in $A$ or not, find a least cycle $k_{x}$ such that $y_{k_{x}}>x$. Then $x \in A \Leftrightarrow x \in A_{s_{k_{x}}}$.

Hence, by the noncomputability of $A$, there are only finitely many cycles opened during the construction. If there is a first cycle $k$ reaching at (4), then all cycles are closed and $y_{k} \in C \cap W_{e}$. Otherwise, no cycles are closed, and there is a least cycle $k$ waiting at (2) forever; in this case, cycle $k$ is the largest cycle opened, $y_{k} \notin W_{e}$ and also $y_{k} \notin C$. In both cases, $\mathcal{P}_{e}$ is satisfied.

We can compute $C$ by $A$ as follows: let $x \in \mathbb{N}$, find a least stage $s_{x}$ such that $A_{s_{x}} \uparrow_{x}=A \uparrow_{x}(=\{y \in A: y \leq x\})$, then $x \in C \Leftrightarrow x \in C_{s_{x}}$.

\subsubsection{The $\mathcal{Q}_{0}$-strategy}

The $\mathcal{Q}_{0}$-strategy is a global strategy developed in [3]. To build the group $\mathscr{G}$ isomorphic to $\mathscr{H}$, we maintain an approximation $\left\{b_{0}^{s}, \cdots, b_{s}^{s}\right\}$ of a basis $\left\{b_{i}=\lim _{s} b_{i}^{s}: i \in \mathbb{N}\right\}$ of $\mathscr{G}=\bigoplus_{i \in \mathbb{N}} \mathscr{G}_{i}$ and an approximation $\left\{N_{0}^{s}, \cdots, N_{s}^{s}\right\}$ of a basis $\left\{N_{i}=\lim _{s} N_{i}^{s}: i \in \mathbb{N}\right\}$ of $\mathscr{H}=\bigoplus_{i \in \mathbb{N}} \mathscr{H}_{i}$ at each stage $s$ such that

(1) $\left\{b_{i}\right\}$ and $\left\{N_{i}\right\}$ is respectively a basis of rank one groups $\mathscr{G}_{i}$ and $\mathscr{H}_{i}$;

(2) the isomorphism $\Omega: \mathscr{G}=\left(G, 0_{\mathscr{G}},+\mathscr{G}\right) \rightarrow \mathscr{H}$ of abelian groups is determined by sending each $b_{i}$ to $N_{i}$.

As each computable torsion-free abelian group of rank one can be effectively embedded into $\mathbb{Q}$, we view the subgroup $\mathscr{H}_{i}$ of $\mathscr{H}$ as a subgroup of additive group of rational numbers. So $N_{i}^{s} \in \mathscr{H}_{i} \subseteq \mathbb{Q}$ and $N_{i} \in \mathbb{Q}$.

\section{Construction of $\mathscr{G}$.}

At stage 0. $G_{0}=\left\{0_{\mathscr{G}}, b_{0}^{0}\right\}$, where $0_{\mathscr{G}}$ is the zero element of $\mathscr{G}$ and $\left\{b_{0}^{0}\right\}$ is the approximation basis corresponding to $N_{0}^{0}=1$.

At the end of stage $s, G_{s}$ is the set containing all elements of $\mathscr{G}$ enumerated by stage $s$, and each nonzero $g \in G_{s}$ has assigned a unique $\mathbb{Q}$-linear sum $q_{0}^{s} b_{0}^{s}+\cdots+q_{n}^{s} b_{n}^{s}$, 
where $q_{n}^{s} \neq 0(n \leq s)$, each $q_{i}^{s}(i \leq n)$ in $\mathbb{Q}$ satisfying $q_{i}^{s} N_{i}^{s} \in \mathscr{H}_{i}$ at stage $s$. By convention, $0_{\mathscr{G}}$ is assigned the empty sum.

At stage $s+1$.

Case 1. If no dependence relations are added among approximation basis ele-

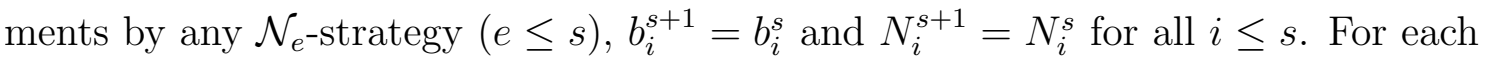
$g \in G_{s}$, it has the same expression as before at stage $s+1$. That is, if $q_{0}^{s} b_{0}^{s}+\cdots+q_{n}^{s} b_{n}^{s}$ is the $\mathbb{Q}$-linear sum of approximation basis elements at stage $s$, then let $q_{i}^{s+1}=q_{i}^{s}$ for all $i \leq n$ and $g$ has the $\mathbb{Q}$-linear sum $q_{0}^{s+1} b_{0}^{s+1}+\cdots+q_{n}^{s+1} b_{n}^{s+1}$ at stage $s+1$.

Let $N_{s+1}^{s+1}=1$ and add a new element $b_{s+1}^{s+1}$ into approximation basis. Find the first tuple $<x_{0}, \cdots, x_{m}>$ of rationals (under a fixed effective numbering of the set of all finite sequences of rationals) with $x_{m} \neq 0$ and $m \leq s$ such that for all $i \leq m$, $x_{i} N_{i}^{s+1} \in \mathscr{H}_{i}$ at stage $s+1$, and the $\mathbb{Q}$-linear sum $x_{0} b_{0}^{s+1}+\cdots+x_{m} b_{m}^{s+1}$ is not assigned to elements of the constructed group. If such a tuple exist, then enumerate $x_{0} b_{0}^{s+1}+\cdots+x_{m} b_{m}^{s+1}$ into $G_{s+1}$.

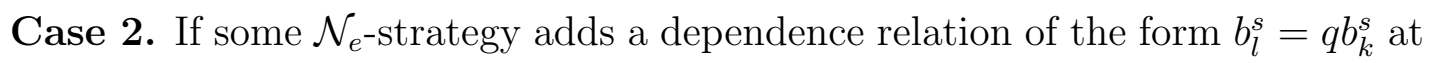
stage $s$ with $l$ an odd number, $k$ an even number $(e<k<l), q$ a rational number.

To ensure the uniqueness of $\mathbb{Q}$-linear sums at each stage, $q$ also satisfies additional conditions. We require that for any two elements in $G_{s}$, if their $\mathbb{Q}$-linear sums of approximation basis elements at stage $s$ are different, then after substituting the relation $b_{l}^{s}=q b_{k}^{s}$, their new $\mathbb{Q}$-linear sums are still different.

We only need to consider $\mathbb{Q}$-linear sums involving basis elements $b_{k}^{s}$ and $b_{l}^{s}$.

- For example, assume that $g=x b_{k}^{s}+y b_{l}^{s}, h=x^{\prime} b_{k}^{s}+y^{\prime} b_{l}^{s}$, and either $x \neq x^{\prime}$ or $y \neq y^{\prime}$. If now after substituting $b_{l}^{s}=q b_{k}^{s},(x+y q) b_{k}^{s}=\left(x^{\prime}+y^{\prime} q\right) b_{k}^{s}$, i.e., $x+y q=x^{\prime}+y^{\prime} q$. Then $y \neq y^{\prime}$ and $q=\frac{x^{\prime}-x}{y-y^{\prime}}$.

Thus, for any two different elements $f, g$ in $G_{s}$, if after substituting the relation $b_{l}^{s}=q b_{k}^{s}$, the new expression of $f, g$ at stage $s+1$ is the same, then one can compute a unique $q(f, g)$ such that $q=q(f, g)$. As $G_{s}$ is a finite set, there are finitely many such $q(f, g)$. We will choose the desired $q$ different from all $q(f, g)$ with $f \neq g$ in $G_{s}$ but $f, g$ has the same expression at stage $s+1$ when adding $b_{l}^{s}=q(f, g) b_{k}^{s}$. Then for any two different elements in $G_{s}$, their assigned sums remain different at stage $s+1$. 
Now we update basis approximation elements. Let $b_{i}^{s+1}=b_{i}^{s}$ for all $i \leq s$ with $i \neq l$, and $N_{i}^{s+1}=N_{i}^{s}$ for all $i \leq s$ with $i \neq k$. Add two new elements $b_{l}^{s+1}$ and $b_{s+1}^{s+1}$ into approximation basis. Set $N_{s+1}^{s+1}=1$. We now define $N_{k}^{s+1}$.

Let $g \in G_{s}$.

- Assume that $g=q_{0}^{s} b_{0}^{s}+\cdots+q_{k}^{s} b_{k}^{s}+\cdots+q_{l}^{s} b_{l}^{s}+\cdots+q_{n}^{s} b_{n}^{s}$ at stage $s$. Now $g$ changes to

$$
q_{0}^{s} b_{0}^{s}+\cdots+\left(q_{k}^{s}+q_{l}^{s} q\right) b_{k}^{s}+\cdots+0 b_{l}^{s}+\cdots+q_{n}^{s} b_{n}^{s} .
$$

Let $q_{i}^{s+1}=q_{i}^{s}$ for all $i \leq n$ with $i \notin\{k, l\}, q_{k}^{s+1}=q_{k}^{s}+q_{l}^{s} q, q_{l}^{s+1}=0$. Then at stage $s+1, g=q_{0}^{s+1} b_{0}^{s+1}+\cdots+q_{n}^{s+1} b_{n}^{s+1}$.

To ensure the $\mathbb{Q}$-linear sum of $g$ is well-defined after substituting the dependence relation $b_{l}^{s}=q b_{k}^{s}$, we need $q_{i}^{s+1} N_{i}^{s+1} \in \mathscr{H}_{i}$ for all $i \leq n$. For $i \notin\{k, l\}, q_{i}^{s+1} N_{i}^{s+1}=$ $q_{i}^{s} N_{i}^{s} \in \mathscr{H}_{i}$; note that $q_{l}^{s+1} N_{l}^{s+1}=0 \in \mathscr{H}_{l}$ is already true. So we only require $q_{k}^{s+1} N_{k}^{s+1} \in \mathscr{H}_{k}$.

Now define $N_{k}^{s+1}=d_{q} d N_{k}^{s}$, where $d_{q}$ is the denominator of $q$ and $d$ is the product of all $d_{q_{l}^{s}}$ such that $d_{q_{l}^{s}}$ is the denominator of nonzero $q_{l}^{s}$, and $q_{l}^{s} b_{l}^{s}$ appears in the $\mathbb{Q}$-linear sum of an element in $G_{s}$. Then $q d_{q} \in \mathbb{Z}$ and $q_{l}^{s} d \in \mathbb{Z}$, so

$$
q_{k}^{s+1} N_{k}^{s+1}=\left(q_{k}^{s}+q_{l}^{s} q\right) N_{k}^{s+1}=d_{q} d q_{k}^{s} N_{k}^{s}+q_{l}^{s} d q d_{q} N_{k}^{s} \in \mathscr{H}_{k} .
$$

Finally, search the first tuple $<x_{0}, \cdots, x_{m}>$ and add the $\mathbb{Q}$-linear sum $x_{0} b_{0}^{s+1}+$ $\cdots+x_{m} b_{m}^{s+1}$ into $G_{s+1}$ as in case 1 .

Case 3. If some $\mathcal{N}_{e}$-strategy adds a dependence relation of the form $b_{l}^{s}=$ $m_{1} b_{j}^{s}-m_{2} b_{k}^{s}$ at stage $s$ with $l$ an odd number, $l>j, k$ an even number $(e<k<l)$, $m_{1}, m_{2}$ integers.

The first condition of $m_{1}, m_{2}$ is to keep the assignment of sums of elements in $G_{s}$ remaining one-to-one after substituting the relation $b_{l}^{s}=m_{1} b_{j}^{s}-m_{2} b_{k}^{s}$.

- For example, assume that $g=x b_{j}^{s}+y b_{k}^{s}+z b_{l}^{s}, h=x^{\prime} b_{j}^{s}+y^{\prime} b_{k}^{s}+z^{\prime} b_{l}^{s}$, and $g \neq h$ in $G_{s}$, that is, $x \neq x^{\prime}$ or $y \neq y^{\prime}$ or $z \neq z^{\prime}$. If

$$
\left(x+z m_{1}\right) b_{j}^{s}+\left(y-z m_{2}\right) b_{k}^{s}=\left(x^{\prime}+z^{\prime} m_{1}\right) b_{j}^{s}+\left(y^{\prime}-z^{\prime} m_{2}\right) b_{k}^{s},
$$

then $x+z m_{1}=x^{\prime}+z^{\prime} m_{1}$ and $y-z m_{2}=y^{\prime}-z^{\prime} m_{2}$. So $z \neq z^{\prime}, m_{1}=\frac{x^{\prime}-x}{z-z^{\prime}}$, and $m_{2}=\frac{y-y^{\prime}}{z-z^{\prime}}$. 
In general, for $f \neq g$ in $G_{s}$, so their expressions as $\mathbb{Q}$-linear sum of approximation basis elements are different at stage $s$. If $f, g$ has the same expression at stage $s+1$ after substituting the relation $b_{l}^{s}=m_{1} b_{j}^{s}-m_{2} b_{k}^{s}$, then for $i=1,2$, we can compute a unique $m_{i}(f, g)$ such that $m_{i}=m_{i}(f, g)$. Note that there are finitely many such $m_{i}(f, g)$, the desired $m_{i}$ will be different from all those $m_{i}(f, g)$ with $f \neq g$ in $G_{s}$. Then the $\mathbb{Q}$-linear sum of elements in $G_{s}$ remains one-to-one at stage $s+1$.

Let $b_{i}^{s+1}=b_{i}^{s}$ for all $i \leq s$ with $i \neq l$ and $N_{i}^{s+1}=N_{i}^{s}$ for all $i \leq s$. Add two new elements $b_{l}^{s+1}$ and $b_{s+1}^{s+1}$ into approximation basis, and set $N_{s+1}^{s+1}=1$.

The second conditions on $m_{1}, m_{2}$ are to ensure that the $\mathbb{Q}$-linear sums are welldefined after substituting the dependence relation $b_{l}^{s}=m_{1} b_{j}^{s}-m_{2} b_{k}^{s}$. That is, for any $g \in G_{s}$,

- assume that $g=q_{0}^{s} b_{0}^{s}+\cdots+q_{j}^{s} b_{j}^{s}+\cdots+q_{k}^{s} b_{k}^{s}+\cdots+q_{l}^{s} b_{l}^{s}+\cdots+q_{n}^{s} b_{n}^{s}$. Now rewrite $g$ as

$$
q_{0}^{s} b_{0}^{s}+\cdots+\left(q_{j}^{s}+q_{l}^{s} m_{1}\right) b_{j}^{s}+\cdots+\left(q_{k}^{s}-q_{l}^{s} m_{2}\right) b_{k}^{s}+\cdots+0 b_{l}^{s}+\cdots+q_{n}^{s} b_{n}^{s} .
$$

Let $q_{i}^{s+1}=q_{i}^{s}$ for all $i \leq n$ with $i \notin\{j, k, l\}, q_{j}^{s+1}=q_{j}^{s}+q_{l}^{s} m_{1}, q_{k}^{s+1}=q_{k}^{s}-q_{l}^{s} m_{2}$, $q_{l}^{s+1}=0$. Then

$$
g=q_{0}^{s+1} b_{0}^{s+1}+\cdots+q_{n}^{s+1} b_{n}^{s+1} .
$$

We need to ensure $q_{i}^{s+1} N_{i}^{s+1} \in \mathscr{H}_{i}$ for all $i \leq n$.

Note that $q_{i}^{s+1} N_{i}^{s+1} \in \mathscr{H}_{i}$ for $i \notin\{k, j, l\}$ and $q_{l}^{s+1} N_{l}^{s+1}=0 \in \mathscr{H}_{l}$. We only require $q_{j}^{s+1} N_{j}^{s+1} \in \mathscr{H}_{j}$ and $q_{k}^{s+1} N_{k}^{s+1} \in \mathscr{H}_{k}$.

$$
q_{j}^{s+1} N_{j}^{s+1}=\left(q_{j}^{s}+q_{l}^{s} m_{1}\right) N_{j}^{s+1}=q_{j}^{s} N_{j}^{s}+q_{l}^{s} m_{1} N_{j}^{s} .
$$

Choose $m_{1}$ to be the multiple of the product $d$ of all $d_{q_{l}^{s}}$ such that $d_{q_{l}^{s}}$ is the denominator of $q_{l}^{s}$, where $q_{l}^{s} \neq 0$ and $q_{l}^{s} b_{l}^{s}$ appears in the $\mathbb{Q}$-linear sum of an element in $G_{s}$. Then $q_{l}^{s} m_{1} \in \mathbb{Z}$ and $q_{j}^{s+1} N_{j}^{s+1} \in \mathscr{H}_{j}$. Similarly, choose $m_{2}$ with $d \mid m_{2}$, we have

$$
q_{k}^{s+1} N_{k}^{s+1}=\left(q_{k}^{s}-q_{l}^{s} m_{2}\right) N_{k}^{s+1}=q_{k}^{s} N_{k}^{s}-q_{l}^{s} m_{2} N_{k}^{s} \in \mathscr{H}_{k} .
$$

At last, find the first tuple $\left\langle x_{0}, \cdots, x_{m}>\right.$ of rational numbers and add the $\mathbb{Q}$-linear sum $x_{0} b_{0}^{s+1}+\cdots+x_{m} b_{m}^{s+1}$ into $G_{s+1}$ as in case 1 . 
For $f, g, h \in G_{s}$, define $f+{ }_{s} g=h$ if the addition of $\mathbb{Q}$-linear sums of $f$ and $g$ is equal to the $\mathbb{Q}$-linear sum of $h$. By linearity of additions, when updating expressions of $f, g, h$ at stage $s+1$, we still have $f+_{s+1} g=h$.

This completes the construction of $\mathscr{G}$ at stage $s+1$.

Let $\Omega_{s}: G_{s} \rightarrow \mathscr{H}$ be the linear extension of mapping which sends each $b_{i}^{s}$ to $N_{i}^{s}$. Then $\Omega=\lim _{s} \Omega_{s}$ is a $\Delta_{2}^{0}$-isomorphism from $\mathscr{G}$ to $\mathscr{H}$ such that $\Omega\left(b_{i}\right)=N_{i}$ for all $i$.

\subsubsection{The $\mathcal{Q}_{1}$-strategy}

The $\mathcal{Q}_{1}$-strategy is also a global strategy developed in [3]. Similar to the $\mathcal{Q}_{0}$-strategy, at each stage $s$, we maintain approximation elements $\left\{r_{0}^{s}, \cdots, r_{s}^{s}\right\}$ in $\mathbb{R}$ which is linearly independent over $\mathbb{Q}$, and map $b_{i}^{s}$ to $r_{i}^{s}$. We choose each $r_{i}^{s}(1 \leq i \leq s)$ as a positive rational multiple of $\sqrt{p_{i}}$, where $p_{i}$ is the $i$-th prime number for $i \geq 1$.

For $i \geq 1, r_{i}=\lim _{s} r_{i}^{s} . r_{0}=r_{0}^{s}=1_{\mathbb{R}}$ for all $s$.

(1) Let $\Theta$ be the linear extension of the map $\Psi: \mathscr{G} \rightarrow\left(\mathbb{R}, 0_{\mathbb{R}},+_{\mathbb{R}}\right) ; b_{i} \mapsto r_{i}$;

(2) Let $\Theta(\mathscr{G})$ be the image of $\mathscr{G}$ under $\Theta$, then $\mathscr{G}$ is isomorphic to the subgroup $\Theta(\mathscr{G})$ of $\left(\mathbb{R}, 0_{\mathbb{R}},+_{\mathbb{R}}\right)$ and $\left\{r_{i}: i \in \mathbb{N}\right\}$ is a basis of $\Theta(\mathscr{G})$.

The linear order $\leq_{\mathscr{G}}$ will satisfy $x \leq_{\mathscr{G}} y \Leftrightarrow \Theta(x) \leq_{\mathbb{R}} \Theta(y)$ for any $x, y \in \mathscr{G}$, this definition does not ensure the computability of $\leq_{\mathscr{G}}$. To make $\leq_{\mathscr{G}}$ computable, we will approximate $\leq_{\mathscr{G}}$ via $\leq_{s}$ at each stage $s$, and keep $x \leq_{s} y$ implies $x \leq_{t} y$ for all stages $t \geq s$.

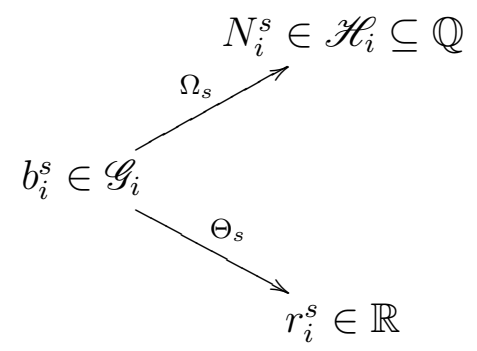

At each stage $s$, each approximation basis $b_{i}^{s}(i \leq s)$ will be mapped to $r_{i}^{s}$ in $\mathbb{R}$, and we have an approximation $\Theta_{s}$ of $\Theta$ which is a linear extension of mapping on approximation basis elements. $\Theta=\lim _{s} \Theta_{s}$ is a $\Delta_{2}^{0}$ map from $\mathscr{G}$ to $\mathbb{R}$. Although at 
limit level, the linear order $\leq_{\mathscr{G}}$ is defined by $\Theta$ which is a linear extension on basis elements of $\mathscr{G}$, we cannot simply define the approximation $\leq_{s}$ of $\leq_{\mathscr{G}}$ at stage $s$ via $\Theta_{s}$ as: $g \leq_{s} h \Leftrightarrow \Theta_{s}(g) \leq_{\mathbb{R}} \Theta_{s}(h)$.

The obstacle is when some $\mathcal{N}_{e}$-strategy adding dependence relations of the form $b_{l}^{s}=q b_{k}^{s}$ or $b_{l}^{s}=m_{1} b_{j}^{s}-m_{2} b_{k}^{s}$ at stage $s+1$, if $\leq_{s+1}$ is really defined by $\Theta_{s+1}$, note $\Theta_{s}\left(b_{l}^{s}\right)=r_{l}^{s}$, this means that $b_{l}^{s}$ maps to new positions $\Theta_{s+1}\left(b_{l}^{s}\right)=q r_{k}^{s}$ or $\Theta_{s+1}\left(b_{l}^{s}\right)=$ $m_{1} r_{j}^{s}-m_{2} r_{k}^{s}$ in $\mathbb{R}$ at stage $s+1$ (we will see $r_{k}^{s+1}=r_{k}^{s}$ and $r_{j}^{s+1}=r_{j}^{s}$ ). We may change the relative positions of two elements in $G_{s}$, that is, maybe $\Theta_{s}(x)<_{\mathbb{R}} \Theta_{s}(y)$ but $\Theta_{s+1}(y)<_{\mathbb{R}} \Theta_{s+1}(x)$. So we cannot ensure that $\leq_{\mathscr{G}}$ is computable.

The method used in [3] is to assign rational-endpoint interval $\left(a_{i}^{s}, \widehat{a}_{i}^{s}\right)$ to each basis element $b_{i}^{s}$ to control the position $r_{i}^{s}$ of $b_{i}^{s}$, namely, let $r_{i}^{s} \in\left(a_{i}^{s}, \widehat{a}_{i}^{s}\right)$ and $\widehat{a}_{i}^{s}-a_{i}^{s}<\mathbb{R} \frac{1}{2^{s}}$, and then control the position of each $g \in G_{s}$ in $\mathbb{R}$ by assigning a rational-endpoint interval $\left(a_{g}^{s}, \widehat{a}_{g}^{s}\right)$ for $g$ based on the intervals of basis elements. That is, if $g=$ $x_{0} b_{0}^{s}+\cdots+x_{n} b_{n}^{s}$, then

(i) $a_{g}^{s}=x_{0} \sigma\left(a_{0}^{s}\right)+\cdots+x_{n} \sigma\left(a_{n}^{s}\right)$ with $\sigma\left(a_{i}^{s}\right)=a_{i}^{s}$ if $x_{i} \geq_{\mathbb{R}} 0, \sigma\left(a_{i}^{s}\right)=\widehat{a}_{i}^{s}$ if $x_{i}<_{\mathbb{R}} 0$;

(ii) $\widehat{a}_{g}^{s}=x_{0} \tau\left(\widehat{a}_{0}^{s}\right)+\cdots+x_{n} \tau\left(\widehat{a}_{n}^{s}\right)$ with $\tau\left(\widehat{a}_{i}^{s}\right)=\widehat{a}_{i}^{s}$ if $x_{i} \geq_{\mathbb{R}} 0, \tau\left(\widehat{a}_{i}^{s}\right)=a_{i}^{s}$ if $x_{i}<_{\mathbb{R}} 0$.

Now define $\leq_{s}$ via assigned intervals as follows.

(1) Let $g \leq_{s} g$ for each $g \in G_{s}$.

(2) For each $g, h \in G_{s}$, if $\left(a_{g}^{s}, \widehat{a}_{g}^{s}\right) \cap\left(a_{h}^{s}, \widehat{a}_{h}^{s}\right)=\varnothing$ and $\widehat{a}_{g}^{s}<_{\mathbb{R}} a_{h}^{s}$, then $g<_{s} h$.

In order to make $\leq_{\mathscr{G}}$ computable, we choose approximation intervals such that

$$
r_{i}^{s+1} \in\left(a_{i}^{s+1}, \widehat{a}_{i}^{s+1}\right) \subseteq\left(a_{i}^{s}, \widehat{a}_{i}^{s}\right)
$$

and $\widehat{a}_{i}^{s+1}-a_{i}^{s+1}<_{\mathbb{R}} \frac{1}{2^{s+1}}$. Then for each $g \in G_{s},\left(a_{g}^{s+1}, \widehat{a}_{g}^{s+1}\right) \subset\left(a_{g}^{s}, \widehat{a}_{g}^{s}\right)$. Hence, for all $g, h \in G_{s}, g<_{s} h$ implies $g<_{s+1} h$.

Note that $b_{k}^{s}$ never changes for even $k$, as in [3], we also require that $r_{k}^{s}$ close to $0_{\mathbb{R}}$ and choose $\widehat{a}_{k}^{s}<\mathbb{R} \frac{1}{2^{s}}$ for positive even $k$. This helps us to deal with witnesses for $\mathcal{N}$-strategies.

Construction of $\leq \mathscr{G}$. 
At stage 0. $G_{0}=\left\{0_{\mathscr{G}}, b_{0}^{0}\right\}, 0_{\mathscr{G}}$ sends to $0_{\mathbb{R}}$, and set $0_{\mathscr{G}}<_{0} b_{0}^{0}$ by defining $r_{0}=1_{\mathbb{R}}$. Assume that we have defined $\left\{r_{0}^{s}, \cdots, r_{s}^{s}\right\}$ by stage $s$.

At stage $s+1$.

Case 1. If no dependence relations are added via any $\mathcal{N}_{e^{-}}$strategy. For each $i \leq s, r_{i}^{s+1}=r_{i}^{s}$. Choose rational numbers $a_{i}^{s+1}, \widehat{a}_{i}^{s+1}$ such that $r_{i}^{s+1} \in\left(a_{i}^{s+1}, \widehat{a}_{i}^{s+1}\right) \subseteq$ $\left(a_{i}^{s}, \widehat{a}_{i}^{s}\right)$ and $\widehat{a}_{i}^{s+1}-a_{i}^{s+1}<_{\mathbb{R}} \frac{1}{2^{s+1}}$. If $i$ is even, we also let $\widehat{a}_{i}^{s+1}<_{\mathbb{R}} \frac{1}{2^{s+1}}$.

Add a new element of the form $r_{s+1}^{s+1}=q_{s+1} \sqrt{p_{s+1}}$ with $q_{s+1}$ a positive rational number. Choose $a_{s+1}^{s+1}, \widehat{a}_{s+1}^{s+1}$ such that $r_{s+1}^{s+1} \in\left(a_{s+1}^{s+1}, \widehat{a}_{s+1}^{s+1}\right)$ and $\widehat{a}_{s+1}^{s+1}-a_{s+1}^{s+1}<_{\mathbb{R}} \frac{1}{2^{s+1}}$. If $s+1$ is even, we also require $\widehat{a}_{s+1}^{s+1}<_{\mathbb{R}} \frac{1}{2^{s+1}}$.

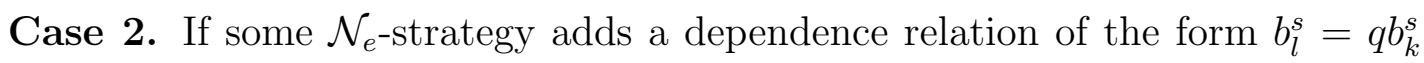
with $q \in \mathbb{Q}^{+}$or $b_{l}^{s}=m_{1} b_{j}^{s}-m_{2} b_{k}^{s}$ with $m_{1}, m_{2} \in \mathbb{Z}$. For each $i \leq s$ with $i \neq l$, set $r_{i}^{s+1}=r_{i}^{s}$ and $\operatorname{assign}\left(a_{i}^{s+1}, \widehat{a}_{i}^{s+1}\right)$ as in case 1 . For $b_{l}^{s}$,

(1) if $b_{l}^{s}=q b_{k}^{s}$, choose $q$ such that the new interval $\left(q a_{k}^{s+1}, q \widehat{a}_{k}^{s+1}\right)$ for $b_{l}^{s}$ at stage $s+1$ is contained in $\left(a_{l}^{s}, \widehat{a}_{l}^{s}\right)$;

(2) if $b_{l}^{s}=m_{1} b_{j}^{s}-m_{2} b_{k}^{s}$ with $m_{1}, m_{2} \in \mathbb{Z}^{+}$, choose $m_{1}, m_{2}$ such that the new interval $\left(m_{1} a_{j}^{s+1}-m_{2} \widehat{a}_{k}^{s+1}, m_{1} \widehat{a}_{j}^{s+1}-m_{2} a_{k}^{s+1}\right)$ for $b_{l}^{s}$ at stage $s+1$ is contained in $\left(a_{l}^{s}, \widehat{a}_{l}^{s}\right)$;

(3) if $b_{l}^{s}=m_{1} b_{k}^{s}-m_{2} b_{j}^{s}$ with $m_{1}, m_{2} \in \mathbb{Z}^{+}$, choose $m_{1}, m_{2}$ such that the new interval $\left(m_{1} a_{k}^{s+1}-m_{2} \widehat{a}_{j}^{s+1}, m_{1} \widehat{a}_{k}^{s+1}-m_{2} a_{j}^{s+1}\right)$ for $b_{l}^{s}$ at stage $s+1$ is contained in $\left(a_{l}^{s}, \widehat{a}_{l}^{s}\right)$.

Let $r_{l}^{s+1}=\sqrt{p_{l}}$, corresponding to new $b_{l}^{s+1}$. Choose new rational numbers $a_{l}^{s+1}, \widehat{a}_{l}^{s+1}$ such that $r_{l}^{s+1} \in\left(a_{l}^{s+1}, \widehat{a}_{l}^{s+1}\right)$ and $\widehat{a}_{l}^{s+1}-a_{l}^{s+1}<_{\mathbb{R}} \frac{1}{2^{s+1}}$. Choose a new $r_{s+1}^{s+1}$ for $b_{s+1}^{s+1}$ and assign interval $\left(a_{s+1}^{s+1}, \widehat{a}_{s+1}^{s+1}\right)$ as in case 1 .

This completes the construction of $\leq_{\mathscr{G}}$ at stage $s+1$.

\subsubsection{The $\mathcal{Q}$-strategy}

In order to satisfy the $\mathcal{Q}$-requirement, we modify the $\mathcal{N}_{e}$-strategy developed in [3] through the following way: we add dependence relations of the form $b_{l}^{s}=q b_{k}^{s}$ or 
$b_{l}^{s}=m b_{k}^{s}+n b_{j}^{s}$ for some odd $l$ at stage $w$ only when permitted by $A$, that is, $A_{w-1} \Upsilon_{l} \neq A_{w} \Upsilon_{l}$. Then for any stage $t, A_{t} \Upsilon_{l}=A \Upsilon_{l}$ implies $b_{l}=b_{l}^{t}$.

The main idea is that for same active witness of $\mathcal{N}_{e}$, we may search for multiple diagonalization witnesses until permitted by $A$, and then add dependence relations [here, active witness and diagonalization witness will be defined in the basic $\mathcal{N}_{e^{-}}$ strategy]. As $A$ is assumed to be incomputable, such a permitting occurs; otherwise, we can initiate an effective process for computing $A$.

Hence, $B=\left\{b_{i}=\lim _{s} b_{i}^{s}: i \in \mathbb{N}\right\} \leq_{T} A$. Indeed, for an arbitrary $g \in \mathscr{G}$, let $s_{g}$ be the first stage when $g$ enumerates into $\mathscr{G}$. If $g$ is neither $b_{s_{g}}^{s_{g}}$ nor $b_{l}^{s_{g}}$ with odd $l<s_{g}$, then $g \notin B$. Otherwise, $g=b_{s_{g}}^{s_{g}}$ or $g=b_{l}^{s_{g}}$ for some odd $l$ (in this case, we add dependence relations on $b_{l}^{s_{g}-1}$, and $b_{l}^{s_{g}}$ is the new approximation for $b_{l}$ ),

(1) if $g=b_{s_{g}}^{s_{g}}$ with $s_{g}$ even or $g=b_{l}^{s_{g}}$ for some odd $l<s_{g}$, then $g \in B$;

(2) if $g=b_{s_{g}}^{s_{g}}$ with $s_{g}$ odd, find a least stage $t \geq s_{g}$ such that $A_{t} \uparrow_{s_{g}}=A \uparrow_{s_{g}}$, then $g \in B$ iff $g=b_{s_{g}}^{t}=b_{s_{g}}$.

$\mathcal{N}_{e}$ still acts finitely often, so the whole construction is still a finite injury argument.

\subsubsection{A $\mathcal{N}_{e}$-strategy}

We need to ensure that if $\leq_{e}^{C} \neq \leq_{\mathscr{G}}$ and $\leq_{e}^{C} \neq \leq_{\mathscr{G}}^{*}$, then $\leq_{e}^{C}$ is not a group order on $\mathscr{G}$. A basic $\mathcal{N}_{e}$-strategy works as follows.

(A1) Wait for an active witness $\left(b_{j}^{s}, n, b_{k}^{s}\right)$ at some stage $s$, namely, $\left(b_{j}^{s}, n, b_{k}^{s}\right)$ satisfying (a1), (a2), (a3) or (a1), (a2), (a4).

(a1) $k$ is an even number larger than $e$.

(a2) $0 \mathscr{G}_{G}<_{s} n b_{k}^{s}<_{s} b_{j}^{s}<_{s}(n+1) b_{k}^{s}$.

(a3) $b_{k}^{s}>_{e}^{C} 0_{\mathscr{G}}$ and either $b_{j}^{s}<_{e}^{C} n b_{k}^{s}$ or $b_{j}^{s}>_{e}^{C}(n+1) b_{k}^{s}$.

(a4) $b_{k}^{s}>_{e}^{C^{*}} 0_{\mathscr{G}}$ and either $b_{j}^{s}<_{e}^{C^{*}} n b_{k}^{s}$ or $b_{j}^{s}>_{e}^{C^{*}}(n+1) b_{k}^{s}$.

By Lemma 3.9 [3], as long as $\leq_{e}^{C}$ is a group order on $\mathscr{G}$ satisfying $\leq_{e}^{C} \neq \leq_{\mathscr{G}}$ and $\leq_{e}^{C} \neq \leq_{\mathscr{G}}^{*}$, we can find active witnesses for $\leq_{e}^{C}$. That is, if no active witnesses appeared during the construction, then either $\leq_{e}^{C}$ is not a group order on $\mathscr{G}$ or $\leq_{e}^{C}=\leq_{\mathscr{G}}$ or $\leq_{e}^{C}=\leq_{\mathscr{G}}^{*}$, and hence $\mathcal{N}_{e}$ is satisfied. 
(A2) We first set a restraint $r_{2}^{C}\left(b_{j}^{s}, n, b_{k}^{s}\right)$ on $C$ to preserve the computations orderings in (a3) or (a4) for $\leq_{e}^{C}$ at stage $s$. Say that $\mathcal{N}_{e}$ has active witnesses $\left(b_{j}^{s}, n, b_{k}^{s}\right)$ now.

For the active witness $\left(b_{j}^{s}, n, b_{k}^{s}\right)$, assume that we are in case (a3), we deal with (a4) similarly by considering the reveral order $\leq_{e}^{C^{*}}$.

As long as $\left(b_{j}^{s}, n, b_{k}^{s}\right)$ is not initialized, that is, the $C$-restraint $r_{2}^{C}\left(b_{j}^{s}, n, b_{k}^{s}\right)$ still exist and $b_{j}^{s}$ is not canceled, we use the mechanism of cycles to assign multiple diagonalization witnesses for $\left(b_{j}^{s}, n, b_{k}^{s}\right)$. Cycles can be opened or closed, and cycle $i$ can open cycle $i+1$. Assume that we have opened cycles $n<i$. During cycle $i$ with $i \geq 0$, proceed as follows:

(D1) At the least odd stage $t_{i}>t_{i-1} \geq s$ ( set $t_{-1}=s$ ), declare that the new approximate basis element $b_{t_{i}}^{t_{i}}$ is a diagonalization witness for $\left(b_{j}^{s}, n, b_{k}^{s}\right)$ at cycle $i$. Order $b_{t_{i}}^{t_{i}}$ as in (b1) or (b2) when (b0) holds.

(b0) For all stages $v$ with $s \leq v \leq t_{i}, b_{j}^{v}=b_{j}^{s}, C_{s} \uparrow_{r_{2}^{C}\left(b_{j}^{s}, n, b_{k}^{s}\right)}=C_{t_{i}} \uparrow_{r_{2}^{C}\left(b_{j}^{s}, n, b_{k}^{s}\right)}$.

(b1) If we have $b_{j}^{s}<_{e}^{C} n b_{k}^{s}$ in (a3), set $n b_{k}^{s}<_{t_{i}} b_{t_{i}}^{t_{i}}<_{t_{i}} b_{j}^{s}$, that is,

- define $r_{t_{i}}^{t_{i}}=q_{t_{i}} \sqrt{p_{t_{i}}}$ for some positive rational $q_{t_{i}}$ and choose $a_{t_{i}}^{t_{i}}, \widehat{a}_{t_{i}}^{t_{i}}$ such that $n \widehat{a}_{k}^{t_{i}}<_{\mathbb{R}} a_{t_{i}}^{t_{i}}<_{\mathbb{R}} r_{t_{i}}^{t_{i}}<_{\mathbb{R}} \widehat{a}_{t_{i}}^{t_{i}}<_{\mathbb{R}} a_{j}^{t_{i}}$.

(b2) If we have $b_{j}^{s}>_{e}^{C}(n+1) b_{k}^{s}$ in (a3), set $b_{j}^{s}<_{t_{i}} b_{t_{i}}^{t_{i}}<_{t_{i}}(n+1) b_{k}^{s}$, that is,

- define $r_{t_{i}}^{t_{i}}=q_{t_{i}} \sqrt{p_{t_{i}}}$ for some positive rational $q_{t_{i}}$ and choose $a_{t_{i}}^{t_{i}}$, $\widehat{a}_{t_{i}}^{t_{i}}$ such that $\widehat{a}_{j}^{t_{i}}<_{\mathbb{R}} a_{t_{i}}^{t_{i}}<_{\mathbb{R}} r_{t_{i}}^{t_{i}}<_{\mathbb{R}} \widehat{a}_{t_{i}}^{t_{i}}<_{\mathbb{R}}(n+1) a_{k}^{t_{i}}$.

We call $\left(b_{t_{i}}^{t_{i}}, b_{j}^{s}, n, b_{k}^{s}\right)$ a pre-potentially permanent witness at cycle $i$ of the active witnesses $\left(b_{j}^{s}, n, b_{k}^{s}\right)$ of $\mathcal{N}_{e}$. Proceed as follows to add dependence relations.

(D2) Wait for a potentially permanent witness $\left(b_{t_{i}}^{u}, b_{j}^{s}, n, b_{k}^{s}\right)$ at some stage $u>t_{i}$, namely, $\left(b_{t_{i}}^{u}, b_{j}^{s}, n, b_{k}^{s}\right)$ satisfying $(\mathrm{c} 1),(\mathrm{c} 2)$ or $(\mathrm{c} 1),(\mathrm{c} 3)$ or $(\mathrm{c} 1),(\mathrm{c} 4)$.

(c1) For all stages $v$ with $t_{i} \leq v \leq u, b_{t_{i}}^{v}=b_{t_{i}}^{t_{i}}$, for all stages $v$ with $s \leq v \leq u$, $b_{j}^{v}=b_{j}^{s}$, and $C_{s} \uparrow_{r_{2}^{C}\left(b_{j}^{s}, n, b_{k}^{s}\right)}=C_{u} \uparrow_{r_{2}^{C}\left(b_{j}^{s}, n, b_{k}^{s}\right)}$. 
(c2) $b_{t_{i}}^{u}<_{e}^{C} n b_{k}^{s}$.

(c3) $n b_{k}^{s}<_{e}^{C} b_{t_{i}}^{u}<_{e}^{C}(n+1) b_{k}^{s}$.

(c4) $(n+1) b_{k}^{s}<_{e}^{C} b_{t_{i}}^{u}$.

When (c1) holds, if (c2) or (c3) or (c4) never appears, then $\leq_{e}^{C}$ does not satisfy totality of linear orders, we have done.

(D3) We first set a restraint $r_{4}^{C}\left(b_{t_{i}}^{u}, n, b_{k}^{s}\right) \geq r_{2}^{C}\left(b_{j}^{s}, n, b_{k}^{s}\right)$ on $C$ to preserve $\leq_{e}^{C}$ orderings in (c2) or (c3) or (c4). Now $\mathcal{N}_{e}$ has a potentially permanent witness $\left(b_{t_{i}}^{u}, b_{j}^{s}, n, b_{k}^{s}\right)$. Open cycle $i+1$ to look for new diagonalization witnesses for the active witness $\left(b_{j}^{s}, n, b_{k}^{s}\right)$.

(D4) Wait for $A \Upsilon_{t_{i}}$ to change.

(D5) Suppose that $A \Upsilon_{t_{i}}$ changes at some least stage $w \geq u>t_{i}$. That is, $w$ is the least stage $\geq u$ at which some element $x \leq t_{i}$ enumerates into $A$. If (d0) holds, stop all cycles for the active witnesses $\left(b_{j}^{s}, n, b_{k}^{s}\right)$, and proceed as in (D5.1) or (D5.2) to add dependence relations.

(d0) For all stage $v$ with $t_{i} \leq v \leq w-1, b_{t_{i}}^{v}=b_{t_{i}}^{t_{i}}$, for all stage $v$ with $s \leq v \leq w$, $b_{j}^{v}=b_{j}^{s}$, and $C_{u} \uparrow_{r_{4}^{C}\left(b_{t_{i}}^{u}, n, b_{k}^{s}\right)}=C_{w} \uparrow_{r_{4}^{C}\left(b_{t_{i}}^{u}, n, b_{k}^{s}\right)}$.

(D5.1) If $b_{t_{i}}^{w-1}<_{e}^{C} n b_{k}^{s}$ or $(n+1) b_{k}^{s}<_{e}^{C} b_{t_{i}}^{w-1}$, then add dependence relations of the form $b_{t_{i}}^{w-1}=q b_{k}^{w}$, where $q$ is properly chosen to diagonalize group orders and $q$ also satisfies the conditions asserted by the $\mathcal{Q}_{0}$-strategy and the $\mathcal{Q}_{1}$-strategy. We now state the conditions of $q$.

(d1) $q \in(n, n+1)$. Then if $\leq_{e}^{C}$ is a group order on $\mathscr{G}$, as $q \in(n, n+1)$ and $b_{k}>_{e}^{C} 0_{\mathscr{G}}$, we have $n b_{k} \leq_{e}^{C} b_{t_{i}}^{w-1}=q b_{k} \leq_{e}^{C}(n+1) b_{k}$, contradicting with the assumption for $\leq_{e}^{C}$-orderings on $b_{t_{i}}^{w-1}, n b_{k}^{s}$ and $(n+1) b_{k}^{s}$.

(d2) If $b_{t_{i}}^{w-1}=q b_{k}^{w}$, we need to find a rational-endpoint interval $\left(a_{k}^{w}, \widehat{a}_{k}^{w}\right)$ for $b_{k}^{w}$ under the $\mathcal{Q}_{1}$-strategy. Then we need to choose $q$ such that $\left(q a_{k}^{w}, q \widehat{a}_{k}^{w}\right) \subseteq$ $\left(a_{t_{i}}^{w-1}, \widehat{a}_{t_{i}}^{w-1}\right)$. As $n b_{k}^{w-1}<_{w-1} b_{t_{i}}^{w-1}<_{w-1}(n+1) b_{k}^{w-1}$, this means $n r_{k}^{w-1}<_{\mathbb{R}}$ $a_{t_{i}}^{w-1}<_{\mathbb{R}} \widehat{a}_{t_{i}}^{w-1}<_{\mathbb{R}}(n+1) r_{k}^{w-1}$. Hence, we can find infinitely many $q$ in $(n, n+1)$ with $a_{t_{i}}^{w-1}<_{\mathbb{R}} q r_{k}^{w-1}<_{\mathbb{R}} \widehat{a}_{t_{i}}^{w-1}$, and for each such a $q$, we can choose proper $a_{k}^{w}, \widehat{a}_{k}^{w}$ satisfying the conditions in the $\mathcal{Q}_{1}$-strategy. 
(d3) $q$ satisfies the conditions under the $\mathcal{Q}_{0}$-strategy, that is, $q \neq q(f, g)$ for any $f, g \in G_{w-1}$, such a $q(f, g)$ turns an inequality $f \neq g$ in $G_{w-1}$ to be an equality $f=g$ in $G_{w}$ when adding $b_{t_{i}}^{w-1}=q(f, g) b_{k}^{w}$. Note that there are only finitely many such $q(f, g)$ as $G_{w-1}$ is a finite set.

(d4) Now we have showed that there are infinitely many $q$ satisfying (d1), (d2), (d3). Our final $q$ will be the least one of them such that the numbering of $q$ (we fix an effective numbering of $\mathbb{Q}$ ) is larger than the current stage $w$.

(D5.2) If $n b_{k}^{s}<_{e}^{C} b_{t_{i}}^{w-1}<_{e}^{C}(n+1) b_{k}^{s}$, then we add dependence relations according to whether $b_{t_{i}}^{w-1}<_{w-1} b_{j}^{w-1}$ [i.e., we are in (b1)], or $b_{t_{i}}^{w-1}>_{w-1} b_{j}^{w-1}$ [i.e., we are in (b2)]. Remember that our target is still to diagonalize axioms of group orders and to make the diagonalization to be compatible with the $\mathcal{Q}_{0}$-strategy and the $\mathcal{Q}_{1}$-strategy.

(D5.2.1) If $b_{t_{i}}^{w-1}<_{w-1} b_{j}^{w-1}$. Our environment for $\leq_{\mathscr{G}}$ and $\leq_{e}^{C}$ is

$$
\begin{aligned}
& \leq_{\mathscr{G}}: n b_{k}^{w-1}<_{w-1} b_{t_{i}}^{w-1}<_{w-1} b_{j}^{w-1}<_{w-1}(n+1) b_{k}^{w-1} \\
& \leq_{e}^{C}: b_{j}^{w-1}<_{e}^{C} n b_{k}^{w-1}<_{e}^{C} b_{t_{i}}^{w-1}<_{e}^{C}(n+1) b_{k}^{w-1}
\end{aligned}
$$

Add dependence relations of the form $b_{t_{i}}^{w-1}=m_{1} b_{j}^{w}-m_{2} b_{k}^{w}$ with $m_{1}>_{\mathbb{N}} 1$. On the one hand, in order to be compatible with $\leq_{\mathscr{G}}$, (note that $b_{j}^{w}=b_{j}^{w-1}$, $\left.b_{k}^{w}=b_{k}^{w-1}\right)$, we need

$$
n b_{k}^{w-1}<_{w-1} m_{1} b_{j}^{w-1}-m_{2} b_{k}^{w-1}<_{w-1} b_{j}^{w-1}<_{w-1}(n+1) b_{k}^{w-1}
$$

Note that $b_{k}^{w-1}>\mathscr{G} 0_{\mathscr{G}}$. Then

$$
\left(m_{1}-1\right) n b_{k}^{w-1}<\mathscr{G}\left(m_{1}-1\right) b_{j}^{w-1}<\mathscr{G} m_{2} b_{k}^{w-1}
$$

and thus $\left(m_{1}-1\right) n<_{\mathbb{N}} m_{2}$.

On the other hand, if we want the dependence relation to be compatible with $\leq_{e}^{C}$, then similarly, we have

$$
b_{j}^{w-1}<_{e}^{C} n b_{k}^{w-1}<_{e}^{C} m_{1} b_{j}^{w-1}-m_{2} b_{k}^{w-1}<_{e}^{C}(n+1) b_{k}^{w-1}
$$


Note that $b_{k}^{w-1}>_{e}^{C} 0_{\mathscr{G}}$. Then

$$
m_{2} b_{k}^{w-1}<_{e}^{C}\left(m_{1}-1\right) b_{j}^{w-1}<_{e}^{C}\left(m_{1}-1\right) n b_{k}^{w-1}
$$

and thus $m_{2}<_{\mathbb{N}}\left(m_{1}-1\right) n$.

Before looking for additional conditions on $m_{1}, m_{2}$, we recall the following important lemma which will be used.

Lemma 5.1 [3] If $\left\{r_{1}, r_{2}\right\} \subseteq \mathbb{R}^{+}$is linearly independent over $\mathbb{Q}$, then for any two rational numbers $q_{1}<q_{2}$, and any integer $n \geq 1$, there are infinitely many positive integers $m_{1}, m_{2}$ such that $n\left|m_{1}, n\right| m_{2}$ and $m_{1} r_{1}-m_{2} r_{2} \in\left(q_{1}, q_{2}\right)$.

The desired $m_{1}, m_{2} \in \mathbb{N}^{+}$will satisfy the following conditions:

(f1) Choose $m_{1}, m_{2} \in \mathbb{N}$ such that $n d\left|m_{1}, n d\right| m_{2}$ and $1<_{\mathbb{N}} m_{1} \leq_{\mathbb{N}} \frac{m_{2}}{n}$, where $d$ is the product of all denominators of nonzero $q_{t_{i}}^{w-1}$ with $q_{t_{i}}^{w-1} b_{t_{i}}^{w-1}$ appearing in the $\mathbb{Q}$-linear sum of some element of $G_{w-1}$. Then $\leq_{e}^{C}$ cannot be a group order on $\mathscr{G}$.

(f2) If $b_{t_{i}}^{w-1}=m_{1} b_{j}^{w}-m_{2} b_{k}^{w}$, under the $\mathcal{Q}_{1}$-strategy, we need to find $a_{k}^{w}, \widehat{a}_{k}^{w}$, $a_{j}^{w}$ and $\widehat{a}_{j}^{w}$ such that

$r_{k}^{w}=r_{k}^{w-1} \in\left(a_{k}^{w}, \widehat{a}_{k}^{w}\right) \subseteq\left(a_{k}^{w-1}, \widehat{a}_{k}^{w-1}\right), r_{j}^{w}=r_{j}^{w-1} \in\left(a_{j}^{w}, \widehat{a}_{j}^{w}\right) \subseteq\left(a_{j}^{w-1}, \widehat{a}_{j}^{w-1}\right)$ where $\widehat{a}_{k}^{w}-a_{k}^{w}<_{\mathbb{R}} \frac{1}{2^{w}}, \widehat{a}_{j}^{w}-a_{j}^{w}<_{\mathbb{R}} \frac{1}{2^{w}}$ and

$$
\text { (अ) } \quad m_{1} r_{j}^{w}-m_{2} r_{k}^{w} \in\left(m_{1} a_{j}^{w}-m_{2} \widehat{a}_{k}^{w}, m_{1} \widehat{a}_{j}^{w}-m_{2} a_{k}^{w}\right) \subseteq\left(a_{t_{i}}^{w-1}, \widehat{a}_{t_{i}}^{w-1}\right)
$$

By Lemma 5.1, there are infinitely many natural numbers $m_{1}, m_{2}$ with $n d\left|m_{1}, n d\right| m_{2}$ such that $m_{1} r_{j}^{w}-m_{2} r_{k}^{w} \in\left(a_{t_{i}}^{w-1}, \widehat{a}_{t_{i}}^{w-1}\right)$. Then for such $m_{1}, m_{2}$, we can find proper $a_{k}^{w}, \widehat{a}_{k}^{w}, a_{j}^{w}$ and $\widehat{a}_{j}^{w}$ satisfying (h). Moreover, the calculation above shows that $m_{1} \leq_{\mathbb{N}} \frac{m_{2}}{n}$.

(f3) To make sure the $\mathbb{Q}$-linear sums of elements in $G_{w-1}$ remaining one-toone, we also require that for $i=1,2, m_{i} \neq m_{i}(f, g)$ for any $f, g \in G_{w-1}$, where adding $b_{t_{i}}^{w-1}=m_{1}(f, g) b_{j}^{w}-m_{2}(f, g) b_{k}^{w}$ entails $f=g$ at stage $w$, but $f \neq g$ at stage $w-1$. There are only finitely many such $m_{i}(f, g)$, so we can find infinitely many desired $m_{1}, m_{2}$ satisfying (f1), (f2), (f3). 
(f4) The final $m_{1}, m_{2}$ will satisfy (f1), (f2), (f3) with $m_{1}, m_{2}>w$ and $\left\langle m_{1}, m_{2}\right\rangle$ least.

(D5.2.2) If $b_{t_{i}}^{w-1}>_{w-1} b_{j}^{w-1}$. Our environment for $\leq_{\mathscr{G}}$ and $\leq_{e}^{C}$ is

$$
\begin{aligned}
& \leq_{\mathscr{G}}: n b_{k}^{w-1}<_{w-1} b_{j}^{w-1}<_{w-1} b_{t_{i}}^{w-1}<_{w-1}(n+1) b_{k}^{w-1} \\
& \leq_{e}^{C}: n b_{k}^{w-1}<_{e}^{C} b_{t_{i}}^{w-1}<_{e}^{C}(n+1) b_{k}^{w-1}<_{e}^{C} b_{j}^{w-1}
\end{aligned}
$$

Add dependence relations of the form $b_{t_{i}}^{w-1}=m_{1} b_{k}^{w}-m_{2} b_{j}^{w}$. We choose $m_{1}, m_{2}$ such that substituting the relation into $\leq_{\mathscr{G}}$ holds, that is,

$$
n b_{k}^{w-1}<_{w-1} b_{j}^{w-1}<_{w-1} m_{1} b_{k}^{w-1}-m_{2} b_{j}^{w-1}<_{w-1}(n+1) b_{k}^{w-1}
$$

Then

$$
\begin{aligned}
\left(m_{2}+1\right)\left[m_{1}-(n+1)\right] b_{k}^{w-1} & <_{w-1}\left(m_{2}+1\right) m_{2} b_{j}^{w-1} \\
& <_{w-1} m_{2} m_{1} b_{k}^{w-1}
\end{aligned}
$$

So $\left(m_{2}+1\right)\left[m_{1}-(n+1)\right]<_{\mathbb{N}} m_{2} m_{1}$, and thus $m_{1}<_{\mathbb{N}}(n+1)\left(m_{2}+1\right)$.

Now under the conditions $m_{1}<_{\mathbb{N}}(n+1)\left(m_{2}+1\right)$ and $(n+1) \mid m_{1}, \frac{m_{1}}{(n+1)}<_{\mathbb{N}}$ $m_{2}+1$, that is, $\frac{m_{1}}{(n+1)} \leq_{\mathbb{N}} m_{2}$. Then $\leq_{e}^{C}$ is not a group order on $\mathscr{G}$. Indeed, suppose that $\leq_{e}^{C}$ is a group order on $\mathscr{G}$, then

$$
\begin{aligned}
0_{\mathscr{G}}<_{e}^{C} n b_{k}^{w-1}<_{e}^{C} b_{t_{i}}^{w-1} & =m_{1} b_{k}^{w-1}-m_{2} b_{j}^{w-1} \\
& \leq_{e}^{C} m_{1} b_{k}^{w-1}-\frac{m_{1}}{n+1} b_{j}^{w-1} \\
& =\frac{m_{1}}{n+1}\left((n+1) b_{k}^{w-1}-b_{j}^{w-1}\right)<_{e}^{C} 0_{\mathscr{G}}
\end{aligned}
$$

We now determine $m_{1}, m_{2}$ :

(g1) Choose $m_{1}, m_{2} \in \mathbb{N}$ such that $(n+1) d\left|m_{1},(n+1) d\right| m_{2}$ and $\frac{m_{1}}{(n+1)} \leq_{\mathbb{N}} m_{2}$, where $d$ is the same as in (f1).

(g2) Assume that $b_{t_{i}}^{w-1}=m_{1} b_{k}^{w}-m_{2} b_{j}^{w}$. We need to assign rational-endpoint intervals for $b_{t_{i}}^{w-1}$ at stage $w$, that is, find $a_{k}^{w}, \widehat{a}_{k}^{w}, a_{j}^{w}$ and $\widehat{a}_{j}^{w}$ the same as in (f2) but with the condition (o) changed to

(A) $\quad m_{1} r_{k}^{w}-m_{2} r_{j}^{w} \in\left(m_{1} a_{k}^{w}-m_{2} \widehat{a}_{j}^{w}, m_{1} \widehat{a}_{k}^{w}-m_{2} a_{j}^{w}\right) \subseteq\left(a_{t_{i}}^{w-1}, \widehat{a}_{t_{i}}^{w-1}\right)$ 
According to Lemma 5.1, there are infinitely many natural numbers $m_{1}, m_{2}$ with $(n+1) d\left|m_{1},(n+1) d\right| m_{2}$ such that $m_{1} r_{k}^{w}-m_{2} r_{j}^{w} \in\left(a_{t_{i}}^{w-1}, \widehat{a}_{t_{i}}^{w-1}\right)$. Then we can choose proper $\left(a_{k}^{w}, \widehat{a}_{k}^{w}\right) \subseteq\left(a_{k}^{w-1}, \widehat{a}_{k}^{w-1}\right)$, and proper $\left(a_{j}^{w}, \widehat{a}_{j}^{w}\right) \subseteq$ $\left(a_{j}^{w-1}, \widehat{a}_{j}^{w-1}\right)$ satisfying $(\mathbf{A})$. Then $\frac{m_{1}}{(n+1)} \leq_{\mathbb{N}} m_{2}$ automatically holds.

(g3) The same as in (f3), we require that the choice of $m_{1}$ and $m_{2}$ preserves the one-to-one assignment of $\mathbb{Q}$-linear sums to elements in $G_{w-1}$.

(g4) The final $m_{1}$ and $m_{2}$ satisfy (g1), (g2), (g3), $m_{1}>w, m_{2}>w$ with $\left\langle m_{1}, m_{2}\right\rangle$ least.

This ends the description of the basic $\mathcal{N}_{e^{-}}$strategy.

The interactions between two $\mathcal{N}$-strategies are finite as in [3]. They both set $C$-restraints, so there is no confliction in building $C$; the confliction is in choosing witnesses.

Suppose that $\mathcal{N}_{e}$ choose an active witness $\left(b_{j}^{s}, n, b_{k}^{s}\right)$ at stage $s$, and then add a diagonalization witness $b_{t}^{t}$ at an odd stage $t>s$. To diagonalize axioms of group orders, $\mathcal{N}_{e}$ may add dependence relations to change $b_{t}^{t}$ at stages $>t$. However, some $\mathcal{N}_{i}$ may find an active witness $\left(b_{j^{\prime}}^{u}, n^{\prime}, b_{k^{\prime}}^{u}\right)$ at stage $u>t$ with $b_{j^{\prime}}^{u}=b_{t}^{t}$, and then add a diagonalization witness $b_{w}^{w}$ at an odd stage $w>u$. To preserve its active witness, $\mathcal{N}_{i}$ requires $b_{t}^{t}$ keeping the same at stages $>u$.

The solution is a priority argument. When $e<i$,

(1) if $\mathcal{N}_{e}$ acts first to add dependence relations, it changes $b_{t}^{t}$ to initialize $\mathcal{N}_{i}$ 's active witnesses $\left(\mathcal{N}_{e}\right.$ is satisfied and cannot injure $\mathcal{N}_{i}$ later, $\mathcal{N}_{i}$ will choose new active witnesses at later stages);

(2) if $\mathcal{N}_{i}$ acts first, it adds dependence relations on $b_{w}^{w}$ and hence does not change $b_{j^{\prime}}^{u}\left(=b_{t}^{t}\right)$, so no confliction occurs.

When $i<e$,

- if $\mathcal{N}_{i}$ finds its active witness, say $\left(b_{j^{\prime}}^{u}, n^{\prime}, b_{k^{\prime}}^{u}\right)$, at stage $u>t$ with $b_{j^{\prime}}^{u}=b_{t}^{t}$, it cancels $\mathcal{N}_{e}$ 's diagonalization witness $b_{t}^{t}$ to avoid potential conflictions $\left(\mathcal{N}_{e}\right.$ will find its diagonalization witness $b_{t^{\prime}}^{t^{\prime}}$ with $t^{\prime}$ big at later odd stages). 
$\mathcal{P}$-requirements are purely set-theoretic requirements to ensure the noncomputability of $C$. Each $\mathcal{P}_{e}$ is positive on $C$ and acts at most once to enumerate a permitted witness $y \in W_{e}$ into $C$. So $\mathcal{P}_{e}$ injures negative requirements with lower priority which may set restraints on $C$ finitely often.

\subsection{Construction}

$\mathcal{Q}, \mathcal{Q}_{0}$ and $\mathcal{Q}_{1}$ are global requirements. The priority of other requirements is as follows:

$$
\mathcal{N}_{0} \prec \mathcal{P}_{0} \prec \cdots \mathcal{N}_{e} \prec \mathcal{P}_{e} \prec \cdots
$$

The construction mainly splits into two parts. One is set-theoretic constructions, that is, building set $C$. The other is algebraic constructions, that is, adding dependence relations to defeat $\leq_{e}^{C}$ to be a group order on $\mathscr{G}$, and then constructing $\mathscr{G}$ and $\leq_{\mathscr{G}}$ according to whether some dependence relations are added or not.

\section{Construction.}

Stage 0. Let $C_{0}=\varnothing$. Build $G_{0}$ and $\leq_{0}$ by the $\mathcal{Q}_{0}$-strategy in Section 5.1 .2 and the $\mathcal{Q}_{1}$-strategy in Section 5.1 .3 respectively.

Stage $s>0$.

Step 1. Find the highest priority requirement, say $Q$, among

$$
\mathcal{N}_{0} \prec \mathcal{P}_{0} \prec \cdots \mathcal{N}_{s} \prec \mathcal{P}_{s}
$$

which requires attention (such a requirement exists since $\mathcal{P}_{s}$ requires attention at stage $s$ ). Act correspondingly and initialize all strategies $\succ Q$. Goto Step 2 if no dependence relations are added; goto Step 3 otherwise.

Say that $\mathcal{P}_{e}$ is satisfied at stage $s$ if $W_{e, s} \cap C_{s} \neq \varnothing$, where $W_{e, s}$ is the set of all elements enumerated into $W_{e}$ by stage $s$. Say that $\mathcal{P}_{e}$ requires attention at stage $s$ if it is unsatisfied and one of the following (P1) and (P2) happens. Act accordingly.

(P1) $\mathcal{P}_{e}$ has no cycles opened at stage $s$.

Action: Open cycle 0 by appointing a large witness $y_{0}$, and start to wait for $y_{0}$ enumerating into $W_{e}$. Proceed as in the basic $\mathcal{P}_{e^{-}}$strategy. 
(P2) $\mathcal{P}_{e}$ has a previous witnesses $y_{k} \in W_{e, s_{k}}$, cycle $k+1$ is opened at stage $s_{k}$ and cycle $k$ started to wait for $A_{s_{k}} \Upsilon_{y_{k}}$-changes at stage $s_{k}$. Now $A_{s_{k}} \Upsilon_{y_{k}}=$ $A_{s-1} \Upsilon_{y_{k}} \neq A_{s} \Upsilon_{y_{k}}$ and stage $s \geq s_{k}$ is the first stage at which some cycles of $\mathcal{P}_{e}$

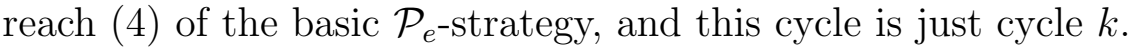

Action: Put $y_{k}$ into $C_{s}$, close all opened cycles of $\mathcal{P}_{e}$. Declare that $\mathcal{P}_{e}$ is satisfied.

Say that $\mathcal{N}_{e}$ is satisfied if we add dependence relations between some active witness and some diagonalization witness. That is, we have finished the following actions.

(1) We find some active witness, say $\left(b_{j}^{s}, n, b_{k}^{s}\right)$, and assign $C$-restraints to protect this active witness.

(2) We arrive at some cycle $i$ for $\left(b_{j}^{s}, n, b_{k}^{s}\right)$ with some diagonalization witness $b_{t_{i}}^{t_{i}}$ and set $C$-restraints to protect potentially permanent witness $\left(b_{t_{i}}^{u}, b_{j}^{s}, n, b_{k}^{s}\right)$ at some stage $u \geq t_{i}$.

(3) $A \Upsilon_{t_{i}}$ changes after stage $u$, and we add dependence relations between diagonalization witness and active witness.

In this case, $\leq_{e}^{C}$ is not an order on $\mathscr{G}$.

Say that $\mathcal{N}_{e}$ requires attention at stage $s$ if it is not satisfied at stage $s$, and one of the following holds at stage $s$. Act correspondingly.

(N1) Some active witness appears at stage $s$.

Action: First, set $C$-restraints to protect this active witness. Second, if some

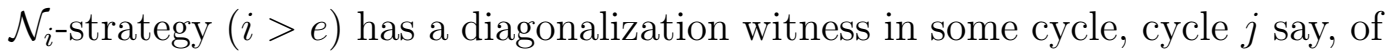
some active witness which occurs in the active witness of $\mathcal{N}_{e}$, then cancel the diagonalization witness at cycle $k$, where $k \geq j$, of $\mathcal{N}_{i}$ 's active witness.

(N2) $\mathcal{N}_{e}$ has an active witness and no cycles opened for this active witness at stage $s$.

Action: Open cycle 0 of the active witness to find diagonalization witnesses. 
(N3) $\mathcal{N}_{e}$ has some active witness, and some cycle $i$ is opened for this active witness. No diagonalization witness is found in cycle $i$ at stage $s$, and $s$ is odd.

Action: Set the diagonalization witness $b_{t_{i}}^{t_{i}}=b_{s}^{s}$ at cycle $i$ of the active witness, and order $b_{s}^{s}$ with the active witness in (b1) or (b2) as in the basic $\mathcal{N}_{e}$-strategy.

(N4) $\mathcal{N}_{e}$ has some active witness, and some cycle $i$ with uncanceled diagonalization witness $b_{t_{i}}^{t_{i}}$ is opened for this active witness. Now one of the ordering relations between the active witness and $b_{t_{i}}^{t_{i}}$ in (c2) or (c3) or (c4) appears at stage $s$.

Action: Set $C$-restraints to protect the potentially permanent witnesses at cycle $i$. Open cycle $i+1$ for the active witness to seek for new diagonalization witnesses and simultaneously, wait for $A \uparrow_{t_{i}}$ to change.

(N5) $\mathcal{N}_{e}$ has a potentially permanent witnesses with diagonalization witness $b_{t_{i}}^{t_{i}}$ in cycle $i$, and some $x \leq t_{i}$ first enumerates into $A$ at stage $s$.

Action: For such a least cycle $i$, add dependence relations on $b_{t_{i}}^{t_{i}}$ according to the basic $\mathcal{N}_{e}$-strategy, and then stop all cycles of $\mathcal{N}_{e}$. Declare that $\mathcal{N}_{e}$ is satisfied.

Step 2. Build $G_{s}$ and $\leq_{s}$ respectively according to the $\mathcal{Q}_{0}$-strategy in Section 5.1.2 and the $\mathcal{Q}_{1}$-strategy in Section 5.1 .3 with no dependence relations added.

Step 3. Build $G_{s}$ and $\leq_{s}$ respectively according to the $\mathcal{Q}_{0}$-strategy in Section 5.1.2 and the $\mathcal{Q}_{1}$-strategy in Section 5.1 .3 with the added dependence relation.

This ends the stage $s$ of the construction.

\subsection{Verification}

Lemma 5.2 For each requirement, $Q$ say,

(1) $Q$ can be initialized at most finitely often;

(2) $Q$ acts at most finitely often and is satisfied;

(3) Q initializes strategies with lower priority finitely often. 
Proof: We will show that for each $e,(1)-(3)$ hold for all $\mathcal{N}_{e}, \mathcal{P}_{e}$. We will prove the lemma by induction. For $e=0 . \mathcal{N}_{0}$ has the highest priority, it cannot be initialized. (1) holds. We now show that it acts finitely often and is satisfied.

If $\leq_{0}^{C}$ has no active witnesses during the construction, then by Lemma 3.9 in [3], either $\leq_{0}^{C}$ is not a group order, or $\leq_{0}^{C}=\leq_{\mathscr{G}}$ or $\leq_{0}^{C}=\leq_{\mathscr{G}}^{*} . \mathcal{N}_{0}$ is satisfied.

Suppose that there is some active witness $\left(b_{j}^{s}, n, b_{k}^{s}\right)$ for $\mathcal{N}_{0}$ at stage $s$. Then $\mathcal{N}_{0}$ acts to protect $\left(b_{j}^{s}, n, b_{k}^{s}\right)$ by setting $C$-restraints, and initializes all strategies with lower priority.

Under the basic $\mathcal{N}_{0}$-strategy, it starts by opening cycle 0 , that is, by waiting for a diagonalization witness $b_{t_{0}}^{t_{0}}$ at some odd stage $t_{0}>s$ and then waiting for

$$
\left(\boldsymbol{中}_{0}\right): \quad b_{t_{0}}^{t_{0}}<_{0}^{C} n b_{k}^{s} \vee n b_{k}^{s}<_{0}^{C} b_{t_{0}}^{t_{0}}<_{0}^{C}(n+1) b_{k}^{s} \vee(n+1) b_{k}^{s}<_{0}^{C} b_{t_{0}}^{t_{0}} .
$$

In general, cycle $i>0$ works as follows.

(i) Wait for a diagonalization witness $b_{t_{i}}^{t_{i}}$ at some odd stage $t_{i}>u_{i-1}>t_{i-1}$ and then wait for

$$
\left(\boldsymbol{\leftrightarrow}_{i}\right): \quad b_{t_{i}}^{t_{i}}<_{0}^{C} n b_{k}^{s} \vee n b_{k}^{s}<_{0}^{C} b_{t_{i}}^{t_{i}}<_{0}^{C}(n+1) b_{k}^{s} \vee(n+1) b_{k}^{s}<_{0}^{C} b_{t_{i}}^{t_{i}} .
$$

If $\left(\boldsymbol{\sim}_{i}\right)$ never happens, then $\mathcal{N}_{0}$ is satisfied because $\leq_{0}^{C}$ does not satisfy the axioms of linear orders.

(ii) If $\left(\boldsymbol{\leftrightarrow}_{i}\right)$ happens at some stage $u_{i}>t_{i}$. Open cycle $i+1$ and simultaneously wait for $A \uparrow_{t_{i}}$ to change.

- If $A \uparrow_{t_{i}}$ changes at some stage $w_{i}>u_{i}$, add dependence relations between $b_{t_{i}}^{t_{i}}$ and $\left(b_{j}^{s}, n, b_{k}^{s}\right)$. Close all cycles of $\left(b_{j}^{s}, n, b_{k}^{s}\right)$, and $\mathcal{N}_{0}$ is satisfied.

If there is a cycle $i$ which waits at $\left(\boldsymbol{A}_{i}\right)$ forever, then cycle $i+1$ is never opened. In this case, $\leq_{0}^{C}$ is not a linear order and thus $\mathcal{N}_{0}$ is satisfied. So assume that no cycles $i$ wait at $\left(\boldsymbol{A}_{i}\right)$ forever.

Suppose that each cycle $i$ is not closed, then each cycle $i$ waits for $A \uparrow_{t_{i}}$ to change forever, that is, $A_{u_{i}} \Upsilon_{t_{i}}=A \Upsilon_{t_{i}}$. Note that $s \leq t_{i}<u_{i}<t_{i+1}$ for all $i$. Now we can compute $A$ as follows: for any $x$, find the least $t_{i}$ with $x<t_{i}$, then $x \in A$ iff $x \in A_{u_{i}}$. 
As $A$ is assumed to be noncomputable, there is a cycle $i$ which provides a diagonalization witness $b_{t_{i}}^{t_{i}}$, and $A_{u_{i}} \Upsilon_{t_{i}}$ changes later, say at stage $t^{\prime} \geq u_{i}$. Then we add dependence relations between $b_{t_{i}}^{t_{i}}$ and $\left(b_{j}^{s}, n, b_{k}^{s}\right)$ at stage $t^{\prime}$, and $\mathcal{N}_{0}$ is satisfied.

Hence, in all cases above, $\mathcal{N}_{0}$ acts finitely many times and is satisfied at end. (2) and (3) hold.

Let $s_{0}$ be the least stage such that $\mathcal{N}_{0}$ never acts later, then $\mathcal{P}_{0}$ is not injured after stage $s_{0}$. Let $t_{0} \geq s_{0}$ be the least stage such that cycle 0 of $\mathcal{P}_{0}$ is opened. As long as $\mathcal{P}_{0}$ is not satisfied, the basic $\mathcal{P}_{0}$-strategy will open cycles, say $k$, by picking a new witness $y_{k}$ at cycle $k$ and starting to wait for $y_{k} \in W_{0}$; when $y_{k} \in W_{0}, y_{k}$ is enumerated into $C$ at some stage $s$ only if $A_{s} \uparrow_{y_{k}} \neq A_{s-1} \Upsilon_{y_{k}}$. As $A$ is noncomputable, if such witnesses eventually enumerate into $W_{0}$, then this $A$-permitting happens at certain cycles. So $\mathcal{P}_{0}$-strategy can only open finitely many cycles. That is, $\mathcal{P}_{0}$ acts finitely often after stage $s_{0}$.

As long as $\mathcal{P}_{0}$ is not satisfied automatically, there is a cycle $k$ such that either $y_{k}$ is never put into $C$ in which case $y_{k} \notin W_{0}$ or $y_{k}$ is put into $C$ in which case $y_{k} \in W_{0}$. In both cases, $W_{0} \neq \bar{C}$. So $\mathcal{P}_{0}$ is satisfied after finitely many actions, thus (2) and (3) hold.

Now assume that the lemma holds for all $\mathcal{N}_{i}, \mathcal{P}_{i}$ with $i<e$. By induction hypothesis, there is a stage $s_{e}$ such that $\mathcal{N}_{i}, \mathcal{P}_{i}$ with $i<e$ are satisfied at stage $s_{e}$. Then $\mathcal{N}_{e}$ has the highest priority after stage $s_{e}$. Similar to case $e=0,(1)-(3)$ hold for $\mathcal{N}_{e}$ and $\mathcal{P}_{e}$.

Lemma 5.3 $C \leq_{T} A$.

Proof: During the construction, only permitted elements can be enumerated into $C$. That is, $x \in C_{s}$ only if there is some small element $z \leq x$ such that $z \in A_{s}-A_{s-1}$. To decide whether $x \in C$ or not, by using $A$, find a least stage $s$ such that for all $z \leq x, z \in A \Leftrightarrow z \in A_{s}$. Then $x \in C \Leftrightarrow x \in C_{s}$.

The differences between Theorem 5.1 and Theorem 1.17 are that elements enumerated into $C$ are permitted by $A$ and that the diagonalization witnesses are permitted by $A$. We now show that for each $i$, the approximation basis element $b_{i}^{s}$ only changes finitely often. Then $\lim _{s} b_{i}^{s}$ exists, and thus we can satisfy $\mathcal{Q}_{0}$ and $\mathcal{Q}_{1}$ the same as in Theorem 1.17. 
Lemma 5.4 For each $i, b_{i}=\lim _{s} b_{i}^{s}$ exists.

Proof: For each $i$, consider $b_{i}^{i}$. It may change at later stages only if $b_{i}^{i}$ is chosen as a diagonalization witness in some cycle of some active witnesses for some $\mathcal{N}$ requirements. When $b_{i}^{i}$ is not a diagonalization witness, it never changes. So $b_{i}=b_{i}^{s}$ for all $s \geq i$.

Assume that $b_{i}^{i}$ is a diagonalization witness. $b_{i}^{i}$ may be changed at most once when adding dependence relations on $b_{i}^{s-1}=b_{i}^{i}$ at some large stage $s$ with $A_{s-1} \Upsilon_{i} \neq A_{s} \Upsilon_{i}$. Then we will define a new basis approximation element $b_{i}^{s}$, and $b_{i}=b_{i}^{s}$ never changes later since $b_{i}^{s}$ is not a diagonalization witness any more. So the approximations $b_{i}^{s}(s \in \mathbb{N})$ of $b_{i}$ change at most once. Thus, $b_{i}=\lim _{s} b_{i}^{s}$ exists.

Lemma $5.5 B=\left\{b_{i}: i \in \mathbb{N}\right\} \leq_{T} A$.

Proof: For any $g \in G=\cup_{s} G_{s}$, the domain of $\mathscr{G}$. If it is not $b_{i}^{s}$ with $s \geq i$ when first added into $G$, then $g \notin B$. Otherwise, assume that $g=b_{i}^{s}$ is first added at stage $s$.

Case 1. $i=s$. If $i$ is even, then $b_{i}^{i}$ never changes later and $g=b_{i}=b_{i}^{i} \in B$. If $i$ is odd and $g=b_{i}^{i}$ is not a diagonalization witness for $\mathcal{N}$-requirements, then $g \in B$. If $g$ is a diagonalization witness: first, by using $A$, find the least stage $t_{0}$ such that $A_{t_{0}} \Upsilon_{i}=A \Upsilon_{i}$; second, check whether we add dependence relations on $b_{i}^{i}$ during stages in $\left(i, t_{0}\right]$. If we add dependence relations and redefine approximations for $b_{i}$, then $g \neq b_{i}$ and $g \notin B$. If no dependence relations are added on $b_{i}^{i}$, that is, $b_{i}^{t_{0}}=b_{i}^{i}$, then $g=b_{i} \in B$.

Case 2. $i<s$. Then $i$ is odd and we change $b_{i}^{s-1}$ at stage $s$; in this case, $g=b_{i}^{s}$ is never changed later. So $g=b_{i} \in B$.

By Lemma 5.2, all $\mathcal{P}_{e}, \mathcal{N}_{e}$ hold. Then $C$ is not computable; and for all $e$, either $\leq_{e}^{C}$ is not a group order on $\mathscr{G}$ or $\leq_{e}^{C}=\leq_{\mathscr{G}}$ or $\leq_{e}^{C}=\leq_{\mathscr{G}}^{*}$, that is, every $C$-computable order on $\mathscr{G}$ is computable. So $\mathscr{G}$ has no incomputable orders of degree $\leq \operatorname{deg}(C)$.

By Lemma 5.3, $C \leq_{T} A$. By Lemma 5.5, $\mathscr{G}$ has an $A$-computable basis, so $\mathscr{G}$ has an order of degree $\geq \operatorname{deg}(A)$. Moreover, as $\mathscr{G}$ has no orders of degree $C, C<_{T} A$.

This completes the proof of Theorem 5.1. 


\section{Part III}

\section{Reverse mathematics, abelian groups and modules}




\section{Chapter 6}

\section{Reverse mathematics and divisible abelian groups}

In this chapter, we will examine the proof-theoretic strength of several classical theorems related to countable divisible abelian groups in the program of reverse mathematics. For more background on abelian groups, refer to [29].

Definition $6.1\left(\mathrm{RCA}_{0}\right)$ Let $G$ be an abelian group. $G$ is divisible if for any $g \in G$ and nonzero $n \in \mathbb{N}$, there is a $g^{\prime} \in G$ such that $g=n g^{\prime}$, and we say $g$ is divisible by $n$ in $G$.

In this chapter, groups always mean abelian groups.

\subsection{Divisible subgroups}

Definition $6.2\left(\mathrm{RCA}_{0}\right)$ Let $I$ be an abelian group. I is called injective if for any monomorphism $\mu: A \rightarrow B$ and any homomorphism $\varphi: A \rightarrow I$, there exists a homomorphism $\psi: B \rightarrow I$ such that $\varphi=\psi \mu$.

We now state a theorem related to injective abelian groups in reverse mathematics, as we will use it later to prove Theorem 6.2.

Theorem 6.1 [26] The following are equivalent over $\mathrm{RCA}_{0}$.

(1) $\mathrm{ACA}_{0}$

(2) Every divisible abelian group is injective. 
Friedman, Simpson and Smith [26] showed that $\mathrm{ACA}_{0}$ proves the statement that "every divisible subgroup of an abelian group $G$ is a direct summand of $G$ ". We are interested in the reversal part of this statement. We will complete the work above of Friedman, Simpson and Smith, by proving the following theorem.

Theorem 6.2 Over $\mathrm{RCA}_{0}$, the following are equivalent:

(1) $\mathrm{ACA}_{0}$

(2) Every divisible subgroup of an abelian group is a direct summand.

Proof: $\quad(1) \Rightarrow(2)$ was proved by Friedman, Simpson and Smith in their paper [26] (Lemma 6.2). For completeness, we provide a proof here.

Let $G$ be an abelian group and $D$ be a divisible subgroup of $G$. As $\mathrm{ACA}_{0}$ proves that every divisible group is injective, $D$ is injective. Consider the following diagram with $1_{D}: D \rightarrow D$ the identity homomorphism and $\mu: D \rightarrow G$ the identical embedding:

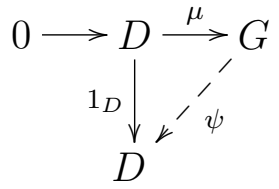

Then there is surjective homomorphism $\psi$ with $1_{D}=\psi \mu$, and the kernel of $\psi$, $\operatorname{ker}(\psi)$, is $\{g \in G: \psi(g)=0\}$, and $G=\operatorname{ker}(\psi) \oplus i m(\psi)=\operatorname{ker}(\psi) \oplus D$. This completes the proof of $(1) \Rightarrow(2)$.

$(2) \Rightarrow(1)$. Fix a one-to-one function $f: \mathbb{N} \rightarrow \mathbb{N}$. Let $G$ be the divisible abelian group $\bigoplus_{n \in \mathbb{N}} \mathbb{Q} x_{n}$, where elements of $G$ are finite $\mathbb{Q}$-linear sums of elements from $\left\{x_{n}: n \in \mathbb{N}\right\}$. For $m \in \mathbb{N}$, let $x_{m}^{\prime}=x_{2 f(m)}+m x_{2 f(m)+1}$. Then $\bigoplus_{m \in \mathbb{N}} \mathbb{Q} x_{m}^{\prime}$ is a divisible subgroup of $G$, denoted by $D$. $D$ exists in $\mathrm{RCA}_{0}$.

By our assumption (2), $D$ is a direct summand of $G$. So there is a subgroup $K$ of $G$ such that $G=D \oplus K$. For any $n, x_{2 n}$ and $x_{2 n+1}$ now can be uniquely written as

- $x_{2 n}=y_{2 n}+z_{2 n}$,

- $x_{2 n+1}=y_{2 n+1}+z_{2 n+1}$, 
where $y_{2 n}, y_{2 n+1} \in D$ and $z_{2 n}, z_{2 n+1} \in K$.

Recall that any element in $D$ can be expressed as a unique $\mathbb{Q}$-linear sum of elements in $\left\{x_{m}^{\prime}: m \in \mathbb{N}\right\}$. Let $I_{n}$ be the finite set consisting of all indices $m$ where $x_{m}^{\prime}$ appears in the expression of $y_{2 n}$ or $y_{2 n+1}$. Then

$$
n \in \operatorname{range}(f) \Leftrightarrow \exists m \in I_{n}[f(m)=n] .
$$

We only need to show that if $n=f\left(m_{0}\right)$, then $m_{0} \in I_{n}$. As

$$
x_{m_{0}}^{\prime}=x_{2 n}+m_{0} x_{2 n+1}=\left(y_{2 n}+m_{0} y_{2 n+1}\right)+\left(z_{2 n}+m_{0} z_{2 n+1}\right)
$$

and the expressions of elements in $D$ as linear combinations of elements in $\left\{x_{m}^{\prime}: m \in\right.$ $\mathbb{N}\}$ are unique, we have $x_{m_{0}}^{\prime}=y_{2 n}+m_{0} y_{2 n+1}$. Then $x_{m_{0}}^{\prime}$ appears in the expression of $y_{2 n}$ or $y_{2 n+1}$, i.e., $m_{0} \in I_{n}$.

Now range $(f)$ exists, by the $\Delta_{1}^{0}$-comprehension.

Classically, every abelian group has the unique largest divisible subgroup which is the union of all divisible subgroups. This definition is $\Sigma_{1}^{1}$. We will show that the existence of the largest divisible subgroup of an abelian group is equivalent to $\Pi_{1}^{1}-\mathrm{CA}_{0}$ over $\mathrm{RCA}_{0}$. In order to prove the reversal part, we need the following wellknown theorem of Friedman, Simpson and Smith. Recall that an abelian group is reduced if it has no nontrivial divisible subgroups.

Theorem 6.3 [26] Over $\mathrm{RCA}_{0}$, the following are equivalent.

(1) $\Pi_{1}^{1}-\mathrm{CA}_{0}$

(2) Every abelian group is a direct sum of a divisible subgroup and a reduced subgroup.

However, for the special case of torsion-free abelian groups, as shown in Theorem 6.4 below, we show that the existence of the largest divisible subgroup is equivalent to the arithmetical comprehension.

Theorem 6.4 Over $\mathrm{RCA}_{0}$, the following are equivalent:

(1) $\mathrm{ACA}_{0}$ 
(2) Every torsion-free abelian group has the largest divisible subgroup.

Proof: $\quad(1) \Rightarrow(2)$. Let $G$ be a countable abelian group. Let

$$
D=\left\{g \in G: \forall n>0 \exists g^{\prime} \in G\left[g=n g^{\prime}\right]\right\}
$$

$D$ exists by arithmetical comprehension.

We will show that $D$ is the largest divisible subgroup of $G$. Clearly, the zero $0_{G}$ of $G$ is in $D$. For any $m$, if $m$ divides $g_{n_{1}}$ and $g_{n_{2}}$ in $G$, then $m$ divides $g_{n_{1}}-g_{n_{2}}$ in $G$. Thus, $D$ is a subgroup of $G$.

We now show that $D$ is divisible. For any $g \in D, g$ is divisible by all nonzero natural numbers in $G$. So for any $m \geq 1$, there is a $h_{m} \in G$ such that $g=m h_{m}$. We claim that $h_{m} \in D$. Then $g$ is also divisible by all nonzero natural numbers in $D$. Thus, $D$ is a divisible subgroup of $G$.

Claim. $h_{m} \in D$.

Proof of the claim.

Fix $k \geq 1$. As $g=m h_{m}=m k h_{m k}, m\left(h_{m}-k h_{m k}\right)=0_{G}$. Here, the index $m k$ of $h_{m k}$ is the multiplication of $m$ and $k$. As $m \neq 0$ and $G$ is torsion-free, $h_{m}-k h_{m k}=0_{G}$, i.e., $h_{m}=k h_{m k}$. So $h_{m}$ is divisible by $k$ in $G$. We have showed that $h_{m}$ is divisible by all nonzero natural numbers in $G$. By definition, $h_{m} \in D$.

This ends the proof of the claim.

Now we show that $D$ is the largest divisible subgroup of $G$. Take any divisible subgroup $A$ of $G$. For any $a \in A$ and $n>0$, there is an $a^{\prime} \in A$ such that $a=n a^{\prime}$. Since $a^{\prime} \in G, a \in D$. Thus $A \subseteq D$.

(2) $\Rightarrow(1)$. Let $f: \mathbb{N} \rightarrow \mathbb{N}$ be a one-to-one function. Consider the torsion-free abelian group $\bigoplus_{n \in \mathbb{N}} \mathbb{Q} x_{n}$, denoted by $G$. Again, elements of $G$ are just finite $\mathbb{Q}$-linear sums of elements in $\left\{x_{n}: n \in \mathbb{N}\right\}$.

Let $H$ be the set of all elements $\sum_{n<m} a_{n} x_{n}$ of $G$ such that for each $n$, if the denominator of $a_{n}$ has a prime factor $p_{l}$, then $f(l) \neq n$. One can check that $H$ is a subgroup of $G$ and that $H$ exists in $\mathrm{RCA}_{0}$.

By (2), $H$ has the largest divisible subgroup, say $D$. Let

$$
D^{\prime}=\left\{g \in H: \forall n>0 \exists g^{\prime} \in H\left[g=n g^{\prime}\right]\right\} .
$$


From the proof of the direction $(1) \Rightarrow(2)$, we see that $D^{\prime}$ is a divisible subgroup of $H$ and that $D \subseteq D^{\prime}$. Then by the choice of $D, D^{\prime}=D$.

We now show $n \in \operatorname{range}(f) \Leftrightarrow x_{n} \notin D$.

- If $n=f(m)$, then $\frac{1}{p_{m}} x_{n} \notin H$. Note that the only element $y$ in $G$ with $x_{n}=p_{m} y$ is $\frac{1}{p_{m}} x_{n}$. Thus no elements $y$ in $H$ satisfy $x_{n}=p_{m} y$, and $x_{n} \notin D$.

- If $n \notin \operatorname{range}(f)$, then $\frac{1}{k} x_{n} \in H$ for any $k \geq 1$. Thus $x_{n} \in D$.

By $\Delta_{1}^{0}$-comprehension, range $(f)$ exists.

Corollary 6.1 Over $\mathrm{RCA}_{0}$, the following are equivalent:

(1) $\Pi_{1}^{1}-\mathrm{CA}_{0}$

(2) Every abelian group has the largest divisible subgroup.

(3) Every abelian group is a direct sum of a divisible subgroup and a reduced subgroup.

Proof: By definition, (1) $\Rightarrow(2)$. By Theorem 6.3 of Friedman, Simpson and Smith, $(3) \Rightarrow(1)$. We now show $(2) \Rightarrow(3)$.

Assume (2). Then every torsion-free abelian group has the largest divisible subgroup. By Theorem 6.4, this implies $\mathrm{ACA}_{0}$. Now let $G$ be an abelian group. Again, by (2), it has the largest divisible subgroup, say D. By Theorem 6.2, $\mathrm{ACA}_{0}$ proves $G=D \oplus R$ for some subgroup $R$. As $D$ is the largest divisible subgroup, $R$ is reduced. Hence, (3) holds.

\subsection{Two decomposition theorems}

Definition 6.3 $\left(\mathrm{RCA}_{0}\right)$ An abelian group is primary if there is a prime $p$ such that its elements have orders a power of $p$.

For each $i \in \mathbb{N}, p_{i}$ is the $i$-th prime number, starting with $p_{0}=2$. Let $P$ be the set of all prime numbers.

Theorem 6.5 Over $\mathrm{RCA}_{0}$, the following are equivalent: 
(1) $\mathrm{ACA}_{0}$

(2) Every nontrivial torsion group, say $T$, is a direct sum of primary subgroups. That is, there is a set $I \subseteq P$ such that $T=\bigoplus_{p \in I} T_{p}$, where for each $p \in P$, $T_{p}=\left\{g \in T: \exists n\left[o(g)=p^{n}\right]\right\}$, and for $p \in I, T_{p} \neq\left\{0_{T}\right\}$.

Proof: $\quad(1) \Rightarrow(2)$. We use classical proof in [29]. Let $T$ be a countable nontrivial torsion group, i.e., $T \neq\left\{0_{T}\right\}$. Let $o(g)$ be the order of $g$ in $T$. Because $T$ is torsion, $o(g)$ is the least nonzero natural number $n$ such that $n g=0_{T}$. The function $o$ exists in $\mathrm{RCA}_{0}$ ( $o$ is indeed $\Sigma_{0}^{0}$ ).

For every prime $p, T_{p}=\left\{g \in T: \exists n\left[o(g)=p^{n}\right]\right\}$ exists in $\mathrm{RCA}_{0}$. As $g \in T_{p}$ iff $o(g)=p^{n}$ for some $n \leq o(g)$. $T_{p}$ is also $\Sigma_{0}^{0}$.

We now show that any element of $T$ can be uniquely written as a $\mathbb{Z}$-linear sum of elements in $T_{p}$ for various primes $p$.

Let $g \in T$ with order $o(g)$. Factorize $o(g)=q_{1}^{r_{1}} \cdots q_{k}^{r_{k}}$ as prime powers. Let $n_{i}=\frac{o(g)}{q_{i}^{i}}$ for all $i=1, \cdots, k$. Then $n_{i}, 1 \leq i \leq k$, are coprime, so there are integers $a_{i}$ for all $1 \leq i \leq k$ such that $a_{1} n_{1}+\cdots+a_{k} n_{k}=1$. Then,

$$
g=\left(a_{1} n_{1}+\cdots+a_{k} n_{k}\right) g=a_{1} n_{1} g+\cdots+a_{k} n_{k} g .
$$

Because $o\left(n_{i} g\right)=\frac{o(g)}{n_{i}}=q_{i}^{r_{i}}$, each $n_{i} g \in T_{q_{i}}$. Thus, $g \in \sum_{1 \leq i \leq k} T_{q_{i}}$.

We now show that the sum of $g$ is unique. Suppose that there are $x_{i}, y_{i} \in T_{q_{i}}$ such that $g=x_{1}+\cdots+x_{k}=y_{1}+\cdots+y_{k}$. Then $x_{i}-y_{i} \in T_{q_{i}}$ for all $1 \leq i \leq k$, and

$$
x_{1}-y_{1}=\left(y_{2}-x_{2}\right)+\cdots+\left(y_{k}-x_{k}\right) \text {. }
$$

The order of $x_{1}-y_{1}$ is a power of $q_{1}$, but the order of right hand side is a multiplication of powers of $q_{i}$ with $2 \leq i \leq k$. Hence, $x_{1}-y_{1}=0$. Now consider

$$
x_{2}+\cdots+x_{k}=y_{2}+\cdots+y_{k} .
$$

In a similar way, we can show that $x_{2}=y_{2}, \cdots, x_{i}=y_{i}$. Thus, $x_{i}=y_{i}$ for all $i=1, \cdots, k$. This proves the uniqueness of sums.

Hence, $T=\bigoplus_{p \in P} T_{p}$, and we may have $T_{p}=\left\{0_{T}\right\}$ for some primes $p$. 
Now let $I=\left\{p \in P: \exists g \in T-\left\{0_{T}\right\} \exists n\left[o(g)=p^{n}\right]\right\}$. Then $I$ is $\Sigma_{1}^{0}$, so it exists in $\mathrm{ACA}_{0}$. Moreover, $T_{p} \neq\left\{0_{T}\right\} \Leftrightarrow p \in I$. Hence, $I$ is the desired set such that $T=\bigoplus_{p \in I} T_{p}$ with $T_{p} \neq\left\{0_{T}\right\}$ for each $p \in I$.

$(2) \Rightarrow(1)$. Let $f: \mathbb{N} \rightarrow \mathbb{N}$ be a one-to-one function. Let $G$ be an abelian group generated by $\left\{x_{n}: n \in \mathbb{N}\right\}$ with relations $p_{f(m)} x_{m}=0$ for each $m \in \mathbb{N}$. Then $G=\bigoplus_{m \in \mathbb{N}} \mathbb{Z}_{p_{f(m)}} x_{m}$, where for any $n \in \mathbb{N}^{+}, \mathbb{Z}_{n}=\mathbb{Z} / n \mathbb{Z}$. We view elements of $\mathbb{Z}_{n}$ as the least nonnegative representatives under relation $x \sim y \Leftrightarrow x-y \in n \mathbb{Z}=\{n k$ : $k \in \mathbb{Z}\}$, i.e., for $n \geq 1, \mathbb{Z}_{n}=\{i \in \mathbb{N}: i \leq n-1\}$.

Elements of $G$ are of the form $\sum_{i \leq n} a_{i} x_{i}$ for some $a_{i} \in \mathbb{Z}_{p_{f(i)}}$ with each $i \leq n$. So $G$ exists in $\mathrm{RCA}_{0}$. Clearly, $G$ is torsion.

By assumption (2), there is a set $I \subseteq P$ such that $G=\bigoplus_{p \in I} G_{p}$, where for each prime $p, G_{p}=\left\{g \in G: \exists n\left[o(g)=p^{n}\right]\right\}$, and for each $p \in I, G_{p} \neq\left\{0_{G}\right\}$.

By definition of $G$, each element has order a product of $p_{f(m)}$ for various $m$. If $n \notin \operatorname{range}(f), G_{p_{n}}=\left\{0_{G}\right\}$. If $n=f(m)$ for some $m$, then $p_{n} x_{m}=0_{G}$, so $x_{m} \in G_{p_{n}}$, and thus $G_{p_{n}} \neq\left\{0_{G}\right\}$. So

$$
G=\bigoplus_{n \in \text { range }(f)} G_{p_{n}} .
$$

Then $n \in \operatorname{range}(f) \Leftrightarrow p_{n} \in I$. By $\Delta_{1}^{0}$-comprehension, range $(f)$ exists.

We will consider the decomposition theorem of divisible abelian groups. Before proving that, we recall a few reverse mathematical results about countable algebra.

Theorem 6.6 [26] Over $\mathrm{RCA}_{0}$, the following are equivalent:

(1) $\mathrm{ACA}_{0}$

(2) Every abelian group has a torsion subgroup $T$ containing all torsion elements.

(3) Every vector space has a basis.

Definition 6.4 $\left(\mathrm{RCA}_{0}\right)$

(1) For $1 \leq k<\infty, G^{k}$ denotes the direct sum of $k$ many $G$ s, that is, $G^{k}=\bigoplus_{1 \leq i \leq k} G$.

(2) $G^{\infty}$ denotes the direct sum of infinitely many $G$ s, i.e., $G^{\infty}=\bigoplus_{k \in \mathbb{N}} G$. 
Proposition 6.1 $\left(\mathrm{RCA}_{0}\right)$

(1) For $1 \leq k<\infty$, a direct sum $\bigoplus_{1 \leq i \leq k} G_{i}$ of abelian groups is divisible iff for all $1 \leq i \leq k, G_{i}$ is divisible.

(2) A direct sum $\bigoplus_{i \in \mathbb{N}} G_{i}$ of abelian groups is divisible iff for all $i \in \mathbb{N}, G_{i}$ is divisible.

For a prime number $p$, the $p$-quasicyclic group $\mathbb{Z}\left(p^{\infty}\right)=\left\{\frac{n}{p^{m}}: n, m \in \mathbb{Z}\right\} / \mathbb{Z}$ exists in $\mathrm{RCA}_{0}$.

Theorem 6.7 Over $\mathrm{RCA}_{0}$, the following are equivalent:

(1) $\mathrm{ACA}_{0}$

(2) Every divisible abelian group is isomorphic to a direct sum of the form

$$
\bigoplus_{n \in I}\left(\mathbb{Z}\left(p_{n}^{\infty}\right)\right)^{l_{n}} \oplus \bigoplus_{n \in J} \mathbb{Q}
$$

for some $I, J \subseteq \mathbb{N}$, and if $I \neq \varnothing$, then for each $n \in I, 1 \leq l_{n} \leq \infty$.

Proof: $\quad(1) \Rightarrow(2)$. This was already mentioned by Friedman, Simpson and Smith in paper [26], we now provide a complete proof. Let $G$ be a countable divisible abelian group. $\mathrm{ACA}_{0}$ proves the existence of torsion subgroup $T$ of $G$.

We first show that $T$ is a divisible subgroup of $G$. For $g \in T$ and $n \geq 1$, as $G$ is divisible, there is a $g^{\prime} \in G$ such that $g=n g^{\prime}$. Let the order of $g$ be $m$. Then $m n g^{\prime}=0$, so the order of $g^{\prime}$ is also finite. Thus $g^{\prime} \in T$.

By Theorem 6.2, $\mathrm{ACA}_{0}$ proves $T$ is a direct summand of $G$. Let $G=T \oplus F$.

$F$ is a divisible torsion-free subgroup of $G$. For each $x \in F$ and nonzero $n \in \mathbb{Z}$, there is a unique $y \in F$ with $x=n y$ and we write $y=\frac{1}{n} x . F$ is a vector space over the rational field. As $\mathrm{ACA}_{0}$ proves that every vector space has a basis, let $X \subseteq F$ be a basis of $F$. Then $F=\bigoplus_{x \in X} \mathbb{Q} x$, and each $\mathbb{Q} x \cong \mathbb{Q}$ as abelian groups.

Now consider the torsion subgroup $T$. Suppose that $T$ is nontrivial (otherwise, we have done). By Theorem 6.5, $\mathrm{ACA}_{0}$ proves that $T$ is a direct sum of nontrivial primary subgroups. That is, there is some $Z \subseteq P$ such that $T=\bigoplus_{p \in Z} T_{p}$, where 
for each $p \in Z, T_{p}=\left\{g \in T: \exists n\left[o(g)=p^{n}\right]\right\} \neq\left\{0_{G}\right\}$. Since $T$ is divisible, by Proposition 6.1 in this section, each $T_{p}$ is divisible.

Fix $p \in Z$, we now decompose $T_{p}$.

Let $P_{0}=\left\{g \in T_{p}: p g=0\right\} . g \in P_{0}$ iff $g=0$ or $o(g)=p$, where $o(g)$ is the order of $g$ in $T_{p} . P_{0}$ is $\Sigma_{0}^{0}$ with parameter $T_{p}$, and thus exists in $\mathrm{ACA}_{0}$. Another view is that $P_{0}=\left\{g \in T_{p}: p g=0\right\}$ is a $\mathbb{Z}_{p}$-module, where $\mathbb{Z}_{p}$ is the finite field of size $p$. Similarly, $\mathrm{ACA}_{0}$ proves $P_{0}$ has a basis $Y_{0}$ as vector spaces over $\mathbb{Z}_{p}$. So $P_{0}=\bigoplus_{y \in Y_{0}} \mathbb{Z}_{p} y$. List $Y_{0}$ as $y_{0,1}, y_{0,2}, \cdots$ without repetitions. Let $K_{p}=\left\{i \in \mathbb{N}: y_{0, i} \in Y_{0}\right\}$.

For each $y_{0, i} \in Y_{0}$, since $T_{p}$ is divisible, for each $n \geq 1$, there are $y_{n, i} \in T_{p}$ such that $y_{0, i}=p^{n} y_{n, i}$. Let $Y_{n}:=\left\{y_{n, i}: i \in K_{p}\right\}$.

Consider $P_{1}=\left\{g \in T_{p}: o(g)=p^{2}\right\}$. Then $p P_{1}=\left\{p g: g \in P_{1}\right\} \subseteq P_{0}$. We now show that any element in $P_{1}$ is a unique sum of elements in $Y_{0} \cup Y_{1}$. Let $g \in P_{1}$, then $p g \in P_{0}$. There are $y_{0, i_{1}}, \cdots, y_{0, i_{k}} \in Y_{0}, a_{1}, \cdots, a_{k} \in \mathbb{Z}_{p}$ such that

$$
p g=a_{1} y_{0, i_{1}}+\cdots+a_{k} y_{0, i_{k}}
$$

in $P_{0}$ and the expression of $\mathbb{Z}_{p}$-linear sums is unique.

As $y_{0, i_{l}}=p y_{1, i_{l}}$ for each $l=1, \cdots, k$ and $y_{1, i_{l}} \in Y_{1}$, we have

$$
p g=p\left(a_{1} y_{1, i_{1}}+\cdots+a_{k} y_{1, i_{k}}\right)
$$

So $g-\left(a_{1} y_{1, i_{1}}+\cdots+a_{k} y_{1, i_{k}}\right) \in P_{0}$. Then $g-\left(a_{1} y_{1, i_{1}}+\cdots+a_{k} y_{1, i_{k}}\right)$ can be written as a unique $\mathbb{Z}_{p}$-linear combination of elements in $Y_{0}$, and thus, $g$ can be written as a unique $\mathbb{Z}_{p}$-linear combination of elements in $Y_{0} \cup Y_{1}$. So $P_{0} \cup P_{1} \subseteq \bigoplus_{y \in Y_{0} \cup Y_{1}} \mathbb{Z}_{p} y$.

In general, let $P_{n}=\left\{g \in T_{p}: o(g)=p^{n+1}\right\}$. Any element in $P_{n}$ is a unique

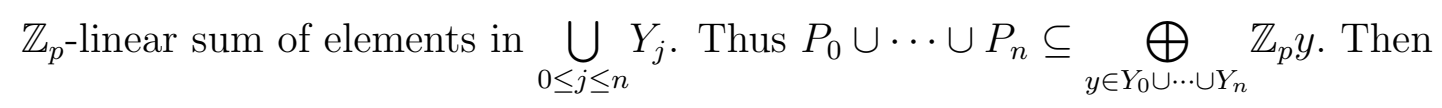

$$
\begin{aligned}
T_{p} \subseteq \bigcup_{n \in \mathbb{N}} P_{n} & \subseteq \bigoplus_{n \in \mathbb{N}} \bigoplus_{y \in Y_{n}} \mathbb{Z}_{p} y \\
& =\bigoplus_{n \in \mathbb{N}} \bigoplus_{i \in K_{p}} \mathbb{Z}_{p} y_{n, i} \subseteq T_{p}
\end{aligned}
$$

Now $T_{p}=\bigoplus_{n \in \mathbb{N}} \bigoplus_{i \in K_{p}} \mathbb{Z}_{p} y_{n, i} \cong \bigoplus_{i \in K_{p}} \bigoplus_{n \in \mathbb{N}} \mathbb{Z}_{p} y_{n, i}$ 
For each $i \in K_{p}$, the subgroup generated by $\left\{y_{n, i}: n \in \mathbb{N}\right\}$ is isomorphic to $\mathbb{Z}\left(p^{\infty}\right)$. Hence, $T_{p} \cong \bigoplus_{i \in K_{p}} \bigoplus_{n \in \mathbb{N}} \mathbb{Z}_{p} y_{n, i} \cong \bigoplus_{i \in K_{p}} \mathbb{Z}\left(p^{\infty}\right)=\left(\mathbb{Z}\left(p^{\infty}\right)\right)^{\left|K_{p}\right|}$, where $\left|K_{p}\right|$ is the size of $K_{p}$ if $K_{p}$ is finite, and $\infty$ otherwise.

This completes the decomposition of $T_{p}$ for a fixed $p \in Z$.

Finally, we have

$$
\begin{aligned}
G \cong T \oplus F & \cong \bigoplus_{p \in Z} T_{p} \oplus F \\
& \cong \bigoplus_{p \in Z}\left(\mathbb{Z}\left(p^{\infty}\right)\right)^{\left|K_{p}\right|} \oplus \bigoplus_{x \in X} \mathbb{Q}
\end{aligned}
$$

Now let $I=\left\{n \in \mathbb{N}: p_{n} \in Z\right\}, J=X$, and for each $n \in I, l_{n}=\left|K_{p_{n}}\right|$. Then $G \cong \bigoplus_{n \in I}\left(\mathbb{Z}\left(p_{n}^{\infty}\right)\right)^{l_{n}} \oplus \bigoplus_{n \in J} \mathbb{Q}$

$(2) \Rightarrow(1)$. Let $f: \mathbb{N} \rightarrow \mathbb{N}$ be a one-to-one function. Consider abelian group $\bigoplus_{m \in \mathbb{N}} \mathbb{Z}\left(p_{f(m)}^{\infty}\right) x_{m}$, denoted by $G$. Elements of $G$ are of the form $\sum_{i \leq n} a_{i} x_{i}$ for some $a_{i} \in \mathbb{Z}\left(p_{f(i)}^{\infty}\right)$ with each $i \leq n$. $G$ exists in $\mathrm{RCA}_{0}$, and is divisible.

By (2), $G$ is a direct sum of subgroups isomorphic to $\mathbb{Q}$ or $\mathbb{Z}\left(p^{\infty}\right)$ for various primes $p$. Let $G \cong \bigoplus_{n \in I}\left(\mathbb{Z}\left(p_{n}^{\infty}\right)\right)^{l_{n}} \oplus \bigoplus_{n \in J} \mathbb{Q}$ for some $I, J \subseteq \mathbb{N}$, and when $I \neq \varnothing$, for each $n \in I, 1 \leq l_{n} \leq \infty$.

Then $I=\operatorname{range}(f), J=\varnothing$, and $l_{n}=1$ for each $n \in I$. Hence, $n \in \operatorname{range}(f)$ iff $n \in I$, so range $(f)$ exists. 


\section{Chapter 7}

\section{Reverse mathematics and modules}

\subsection{Projective modules}

Recall that a $R$-module $P$ is projective if for every surjective homomorphism $\varepsilon: B \rightarrow$ $C$ of $R$-modules and every homomorphism $g: P \rightarrow C$, there exists a homomorphism $f: P \rightarrow B$ such that $g=\varepsilon f$.

\subsubsection{Direct sums of projective modules}

Proposition $7.1\left(\mathrm{RCA}_{0}\right) A$ direct sum $\bigoplus_{0 \leq i \leq n} P_{i}$ is projective iff $P_{i}$ is projective for all $0 \leq i \leq n$.

However, for the infinite case, we have the following theorem.

Theorem 7.1 (1) $\left(\mathrm{RCA}_{0}\right)$ If a direct sum $P=\bigoplus_{i \in \mathbb{N}} P_{i}$ is projective, then $P_{i}$ is projective for all $i \in \mathbb{N}$.

(2) $\left(\Pi_{1}^{1}-\mathrm{CA}_{0}\right)$ If for all $i \in \mathbb{N}, P_{i}$ is projective, then the direct sum $P=\bigoplus_{i \in \mathbb{N}} P_{i}$ is projective.

Proof: We first prove (1) within $\mathrm{RCA}_{0}$. Suppose that $P$ is projective. We will prove that each $P_{i}$ is projective. Given any diagram of the form

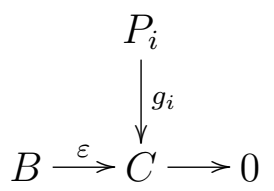


Define $g: P \rightarrow C$ as $g(x)=g_{i}\left(x_{i}\right)$ where $x_{i}$ is the $i$-th column of $x \in P$. As $P$ is projective, there is a $f: P \rightarrow B$ such that $g=\varepsilon f$. For each $z \in P_{i}$, we have

$$
\varepsilon f(\langle 0, \cdots, 0, z, 0, \cdots\rangle)=g(\langle 0, \cdots, 0, z, 0, \cdots\rangle)=g_{i}(z) .
$$

So there is a homomorphism $f_{i}: P_{i} \rightarrow B$ with $f_{i}(z)=f(\langle 0, \cdots, 0, z, 0, \cdots\rangle)$ such that $\varepsilon f_{i}=g_{i}$.

We now prove (2) within $\Pi_{1}^{1}-\mathrm{CA}_{0}$. Suppose that $P_{i}$ is projective for all $i$, and we will prove that $P$ is also projective. Give a surjective homomorphism $\varepsilon: B \rightarrow C$ and a homomorphism $g: P \rightarrow C$, we need to find a homomorphism $f: P \rightarrow B$ such that $\varepsilon f=g$.

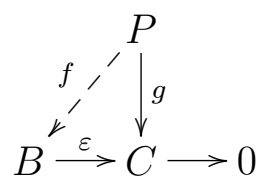

For each $i$, let $g_{i}: P_{i} \rightarrow C$ be a homomorphism with $f_{i}(z)=g(\langle 0, \cdots, 0, z, 0, \cdots\rangle)$. As $P_{i}$ is projective, there is a homomorphism $f_{i}: P_{i} \rightarrow B$ such that

$$
\varepsilon f_{i}(z)=g_{i}(z)=g(\langle 0, \cdots, 0, z, 0, \cdots\rangle)
$$

Then for any $x=\left\langle x_{0}, \cdots, x_{n}\right\rangle \in P$, set $f(x)=f_{0}\left(x_{0}\right)+\cdots+f_{n}\left(x_{n}\right)$, which is in $B$, and we have

$$
\varepsilon f(x)=\sum_{0 \leq i \leq n} \varepsilon f_{i}\left(x_{i}\right)=\sum_{0 \leq i \leq n} g\left(\left\langle 0, \cdots, 0, x_{i}, 0, \cdots\right\rangle\right)=g(x) .
$$

So $f$ exists in $\Pi_{1}^{1}-\mathrm{CA}_{0}$. Note that to define $f$, we use $\left\langle f_{i}: i \in \mathbb{N}\right\rangle$.

The following theorem has already been studied by Yamazaki in [22]. It provides several equivalence characterizations of projective modules, and such equivalence are provable over $\mathrm{RCA}_{0}$. For completeness, we provide a proof here.

Theorem 7.2 (Yamazaki [22]) $\left(\mathrm{RCA}_{0}\right)$ For a $R$-module $P$, the following are equivalent:

(1) $P$ is projective.

(2) If $\varepsilon: B \rightarrow P$ is a surjective homomorphism, then there exists a homomorphism $f: P \rightarrow B$ such that $\varepsilon f=1_{P}$, the identity homomorphism on $P$. 
(3) If $P \cong B / A$, then $P$ is isomorphic to a direct summand of $B$.

(4) $P$ is isomorphic to a direct summand of a free module $F$.

Proof: $\quad(1) \Rightarrow(2)$. As $P$ is projective, there is a homomorphism $f: P \rightarrow B$ such that $\varepsilon f=1_{P}$. That is, the diagram

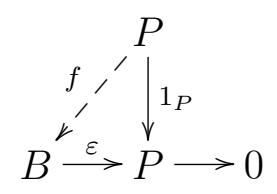

commutes.

$(2) \Rightarrow(3)$. Let $\pi: B \rightarrow B / A$ be the canonical surjective homomorphism, and $g: B / A \rightarrow P$ be an isomorphism. Then $\varepsilon=g \pi$ is a surjective homomorphism from $B$ to $P$. Let $\mu: A \rightarrow B$ be the identical embedding, i.e., for any $x \in A, \mu(x)=x$.

Consider the short exact sequence $0 \rightarrow A \stackrel{\mu}{\rightarrow} B \stackrel{\varepsilon}{\rightarrow} P \rightarrow 0$. By (2), it splits with a splitting $f$. Then by Lemma $1.2, B=A \oplus f(P)$. As $f$ is one-to-one, $P \cong f(P)$, which is a direct summand of $B$.

$(3) \Rightarrow(4)$. By Proposition 1.7, $P \cong F / A$ for some free module $F$. By (3), $P$ is isomorphic to a direct summand of $F$.

$(4) \Rightarrow(1)$. By (4), $P$ is isomorphic to a submodule $Q$ of $F$ via some isomorphism $f$, and $F=Q \oplus A$ for some submodule $A$. As free modules are projective, $F$ is projective, and hence, by Proposition 7.1, $Q$ is projective.

Now we show that $P$ is projective. Give a surjective homomorphism $\varepsilon: B \rightarrow C$ and a homomorphism $g: P \rightarrow C$, we need to find a homomorphism $h: P \rightarrow B$ such that the diagram

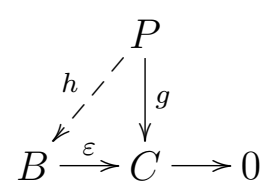

commutes. Consider the homomorphism $g f^{-1}: Q \rightarrow C$. Since $Q$ is projective, there is a homomorphism $h^{\prime}: Q \rightarrow B$ such that $g f^{-1}=\varepsilon h^{\prime}$. Let $h=h^{\prime} f$. Then

$$
\varepsilon h=\varepsilon\left(h^{\prime} f\right)=\left(\varepsilon h^{\prime}\right) f=\left(g f^{-1}\right) f=g\left(f^{-1} f\right)=g .
$$




\subsubsection{Free modules and projective modules over $\Sigma_{1}^{0}$-PIDs}

Free modules are projective, but generally, projective modules may not be free. Now we restrict to $\Sigma_{1}^{0}$-PIDs, because over such special rings, projective modules are always free. Within $\mathrm{RCA}_{0}$, define an integral domain $R$ to be a $\Sigma_{1}^{0}$-PID if every $\Sigma_{1}^{0}$-ideal of $R$ is generated by one element.

Lemma $7.1\left(\mathrm{ACA}_{0}\right)$ Let $R$ be a $\Sigma_{1}^{0}$-PID. A submodule of a free $R$-module is free.

Proof: We reason in $\mathrm{ACA}_{0}$. As we only consider countable modules, we assume that a free $R$-module $F$ is either $\bigoplus_{0 \leq i \leq n} R$ for some $n \in \mathbb{N}$, or $\bigoplus_{i \in \mathbb{N}} R$. We prove it for the case $F=\bigoplus_{i \in \mathbb{N}} R$. The following proof adopts ideas of a classical proof in [30].

Let $N$ be a submodule of $F$. Fix a code for $N$. Now elements of $N$ are coded as natural numbers. For all $i$, let $N_{i}=N \cap \bigoplus_{0 \leq j \leq i} R$, and define a map $f_{i}: N_{i} \rightarrow R$ with $f_{i}\left(\left\langle x_{0}, \cdots, x_{i-1}, x_{i}\right\rangle\right)=x_{i}$. Then $f_{i}$ is a $R$-homomorphism, and $i m\left(f_{i}\right)=N_{i} / N_{i-1}$ is an ideal of $R$. Again, we view elements in $i m\left(f_{i}\right)$ as representatives of equivalence classes under the equivalence relation $x \sim y \Leftrightarrow x-y \in N_{i-1}$ such that their codes are least as natural numbers. $i m\left(f_{i}\right)$ exists in $\mathrm{RCA}_{0}$.

As $R$ is a $\Sigma_{1}^{0}$-PID, for each $i$, there exists a $b_{i}$ such that $\left(b_{i}\right)=i m\left(f_{i}\right)$. Define a function $g$ by setting $g(i)=b_{i}$. Since $g(i)=b_{i}$ if $b_{i} \in i m\left(f_{i}\right)$ and

$$
\forall a \in i m\left(f_{i}\right) \exists c \in R\left[a=c b_{i}\right]
$$

$g$ is $\Pi_{2}^{0}$, thus exists in $\mathrm{ACA}_{0}$.

Let $J=\{j \in \mathbb{N}: g(j) \neq 0\}$. List $J$ as $j_{0}<\cdots j_{n}<j_{n+1}<\cdots$. For each $j_{n} \in J$, define $c_{j_{n}}$ by primitive recursion as follows. Let $c_{j_{0}} \in N_{j_{0}}$ be the element with least code such that $f_{j_{0}}\left(c_{j_{0}}\right)=b_{j_{0}}$. Assume that we have defined $c_{j_{l}}$ for all $l \leq k-1$, to define $c_{j_{k}}$, we let $r_{j_{k}} \in N_{j_{k}}$ be the element with least code such that $f_{j_{k}}\left(r_{j_{k}}\right)=b_{j_{k}}$, and check whether the code of $r_{j_{k}}$ is bigger than or equal to the code of $c_{j_{k-1}}$ or not. If yes, just let $c_{j_{k}}=r_{j_{k}}$; otherwise, we can have $m$ big enough such that the code of $m c_{j_{k-1}}+r_{j_{k}}$ is bigger than the code of $c_{j_{k-1}}$, and we let $c_{j_{k}}=m c_{j_{k-1}}+r_{j_{k}}$. This ensures that the sequence $c_{j_{k}}, k \in \mathbb{N}$, has increasing codes, and we still have

$$
f_{j_{k}}\left(c_{j_{k}}\right)=f_{j_{k}}\left(m c_{j_{k-1}}+r_{j_{k}}\right)=f_{j_{k}}\left(r_{j_{k}}\right)=b_{j_{k}}
$$


$C=\left\{c_{j}: j \in J\right\}$ is $\Delta_{1}^{0}$ in $J$ and hence exists in $\mathrm{ACA}_{0}$.

Claim 1. $C=\left\{c_{j}: j \in J\right\}$ is linearly independent.

Proof of claim 1: For any finite subset $A \subseteq C$, let $A=\left\{c_{i_{1}}, c_{i_{2}}, \cdots, c_{i_{n}}\right\}$ with $i_{1}<i_{2}<\cdots<i_{n}$. If $\sum_{0 \leq k \leq n} d_{i_{k}} c_{i_{k}}=0$ for some $d_{i_{k}} \in R$, then

$$
0=f_{i_{n}}\left(\sum_{0 \leq k \leq n} d_{i_{k}} c_{i_{k}}\right)=\sum_{0 \leq k \leq n} d_{i_{k}} f_{i_{n}}\left(c_{i_{k}}\right)=d_{i_{n}} b_{i_{n}} .
$$

As $b_{i_{n}} \neq 0, d_{i_{n}}=0$, then $\sum_{0 \leq k \leq n-1} d_{i_{k}} c_{i_{k}}=0$. By applying $f_{i_{n-1}}$, we obtain $d_{i_{n-1}}=0$. Continue this process, we arrive at $d_{i_{n}}=d_{i_{n-1}}=\cdots=d_{i_{1}}=0$. So $A$ is linearly independent.

This completes the proof of claim 1.

Claim 2. $C$ generates $N$.

Proof of claim 2: We prove it by contradiction. Assume that there is a least $n \in \mathbb{N}$ such that some $x=\left\langle x_{0}, \cdots, x_{n}\right\rangle \in N_{n}$ that cannot be written as linear combinations of elements of $C$.

By the minimality of $n, x_{n} \neq 0$. Then $f_{n}(x)=x_{n} \in\left(b_{n}\right) \neq\{0\}$, thus, $b_{n} \neq 0$, and $n \in J$. Let $x_{n}=d_{n} b_{n}$ for some $d_{n} \in R-\{0\}$. Then

$$
f_{n}(x)=x_{n}=d_{n} b_{n}=d_{n} f_{n}\left(c_{n}\right)=f_{n}\left(d_{n} c_{n}\right),
$$

so $x-d_{n} c_{n} \in \operatorname{ker}\left(f_{n}\right)=N_{n-1}$.

As $x$ cannot be written as linear combinations of elements of $C$ and $c_{n} \in C$, so does $x-d_{n} c_{n}$. But $x-d_{n} c_{n} \in N_{n-1}$, contradicting the minimality of $n$.

This completes the proof of claim 2.

By claims 1 and 2, $C$ is a basis of $N$, and thus $N$ is free.

Theorem $7.3\left(\mathrm{ACA}_{0}\right)$ Let $R$ be a $\Sigma_{1}^{0}$-PID. A projective $R$-module is free.

Proof: Since $\mathrm{RCA}_{0}$ proves that every projective module is isomorphic to a direct summand of a free module, it proves that every projective module is isomorphic to a submodule of a free module. Let $M$ be a projective $R$-module. There is an isomorphism $f: M \rightarrow M^{\prime}$, where $M^{\prime}$ is a submodule of a free $R$-module. By Lemma 7.1, $\mathrm{ACA}_{0}$ proves $M^{\prime}$ is free. Let $C$ be a basis of $M^{\prime}$. Then $f^{-1}(C):=\left\{f^{-1}(c): c \in\right.$ $C$ \} is a basis of $M$, i.e., $M$ is free. 
Corollary $7.1\left(\mathrm{ACA}_{0}\right)$ Let $R$ be a $\Sigma_{1}^{0}$-PID. A submodule of a projective $R$-module is projective.

\section{$7.2 \quad$ Injective modules}

The notion of injective modules is the dual of projective modules. A $R$-module $I$ is injective if for any monomorphism $\mu: A \rightarrow B$ and any homomorphism $g: A \rightarrow I$, there exists a homomorphism $f: B \rightarrow I$ such that $g=f \mu$.

\subsubsection{Baer's criterion for injective modules}

The following classical lemma is known as Baer's criterion for injective modules.

Lemma 7.2 (Baer's Criterion) A module I over a commutative ring $R$ with identity is injective iff for every ideal $J$ of $R$ and every $R$-module homomorphism $g: J \rightarrow I$, there is a homomorphism $f: R \rightarrow I$ such that $f \uparrow_{J}=g$.

By definition, it is easy to see that $\mathrm{RCA}_{0}$ proves one direction: a module $I$ over a ring $R$ is injective implies that for every ideal $J$ of $R$ and every $R$-module homomorphism $g: J \rightarrow I$, there is a homomorphism $f: R \rightarrow I$ such that $f \uparrow_{J}=g$.

The other direction is equivalent to $\mathrm{ACA}_{0}$, due to Yamazaki in [22]. For completeness, we will provide a proof.

Theorem 7.4 (Yamazaki [22]) The following are equivalent over $\mathrm{RCA}_{0}$.

(1) $\mathrm{ACA}_{0}$

(2) If for every ideal $J$ of $R$ and every $R$-module homomorphism $g: J \rightarrow I$, there is a homomorphism $f: R \rightarrow I$ such that $f \uparrow_{J}=g$, then $I$ is injective.

Proof: $\quad(1) \Rightarrow(2)$. We reason in $\mathrm{ACA}_{0}$. Assume that for an ideal $J$ of $R$, and a $R$-module homomorphism $g: J \rightarrow I$, there is a homomorphism $f: R \rightarrow I$ such that the diagram

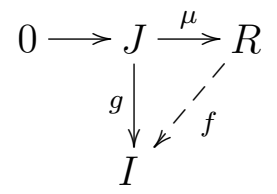


commutes, where $\mu(x)=x$ for $x \in J$. We need to show that for any submodule $A$ of a $R$-module $B$, the identical embedding $\mu: A \rightarrow B$, and a $R$-module homomorphism $g: A \rightarrow I$, there is a homomorphism $f: B \rightarrow I$ making the following diagram commutative.

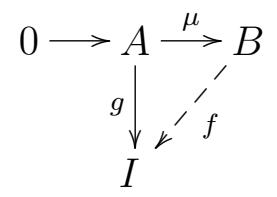

List $B$ as $b_{0}, b_{1}, \cdots$, and let $A_{n}$ be the $R$-module generated by $A \cup\left\{b_{0}, \cdots, b_{n}\right\}$. We extend $g: A \rightarrow I$ to $B$ by stages.

Stage 0. If $A_{0}=A$, then let $f_{0}: A_{0} \rightarrow I$ be the same as $g$. Otherwise, let $S_{0}=\left\{r \in R: r b_{0} \in A\right\}$. Since $A$ is a $R$-module, $S_{0}$ is an ideal of $R$. Define a homomorphism $h_{0}: S_{0} \rightarrow I$ by letting $h_{0}(r)=g\left(r b_{0}\right)$. By assumption, there is a homomorphism $g_{0}: R \rightarrow I$ with $g_{0} \uparrow_{S_{0}}=h_{0}, g_{0}\left(1_{R}\right) \in I$, and for $r \in S_{0}$,

$$
\begin{gathered}
r g_{0}\left(1_{R}\right)=g_{0}(r)=h_{0}(r)=g\left(r b_{0}\right) . \\
0 \longrightarrow S_{0} \longrightarrow R \\
h_{0} \downarrow_{r}^{\prime} g_{0} \\
I
\end{gathered}
$$

So we define $f_{0}: A_{0} \rightarrow I$ by letting $f_{0}\left(b_{0}\right)=g_{0}\left(1_{R}\right)$, and for any $x=a+r b_{0} \in A_{0}$ with $a \in A, r \in R, f_{0}(x)=g(a)+r f_{0}\left(b_{0}\right)$. We claim that $f_{0}$ is a $R$-homomorphism from $A_{0}$ to $I$. Let $x=a+r b_{0}$ and $x^{\prime}=a^{\prime}+r^{\prime} b_{0}$ be two elements in $A_{0}$, and $r^{\prime \prime} \in R$. Then

$$
\begin{aligned}
f_{0}\left(x+x^{\prime}\right) & =g\left(a+a^{\prime}\right)+\left(r+r^{\prime}\right) f_{0}\left(b_{0}\right) \\
& =g(a)+g\left(a^{\prime}\right)+r f_{0}\left(b_{0}\right)+r^{\prime} f_{0}\left(b_{0}\right) \\
& =f_{0}(x)+f_{0}\left(x^{\prime}\right),
\end{aligned}
$$

and

$$
\begin{aligned}
f_{0}\left(r^{\prime \prime} x\right) & =g\left(r^{\prime \prime} a\right)+r^{\prime \prime} r f_{0}\left(b_{0}\right) \\
& =r^{\prime \prime} g(a)+r^{\prime \prime} r f_{0}\left(b_{0}\right) \\
& =r^{\prime \prime} f_{0}(x) .
\end{aligned}
$$


Moreover, $f_{0} \uparrow_{A}=g$. In fact, if $r b_{0} \in A$ with $r \neq 0$, then $r \in S_{0}$, and

$$
f_{0}\left(r b_{0}\right)=r f_{0}\left(b_{0}\right)=r g_{0}\left(1_{R}\right)=g_{0}(r)=h_{0}(r)=g\left(r b_{0}\right) .
$$

Stage $n+1$. Assume that we have $f_{n}: A_{n} \rightarrow I$ extending $f_{n-1}$. If $b_{n+1} \in A_{n}$, do nothing, let $f_{n+1}=f_{n}$. Otherwise, let $S_{n+1}=\left\{r \in R: r b_{n+1} \in A_{n}\right\}$, which is a proper ideal of $R$. Let $h_{n+1}: S_{n+1} \rightarrow I$ by $h_{n+1}(r)=f_{n}\left(r b_{n+1}\right)$. Then there is a $R$-module homomorphism $g_{n+1}: R \rightarrow I$ such that the diagram

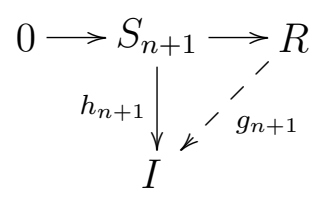

commutes. So for any nonzero $r b_{n+1} \in A_{n}$,

$$
f_{n}\left(r b_{n+1}\right)=h_{n+1}(r)=g_{n+1}(r)=r g_{n+1}\left(1_{R}\right)
$$

Now define $f_{n+1}: A_{n+1} \rightarrow I$ by choosing

$$
f_{n+1}\left(b_{n+1}\right)=g_{n+1}\left(1_{R}\right)
$$

and extending it linearly to all $A_{n+1}$. That is, for $x=a+r b_{n+1} \in A_{n+1}$ with $a \in A_{n}, r \in R$, define

$$
f_{n+1}(x)=f_{n}(a)+r f_{n+1}\left(b_{n+1}\right) .
$$

It is easy to check that $f_{n+1}$ is the homomorphism from $A_{n+1}$ to $I$ with $f_{n+1} \Upsilon_{A_{n}}=f_{n}$.

This provides a mapping from $B$ to $I$ by having $f(x)=f_{n}(x)$ for $x \in A_{n}$. It is obvious that $f$ is well-defined and is a homomorphism, and that $f$ is the limit of $f_{n}$.

$(2) \Rightarrow(1)$. We prove the reversal direction by showing that (2) implies the statement "every divisible abelian group is injective" which is equivalent to $\mathrm{ACA}_{0}$ over $\mathrm{RCA}_{0}$.

Let $D$ be a divisible abelian group, $(n)$ be an ideal of $\mathbb{Z}$, and $g:(n) \rightarrow D$ be a $\mathbb{Z}$-module homomorphism. We will extend $g$ to $\mathbb{Z}$, i.e., define a homomorphism $f: \mathbb{Z} \rightarrow D$ such that

$$
f \Gamma_{(n)}=g .
$$


- If $n=0$, just define $f: \mathbb{Z} \rightarrow D$ to be the zero homomorphism.

- If $n \neq 0, g(n) \in D$. By divisibility of $D$, there is a $c \in D$ such that $g(n)=n c$. Then define a $\mathbb{Z}$-module homomorphism $f: \mathbb{Z} \rightarrow D$ by letting $f(1)=c$, and

$$
f(n)=n f(1)=n c=g(n) .
$$

So for any $x \in(n)$, let $x=m_{x} n$, then

$$
f(x)=m_{x} f(n)=m_{x} g(n)=g\left(m_{x} n\right)=g(x) .
$$

Thus, the assumption of (2) is true for $R=\mathbb{Z}$ and $I=D$. Now by applying (2) to $R=\mathbb{Z}$ and $I=D, D$ is injective.

\subsubsection{Divisible modules and injective modules over $\Sigma_{1}^{0}$-PIDs}

Let $R$ be an integral domain. Recall that a $R$-module $D$ is divisible if for every $d \in D$ and every nonzero $r \in R$, there is a $c \in D$ such that $d=r c$. Classically, a module over a principal ideal domain is injective iff it is divisible. By Proposition 1.11, $\mathrm{RCA}_{0}$ proves that injective modules over an integral domain are divisible. However, to show the other direction, we require $\mathrm{ACA}_{0}$.

Lemma $7.3\left(\mathrm{ACA}_{0}\right)$ Let $R$ be a $\Sigma_{1}^{0}$-PID. A divisible $R$-module $D$ is injective.

Proof: We reason in $\mathrm{ACA}_{0}$. The proof is similar to the one for abelian groups. Let $\mu: A \rightarrow B$ be a given monomorphism. Without loss of generality, we assume that $A$ is a submodule of $B$. For a given homomorphism $g: A \rightarrow D$, we need to find a homomorphism $f: B \rightarrow D$ such that $g=f \mu$.

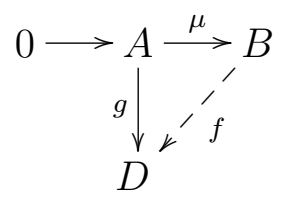

List $B$ as $b_{0}, b_{1}, \cdots$, and let $A_{n}$ be the submodule of $B$ generated by $A \cup$ $\left\{b_{0}, \cdots, b_{n}\right\}$ for $n \in \mathbb{N}$. $A_{n}$ exists by $\Sigma_{1}^{0}$-comprehension. We will extend $g$ to $B$ by stages. 
Stage 0. If $b_{0} \in A$, then $A=A_{0}$, let $g_{0}=g$ with domain $A_{0}$. Otherwise, consider set

$$
S_{0}=\left\{r \in R: r b_{0} \in A\right\}
$$

As $A$ is a $R$-module, $S_{0}$ is an ideal of $R$. Obviously, $S_{0}$ is $\Delta_{1}^{0}$, so by assumption that $R$ is a $\Sigma_{1}^{0}$-PID, there is a $t_{0} \in R$ such that $S_{0}=\left(t_{0}\right)$. If $t_{0}=0$, just choose $g_{0}\left(b_{0}\right)$ as 0 . If $t_{0} \neq 0, g\left(t_{0} b_{0}\right) \in D$; by divisibility of $D$, there is a $c_{0} \in D$ such that $g\left(t_{0} b_{0}\right)=t_{0} c_{0}$, and define

$$
g_{0}\left(b_{0}\right)=c_{0}
$$

Then for any $x \in A_{0}, x=a+r b_{0}$ for some $a \in A$ and $r \in R$, set

$$
g_{0}(x)=g(a)+r g_{0}\left(b_{0}\right)
$$

We can check $g_{0}$ is a $R$-module homomorphism from $A_{0}$ to $D$, as in Theorem 7.4. We now show that $g_{0}$ extends $g$. If $r b_{0} \in A$ with $r \neq 0$, then $g_{0}\left(r b_{0}\right)=r c_{0}$. As $r \in S_{0}$, there is a $r^{\prime} \in R$ such that $r=r^{\prime} t_{0}$. Then

$$
g_{0}\left(r b_{0}\right)=r c_{0}=r^{\prime} t_{0} c_{0}=r^{\prime} g\left(t_{0} b_{0}\right)=g\left(r^{\prime} t_{0} b_{0}\right)=g\left(r b_{0}\right)
$$

Stage $n+1$. Assume that we have already defined $g_{n}: A_{n} \rightarrow D$ extending $g_{n-1}$. Now consider $b_{n+1}$.

If $b_{n+1} \in A_{n}$, then $A_{n+1}=A_{n}$ and we let $g_{n+1}=g_{n}$. Otherwise, let

$$
S_{n+1}=\left\{r \in R: r b_{n+1} \in A_{n}\right\} .
$$

Again, $S_{n+1}$ is an ideal of $R$, and $S_{n+1}$ is $\Sigma_{1}$. By assumption that $R$ is a $\Sigma_{1}^{0}$-PID, $S_{n+1}$ is a PID and we let $S_{n+1}=\left(t_{n+1}\right)$. If $t_{n+1}=0$, define $g_{n+1}\left(b_{n+1}\right)=0$. If $t_{n+1} \neq 0$, as $t_{n+1} b_{n+1} \in A_{n}$, and hence $g_{n}\left(t_{n+1} b_{n+1}\right) \in D$. As $D$ is divisible, there is a $c_{n+1} \in D$ such that

$$
g_{n}\left(t_{n+1} b_{n+1}\right)=t_{n+1} c_{n+1} .
$$

We now define $g_{n+1}\left(b_{n+1}\right)=c_{n+1}$, and extend it linearly to $A_{n+1}$ by letting

$$
g_{n+1}\left(a+r b_{n+1}\right)=g_{n}(a)+r g_{n+1}\left(b_{n+1}\right)
$$

for $a \in A_{n}$ and $r \in R$. We can check that $g_{n+1}$ is a $R$-module homomorphism with domain $A_{n+1}$ and that $g_{n+1} \Upsilon_{A_{n}}=g_{n}$. 
Then $B=\bigcup_{n \in \mathbb{N}} A_{n}$, and for all $n, g_{n+1}$ extends $g_{n} . f: B \rightarrow D$ defined by

$$
f(x)=g_{n}(x)
$$

for some $n$ with $x \in A_{n}$ is well-defined and is a $R$-module homomorphism extending g. $f$ is the desired homomorphism.

In [26], it was proved that the statement "every divisible abelian group is injective" is equivalent to $\mathrm{ACA}_{0}$ over $\mathrm{RCA}_{0}$. Together with Lemma 7.3, we obtain:

Theorem 7.5 The following are equivalent over $\mathrm{RCA}_{0}$ :

(1) $\mathrm{ACA}_{0}$

(2) Every divisible module over a $\Sigma_{1}^{0}$-PID is injective.

(3) Every divisible abelian group is injective.

\section{Proposition $7.2\left(\mathrm{RCA}_{0}\right)$}

(1) Every quotient of a divisible module over a $\Sigma_{1}^{0}$-PID is divisible.

(2) Every quotient of an injective module over a $\Sigma_{1}^{0}$-PID is divisible.

Proof: (1). Let $D$ be a divisible $R$-module, and $\varepsilon: D \rightarrow B$ a surjective homomorphism. We show that $B$ is divisible. For any nonzero $b \in B$, find a $d \in D$ with $\varepsilon(d)=b$. By divisibility of $D$, for any nonzero $r \in R$, there is a $d^{\prime} \in D$ such that $d=r d^{\prime}$. Then $b=\varepsilon(d)=r \varepsilon\left(d^{\prime}\right)$ with $\varepsilon\left(d^{\prime}\right) \in B$.

(2). Let $M$ be an injective module over a $\Sigma_{1}^{0}$-PID. $\mathrm{RCA}_{0}$ proves that $M$ is divisible and that every quotient of a divisible module over a $\Sigma_{1}^{0}$-PID is divisible. Hence, $\mathrm{RCA}_{0}$ proves that every quotient of $M$ is divisible.

By Lemma 7.3, $\mathrm{ACA}_{0}$ proves that every divisible module over a $\Sigma_{1}^{0}$-PID is injective, and we obtain the following corollary.

Corollary $7.2\left(\mathrm{ACA}_{0}\right)$ Every quotient of an injective module over a $\Sigma_{1}^{0}$-PID is injective. 


\section{References}

[1] B. Anderson and B. Csima, "A bounded jump for the bounded Turing degrees," Notre Dame J. Formal Logic, vol. 55, pp. 245-264, 2014.

[2] B. Anderson, B. Csima, and K. lange, "Bounded low and high sets," Arch. Math. Logic, vol. 56, pp. 507-521, 2017.

[3] A. M. Kach, K. Lange, and R. Solomon, "Degrees of orders on torsion-free abelian groups," Ann. Pure Appl. Logic, vol. 164, pp. 822-836, 2013.

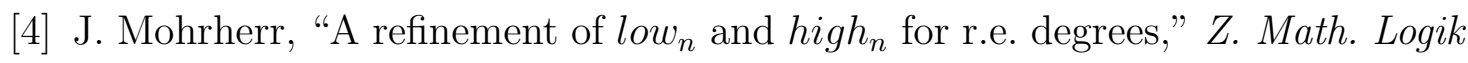
Grundlag. Math., vol. 32, pp. 5-12, 1986.

[5] S. B. Cooper, Degrees of Unsolvability. PhD thesis, Leicester University, 1971.

[6] S. B. Cooper, Computability Theory. Chapman and Hall/CRC, 2004.

[7] R. W. Robinson, "Interpolation and embedding in the recursively enumerable degrees," Annals of Mathematics, vol. 93, pp. 285-314, 1971.

[8] S. B. Cooper, "Minimal pairs and high recursively enumerable degrees," $J$. Symbolic Logic, vol. 39, pp. 655-660, 1974.

[9] D. Miller, The Relationship between the Structure and the Degrees of Recursively Enumerable Sets. PhD thesis, University of Chicago, Chicago, Illinois, 1981.

[10] R. A. Shore and T. A. Slaman, "Working below a high recursively enumerable degree," J. Symbolic Logic, vol. 58, pp. 824-859, 1993. 
[11] Ambos-Spies, Jockusch, Shore, and Soare, "An algebraic decomposition of the recursively enumerable degrees and the coincidence of several degree classes with the promptly degrees," Trans. Amer. Math. Soc., vol. 281, pp. 109-128, 1984.

[12] M. Bickford and C. Mills, "Lowness properties of r.e. sets," Typewritten unpublished manuscript, 1982.

[13] J. C. G. Jockusch and R. I. Soare, " $\Pi_{1}^{0}$ classes and degrees of theories," Trans. Amer. Math. Soc., vol. 173, pp. 33-56, 1972.

[14] R. Solomon, " $\Pi_{1}^{0}$ classes and ordered groups," Ann. Pure Appl. Logic, vol. 115, pp. 279-302, 2002.

[15] G. Metakides and A. Nerode, "Effective content of field theory," Ann. Pure Appl. Logic, vol. 17, pp. 289-320, 1979.

[16] C. J. Martin, Computability Theory and Ordered Groups. PhD thesis, University of Connecticut, Storrs, CT, 2015.

[17] F. Stephan, "Martin-löf random and PA-complete sets," Logic Colloquium, 2002, Proceedings of the Annual European Summer Meeting of the Association for Symbolic Logic and the Colloquium Logicum, held in Münster, Germany, August 3C11, 2002. Lecture Notes in Logic, vol. 27, pp. 342-348, 2006.

[18] P. Odifreddi, Classical Recursion Theory. Volumes I and II, North HollandElsevier, Amsterdam, New York, Oxford, Tokyo, 1989 and 1999.

[19] K. Hatzikiriakou and S. G. Simpson, "WKL $\mathrm{WL}_{0}$ and orderings of countable abelian groups," Contemporary Mathematics, vol. 106, pp. 177-180, 1990.

[20] S. G. Simpon, Subsystems of Second Order Arithmetic. Springer-Verlag, 1999.

[21] R. Solomon, "Reverse mathematics and fully ordered groups," Notre Dame J. Formal Logic, vol. 39, pp. 157-189, 1998. 
[22] T. Yamazaki, "Reverse mathematics and commutative ring theory (slides)," Conference on "Computability Theory and Foundations of Mathematics", Tokyo Institute Of Technology, February 18-20, 2013.

[23] R. G. Downey, S. Lempp, and J. R. Mileti, "Ideals in computable rings," J. Algebra, vol. 314, pp. 872-887, 2007.

[24] T. Sato, Reverse Mathematics and Countable Algebraic Systems. PhD thesis, Tohoku University, Sendai, Japan, 2016.

[25] S. G. Simpson, "Ordinal numbers and the Hilbert basis theorem," J. Symbolic Logic, vol. 53, pp. 961-974, 1988.

[26] H. M. Friedman, S. G. Simpson, and R. L. Smith, "Countable algebra and set existence axioms," Ann. Pure Appl. Logic, vol. 25, pp. 141-181, 1983.

[27] A. Nies, Computability and Randomness. Oxford University Press, Inc. New York, 2009.

[28] J. C. G. Jockusch and R. A. Shore, "Pseudo-jump operators. I: the r. e. case," Trans. Amer. Math. Soc., vol. 275, pp. 599-609, 1983.

[29] I. Kaplansky, Infinite Abelian Groups. University of Michigan, Michigan, 1954.

[30] R. J. Hilton and U. Stammbach, A Course in Homological Algebra. Second edition, Graduate Texts in Mathematics, 4. Springer-Verlag, New York, 1997. 